\title{
Field exercise testing in COPD
}

Citation for published version (APA):

Andrianopoulos, V. (2017). Field exercise testing in COPD. [Doctoral Thesis, Maastricht University]. Datawyse / Universitaire Pers Maastricht. https://doi.org/10.26481/dis.20170510va

Document status and date:

Published: 01/01/2017

DOI:

10.26481/dis.20170510va

Document Version:

Publisher's PDF, also known as Version of record

\section{Please check the document version of this publication:}

- A submitted manuscript is the version of the article upon submission and before peer-review. There can be important differences between the submitted version and the official published version of record.

People interested in the research are advised to contact the author for the final version of the publication, or visit the DOI to the publisher's website.

- The final author version and the galley proof are versions of the publication after peer review.

- The final published version features the final layout of the paper including the volume, issue and page numbers.

Link to publication

\footnotetext{
General rights rights.

- You may freely distribute the URL identifying the publication in the public portal. please follow below link for the End User Agreement:

www.umlib.nl/taverne-license

Take down policy

If you believe that this document breaches copyright please contact us at:

repository@maastrichtuniversity.nl

providing details and we will investigate your claim.
}

Copyright and moral rights for the publications made accessible in the public portal are retained by the authors and/or other copyright owners and it is a condition of accessing publications that users recognise and abide by the legal requirements associated with these

- Users may download and print one copy of any publication from the public portal for the purpose of private study or research.

- You may not further distribute the material or use it for any profit-making activity or commercial gain

If the publication is distributed under the terms of Article $25 \mathrm{fa}$ of the Dutch Copyright Act, indicated by the "Taverne" license above, 


\section{FIELD EXERCISE TESTING IN COPD}

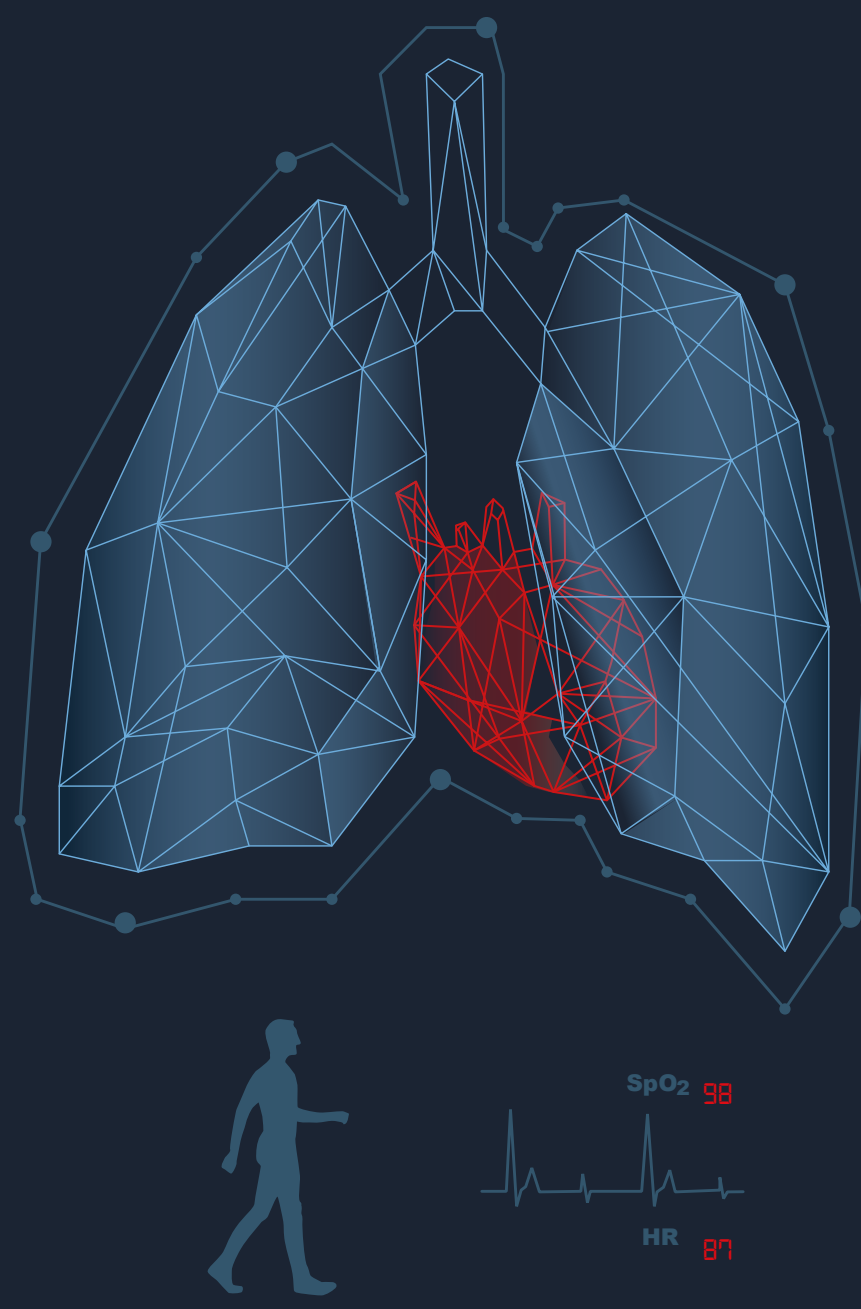

Vasileios Andrianopoulos 
Copyright @ Vasileios Andrianopoulos, Maastricht 2017

ISBN: 9789461596871

Lay out: Datawyse

Cover design: Lina Koulountzou

Production: Datawyse | Universitaire Pers Maastricht

The thesis was financially supported by $\mathrm{ClRO}+$, center of expertise for chronic organ failure, the Netherlands

Printing and distribution of this thesis was financially supported by $\mathrm{CIRO}+$, center of expertise for chronic organ failure, the Netherlands 


\title{
Field exercise testing in COPD
}

\author{
DISSERTATION \\ to obtain the degree of Doctor at Maastricht University, \\ on the authority of the Rector Magnificus, Prof. dr. Rianne M. Letschert \\ in accordance with the decision of the Board of Deans, \\ to be defended in public \\ on Wednesday, 10 May 2017, at 10:00 am
}

by

Vasileios Andrianopoulos 


\section{Supervisors}

Prof. dr. M.A. Spruit

Prof. dr. E.F.M. Wouters

\section{Co-supervisor}

Prof. dr. I. Vogiatzis, National and Kapodistrian University of Athens, Greece

\section{Members of the Degree Committee}

Prof. dr. G.J. Wesseling (Chairman)

Prof. dr. H.P. Brunner-La Rocca

Prof. dr. Y.F. Heijdra, Radboud University Medical Center, Nijmegen, the Netherlands Prof. dr. J. Kooman

Prof. dr. N.G. Koulouris, National and Kapodistrian University of Athens, Greece 


\section{Table of contents}

Chapter 1 General Introduction

Chapter 2 Characteristics and determinants of endurance cycle ergometry and six-minute walk distance in patients with COPD

Chapter 3 Six-minute walk distance in patients with chronic obstructive pulmonary disease: Which reference equations should we use?

Chapter 4 Prognostic value of variables derived from the six-minute walk test in patients with COPD: Results from the ECLIPSE study

Chapter 5 Determinants of exercise-induced oxygen desaturation including pulmonary emphysema in COPD: Results from the ECLIPSE study

Chapter 6 Exercise-induced oxygen desaturation in COPD patients without resting hypoxemia

Chapter 7 Transcutaneous carbon-dioxide partial pressure trends during sixminute walk test in patients with very severe COPD

Chapter 8 General Discussion

Summary

Valorization

Acknowledgements

Curriculum vitae

List of publications 

Chapter

General Introduction 



\section{CHRONIC OBSTRUCTIVE PULMONARY DISEASE}

Chronic obstructive pulmonary disease (COPD) is a common preventable and treatable disease which is characterized by persistent respiratory symptoms and airflow limitation that is due to airway and/or alveolar abnormalities usually caused by significant exposure to noxious particles or gases. ${ }^{1}$ It includes two key components: the chronic bronchitis which is partially reversible, and pulmonary emphysema (parenchymal destruction) that is an irreversible component. ${ }^{1}$ COPD may be asymptomatic in its early stages but cause severe symptoms such as dyspnea, cough and sputum overproduction in later stages. In the progression of the disease, a decline in forced expiratory volume in 1 second $\left(\mathrm{FEV}_{1}\right)$ occurs due to loss of lung elasticity and increase in airways resistance in small and/or large airways. In some patients with advanced disease severity, reduced $\mathrm{FEV}_{1}$ is accompanied by arterial hypoxemia with or without hypercapnia indicating respiratory insufficiency. ${ }^{2}$ Regarding the prevalence of COPD in Europe, a number of well-designed studies showed prevalence estimates ranging from $4 \%$ to $10 \%$ among the adults population while new research suggests as many as 66 million people in Europe live with COPD., ${ }^{3,4}$ Statistics for global population have shown that $4.8 \%$ of people in the entire world are diagnosed with COPD. ${ }^{5}$ These findings may only partly represent the burden of the disease because a significant proportion of patients is undiagnosed or misdiagnosed. $3,6,7$

Besides the high prevalence of COPD, the multi-morbidity of disease represents a real public health problem and a major burden on health-care resources. ${ }^{8,9}$ According to the Global Initiative for Chronic Obstructive Lung Disease (GOLD) report of 2017, COPD is a leading cause of morbidity and mortality worldwide and social burden is both substantial and increasing. ${ }^{1}$ Despite worldwide medical research, health care effort and costs, mortality in COPD reveal a continuing upward trend, in contrast with other major causes of death such as cancer and cardiovascular disease. Globally more than 3 million people died of COPD in 2012 and this was the $6 \%$ of all deaths globally. ${ }^{10}$ The World Health Organization (WHO) estimates that COPD will be the third most common worldwide cause of death and disability by 2030, from its current fourth ranking. ${ }^{11}$

The most important risk factor for COPD in developed countries is the tobacco smoke, with $85 \%$ of all cases attributable to smoking ${ }^{8,12}$, while in developing world the exposure to noxious particles and gases (air pollution) often contributes to the prevalence of COPD. ${ }^{1,13}$ Although tobacco smoking is a significant risk factor increasing the odds for COPD development by 5 times $^{12}$, fewer than $50 \%$ cigarette smokers develop clinically significant COPD during their lifetime. ${ }^{14,15}$ Passive smoking may also contribute to respiratory symptoms and COPD due to inhaled particles and gases. ${ }^{16}$ Besides exposures, host factors predispose individuals to develop COPD. These factors include genetic disorders, abnormal lung development and accelerated aging. ${ }^{1}$ In some rare cases, which represent the $1-5 \%$ of people with COPD, the disease results from a 
genetic abnormality that causes low levels of a protein called alpha-1 antitrypsin or from recurrent respiratory infections in childhood. ${ }^{17,18}$ In symptomatic individuals, the diagnosis of COPD is based on spirometry confirming $\mathrm{FEV}_{1}<80 \%$ and a ratio of $\mathrm{FEV}_{1}$ and forced vital capacity (FEV 1 /FVC) below 0.70 after bronchodilation. ${ }^{19}$ Until recently, COPD classification was largely based on $\mathrm{FEV}_{1}$, however, updated GOLD report comprises four quadrants (A-B-C-D) based on symptoms and risk of exacerbations, besides airflow limitation, for classifying patients with COPD. ${ }^{20}$

\section{IMPACT ON EXERCISE CAPACITY AND PHYSICAL ACTIVITY}

Exercise capacity defined as the maximum amount of sustainable physical exertion ${ }^{21}$, and physical activity described as the quantity of body movements produced by skeletal muscles $^{22}$ are significantly reduced in patients with COPD compared to healthy individuals. ${ }^{23,24}$ Markedly impaired exercise capacity and physical activity in COPD is usually reduced in proportion to disease severity as assessed by the GOLD staging system. ${ }^{25,26}$ Patients with COPD often complain for dyspnea that limits the duration and intensity of exercise activities or tasks that can be sustainable. There appears to be an important role for ventilatory limitations which become more prominent as disease progresses and often correlates well with the intensity of dyspnea. ${ }^{27}$

Exercise capacity in COPD may be negatively affected by several factors related to respiratory system such as ventilatory constrains, hyperinflation, diminished oxygen uptake, alveolar wall destruction with impaired gas exchange, increased respiratory muscle fatigue, and decreased ventilatory reserve. ${ }^{28-32}$ Dynamic ventilatory constraints during exercise deteriorated over time can also significantly contribute to exercise capacity deterioration. ${ }^{33}$ Moreover, yet even factors that are not directly related to the lungs may have a negative impact on exercise capacity in COPD including early lactate production $^{34-37}$, muscle dysfunction ${ }^{38,39}$, and cardiovascular deconditioning ${ }^{40,41}$ usually compounded by sedentary lifestyle ${ }^{42,43}$. Consequently, all of these factors results in different grades of dyspnea and leg-fatigue that can be occurred even in relatively low levels of physical activity or exercise intensity in patients with COPD. Indeed, a fair correlation of exercise capacity and physical activity has been already reported. ${ }^{44}$ In COPD, a vicious circle may occur as dyspnea is associated with abstention from physical activity that leads to muscle decondition which in turn results in increased ventilatory requirements causing even higher dyspnea and decrements in exercise capacity. ${ }^{45-47}$ Patients with COPD can be trapped in the vicious cycle of declining physical activity levels and increasing symptoms with exercise (Figure 1). 


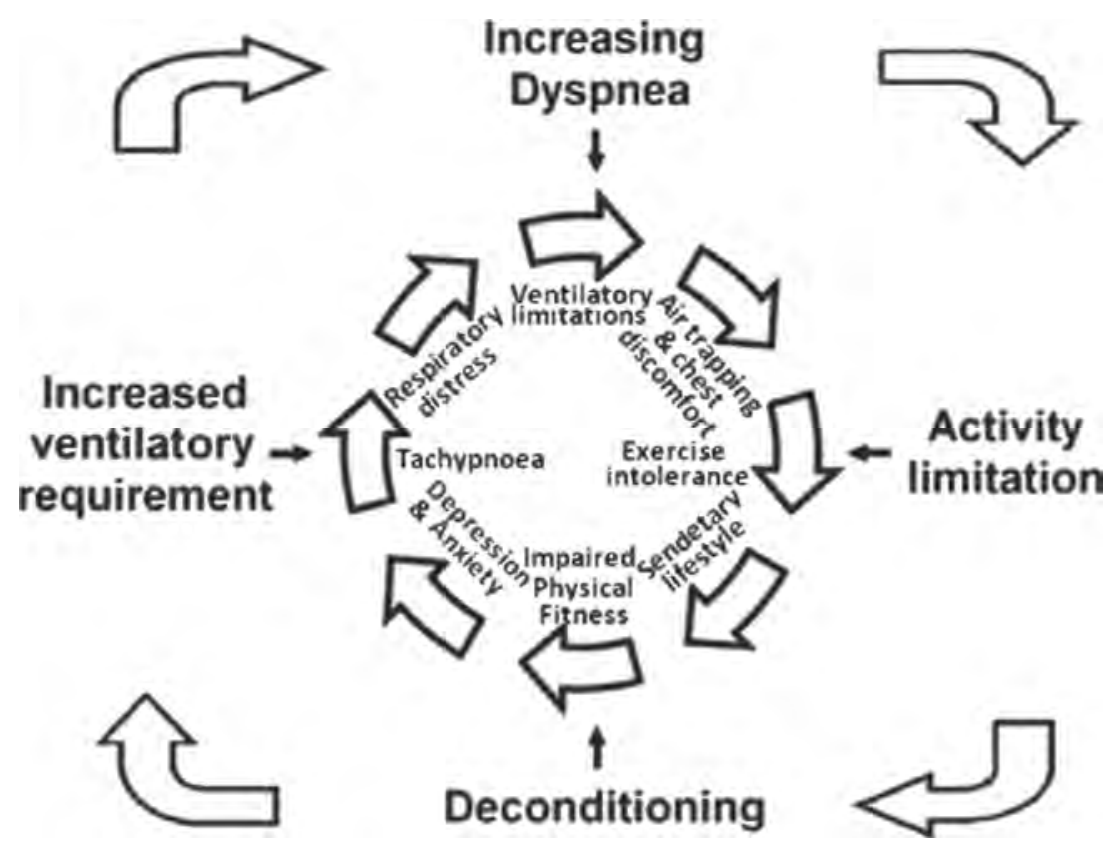

Figure 1. The vicious circle of inactivity in COPD can result in low exercise capacity and inactivity

\section{EVALUATION OF EXERCISE CAPACITY IN COPD}

The evaluation of exercise capacity can be considered as important as the evaluation of pulmonary function in COPD. Evidence has shown that exercise capacity is even superior to single functional measurements obtained at rest (e.g., forced expiratory volume in 1 second) in demonstrating the positive effect of a specific intervention. ${ }^{48}$ It provides useful information for the impact of disease severity in patients' functional status, the effectiveness of therapeutic interventions and it has also prognostic value. ${ }^{48}$

Exercise capacity can be assessed by maximal and submaximal tests, which reflect different pathophysiological properties. Several exercise tests have been developed for measuring exercise capacity and can be categorized in two types: A) the field-based walk tests such as the six-minute walk test and the shuttle walk test, and B) the laboratory tests such as the incremental and cycle endurance ergometer tests. ${ }^{49}$ These tests have different primary outcomes (i.e. walking distance, cycle endurance time, oxygen consumption etc.) that may reflect different physiological parameters. Maximal exercise capacity is commonly assessed by incremental cycle ergometry, whereas the six-minute walk test (6MWT) and cycle endurance test (CET) are responsive to interventions such as drug therapy or exercise training. ${ }^{50-52}$ Specifically, in the evaluation of exercise capacity, simple improvements in exercise tolerance test 


\section{Chapter 1}

performance, submaximal or maximum aerobic capacity levels, and the functional state between respiratory patients can be observed. The evaluation of exercise capacity can help to demonstrate to patients how they are progressing in relation to their exercise capacity and to detect changes in performance over time or after therapeutic interventions. $^{53}$

\section{FIELD WALKING TESTS}

Measurement of walking distance was first advocated by Balke in 1963 as a means of assessing physical fitness. ${ }^{54}$ Nowadays, field walking tests are widely used and play a key role in the evaluation of functional exercise capacity in COPD. ${ }^{55-57}$ They usually include the shuttle walk test (SWT), which is implemented as incremental (ISWT) or as endurance shuttle walk test (ESWT), and the six-minute walk test (6MWT). ${ }^{55-58}$ Field walking tests can be considered as great clinical instruments for assessing prognosis, determining outcome of clinical trials and evaluating response to treatment. Both, SWT and 6MWT employ walking task and do not require expensive and sophisticated technology. ${ }^{55,56}$

The available data have indicated that both 6MWT and SWT are valid, reliable and responsive for the assessment of exercise capacity in COPD. The choice of an individual test may depend on the circumstances and purpose of the test. ${ }^{55}$ The importance of field walking test is reflected by the fact that exercise capacity constitutes an important outcome as a major determinant of impaired health status and as a predicting factor of hospitalization and mortality in COPD. ${ }^{59}$ Moreover, exercise capacity has become an integral part of the management of patients with COPD. ${ }^{60}$ There is also a substantial body of evidence that supports the conceptualisation of the 6MWT as a test of functional exercise capacity in patients with COPD while new insights further strengthen its clinical value.

\section{SIX-MINUTE WALK TEST AND ITS CLINICAL VALUE}

The six-minute walk test (6MWT) was first described by Guyatt and colleagues as an effective, simple and safe tool for assessing walking capacity in clinical practice. ${ }^{61}$ The $6 \mathrm{MWT}$ is self-paced test and is considered to be a test of submaximal exercise capacity, even though some patients reach maximal exercise levels. ${ }^{62,63}$ It is performed by instructing the participant to walk as far as possible in six-minute along a flat corridor and it is well-tolerated as walking is the most common pattern of activity in daily life. ${ }^{55}$ Side-effects of chest pain, dyspnea, or musculoskeletal pain are usually mild to moderate, while serious adverse events have not been described due to the self-paced 
nature of the test. ${ }^{48,55,56,64}$ The main outcome of the $6 \mathrm{MWT}$ is the six-minute walk distance (6MWD), which is measured by actual meters or feet and expressed as percentage of predicted value. In addition, oxygen saturation $\left(\mathrm{SpO}_{2}\right)$ and heart rate (HR) are also recorded during walking by the use of pulse oxymetry. Perceived levels of dyspnea and subjective leg-fatigue are measured the beginning and the end of the 6MWT using validated measurement scales, such as the Borg scale. Standardised instructions and encouragement are commonly given during the walking test. ${ }^{55,56}$

The 6MWT is widely used to measure functional exercise capacity, assess prognosis and evaluate response to treatment across a wide range of chronic respiratory diseases. It has been proven as a reliable clinical instrument in providing reproducible data which are used to detect and quantify changes in exercise capacity after an intervention in COPD. ${ }^{65-67}$ Evidence suggests that the minimal clinically important difference (MCID) for the 6MWT is conservatively estimated between 25 and 33 meters (median estimate $30 \mathrm{~m}) .{ }^{67}$ Practically, 6MWT outcomes regarding patients' health status alterations can be used to prompt a change in medical interventions and management of COPD. In some cases, 6MWD can be used to estimate peak oxygen uptake. ${ }^{68}$ Moreover, exercise intensity can be prescribed from the 6MWT using typically the $80 \%$ of maximum walking speed throughout the test, however, this is not particularly accurate because of the speed which can be variable during walking. In case that a patient walked 300 meters the maximum walk speed can be estimated by the equation of [300 x $10 \div 1000$ $=3.0 \mathrm{~km} /$ hour $].^{69}$ Given the ubiquitous clinical use of the $6 \mathrm{MWT}$, clinicians should be familiar with $6 \mathrm{MWT}$ procedures, limitations and test utility. ${ }^{70,71}$

\section{SIX-MINUTE WALK TEST AND DETECTION OF EXERCISE-INDUCED DESATURATION}

Six-minute walk test due to the simplicity of performance and the high responsiveness to arterial oxygen desaturation is the preferred exercise test to screen patients for exercise-induced desaturation (EID) in COPD. ${ }^{55,56}$ It is documented that 6MWT is more sensitive to detect EID compared to cycle ergometry testing. ${ }^{72}$ There is no uniform definition of EID, however, decline of oxygen saturation ( $\mathrm{SpO}_{2}$ nadir) to $88 \%$ or lower at exertion is commonly accepted for the discrimination of patients with and without EID. $^{73-75}$

EID is highly prevalent among patients with COPD and especially in those with more advance disease severity. In COPD patients with moderate to severe lung disease (mean $\mathrm{FEV}_{1} 43 \%$ pred.), the prevalence of exercise-induced desaturation on $6 \mathrm{MWT}$ was $62 \%$, where desaturation was defined as a drop of $>4 \%$ in $\mathrm{SpO}_{2}$ to $<90 \%$. ${ }^{76}$ The presence of EID can be associated with reduced daily physical activity, poor exercise performance, a faster decline in $\mathrm{FEV}_{1}$, and worse prognosis which support its clinical importance. ${ }^{31,76,77}$ 


\section{Chapter 1}

Several determinants of oxygen desaturation can be used to predict which COPD patients will or won't exhibit EID during the $6 \mathrm{MWT}^{78}{ }^{78}$ These determinants include $\mathrm{FEV}_{1}$, diffusion capacity for carbon monoxide (DLCO), baseline standard pulse oxymetry $\left(\mathrm{SpO}_{2}\right)$ or a combination of thereof. ${ }^{79-83}$ Furthermore, there are evidences that EID is related to emphysema $^{84,85}$ and to increased pulmonary arterial pressure ${ }^{86}$. EID may also be triggered by depressed ventilatory drive which may be typically accompanied by exercise-induced hypercapnia $\left(\mathrm{pCO}_{2}>46 \mathrm{mmHg}\right)$ and low $\mathrm{pH}^{87}$

\section{SIX-MINUTE WALK TEST AND PROGNOSIS}

The association of the 6MWD with hospitalization and mortality is well documented. ${ }^{55}$, 77, 88 Lower 6MWD has been associated with increased hospitalization and mortality in patients with COPD. Particularly, patients with 6MWD lower of $357 \mathrm{~m}$ have increased risk of hospitalization, whereas a 6MWD threshold of $334 \mathrm{~m}$ is a strong predictor of mortality in COPD. ${ }^{89}$ A 6MWD reduction of $30 \mathrm{~m}$ or more is associated with increased risk for mortality but not hospitalization due to exacerbation in patients with COPD. ${ }^{90}$ Moreover, 6MWD of $325 \mathrm{~m}$ or lower has been demonstrated as a threshold for predicting postoperative pulmonary complications with $77 \%$ sensitivity and $100 \%$ specificity, with similar strong predictive value with $\mathrm{FEV}_{1} \cdot{ }^{91}$ Besides 6MWD, walking speed, walk-work, distance-saturation products, and exercise-induced desaturation during 6MWT have demonstrated a long-term prognostic value. ${ }^{92-95}$

\section{AIMS OF THE THESIS}

Clinical exercise testing is a complex concept regarding to exercise pathophysiology in patients with COPD and the interpretation of exercise outcomes. Therefore, the main objectives of this thesis are:

1. To investigate the characteristics of functional exercise performance and determinants of the CET and 6MWT in a large clinical cohort of COPD patients.

2. To explore the impact of several 6MWD reference equations for adults in patients with chronic obstructive pulmonary disease (COPD) and factors accountable for different 6MWD\% predicted values.

3. To study the association between 6MWT-derived variables and mortality as well as hospitalization in COPD patients and to compare it with the BODE index.

4. To investigate the relationship between EID and patient-related outcomes during $6 \mathrm{MWT}$, including pulmonary emphysema in a large well-characterized cohort of COPD subjects. 
5. To validate a proposed cut-off of baseline- $\mathrm{SpO}_{2} \leq 95 \%$ as simple screening procedure to predict EID during 6MWT and study the prevalence and characteristics of patients exhibited EID to $\mathrm{SpO}_{2}$ nadir $\leq 88 \%$.

6. To study $\mathrm{pCO}_{2}$ response during the $6 \mathrm{MWT}$ and compare clinical characteristics between COPD patients with and without $\mathrm{pCO}_{2}$ retention and those with and without EIH during the 6MWT.

\section{OUTLINE OF THE THESIS}

Chapter 1 provides a background of exercise pathophysiology in COPD and the 6MWT as a widely-used exercise assessment in COPD

Chapter 2 provides more insights in the characteristics and determinants of endurance cycle ergometry and six-minute walk distance in patients with COPD.

Chapter 3 presents impact of several 6MWD reference equations on 6MWD predicted values of patients with COPD and the factors accountable for the discrepancy in reference values.

Chapter 4 evaluates the prognostic value of the 6MWT-derived variables to predict 3-year mortality and hospitalization.

Chapter 5 describes the role of emphysema in EID and the determinants of EID in emphysematous patients during the 6MWT.

Chapter 6 reports for the accuracy of certain cut-off of baseline-SpO $\mathrm{S}_{2}(\leq 95 \%)$ as simple screening procedure to predict EID and demonstrates determinants of EID during the 6MWT.

Chapter 7 describes the exercise-induced $\mathrm{pCO}_{2}$ response and demonstrates determinants of $\mathrm{pCO}_{2}$ retention and exercise-induced hypercapnia $(\mathrm{EIH})$ during the 6MWT.

Chapter 8 provides a general discussion regarding the findings of this thesis and their clinical implications. 


\section{REFERENCES}

1. Global Initiative for Chronic Obstructive Lung Disease (GOLD). Global strategy for diagnosis, management, and prevention of chronic obstructive pulmonary disease. Revised 2017.

2. Pauwels RA, Buist AS, Calverley PM, Jenkins CR, Hurd SS and Committee GS. Global strategy for the diagnosis, management, and prevention of chronic obstructive pulmonary disease. NHLBI/WHO Global Initiative for Chronic Obstructive Lung Disease (GOLD) Workshop summary. Am J Respir Crit Care Med. 2001; 163: 1256-76.

3. Halbert RJ, Isonaka S, George D and Iqbal A. Interpreting COPD prevalence estimates: what is the true burden of disease? Chest. 2003; 123: 1684-92.

4. Adeloye D, Chua S, Lee C, et al. Global and regional estimates of COPD prevalence: Systematic review and meta-analysis. J Glob Health. 2015; 5: 020415.

5. Vos T, Flaxman AD, Naghavi M, et al. Years lived with disability (YLDs) for 1160 sequelae of 289 diseases and injuries 1990-2010: a systematic analysis for the Global Burden of Disease Study 2010. Lancet. 2012; 380: 2163-96.

6. Pauwels RA and Rabe KF. Burden and clinical features of chronic obstructive pulmonary disease (COPD). Lancet. 2004; 364: 613-20.

7. Halbert RJ, Natoli JL, Gano A, Badamgarav E, Buist AS and Mannino DM. Global burden of COPD: systematic review and meta-analysis. Eur Respir J. 2006; 28: 523-32.

8. ATS. Standards for the diagnosis and care of patients with chronic obstructive pulmonary disease. American Thoracic Society. Am J Respir Crit Care Med. 1995; 152: S77-121.

9. Lopez AD and Murray CC. The global burden of disease, 1990-2020. Nat Med. 1998; 4: 1241-3.

10. World Health Organization Chronic obstructive pulmonary disease (COPD), Fact sheet $\mathrm{N}^{\circ} 315$.

11. World Health Organization Chronic obstructive pulmonary disease (COPD). WHO, 2011. http://www.who.int/respiratory/copd

12. Raherison C and Girodet PO. Epidemiology of COPD. Eur Respir Rev. 2009; 18: 213-21.

13. Chen J, Schooling C, Johnston J, Hedley A and McGhee S. How does socioeconomic development affect COPD mortality? An age-period-cohort analysis from a recently transitioned population in China. PLoS ONE. 2011; 6: e24348.

14. Barnes PJ. Chronic obstructive pulmonary disease. N Engl J Med. 2000; 343: 269-80.

15. Rennard SI and Vestbo J. COPD: the dangerous underestimate of 15\%. Lancet. 2006; 367: 1216-9.

16. Yin $\mathrm{P}$, Jiang $\mathrm{CQ}$, Cheng KK, et al. Passive smoking exposure and risk of COPD among adults in China: the Guangzhou Biobank Cohort Study. Lancet. 2007; 370: 751-7.

17. Marciniuk DD, Hernandez P, Balter M, et al. Alpha-1 antitrypsin deficiency targeted testing and augmentation therapy: a Canadian Thoracic Society clinical practice guideline. Can Respir J. 2012; 19 : 109-16.

18. Silverman EK and Speizer FE. Risk factors for the development of chronic obstructive pulmonary disease. Med Clin North Am. 1996; 80: 501-22.

19. Chronic obstructive pulmonary disease in over 16s: diagnosis and management (NICE CG101), 2010 https://www.nice.org.uk/guidance/cg101/resources/chronic-obstructive-pulmonary-disease-in-over16s-diagnosis-and-management-3510932393158.

20. Vestbo J, Hurd SS, Agusti AG, et al. Global Strategy for the Diagnosis, Management, and Prevention of Chronic Obstructive Pulmonary Disease: GOLD Executive Summary. Am J Respir Crit Care Med. 2013; 187: 347-65.

21. Goldstein R. Clinical Methods: The History, Physical, and Laboratory Examinations. 3rd edition. 1990.

22. Caspersen CJ, Powell KE and Christenson GM. Physical activity, exercise, and physical fitness: definitions and distinctions for health-related research. Public health reports. 1985; 100: 126-31.

23. Vorrink SN, Kort HS, Troosters T and Lammers JW. Level of daily physical activity in individuals with COPD compared with healthy controls. Respir Res. 2011; 12: 33. 
24. Johnson-Warrington V, Harrison S, Mitchell K, Steiner M, Morgan M and Singh S. Exercise capacity and physical activity in patients with COPD and healthy subjects classified as Medical Research Council dyspnea scale grade 2. J Cardiopulm Rehabil Prev. 2014; 34: 150-4.

25. Pinto-Plata VM, Celli-Cruz RA, Vassaux C, et al. Differences in cardiopulmonary exercise test results by American Thoracic Society/European Respiratory Society-Global Initiative for Chronic Obstructive Lung Disease stage categories and gender. Chest. 2007; 132: 1204-11.

26. Andersson M, Stridsman C, Roenmark E, Lindberg A and Emtner M. Physical activity and fatigue in chroni obstructive pulmonary disease - A population based study. Respir Med. 2015; 109: 1048-57.

27. Lahaije AJ, van Helvoort HA, Dekhuijzen PN and Heijdra YF. Physiologic limitations during daily life activities in COPD patients. Respir Med. 2010; 104: 1152-9.

28. Aliverti A and Macklem PT. Last Word on Point:Counterpoint: The major limitation to exercise performance in COPD is 1 ) inadequate energy supply to the respiratory and locomotor muscles, 2) lower limb muscle dysfunction, 3) dynamic hyperinflation. J Appl Physiol. 2008; 105: 763.

29. Debigare R and Maltais F. Last Word on Point:Counterpoint: The major limitation to exercise performance in COPD is 1) inadequate energy supply to the respiratory and locomotor muscles, 2) lower limb muscle dysfunction, 3) dynamic hyperinflation. J Appl Physiol. 2008; 105: 764.

30. O'Donnell D and Webb K. Last Word on Point:Counterpoint: The major limitation to exercise performance in COPD is 1) inadequate energy supply to the respiratory and locomotor muscles, 2) lower limb muscle dysfunction, 3) dynamic hyperinflation. J Appl Physiol. 2008; 105: 765.

31. Vogiatzis I, Zakynthinos $G$ and Andrianopoulos V. Mechanisms of physical activity limitation in chronic lung diseases. Pulm Med. 2012; 2012: 634761.

32. O'Donnell DE. Ventilatory limitations in chronic obstructive pulmonary disease. Med Sci Sports Exerc. 2001; 33: S647-55.

33. Oga T, Nishimura K, Tsukino M, Sato S, Hajiro T and Mishima M. Exercise capacity deterioration in patients with COPD: longitudinal evaluation over 5 years. Chest. 2005; 128: 62-9.

34. Maltais F, Simard AA, Simard C, Jobin J, Desgagnes P and LeBlanc P. Oxidative capacity of the skeletal muscle and lactic acid kinetics during exercise in normal subjects and in patients with COPD. Am J Respir Crit Care Med. 1996; 153: 288-93.

35. Ichiwata T, Sasao G, Abe T, et al. Oxidative capacity of the skeletal muscle and lactic acid kinetics during exercise in healthy subjects and patients with COPD. Adv Exp Med Biol. 2010; 662: 537-43.

36. Casaburi R, Patessio A, Ioli F, Zanaboni S, Donner CF and Wasserman K. Reductions in exercise lactic acidosis and ventilation as a result of exercise training in patients with obstructive lung disease. Am Rev Respir Dis. 1991; 143: 9-18.

37. Engelen MP, Schols AM, Does JD, Gosker HR, Deutz NE and Wouters EF. Exercise-induced lactate increase in relation to muscle substrates in patients with chronic obstructive pulmonary disease. Am J Respir Crit Care Med. 2000; 162: 1697-704.

38. Jobin J, Maltais F, Doyon JF, et al. Chronic obstructive pulmonary disease: capillarity and fiber-type characteristics of skeletal muscle. J Cardiopulm Rehabil. 1998; 18: 432-7.

39. Rabinovich RA and Vilaro J. Structural and functional changes of peripheral muscles in chronic obstructive pulmonary disease patients. Curr Opin Pulm Med. 2010; 16: 123-33.

40. Maclntyre NR. Mechanisms of functional loss in patients with chronic lung disease. Respir Care. 2008; 53: 1177-84.

41. Casaburi R. Impacting patient-centred outcomes in COPD: deconditioning. Eur Respir Rev 2006; 15: 42-6.

42. Altenburg WA, de Greef $\mathrm{MH}$, ten Hacken $\mathrm{NH}$ and Wempe JB. A better response in exercise capacity after pulmonary rehabilitation in more severe COPD patients. Respir Med. 2012; 106: 694-700.

43. Agusti A. Systemic effects of chronic obstructive pulmonary disease: what we know and what we don't know (but should). Proc Am Thorac Soc. 2007; 4: 522-5.

44. Zwerink M, van der Palen J, van der Valk P, Brusse-Keizer M and Effing T. Relationship between daily physical activity and exercise capacity in patients with COPD. Respir Med. 2013; 107: 242-8.

45. Pitta F. Physical Activities in Daily Life in Patients with COPD: Characterization, Impact of Acute Exacerbations and Pulmonary Rehabilitation. Leuven University Press, 2005. 


\section{Chapter 1}

46. Polkey $\mathrm{Ml}$ and Moxham J. Attacking the disease spiral in chronic obstructive pulmonary disease: an update. Clin Med. 2011; 11: 461-4.

47. Troosters $T$, van der Molen $T$, Polkey $M$, et al. Improving physical activity in COPD: towards a new paradigm. Respir Res. 2013; 14: 115.

48. Palange $\mathrm{P}$, Ward $\mathrm{SA}$, Carlsen $\mathrm{KH}$, et al. Recommendations on the use of exercise testing in clinical practice. Eur Respir J. 2007; 29: 185-209.

49. Fotheringham I, Meakin G, Punekar YS, Riley JH, Cockle SM and Singh SJ. Comparison of laboratory- and field-based exercise tests for COPD: a systematic review. Int J Chron Obstruct Pulmon Dis. 2015; 10: 62543.

50. Pepin V, Brodeur J, Lacasse $Y$, et al. Six-minute walking versus shuttle walking: responsiveness to bronchodilation in chronic obstructive pulmonary disease. Thorax. 2007; 62: 291-8.

51. Laviolette L, Bourbeau J, Bernard S, et al. Assessing the impact of pulmonary rehabilitation on functional status in COPD. Thorax. 2008; 63: 115-21.

52. Borel B, Provencher S, Saey D and Maltais F. Responsiveness of Various Exercise-Testing Protocols to Therapeutic Interventions in COPD. Pulm Med. 2013; 2013: 410748.

53. Vilaró J, Resqueti $V$ and Fregonezi G. Clinical assessment of exercise capacity in patients with chronic obstructive pulmonary disease. Rev Bras Fisioter. 2008; 12: 249-59.

54. Balke B. A Simple Field Test for the Assessment of Physical Fitness. [Report] Civ Aeromed Res Inst. 1963: 1-8.

55. Singh SJ, Puhan MA, Andrianopoulos V, et al. An official systematic review of the European Respiratory Society/American Thoracic Society: measurement properties of field walking tests in chronic respiratory disease. Eur Respir J. 2014; 44: 1447-78.

56. Holland AE, Spruit MA, Troosters T, et al. An official European Respiratory Society/American Thoracic Society technical standard: field walking tests in chronic respiratory disease. Eur Respir J. 2014; 44: 142846.

57. Garvey C, Boylan AM, Miller DL, et al. Field walking tests in chronic respiratory disease. Ann Am Thorac Soc. 2015; 12: 446-7.

58. Brown CD and Wise RA. Field tests of exercise in COPD: the six-minute walk test and the shuttle walk test. COPD. 2007; 4: 217-23.

59. Oga T, Nishimura K, Tsukino M, Sato S and Hajiro T. Analysis of the factors related to mortality in chronic obstructive pulmonary disease: role of exercise capacity and health status. Am J Respir Crit Care Med. 2003; 167: 544-9.

60. Spruit MA and Wouters EF. New modalities of pulmonary rehabilitation in patients with chronic obstructive pulmonary disease. Sports Med. 2007; 37: 501-18.

61. Guyatt GH, Sullivan MJ, Thompson PJ, et al. The 6-minute walk: a new measure of exercise capacity in patients with chronic heart failure. Can Med Assoc J. 1985; 132: 919-23.

62. Casas A, Vilaro J, Rabinovich R, et al. Encouraged 6-min walking test indicates maximum sustainable exercise in COPD patients. Chest. 2005; 128: 55-61.

63. Holland AE, Dowman L, Fiore J, Jr., Brazzale D, Hill CJ and McDonald CF. Cardiorespiratory responses to 6-minute walk test in interstitial lung disease: not always a submaximal test. BMC Pulm Med. 2014; 14: 136.

64. Clini EM and Crisafulli E. Exercise capacity as a pulmonary rehabilitation outcome. Respiration. 2009; 77: 121-8.

65. Solway S, Brooks D, Lacasse $Y$ and Thomas S. A qualitative systematic overview of the measurement properties of functional walk tests used in the cardiorespiratory domain. Chest. 2001; 119: 256-70.

66. Bellet RN, Adams L and Morris NR. The 6-minute walk test in outpatient cardiac rehabilitation: validity, reliability and responsiveness--a systematic review. Physiotherapy. 2012; 98: 277-86.

67. McCarthy B, Casey D, Devane D, Murphy K, Murphy E and Lacasse Y. Pulmonary rehabilitation for chronic obstructive pulmonary disease. Cochrane DB Syst Rev. 2015; 2: CD003793.

68. Ross RM, Murthy JN, Wollak ID and Jackson AS. The six minute walk test accurately estimates mean peak oxygen uptake. BMC Pulm Med. 2010; 10: 31. 
69. Houchen-Wolloff L, Latimer $L$ and Steiner M. Aerobic exercise testing. In: Scott A and Gidlow C, (eds.). Clinical exercise science. New York: Routledge, 2016, p. 56-7.

70. Smith DR. Use of the 6-min walk test: A pro and con review. Am Coll Chest Physicians 2009; 23:1

71. Holland A, Spruit MA and Singh S. How to carry out a field walking test in chronic respiratory disease. Breathe. 2015; 11: 128-39.

72. Poulain M, Durand F, Palomba B, et al. 6-minute walk testing is more sensitive than maximal incremental cycle testing for detecting oxygen desaturation in patients with COPD. Chest. 2003; 123: 1401-7.

73. Panos RJ and Eschenbacher W. Exertional desaturation in patients with chronic obstructive pulmonary disease. COPD. 2009; 6: 478-87.

74. Garvey C, Tiep B, Carter R, Barnett M, Hart M and Casaburi R. Severe exercise-induced hypoxemia. Respir Care. 2012; 57: 1154-60.

75. Roberts MM, Cho JG, Sandoz JS and Wheatley JR. Oxygen desaturation and adverse events during 6-min walk testing in patients with COPD. Respirology. 2015; 20: 419-25.

76. van Gestel AJ, Clarenbach CF, Stowhas AC, et al. Prevalence and prediction of exercise-induced oxygen desaturation in patients with chronic obstructive pulmonary disease. Respiration. 2012; 84: 353-9.

77. Casanova C, Cote C, Marin JM, et al. Distance and oxygen desaturation during the 6-min walk test as predictors of long-term mortality in patients with COPD. Chest. 2008; 134: 746-52.

78. Hadeli KO, Siegel EM, Sherrill DL, Beck KC and Enright PL. Predictors of oxygen desaturation during submaximal exercise in 8,000 patients. Chest. 2001; 120: 88-92.

79. Owens GR, Rogers RM, Pennock BE and Levin D. The diffusing capacity as a predictor of arterial oxygen desaturation during exercise in patients with chronic obstructive pulmonary disease. N Eng/ J Med. 1984; 310: 1218-21.

80. Kelley MA, Panettieri RA, Jr. and Krupinski AV. Resting single-breath diffusing capacity as a screening test for exercise-induced hypoxemia. Am J Med. 1986; 80: 807-12.

81. Knower MT, Dunagan DP, Adair NE and Chin R, Jr. Baseline oxygen saturation predicts exercise desaturation below prescription threshold in patients with chronic obstructive pulmonary disease. Arch Intern Med. 2001; 161: 732-6.

82. Hussein K, Farouk Alkarn A, Hamdi S, El-Sokkary R and Shams H. Factors predicting exercise-induced oxygen desaturation in stable COPD. Eur Respir J. 2012; 40.

83. Gupta SS, Gothi D, Narula G and Sircar J. Correlation of BMI and oxygen saturation in stable COPD in Northern India. Lung India. 2014; 31: 29-34.

84. Taguchi O, Gabazza EC, Yoshida M, et al. CT scores of emphysema and oxygen desaturation during lowgrade exercise in patients with emphysema. Acta Radiol. 2000; 41: 196-7.

85. Kent BD, Mitchell PD and McNicholas WT. Hypoxemia in patients with COPD: cause, effects, and disease progression. Int J Chron Obstruct Pulmon Dis. 2011; 6: 199-208.

86. Christensen CC, Ryg MS, Edvardsen A and O.H. S. Relationship between exercise desaturation and pulmonary haemodynamics in COPD patients. Eur Respir J. 2005; 25: 1126-7.

87. O'Donnell DE, D'Arsigny C, Fitzpatrick $M$ and Webb KA. Exercise hypercapnia in advanced chronic obstructive pulmonary disease: the role of lung hyperinflation. Am J Respir Crit Care Med. 2002; 166: 663-8.

88. Spruit MA, Polkey MI, Celli B, et al. Predicting outcomes from 6-minute walk distance in chronic obstructive pulmonary disease. J Am Med Dir Assoc. 2012; 13: 291-7.

89. Spruit MA, Watkins ML, Edwards LD, et al. Determinants of poor 6-min walking distance in patients with COPD: the ECLIPSE cohort. Respir Med. 2010; 104: 849-57.

90. Polkey MI, Spruit MA, Edwards LD, et al. Six-minute-walk test in chronic obstructive pulmonary disease: minimal clinically important difference for death or hospitalization. Am J Respir Crit Care Med. 2013; 187: 382-6.

91. Keeratichananont W, Thanadetsuntorn C and Keeratichananont S. Value of preoperative 6-minute walk test for predicting postoperative pulmonary complications. Ther Adv Respir Dis. 2016; 10: 18-25.

92. Carter R, Holiday DB, Nwasuruba C, Stocks J, Grothues C and Tiep B. 6-minute walk work for assessment of functional capacity in patients with COPD. Chest. 2003; 123: 1408-15. 


\section{Chapter 1}

93. Lettieri CJ, Nathan SD, Browning RF, Barnett SD, Ahmad S and Shorr AF. The distance-saturation product predicts mortality in idiopathic pulmonary fibrosis. Respir Med. 2006; 100: 1734-41.

94. Studenski S, Perera S, Patel K, et al. Gait speed and survival in older adults. JAMA. 2011; 305: 50-8.

95. Golpe R, Perez-de-Llano LA, Mendez-Marote L and Veres-Racamonde A. Prognostic value of walk distance, work, oxygen saturation, and dyspnea during 6-minute walk test in COPD patients. Respir Care. 2013; 58: 1329-34. 


\section{Chapter}

\section{Characteristics and determinants of endurance cycle ergometry and six-minute walk distance in patients with COPD}

Vasileios Andrianopoulos, Scott S. Wagers, Miriam T.J. Groenen, Lowie E.G.W. Vanfleteren, Frits M.E. Franssen, Frank W.J.M. Smeenk, Ioannis Vogiatzis, Emiel F.M. Wouters, Martijn A. Spruit and On behalf of the $\mathrm{CIRO}+$ Rehabilitation Network 


\section{Chapter 2}

\section{ABSTRACT}

\section{Background}

Exercise tolerance can be assessed by the cycle endurance test (CET) and six-minute walk test (6MWT) in patients with Chronic Obstructive Pulmonary Disease (COPD). We sought to investigate the characteristics of functional exercise performance and determinants of the CET and 6MWT in a large clinical cohort of COPD patients.

\section{Methods}

A dataset of 2053 COPD patients (43\% female, age: $66.9 \pm 9.5$ years, FEV 1 : 48.2 $\pm 23.2 \%$ predicted) was analyzed retrospectively. Patients underwent, amongst others, respiratory function evaluation; medical tests and questionnaires, one maximal incremental cycle test where peak work rate was determined and two functional exercise tests: a CET at $75 \%$ of peak work rate and 6MWT. A stepwise multiple linear regression was used to assess determinants.

\section{Results}

On average, patients had impaired exercise tolerance (peak work rate: $56 \pm 27 \%$ pred., $6 \mathrm{MWT}$ : $69 \pm 17 \%$ pred.). A total of 2002 patients had CET time of duration (CET-T end less than $20 \mathrm{~min}$, while only 51 (2.5\%) of the patients achieved 20min of CET-T end. In former patients, the percent of predicted peak work rate achieved differed significantly between men ( $48 \pm 21 \%$ predicted) and women ( $67 \pm 31 \%$ predicted). In contrast, CET-T end $_{\text {. }}$ was longer in men $(286 \pm 174$ s vs $250 \pm 153 s, p<0.001)$. Also, six minute walking distance (6MWD) was higher in men compared to women, both in absolute terms as in percent of predicted (443m, 67\%pred. vs 431m, 72\%predicted, $\mathrm{p}<0.05)$. Gender was associated with the CET-T end but BMI, FEV 1 and FRC were related to the $6 \mathrm{MWD}$ highlighting the different determinants of exercise performance between CET and 6MWT.

\section{Conclusions}

CET- $_{\text {end }}$ is a valuable outcome of CET as it is related to multiple clinical aspects of disease severity in COPD. Gender difference should temper the interpretation of CET. 


\section{INTRODUCTION}

Constant work rate cycle ergometry, the so-called cycle endurance test (CET), has been put forth as an important assessment of exercise tolerance for patients with chronic obstructive pulmonary disease (COPD).$^{1-3}$ CET endurance time (CET- $T_{\text {end }}$ ) can be used in the evaluation of exercise tolerance. ${ }^{4}$ Yet our understanding of CET lags behind that of the commonly used six minute walk test $(6 \mathrm{MWT}) .^{5-8}$ In fact, the first report of minimal clinically important difference for the CET has only been published in $2008 .^{9}$

Neder and colleagues ${ }^{10}$, in a detailed study aimed at plotting power duration relationship from a series CETs, found that the average critical power asymptote (i.e.: the power at which an individual can cycle for 20 minutes) in patients with COPD was $81 \%$ of the peak work rate achieved on a maximal incremental test. Anecdotal clinical experience, however, suggests that few patients with COPD can cycle that long, even at $75 \%$ of peak work rate, that is the level at which most CETs are performed. ${ }^{11}$ CET performed at this fixed proportion of peak incremental workload capacity does not cause identical physiological stress among patients. ${ }^{12}$ Furthermore, 20 minutes is considered the maximal allowable CET-T end for a $\mathrm{CET}^{13}$, and the test is usually stopped at that point.

Constant work rate endurance protocols in CET are based on the maintenance of exercise effort until the exhaustion. ${ }^{14}$ Although CET is considered as sub-maximal exercise, it is aimed to assess the maximal aerobic capacity of patients and it is suggested as a highly repeatable test across run-in visits. ${ }^{15}$ However, the utility of CETs at $75 \%$ peak work rate comes into doubt as more patients would cycle to the obligatory stopping point of 20 minutes. ${ }^{10}$ Similarly, the 6MWT even though self-paced, it can be considered as a maximal sustainable exercise in patients with COPD. ${ }^{16}$ Both testing modalities for assessing exercise tolerance may provoke different physiological responses and cause different levels of dyspnea and fatigue during the exertion to patients with COPD. ${ }^{17,18}$ Furthermore, the sex of patients has been related to dyspnea in the $6 \mathrm{MWT}^{19}$ and may also determine the exertion during the CET. From a physiological point of view, CET and 6MWT may not interchangeable exercise tests and the determinants of exercise performance between the tests can differ.

Herein, we report a retrospective analysis of $\mathrm{CET}_{-} \mathrm{T}_{\text {end }}$ in a large clinical cohort of patients with COPD undergoing initial evaluation for pulmonary rehabilitation (PR). ${ }^{20}$ We sought to compare the results of CETs in a large clinical cohort to those of a 6MWT and we sought to determine how often a clinical cohort of patients with COPD achieved a maximal CET-T end 20 minutes during a CET performed at a predetermined $75 \%$ of peak work rate. Moreover, we investigated the determinants of CET- $T_{\text {end }}$ and $6 M W D$. 


\section{METHODS}

\section{Design and participants}

We extracted data from the records of 2991 patients with the diagnosis of COPD who were evaluated during the initial assessment of a comprehensive pulmonary rehabilitation program at $\mathrm{ClRO}+$, center of expertise for chronic organ failure in Horn (The Netherlands) between January 1, 2005 and July 1, 2013. ${ }^{20}$ Of these records, 2053 patients met the following inclusion criteria: all necessary data present, a primary diagnosis of COPD, a $F E V_{1} / F V C$ ratio of $\leq 0.70$, evidence that they had reached a physiological maximum during the incremental maximal cardiopulmonary exercise test on a stationary cycle ergometer (i.e., ventilation $\geq 85 \%$ of maximal voluntary ventilation; heart rate $\geq 80 \%$ of the age-predicted heart rate; symptom score for dyspnea $\geq 7 / 10$ points; and/or symptoms score for fatigue $\geq 7 / 10$ points), CET done at $75 \%$ of peak work rate, and not representing a repeat admission for the same patient. Ethical approval was not indicated because all of the tests were done as part of the routine initial assessment, and analyzed retrospectively. The Board of Directors of $\mathrm{CIRO}+$ approved the use of de-identified patients' record.

As part of routine initial assessment, patients underwent, amongst other tests and questionnaires, a maximal incremental cycle test where peak work rate is determined. Subsequently, on a different day, patients performed a CET at 75\% of the determined peak work rate. Patients also performed two $6 \mathrm{MWTs}{ }^{21}$ while the test with the longest distance was used for further analysis. All tests were done according to ATS/ERS guidelines. ${ }^{1,8}$ Routine post-bronchodilator spirometry, physical examination, medical history, and Medical Research Council (MRC) dyspnea scale were obtained. Using dual energy $x$-ray absorptiometry (DEXA), the fat free mass index (FFMI) for each individual was calculated.

\section{Statistical analysis}

All analyses were carried out using SPSS 19, Graphpad Prism 6 and Sigmaplot 12. Data are reported as means with standard deviation $( \pm S D)$. For parametric data, comparison between men and women were made using a two tailed unpaired t-test, and for categorical data Pearson Chi-Square tests were used, as appropriate. Frequency distributions were used to determine performance quartiles for the CET-T end and sixminute walk distance (6MWD) allowing for comparison of the clinical parameters between quartiles. For all comparisons, patients reaching CET-T end 20 minutes (51 patients, $2.5 \%$ ) were excluded from the dataset of 2053 patients as they represented an artificial stopping point, and thereby could artificially reduce standard error. After excluding this group of patients, a dataset of 2002 patients was analyzed for detecting significant differences. For parametric data quartiles were compared using one way 
ANOVA and for non-parametric data quartiles were compared using Kruskal-Wallis test. All multiple comparisons were followed by Bonferonni correction. A stepwise multiple linear regression was used to determine clinical parameters that independently influenced test performance. The level of significance was set at $p<0.05$.

\section{RESULTS}

\section{Clinical parameters}

On average, patients ( $\mathrm{n}=2002 ; \mathrm{CET}_{\text {end }}<20 \mathrm{~min}$ ) had moderate to very severe COPD, a normal BMI, normal resting arterial oxygen and carbon dioxide tension, and symptoms of dyspnea. Gender differences were noticed: men were older, and had a significantly higher height, weight, BMI, FFMI, and $\mathrm{FEV}_{1}$ \%predicted compared to women. Then again, women had a significantly worse DLCO. One out of five patients was using longterm oxygen therapy (LTOT) (Table 1).

Table 1. Demographics of COPD patients

\begin{tabular}{|c|c|c|c|}
\hline Characteristics & $\begin{array}{l}\text { All patients } \\
(n=2002)\end{array}$ & $\begin{array}{l}\text { Males } \\
(n=1134)(57 \%)\end{array}$ & $\begin{array}{l}\text { Females } \\
(n=868)(43 \%)\end{array}$ \\
\hline Age, years, $\pm S D$ & $66.9 \pm 9.5$ & $69.0 \pm 9.1$ & $64.3 \pm 9.2 *$ \\
\hline Weight, kg & $71.9 \pm 16.5$ & $77.2 \pm 15.9$ & $65.0 \pm 14.7^{*}$ \\
\hline Height, m & $1.68 \pm 0.9$ & $1.73 \pm 0.7$ & $1.62 \pm 0.6^{*}$ \\
\hline BMI, $\mathrm{kg} / \mathrm{m}^{2}$ & $25.4 \pm 5.2$ & $25.8 \pm 4.9$ & $25.0 \pm 5.5^{*}$ \\
\hline FFMI, $\mathrm{kg} / \mathrm{m}^{2}$ & $17.0 \pm 2.4$ & $18.1 \pm 2.2$ & $15.5 \pm 1.8^{*}$ \\
\hline $\mathrm{FEV}_{1}, \%$ predicted & $48.2 \pm 23.2$ & $47.2 \pm 20.7$ & $49.6 \pm 26.1^{*}$ \\
\hline FVC, \%predicted & $95.9 \pm 22.3$ & $93.0 \pm 21.7$ & $99.7 \pm 22.7 *$ \\
\hline $\mathrm{FEV}_{1} / \mathrm{FVC}, \%$ & $39.8 \pm 11.8$ & $38.9 \pm 11.9$ & $40.9 \pm 11.4^{*}$ \\
\hline FRC, \%predicted & $150.1 \pm 35.9$ & $146.3 \pm 37.5$ & $154.9 \pm 33.0 *$ \\
\hline DLCO, \%predicted & $52.9 \pm 18.1$ & $55.3 \pm 30.1$ & $50.9 \pm 25.7^{*}$ \\
\hline $\mathrm{PO}_{2}, \mathrm{kPa}$ & $9.8 \pm 2.3$ & $9.8 \pm 2.2$ & $9.7 \pm 2.4$ \\
\hline $\mathrm{PCO}_{2}, \mathrm{kPa}$ & $5.3 \pm 1.4$ & $5.2 \pm 1.2$ & $5.4 \pm 1.5$ \\
\hline MRC dyspnea grade & $3.3 \pm 1.1$ & $3.3 \pm 1.1$ & $3.4 \pm 1.0$ \\
\hline GOLD stage I & $96 \pm 4.8$ & $48 \pm 4.2$ & $48 \pm 5.5$ \\
\hline GOLD stage II & $649 \pm 32.4$ & $362 \pm 31.9$ & $287 \pm 33.1$ \\
\hline GOLD stage III & $730 \pm 36.5$ & $419 \pm 36.9$ & $311 \pm 35.8$ \\
\hline GOLD stage IV & $527 \pm 26.3$ & $305 \pm 26.9$ & $222 \pm 25.6$ \\
\hline Receiving LTOT, n, (\%) & $408(20.4)$ & $230 \pm 20.3$ & $178 \pm 20.5$ \\
\hline
\end{tabular}

Abbreviations: BMI: Body Mass Index, FFMI: Fat-Free Mass Index, FEV 1 : Force Expiratory Volume at 1 sec, FVC: Force Vital Capacity, FRC: Functional Residual Capacity, DLCO: Diffusing capacity of the lung for carbon monoxide; $\mathrm{PO}_{2}$ : Partial pressure of Oxygen, $\mathrm{PCO}_{2}$ : Partial pressure of Carbon-dioxide, MRC: Medical Research Council dyspnea scale, LTOT: Long-term oxygen therapy. Average values are shown for exercise tests and 


\section{Chapter 2}

clinical parameters. The values are reported for the entire cohort and then for men and women. Data are mean \pm SD unless specified otherwise. Asterisk denotes significant differences $(p<0.05)$.

\section{Maximal incremental cycle tests}

On average, patients had a limited maximal exercise tolerance. The percent of predicted peak work rate achieved was significantly different between men (48 $\pm 21 \%$ predicted) and women $(67 \pm 31 \%$ predicted; $p<0.001$; Table 2$)$.

\section{Endurance tests}

Average CET- $T_{\text {end }}$ was $271 \pm 166 \mathrm{~s}$. This differed significantly between men (286s) and women $(250 \mathrm{~s} ; \mathrm{p}<0.001)$. Average 6MWD was $438 \pm 113 \mathrm{~m}$. The 6MWD was higher in men compared to women $(443 \mathrm{~m}$ vs $431 \mathrm{~m} ; \mathrm{p}=0.019)$. Then again, 6MWD \% predicted differed between men ( $67 \pm 17 \%$ predicted) and women (72 $\pm 15 \%$ predicted; $p<0.001$; Table 2).

Table 2. Parameters of exercise tests in patients with COPD

\begin{tabular}{|c|c|c|c|}
\hline Characteristics & $\begin{array}{l}\text { All patients } \\
(n=2002)\end{array}$ & $\begin{array}{l}\text { Males } \\
(n=1134)(57 \%)\end{array}$ & $\begin{array}{l}\text { Females } \\
(n=868)(43 \%)\end{array}$ \\
\hline \multicolumn{4}{|l|}{ Maximal incremental cycle test } \\
\hline Work Rate peak, watts, \pm SD & $69 \pm 30$ & $77 \pm 33$ & $60 \pm 23 * *$ \\
\hline Work Rate peak, \%predicted & $56 \pm 27$ & $48 \pm 21$ & $67 \pm 31^{* *}$ \\
\hline VE, \%Max Voluntary Ventilation & $87 \pm 24$ & $86 \pm 23$ & $89 \pm 25$ \\
\hline HR max, \% age-predicted & $81 \pm 12$ & $80 \pm 13$ & $81 \pm 12$ \\
\hline Systolic BP post, $\mathrm{mm} \mathrm{Hg}$ & $176 \pm 33$ & $176 \pm 34$ & $176 \pm 32$ \\
\hline Diastolic BP post, mm Hg & $84 \pm 15$ & $84 \pm 15$ & $84 \pm 14$ \\
\hline Dyspnea (Borg) post, points & $7.2 \pm 2.0$ & $7.1 \pm 2.0$ & $7.2 \pm 2.0$ \\
\hline Leg Fatigue (Borg) post, points & $5.9 \pm 2.6$ & $5.8 \pm 2.5$ & $6.0 \pm 2.6$ \\
\hline \multicolumn{4}{|l|}{ Cycle endurance test (CET) } \\
\hline Work Rate mean, w & $52 \pm 23$ & $58 \pm 25$ & $45 \pm 17 * *$ \\
\hline Endurance cycling time, s & $271 \pm 166$ & $286 \pm 174$ & $250 \pm 153^{* *}$ \\
\hline Dyspnea (Borg) post, points & $5.3 \pm 2.3$ & $5.4 \pm 2.3$ & $5.1 \pm 2.3^{*}$ \\
\hline Leg Fatigue(Borg) post, points & $5.7 \pm 2.2$ & $5.8 \pm 2.2$ & $5.6 \pm 2.1$ \\
\hline \multicolumn{4}{|l|}{ Six-minute walk test (6MWT) } \\
\hline 6MWD, m & $438 \pm 113$ & $443 \pm 120$ & $431 \pm 104^{*}$ \\
\hline 6MWD, \%predicted & $69.0 \pm 16.5$ & $66.9 \pm 17.1$ & $71.7 \pm 15.3^{* *}$ \\
\hline Dyspnea (Borg) post, points & $5.2 \pm 4.4$ & $5.2 \pm 4.2$ & $5.1 \pm 4.7$ \\
\hline Leg Fatigue(Borg) post, points & $4.4 \pm 4.6$ & $4.4 \pm 4.2$ & $4.4 \pm 4.8$ \\
\hline
\end{tabular}

Abbreviations: VE: Expired Ventilation, HR: Heart Rate, BP: Blood Pressure, 6MWD: Six-minute walk distance. Average values are shown for exercise tests and clinical parameters. The values are reported for the entire cohort and then for men and women. Data are mean \pm SD unless specified otherwise. Asterisks denotes significant differences $(* p<0.05 ; * * p<0.001)$. 


\section{Distribution of cycle endurance times and six minute walking distances}

When separated into 1 minute intervals, the frequency distribution curve for CET-T end shows a leftward kurtosis in men and women (Figure $1 \mathrm{~A}$ ). Only 51 , or $2.5 \%$, of patients achieved CET-T end 20 minutes. When separated into $50 \mathrm{~m}$ intervals, the frequency distribution curve for 6MWD shows a rightward kurtosis in men and women (Figure 1B).

\section{Associations}

The CET- end $_{\text {end }}$ and the GMWD were significantly associated in the whole sample, as well as after stratification for gender presenting a moderate positive correlation (Figure 2).

\section{Examination of performance quartiles}

There is clear performance quartile gradation for $\mathrm{FEV}_{1} \%, \mathrm{FVC} \%, \mathrm{CET}_{\mathrm{T}} \mathrm{T}_{\text {end }}$, peak power output of absolute watt and watt \%predicted (sole exception $3^{\text {rd }} / 4^{\text {th }}$ quartile in CET) and the 6MWD with significant differences amongst all quartiles for the exercise performance of CET and the 6MWT (Figure 3). Moreover, there is performance quartile gradation for age with significant differences of age for the $4^{\text {th }}$ and the rest of quartiles for CET and amongst all the quartiles for the 6MWT. Additionally, performance quartile gradation significant differences were detected across the GOLD stages between $1^{\text {st }}$ and $4^{\text {th }}, 1^{\text {st }}$ and $3^{\text {rd }}$, and $2^{\text {nd }}$ and $4^{\text {th }}$ in cycling and amongst all quartiles in 6MWT. The performance quartile gradation for the FRC demonstrated significant differences in all quartiles for CET while a similar pattern was observed for the 6MWT. The performance quartile gradation for the DLCO demonstrated significant differences amongst $1^{\text {st }}$ and $3^{\text {rd }}-4^{\text {th }}, 2^{\text {nd }}$ and $4^{\text {th }}$ quartiles for the CET and 6MWT respectively. Significant difference in BMI were observed only in CET for $1^{\text {st }}$ and $3^{\text {rd }}-4^{\text {th }}, 2^{\text {nd }}$ and $3^{\text {rd }}-4^{\text {th }}$, but only between $1^{\text {st }}$ and $4^{\text {th }}$ in $6 \mathrm{MWT}$. Similarly, FFMI performance quartile gradation was significant for almost all quartiles but no significance was detected for the 6MWT (Figure 3). When performance quartiles were determined separately for men and women, men demonstrated more evident differences in FFMI quartiles compared to women. That fact indicates that FFMI may determine the exercise performance more in men than women with COPD (see supplemental material for details, Tables S1-S2, Figures S2-S3).

\section{Stepwise multiple linear regression}

Reverse stepwise linear regression revealed that age, gender, FFMI, DLCO\%, GOLD stage, Dyspnea and leg fatigue Borg scores at rest independently influence CET-T end (Table 3). Age, BMI, FFMI, FEV $1 \%$, FVC/FEV 1 , GOLD stage, FRC, DLCO, and leg fatigue Borg score at rest were retained in the model when 6MWD was the dependent variable (Table 4). The model for CET was able to account for only $15.1 \%$ of the variability in 


\section{Chapter 2}

cycle endurance time, while that for 6MWT accounted for $34.0 \%$ of the variability in 6MWD.

Table 3. Stepwise linear regression model of endurance cycle time in seconds

\begin{tabular}{lcccc}
\hline \multicolumn{5}{c}{$\mathrm{r}^{2}: 15.1 \%$} \\
\hline Variable & Coefficient & Standard Error & t value & $p$ value \\
\hline Age, years & -3.35 & 0.57 & -5.84 & $<0.001$ \\
Sex, $\mathrm{m} / \mathrm{f}$ & -31.51 & 12.91 & -2.44 & 0.015 \\
FFMI, kg/m & 8.49 & 2.79 & 3.04 & 0.002 \\
GOLD stage & -42.09 & 6.59 & -6.39 & $<0.001$ \\
DLCO, \%predicted & 1.21 & 0.32 & 3.83 & $<0.001$ \\
Dyspnea before test, index & -13.38 & 4.43 & -3.02 & 0.003 \\
Leg fatigue before test, index & -6.96 & 3.52 & -1.98 & 0.048 \\
\hline
\end{tabular}

Multiple linear regression model for parameters predictive of endurance cycle time. Using isolated linear regression determination a stepwise multiple linear regression model was established with an $r^{2}$ of $15.1 \%$.

Table 4. Stepwise linear regression model of Six minute walking distance in meters

\begin{tabular}{lccrc}
\multicolumn{5}{c}{$\mathrm{r}^{2}: 34.0 \%$} \\
\hline Variable & Coefficient & Standard Error & t value & p value \\
\hline Age, years & -3.97 & 0.29 & -13.98 & $<0.001$ \\
BMI, $\mathrm{kg} / \mathrm{m}^{2}$ & -7.48 & 0.75 & -9.87 & $<0.001$ \\
FFMI, kg/m & 8.77 & 1.59 & 5.52 & $<0.001$ \\
FEV $1, \% p r e d i c t e d$ & 1.28 & 0.29 & 4.44 & $<0.001$ \\
FEV 1 /FVC, \% & -1.60 & 0.42 & -3.89 & $<0.001$ \\
GOLD stages & -15.04 & 5.04 & -3.02 & 0.003 \\
FRC, \%predicted & -0.43 & 0.11 & -3.85 & $<0.001$ \\
DLCO, \%predicted & 1.11 & 0.16 & 6.89 & $<0.001$ \\
Leg fatigue before test, index & -15.80 & 1.64 & -9.57 & $<0.001$ \\
\hline
\end{tabular}

Multiple linear regression model for parameters predictive of six minute walk distance. Using isolated linear regression determination a stepwise multiple linear regression model was established with an $r^{2}$ of 34.0. 
A.

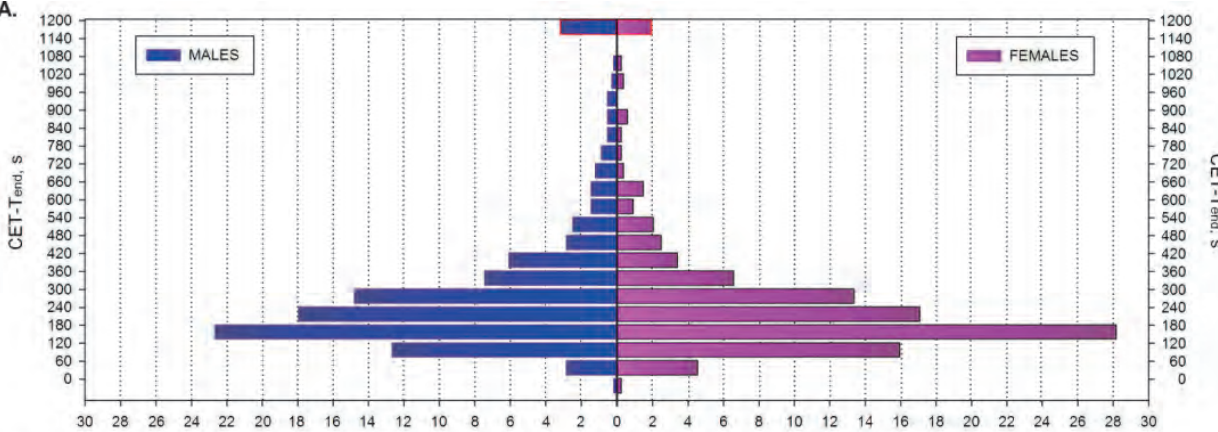

B.

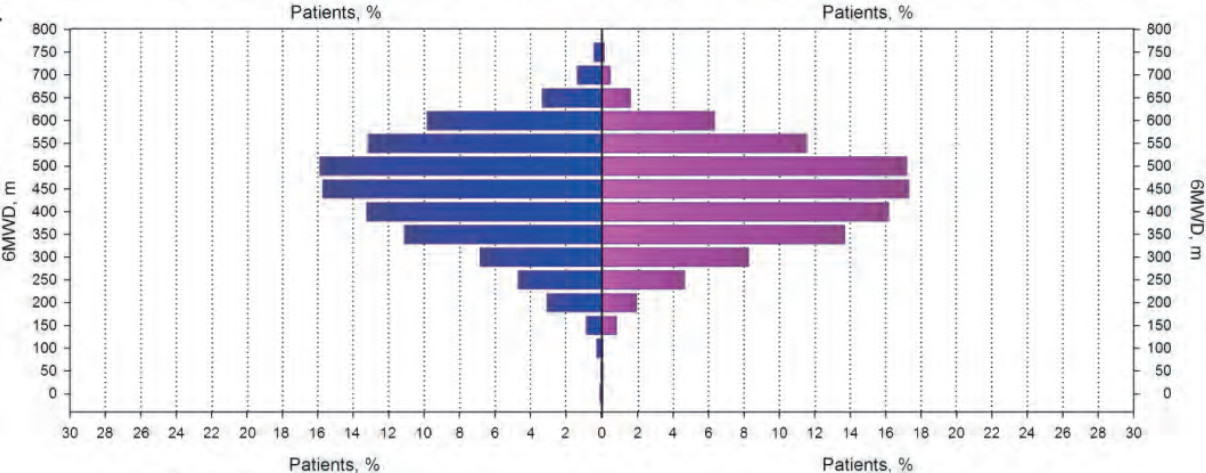

Figure 1. Frequency distribution of CET-Tend and 6MWD in patients with COPD

(A) Frequency distribution of $75 \%$ of peak work rate CET-Tend. The number of individuals for each bin of duration time is represented by the height of each column (y-axis). Each bin represents 1 minute as central value (x-axis). Only 51 (2.5\%) of the 2053 patients who met the inclusion criteria reached the 20 minute maximal duration. (B) Frequency distribution of six minute walking distance (6MWD). The number of individuals for each bin of walking meters is represented by the height of each column (y-axis). Each bin represents 1 minute as central value (x-axis).

A. Total sample

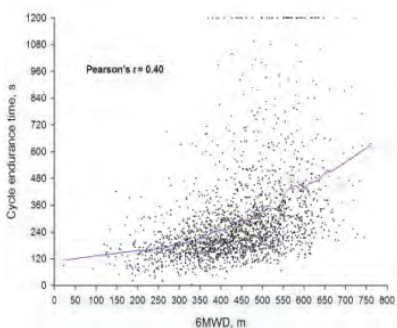

B. Males

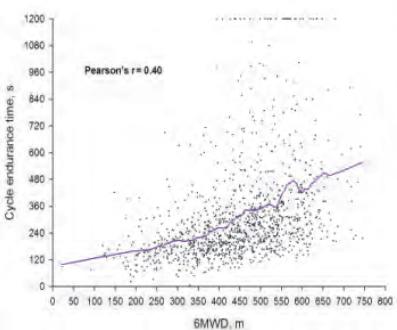

C. Females

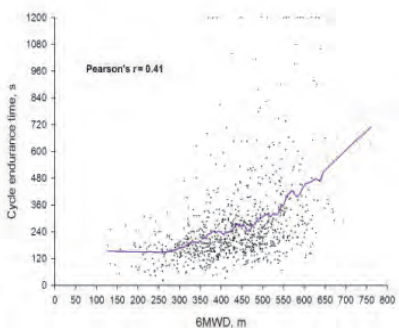

Figure 2. Pearson's correlations between CET-Tend and 6MWD.

A positive moderate correlation was detected in all models between the CET-T end and 6MWD for all patients and stratified by gender. 
Chapter 2
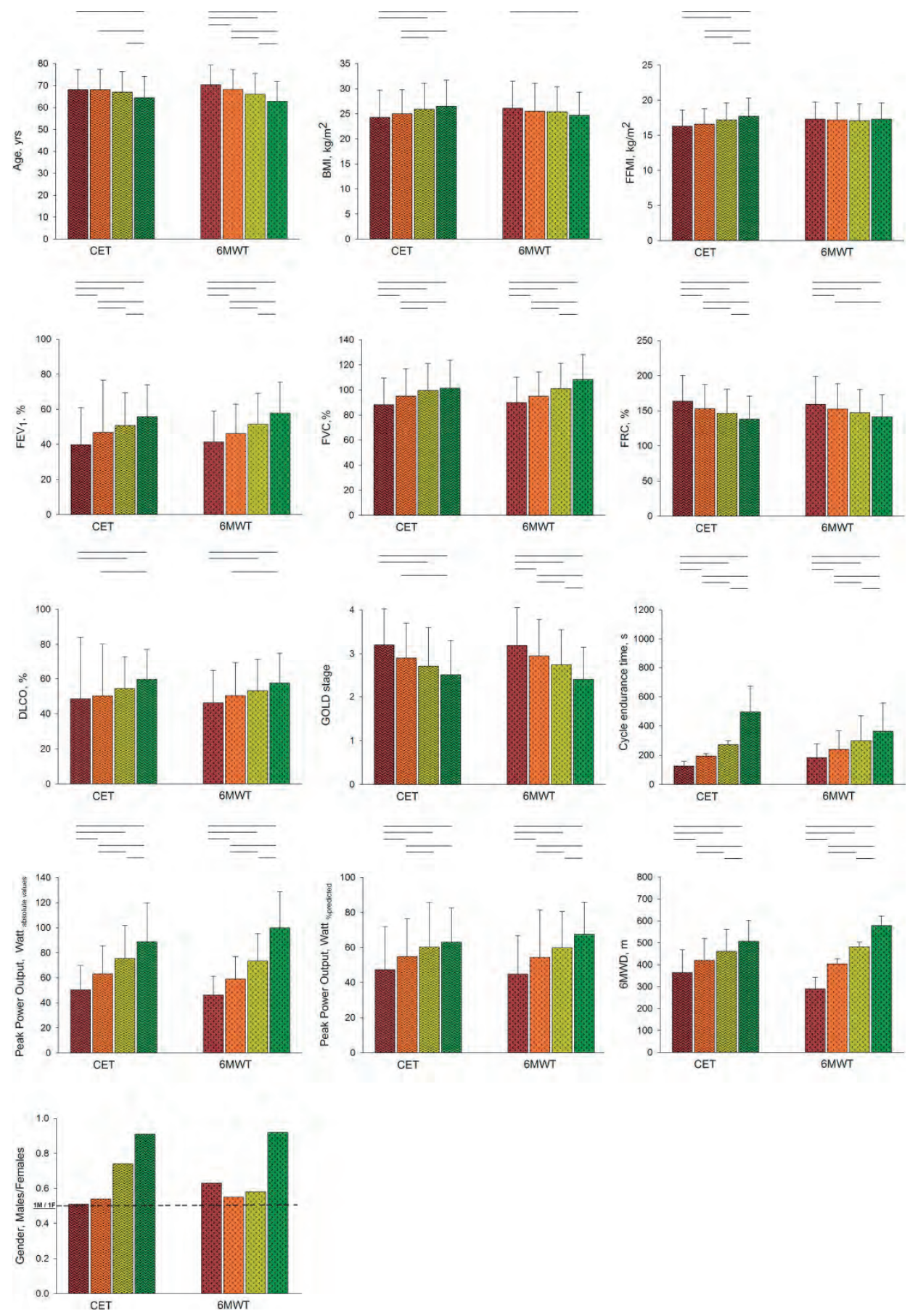

Figure 3. Performance quartile comparisons 
Using the frequency distribution patients were separated into performance quartiles. With the quartiles increasing left to right (CET: quartiles with wave hashing, 6MWT: quartiles with diagonal hashing). Comparisons were then made between the quartiles of CET and between quartiles of 6MWT for all variables. Significant differences are demonstrated by lines above the relevant bars. Dashed line on gender graph represents the point where is an equal number of men and women.

\section{DISCUSSION}

In current study, we aimed to investigate the characteristics of functional exercise performance and the determinants which can influence the exercise performance in CET and the 6MWT in a large cohort of patients with moderate to severe COPD (Table 1). The main finding of this study is that CET-Tend can be determined by different physiological and clinical characteristics in comparison with the 6MWT. Of particular

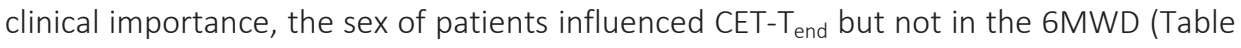
2-4). Moreover, 6MWD seems to be more determined by lung function compared to CET-T $T_{\text {end }}$ (Tables 3-4). Also, a number of independent physiological and clinical characteristics demonstrated a different pattern in performance quartile gradation between CET and 6MWT (Figure 3).

Gender appears to be a strong determinant of CET- end $_{\text {en }}$ in contrast to 6MWD. We found that men on average achieved a higher peak work rate and cycled longer during the CET than women; in spite of the fact the men were older with more impaired lung function (Table 1 and 2). Previous studies have demonstrated that quadriceps muscle strength ${ }^{22}$ and peak $\mathrm{VO}_{2}{ }^{23,24}$ have small reductions in COPD patients over a 1 to 5 year period. Moreover, quadriceps muscle strength is positively related to DLCO in patients with $\mathrm{COPD}^{25}$ and the studied male population had a greater diffusing capacity which could be accountable for the better cycle performance.

When we examined the performance quartiles, there was not as much of a gradation in gender proportion except the $4^{\text {th }}$ quartile amongst the 6MWD performance quartiles compared to that was seen in the CET-T end of performance quartiles. Furthermore, there was a significant difference between all the CET performance quartiles in FFMI, which was not seen 6MWD performance quartiles. However, as would be expected, FFMI was on average lower in the female population compared to men (Table 1). Thus, the question remains whether and to what extent gender differences in CET reflected the lower FFMI in women, or if the FFMI differences were simply a reflection of inherent gender differences. To examine this question we stratified the data into performance quartiles for each gender. We found that the difference in FFMI between performance quartiles was no longer apparent in women except for the $1^{\text {st }}$ and $4^{\text {th }}$ CET performance quartiles (Supplementary figures S1-S2), but still apparent in 
men between all the quartiles in CET and the $1^{\text {st }}$ and $4^{\text {th }}$, and the $1^{\text {st }}$ and $3^{\text {rd }}$ quartiles in the 6MWT. There was also less of a CET quartile gradation in FVC\% and GOLD stage compared to that seen in 6MWD. To further elucidate the importance of gender and FFMI as determinants of test performance we carried out stepwise multiple regressions. The parameters that were retained when CET- end $_{\text {en }}$ was used as the dependent variable were age, gender, FFMI, DLCO\%, GOLD stage, Dyspnea and leg fatigue Borg scores at rest (Table 3). When 6MWD was used as the dependent variable, age, BMI, FFMI, $\mathrm{FEV}_{1} \%, \mathrm{FEV}_{1} / \mathrm{FVC}, \mathrm{FRC} \%$, DLCO\%, GOLD stage and leg fatigue Borg score at rest were retained, but gender was not retained (Table 4). Performing a stepwise multiple regression after stratification by gender, FFMI was not retained in women but it was

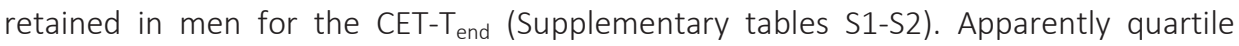
difference in FFMI (Figure 3) can be likely a reflection of an inherent gender differences beyond fat free mass. Thus, it can be concluded that gender is an important

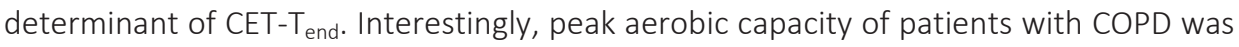
not determined by gender, after correction for isokinetic quadriceps peak torque, DLCO, $\mathrm{FEV}_{1}, \mathrm{BMI}$, age and lower-limb lean muscle mass. ${ }^{26}$

The model for 6MWD was much better at explaining variability in test performance $\left(r^{2}\right.$ of $34.0 \%$ ) compared to that for CET ( $r^{2}$ of $15.1 \%$ ). Other authors have also found it difficult to build a model that is highly predictive of CET performance. ${ }^{12,27}$ This perhaps explains why we do not have a predictive equation for CET-T end yet. It does however suggest that there are determinants of CET which we do not understand. This may reflect the complex nature of exercise intolerance in COPD.

CET-T $T_{\text {end }}$ seems to be influenced in a high extent from the degree of disease severity classified by GOLD stages more than the inter-individual variation of $\mathrm{FEV}_{1}$ or other specific clinical characteristics compared to 6MWT. It is observed that patients across the GOLD stages are characterized by increased physical deconditioning which leads to a progressive deterioration in lower limb muscle function. ${ }^{28}$ The impact of the power/duration relationship on the exercise tolerance indicates that endurance is influenced by the intensity of exercise. ${ }^{29}$ Both exercise tests are sub-maximal, however, the 6MWT is a self-paced while the CET at $75 \%$ is a constant load exercise test. The termination of exercise in CET is more associated to the inability to maintain the constant work load of exercise and thus CET-Tend is associated to an overall impaired health status, sex of patients and physical deconditioning more than specific lung function characteristics compared to the 6MWT.

In contrast to the findings of Neder and colleagues ${ }^{10}$, we found that the vast majority of patients with COPD did not reach the 20 minute time point during a CET at $75 \%$ of peak work rate. This discrepancy between two studies is likely the result of differences in methodology and study populations. We retrospectively examined a clinical cohort of patients who were referred for pulmonary rehabilitation, whereas Neder and colleagues ${ }^{10}$ studied a proscribed, smaller cohort with the intent of defining 
the power duration asymptote. In the present study, CET- Tend $_{\text {varied from } 2}$ to $5 \mathrm{~min}$ for the most of the patients (Figure $1 \mathrm{~A}$ ). This is similar to the findings of Vivodtzev and colleagues $^{12}$ where most of the patients sustained CET for 3 to 5 minutes. Although the proportion of patients with CET-T end 20 minutes was very small (2.5\%), we evaluated the possibility of an underestimation in peak work rate that may result in unduly prolonged CET. Nevertheless, peak heart rate, ventilation and dyspnea scores following a cardiopulmonary exercise test indicated a maximum effort for those patients (Supplementary table S3). Moreover, a quartile gradation of ventilation pattern derived by the maximum cardiopulmonary exercise test (CPET) in cycle ergometer indicates the effort to maintain $\mathrm{O}_{2}$ and $\mathrm{CO}_{2}$ homeostasis and may partly provide an insight of the ventilatory profile in patients of current study (Supplementary figure S3).

Our findings showed a large variation in the CET-T end values (Figure $1 \mathrm{~A}$ ) which is also observed in the 6MWD, though to a lesser extent (Figure 1B.). Therefore, evaluating patients with a CET at $75 \%$ of peak work rate leaves room for demonstrating improvement with an intervention, especially considering that the minimal clinically important difference has been reported to be between 100 and 200 seconds $^{9}$ or $33 \%$ increased from baseline ${ }^{4}$. Although the identical exercise intensity of $75 \%$ of peak work rate, the physiological stress may differ amongst patients and thus the space for improvement through PR exercise modalities can vary. Moreover, in daily life the majority of patients have been adapted to walking but not to cycling exercise and that may results in a greater potential of improvement in CET compared to 6MWT after PR. Nevertheless, O'Donnell and colleagues ${ }^{15}$ demonstrated that CET at 75\% peak work rate was highly repeatable across run-in visits: $7.9+/-4.8$ and $8.4+/-5.1 \mathrm{~min}(R=0.84)$ in 463 COPD patients indicating the appropriateness of CET in the exercise assessment of patients with COPD.

There are methodological considerations to our study. It is a retrospective cohort study which means there may be unidentified confounders. We have no way of knowing why some of the records were incomplete. Surely some may be incomplete due to a failure of the patients to complete the initial pulmonary rehabilitation assessment, or refusal to perform a particular test. Some were also repeat evaluations. Nonetheless the large sample size helps to mitigate some of these potentially confounding factors. Secondly, this is a population of patients who have been referred for pulmonary rehabilitation which could result in a referral bias towards patients with a greater degree of limitation. Regardless, our findings sufficiently demonstrate that performing CET at $75 \%$ of peak work rate is a reasonable effort level for a population of COPD patients being referred for pulmonary rehabilitation. Although a fixed proportion of peak work rate exercise does not provide identical physiological stress amongst patients Furthermore, this study increases our understanding of gender differences in exercise testing and highlights the complex pathophysiology of exercise intolerance in COPD. There have been a number of gender differences reported in COPD. ${ }^{27,30-36}$ Most 


\section{Chapter 2}

intriguingly, it has previously been shown that women have higher symptom scores on the St. George's Respiratory Questionnaire (SGRQ) compared to men. ${ }^{27}$ It may therefore be that compared to $6 \mathrm{MWT}$, which simulates a more routine daily activity, cycle ergometry evokes more symptoms in women compared to men resulting in reduced CET- $T_{\text {end }}$ for women. This could be a reflection of the fact that most patients with COPD, even in the Netherlands, do not routinely ride a bicycle, and thereby cycling may evoke more dyspnea because of its unfamiliarity. In lieu of predictive equations, this gender difference in CET should be taken into consideration and used to temper the interpretation of CET. In summary, CET at $75 \%$ of peak work rate appears to be a useful clinical exercise tool in COPD. There is plenty of room to demonstrate improvement with an intervention and it measures a different aspect of exercise tolerance compared to 6MWT. The fact that the determinants of CET- $T_{\text {end }}$ are not reducible to a model built from a handful of clinical parameters also speaks to the importance of CET-T end as an independent measurement before and after pulmonary rehabilitation. Furthermore, CET reflects gender differences in COPD, an aspect of the disease that is gaining increasing recognition.

\section{CONCLUSION}

CET and 6MWT are not interchangeable exercise tests. CET- end $_{\text {en }}$ is a valuable outcome of CET as it is related to multiple clinical aspects of disease severity in COPD. Gender difference should temper the interpretation of CET. BMI, FEV 1 and FRC influence the walking performance of $6 \mathrm{MWT}$ rather than the CET. Although the fixed proportion of workload capacity does not provide the same physiological stress among patients, improvement in CET-T end after pulmonary rehabilitation can reflect a total improvement of clinical status in patients with COPD. 


\section{REFERENCES}

1. Ross RM. ATS/ACCP statement on cardiopulmonary exercise testing. Am J Respir Crit Care Med. 2003; 167: 1451

2. Spruit MA, Singh SJ, Garvey C, et al. An official American Thoracic Society/European Respiratory Society statement: key concepts and advances in pulmonary rehabilitation. Am J Respir Crit Care Med. 2013; 188: e13-64.

3. Cooper CB, Abrazado M, Legg D and Kesten S. Development and implementation of treadmill exercise testing protocols in COPD. Int J Chron Obstruct Pulmon Dis. 2010; 5: 375-85.

4. Puente-Maestu L, Villar F, de Miguel J, et al. Clinical relevance of constant power exercise duration changes in COPD. Eur Respir J. 2009; 34: 340-5.

5. Gloeckl R, Marinov $B$ and Pitta F. Practical recommendations for exercise training in patients with COPD. Eur Respir J. 2013; 22: 178-86.

6. Spruit MA, Polkey MI, Celli B, et al. Predicting outcomes from 6-minute walk distance in chronic obstructive pulmonary disease. J Am Med Dir Assoc. 2012; 13: 291-7.

7. Polkey MI, Spruit MA, Wouters E, Edwards LD, Tal-Singer R and Celli B. Reply: minimal or maximal clinically important difference: using death to define MCID. Am J Respir Crit Care Med. 2013; 187: 1392.

8. ATS. ATS statement: guidelines for the six-minute walk test. Am J Respir Crit Care Med. 2002; 166: 111-7.

9. Lacasse Y, Martin S, Lasserson TJ and Goldstein RS. Meta-analysis of respiratory rehabilitation in chronic obstructive pulmonary disease. A Cochrane systematic review. Eura Medicophys. 2007; 43: 475-85.

10. Neder JA, Jones PW, Nery LE and Whipp BJ. Determinants of the exercise endurance capacity in patients with chronic obstructive pulmonary disease. The power-duration relationship. Am J Respir Crit Care Med. 2000; 162: 497-504.

11. van 't Hul A, Gosselink R and Kwakkel G. Constant-load cycle endurance performance: test-retest reliability and validity in patients with COPD. J Cardiopulm Rehabil. 2003; 23: 143-50.

12. Vivodtzev I, Gagnon P, Pepin V, et al. Physiological correlates of endurance time variability during constant-workrate cycling exercise in patients with COPD. PLoS One. 2011; 6: e17007.

13. ATS/ACCP. ATS/ACCP Statement on cardiopulmonary exercise testing. Am J Respir Crit Care Med. 2003; 167: 211-77.

14. Borel B, Provencher S, Saey D and Maltais F. Responsiveness of Various Exercise-Testing Protocols to Therapeutic Interventions in COPD. Pulm Med. 2013; 2013: 410748.

15. O'Donnell DE, Travers J, Webb KA, et al. Reliability of ventilatory parameters during cycle ergometry in multicentre trials in COPD. Eur Respir J. 2009; 34: 866-74.

16. Casas A, Vilaro J, Rabinovich R, et al. Encouraged 6-min walking test indicates maximum sustainable exercise in COPD patients. Chest. 2005; 128: 55-61.

17. Man WD, Soliman MG, Gearing J, et al. Symptoms and quadriceps fatigability after walking and cycling in chronic obstructive pulmonary disease. Am J Respir Crit Care Med. 2003; 168: 562-7.

18. Palange $\mathrm{P}$, Forte $\mathrm{S}$, Onorati $\mathrm{P}$, Manfredi F, Serra $\mathrm{P}$ and Carlone S. Ventilatory and metabolic adaptations to walking and cycling in patients with COPD. J App/ Physiol (1985). 2000; 88: 1715-20.

19. Torres JPDE, Casanova C, Garcini AM, Aguirre-Jaime A and Celli B. Gender and respiratory factors associated with dyspnea in chronic obstructive pulmonary disease. Respir Res. 2007; 8: 18.

20. Spruit MA, Vanderhoven-Augustin I, Janssen PP and Wouters EF. Integration of pulmonary rehabilitation in COPD. Lancet. 2008; 371: 12-3.

21. Hernandes NA, Wouters EF, Meijer K, Annegarn J, Pitta F and Spruit MA. Reproducibility of 6-minute walking test in patients with COPD. Eur Respir J. 2011; 38: 261-7.

22. Hopkinson NS, Tennant RC, Dayer MJ, et al. A prospective study of decline in fat free mass and skeletal muscle strength in chronic obstructive pulmonary disease. Respir Res. 2007; 8: 25.

23. Oga T, Nishimura K, Tsukino M, Sato S, Hajiro T and Mishima M. Exercise capacity deterioration in patients with COPD: Iongitudinal evaluation over 5 years. Chest. 2005; 128: 62-9. 


\section{Chapter 2}

24. Oga T, Nishimura K, Tsukino M, Sato S, Hajiro T and Mishima M. Longitudinal deteriorations in patient reported outcomes in patients with COPD. Respir Med. 2007; 101: 146-53.

25. Spruit MA, Gosselink R, Troosters $T$, et al. Muscle force during an acute exacerbation in hospitalised patients with COPD and its relationship with CXCL8 and IGF-I. Thorax. 2003; 58: 752-6.

26. Spruit MA, Franssen FM, Rutten EP, Wagers SS and Wouters EF. Age-graded reductions in quadriceps muscle strength and peak aerobic capacity in COPD. Rev Bras Fisioter. 2012; 16: 148-56.

27. De Torres JP, Casanova C, Hernandez C, Abreu J, Aguirre-Jaime A and Celli BR. Gender and COPD in patients attending a pulmonary clinic. Chest. 2005; 128: 2012-6.

28. Mador MJ, Kufel TJ and Pineda L. Quadriceps fatigue after cycle exercise in patients with chronic obstructive pulmonary disease. Am J Respir Crit Care Med. 2000; 161: 447-53.

29. Dolmage TE, Evans RA, Hill K, Blouin M, Brooks D and Goldstein RS. The effect of pulmonary rehabilitation on critical walk speed in patients with COPD: a comparison with self-paced walks. Chest. 2012; 141: 413-9.

30. Connett JE, Murray RP, Buist AS, et al. Changes in smoking status affect women more than men: results of the Lung Health Study. Am J Epidemiol. 2003; 157: 973-9.

31. Gan WQ, Man SF, Postma DS, Camp P and Sin DD. Female smokers beyond the perimenopausal period are at increased risk of chronic obstructive pulmonary disease: a systematic review and meta-analysis. Respir Res. 2006; 7: 52.

32. Hsia D, Casaburi R, Pradhan A, Torres E and Porszasz J. Physiological responses to linear treadmill and cycle ergometer exercise in COPD. Eur Respir J. 2009; 34: 605-15.

33. Silverman EK, Weiss ST, Drazen JM, et al. Gender-related differences in severe, early-onset chronic obstructive pulmonary disease. Am J Respir Crit Care Med. 2000; 162: 2152-8.

34. ACSM. American College of Sports Medicine position stand. Progression models in resistance training for healthy adults. Med Sci Sports Exerc. 2009; 41: 687-708.

35. Xu X, Weiss ST, Rijcken B and Schouten JP. Smoking, changes in smoking habits, and rate of decline in $\mathrm{FEV}_{1}$ : new insight into gender differences. Eur Respir J. 1994; 7: 1056-61.

36. Klijn $P$, van Keimpema $A$, Legemaat $M$, Gosselink $R$ and van Stel $H$. Nonlinear exercise training in advanced chronic obstructive pulmonary disease is superior to traditional exercise training. A randomized trial. Am J Respir Crit Care Med. 2013; 188: 193-200. 


\section{SUPPLEMENTAL MATERIAL}

Table S1. Stepwise linear regression model of CET- end $_{\text {end }}$

\begin{tabular}{lcccc}
\hline \multicolumn{5}{c}{$r^{2}: 17.2 \%$} \\
\hline Variable & Coefficient & Standard Error & t value & $p$ value \\
\hline MALES & & & & \\
Age, years & -2.74 & 0.78 & -3.52 & $<0.001$ \\
FFMI, kg/m ${ }^{2}$ & 13.00 & 3.56 & 3.65 & $<0.001$ \\
GOLD stage & -44.24 & 8.91 & -4.97 & $<0.001$ \\
DLCO, \%predicted & 1.25 & 0.42 & 2.98 & 0.003 \\
Dyspnea before test, index & -21.9 & 4.73 & -4.64 & $<0.001$ \\
\hline & & $r^{2}: 11.0 \%$ & & \\
\hline FEMALES & & & & $<0.001$ \\
Age, years & -3.99 & 0.84 & -4.74 & $<0.001$ \\
FEV ${ }_{1}, \%$ predicted & 1.79 & 0.44 & 4.04 & 0.024 \\
DLCO, \%predicted & 1.06 & 0.47 & 2.26 & 0.015 \\
Leg fatigue before test, index & -12.01 & 4.91 & -2.45 & \\
\hline
\end{tabular}

Abbreviations: FFMI: Fat-Free Mass Index, FEV ${ }_{1}$ : Force Expiratory Volume at $1 \mathrm{sec}$, DLCO: Diffusing capacity of the lung for carbon monoxide. Multiple linear regression model for parameters predictive of CET-Tend (in seconds) in males and females.

Table S2. Stepwise linear regression model of Six minute walking distance in meters

\begin{tabular}{|c|c|c|c|c|}
\hline \multicolumn{5}{|c|}{$r^{2}: 32.5 \%$} \\
\hline Variable & Coefficient & Standard Error & t value & $\mathrm{p}$ value \\
\hline \multicolumn{5}{|l|}{ MALES } \\
\hline Age, years & -3.79 & 0.41 & -9.22 & $<0.001$ \\
\hline BMI, $\mathrm{kg} / \mathrm{m}^{2}$ & -3.46 & 0.81 & -4.27 & $<0.001$ \\
\hline $\mathrm{FEV}_{1}, \%$ predicted & 1.72 & 0.42 & 4.11 & $<0.001$ \\
\hline $\mathrm{FEV}_{1} / \mathrm{FVC}, \%$ & -1.25 & 0.55 & -2.26 & 0.024 \\
\hline GOLD stage & -16.39 & 7.36 & -2.23 & 0.026 \\
\hline DLCO, \%predicted & 1.26 & 0.22 & 5.77 & $<0.001$ \\
\hline Leg fatigue before test, index & -19.28 & 2.32 & -8.31 & $<0.001$ \\
\hline \multicolumn{5}{|c|}{$r^{2}: 35.9 \%$} \\
\hline \multicolumn{5}{|l|}{ FEMALES } \\
\hline Age, years & -4.06 & 0.39 & -10.43 & $<0.001$ \\
\hline $\mathrm{BMI}, \mathrm{kg} / \mathrm{m}^{2}$ & -4.92 & 0.69 & -7.19 & $<0.001$ \\
\hline $\mathrm{FEV}_{1}, \%$ predicted & 0.69 & 0.30 & 2.28 & 0.023 \\
\hline GOLD stage & -16.04 & 6.61 & -2.43 & 0.016 \\
\hline DLCO, \%predicted & 1.01 & 0.22 & 4.50 & $<0.001$ \\
\hline Leg fatigue before test, index & -12.61 & 2.27 & -5.55 & $<0.001$ \\
\hline
\end{tabular}

Abbreviations: BMI: Body Mass Index, FEV 1 : Force Expiratory Volume at 1 sec, FVC: Force Vital Capacity, FRC: Functional Residual Capacity, DLCO: Diffusing capacity of the lung for carbon monoxide. Multiple linear regression model for parameters predictive of 6MWD (in meters) in males and females. 


\section{Chapter 2}

Table S3. Evidences of maximum CPET in 51 patients with CET $\geq 20 \mathrm{~min}$

\begin{tabular}{|c|c|c|}
\hline HRmax $\geq 80 \%$ predicted & VEmax $\geq 85 \% \mathrm{MVV}$ & Dyspnea and/or leg fatigue $\geq 7$ \\
\hline$\downarrow$ & $\downarrow$ & $\downarrow$ \\
\hline $\mathrm{N}=31(61 \%)$ patients & $\mathrm{N}=18(35 \%)$ patients & $\mathrm{N}=43(84 \%)$ patients \\
\hline Of rest patients: $\mathrm{N}=20$ (39\%) & Of rest patients: $\mathrm{N}=33(65 \%)$ & Of rest patients: $\mathrm{N}=8(16 \%)$ \\
\hline - 1 (2\%) had only VEmax $\geq 85 \%$ & - 5 (10\%) had only HRmax & - 1 (2\%) had only VEmax $\geq 85 \%$ MVV \\
\hline MVV & $\geq 80 \%$ pred. & - $5(10 \%)$ had only HRmax \\
\hline - 12 (23\%) had only dyspnea & - 13 (26\%) had only dyspnea and/or & $\geq 80 \%$ pred. \\
\hline and/or leg fatigue $\geq 7$ by BORG & leg fatigue $\geq 7$ by BORG & - 2 (4\%) had both VEmax $\geq 85 \% \mathrm{MVV}$ \\
\hline - 7 (14\%) had both VEmax 85\% & - 15 (29\%) had both HRmax & \& HRmax $\geq 80 \%$ predicted \\
\hline MVV \& dyspnea and/or leg & $\geq 80 \%$ pred. \& dyspnea and/or leg & \\
\hline fatigue by BORG scale & fatigue by BORG scale & \\
\hline
\end{tabular}

Abbreviations: HR: Heart Rate, VE: Expired Ventilation, MVV: Maximal Voluntary Ventilation. Percentages of patients who reached a high threshold value in heart rate (HRmax) and/or ventilation (VEmax) and/or Borg scores (Dyspnea/Leg-fatigue) as evidence of an achieved maximum effort during the maximum cardiopulmonary exercise test in cycle ergometer. 

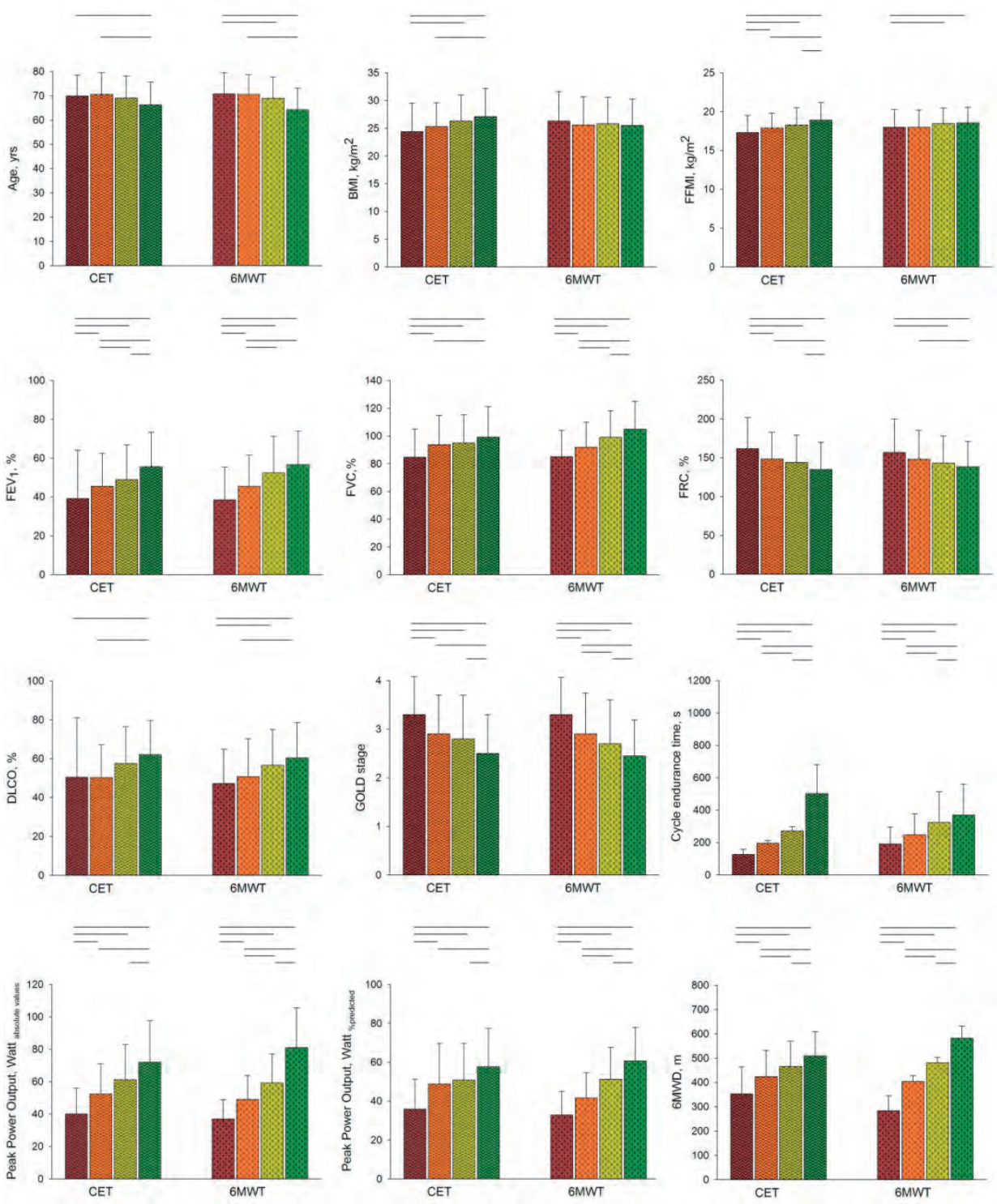

Figure S1. Performance quartile comparisons of men

Using the frequency distribution 1134 male patients was categorized into performance quartiles. With the quartiles increasing left to right (CET: quartiles with wave hashing, 6MWT: quartiles with diagonal hashing). Comparisons were then made between the quartiles of CET and between quartiles of 6MWT for all variables. Significant differences are demonstrated by lines above the relevant bars. 

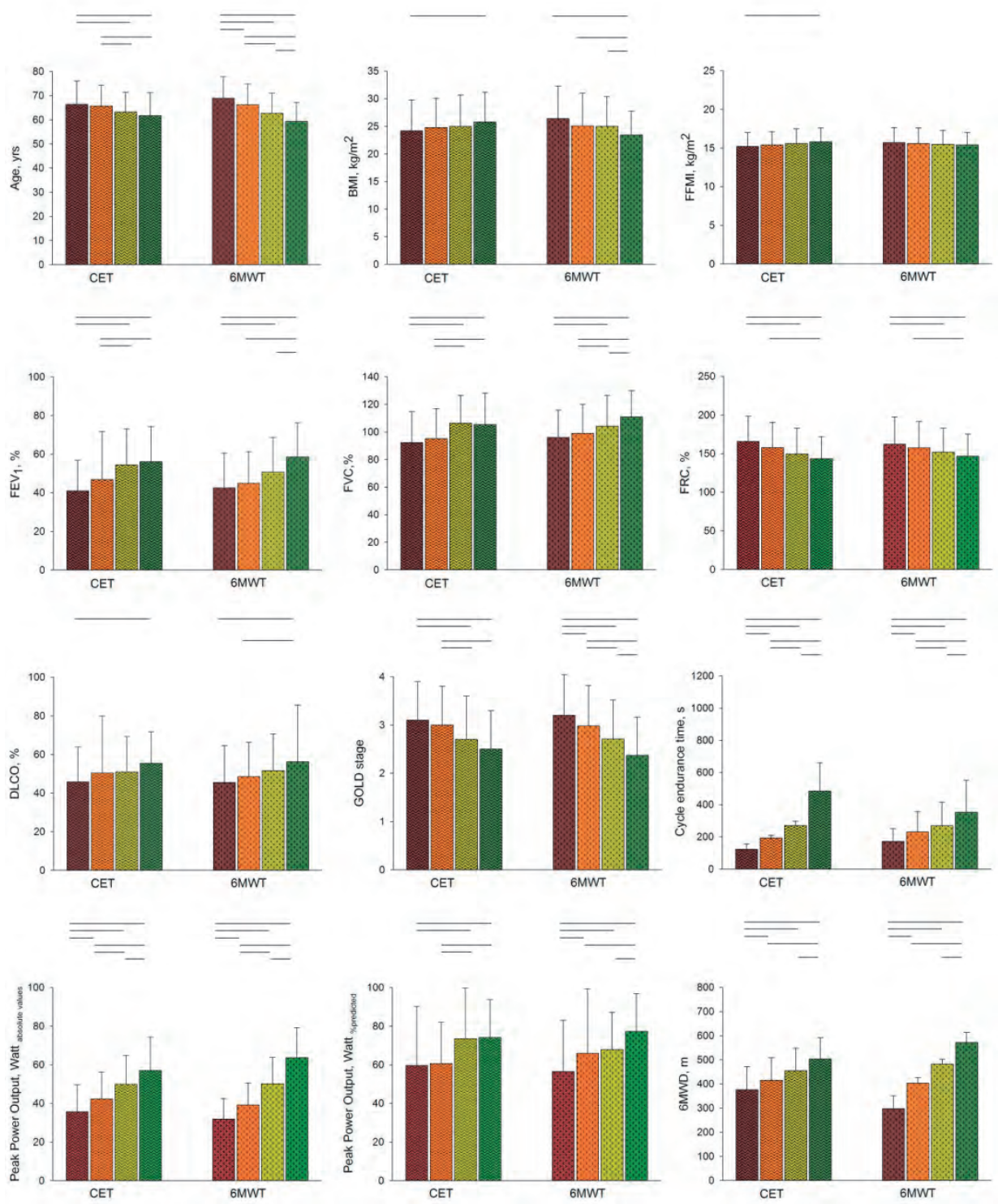

Figure S2. Performance quartile comparisons of women

Using the frequency distribution 868 female patients was categorized into performance quartiles. With the quartiles increasing left to right (CET: quartiles with wave hashing, 6MWT: quartiles with diagonal hashing). Comparisons were then made between the quartiles of CET and between quartiles of 6MWT for all variables. Significant differences are demonstrated by lines above the relevant bars. 

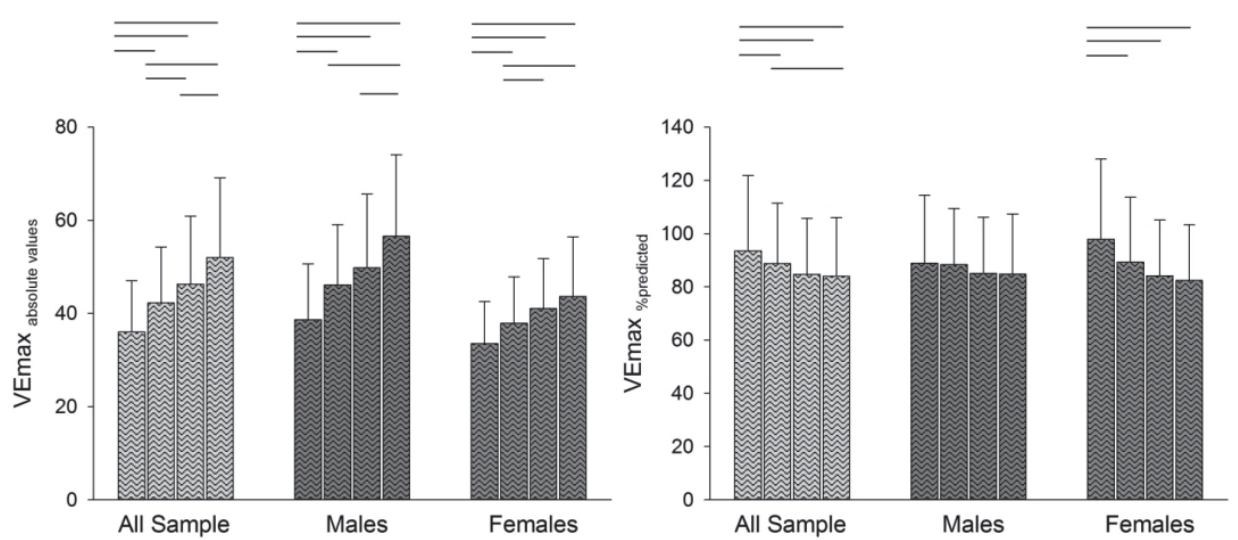

Figure S3. Performance quartile comparisons of CPET

Using the frequency distribution patients were separated into performance quartiles. Quartiles were increasing from left to right (Quartiles with wave hashing for all samples, males and females). Comparisons were then made between the quartiles of maximum cardiopulmonary exercise test (CPET) in cycle ergometer for the achieved maximum ventilation (VEmax) expressed as absolute values and percentage of predicted. Significant differences are demonstrated by lines above the relevant bars. 



\section{Chapter}

Six-minute walk distance in patients with chronic obstructive pulmonary disease: Which reference equations should we use? 


\section{Chapter 3}

\section{ABSTRACT}

\section{Background}

The use of different six-minute walk distance (6MWD) reference equations probably results in different predicted 6MWD reference values. We wished to investigate the impact of several 6MWD reference equations for adults in patients with chronic obstructive pulmonary disease (COPD) and factors accountable for different 6MWD \%predicted values.

\section{Methods}

Twenty-two 6MWD reference equations were applied to a dataset of 2757 patients with COPD. The predicted 6MWD reference value of Troosters and colleagues was used as point of reference.

\section{Results}

Four out of 21 remaining equations resulted in comparable 6MWD \%predicted; sixteen equations resulted in significantly higher 6MWD \%predicted; and one equation resulted in a significantly lower 6MWD \%predicted. Similar differences in 6MWD \%predicted were observed after stratification by sex. Body mass index and global initiative for chronic obstructive lung disease (GOLD) stages classification demonstrated varying results within and between the groups; 9 out of 21 equations resulted in comparable 6MWD \%predicted in underweight patients but only 1 equation demonstrated comparable result in obese. Eight equations in GOLD I, whilst 5 out of 21 equations in GOLD IV resulted in comparable 6MWD \%predicted.

\section{Conclusions}

Existing 6MWD reference equations will give varying results. The choice of 6MWD reference equation should consider the consistency of 6-min walk test operating procedures and at least be specific for the country/region of origin. 


\section{INTRODUCTION}

The six-minute walk test (6MWT) can be used to assess functional exercise capacity, treatment efficacy and prognosis in chronic respiratory disease. ${ }^{1-4} \mathrm{New}$ insights of COPD affliction, which include cardiac and peripheral muscle wasting effects, provide an additional value in the assessment of functional exercise capacity measured by the 6MWT. ${ }^{5}$ The 6MWT elicit high but submaximal cardiorespiratory responses that mimic day life activities. ${ }^{6}$ In some clinical situations, the six-minute walking distance (6MWD) may be a better index of actual patients' ability to perform daily activities in comparison with the peak oxygen uptake. ${ }^{7} 8$ The 6MWD has been indicated for the prediction of hospitalization and mortality in $\mathrm{COPD}^{9}$ and has been also recognized for its predictive value to postoperative outcome in candidates for volume reduction surgery. ${ }^{10}$

Multiple 6MWD reference equations ${ }^{11-30}$ were derived to express the 6MWD as a percentage of these reference values (\% predicted) (see supplemental material for details - Table S1). Adjusting the measured 6MWD for physiological variation using the percentage of walk predicted facilitates the interpretation of the walked 6MWD and help to estimate the degree of exercise tolerance in patients. ${ }^{31}$ Erroneous selection of a reference equation may result in misinterpretation of the levels of exercise capacity or improvements of physical performance after participation in pulmonary rehabilitation programs. $^{30}$ The large number of published 6MWD reference equations and the fact that those equations are derived from healthy individuals with different clinical characteristics and do not consistently employ the same variables makes the choice of 6MWD reference equation often vague.

Several demographic, anthropometric and physiological characteristics can determine the 6MWD in healthy individuals and in patients with COPD. ${ }^{32}$ However, the strongest determinants that can independently affect the 6MWD in healthy adults seem to be the age, height, weight, sex and race and these characteristics have been proposed for clinical use. ${ }^{15,18,26,33}$ Muscle strength, true leg length, symptoms of depression, health-related quality of life impairment and other factors such as the standardization of the 6MWT can influence the 6MWD. ${ }^{11,34-37}$ Therefore, it might be anticipated that the use of different 6MWD reference equations derived by several studies would result in different predicted 6MWD reference values.

Herein, we report a retrospective analysis in a large cohort of patients with COPD who were evaluated at CIRO+ between 1 January 2005 and 1 July 2013. ${ }^{38}$ We assessed the impact of 22 existing 6MWD reference equations in patients with COPD and investigated the factors accountable for different predicted 6MWD reference values using data from 2757 patients with COPD. Furthermore, we wished to identify criteria for the choice of 6MWD reference equation in COPD patients. 


\section{METHODS}

A dataset of patients with COPD $^{39}$ including demographic, anthropometric characteristics, lung function evaluation and 6MWT exercise outcomes was analyzed. All patients performed two 6MWTs according to the American Thoracic Society guidelines ${ }^{7}$, including a practice walk. ${ }^{40}$ The 6MWT with the highest 6MWD was used for further analyses. ${ }^{41}$ During the $6 \mathrm{MWT}$, transcutaneous oxyhaemoglobin saturation $\left(\mathrm{SpO}_{2} \%\right)$ and heart rate (HR) were measured using a pulse oxymeter (Nonin-2500; Nonin Medical Inc., Plymouth, USA). The predicted maximum heart rate (HRmax \%) was derived from the formula $H R \max =220-$ age in years ${ }^{42}$ and the $\Delta$ values of $H R(\Delta H R)$ were calculated by the difference between the $H R$ at the end of the test minus the baseline $H R$.

The 6MWD \% of Troosters and colleagues ${ }^{26}$ was used as a point of reference. These reference values were used in $\mathrm{CIRO}+$ since the last decade, as these reference values were derived from a Belgian sample of healthy elderly subjects with a mean 6MWD of $622 \pm 75 \mathrm{~m}$, which is comparable to the mean 6MWD of Dutch healthy elderly subjects. ${ }^{43}$ All available 6MWD reference equations were applied with the exception of the alternative equation of Dourado and colleagues ${ }^{24}$ which required handgrip strength measurement not an available in our dataset. All statistical analyses were carried out using Sigmaplot Version 11 and Statistical Package for the Social Sciences (SPSS) Version 19.0. Data are presented as mean ( $\pm S D$ ) or proportion, as appropriate. For parametric data, comparisons were made using a two tailed unpaired $t$-test. The 6MWD \% predicted by Troosters and colleagues ${ }^{26}$ was compared with the other reference values using Dunnett's multiple comparisons test. A priori, a two-sided level of significance was set at $p$ value of $<0.05$.

\section{RESULTS}

A total of 22 reference equations were applied to a dataset of 2757 patients with COPD (age range: 38-92 years). Reference equations encompassed race/ethnic differences and variety of technical aspects that can determine the 6MWD (Supplementary figure S1 \& table S2). The clinical features of patients are summarized in Table 1. In brief, patients generally had moderate to very severe COPD and an impaired lung diffusion capacity (DLCO). Men were slightly older compared with women and had more impaired Tiffeneau index (FEV $1 / F V C)$ and lower total lung capacity (TLC), whilst women had worse DLCO\%. On average, the functional exercise capacity was profoundly limited: 422 $\pm 124 \mathrm{~m}$, which equals $68 \pm 18 \%$ of reference values of Troosters and colleagues ${ }^{26}$ (Table $1)$. Four out of 21 remaining equations resulted in comparable 6MWD \%predicted; 16 equations resulted in significantly higher 6MWD \%predicted (all $p<0.05$ ); and 1 equation resulted in a significantly lower 6MWD \% predicted $(p<0.001)$ (Figure 1). Similar 
discrepancies in the 6MWD \%predicted among the equations were observed in patients stratified by sex (Figure 2).

Table 1. Anthropometric and clinical characteristics of patients with COPD

\begin{tabular}{lccc}
\hline Characteristics & $\begin{array}{c}\text { All patients } \\
(\mathrm{n}=2757)\end{array}$ & $\begin{array}{c}\text { Males } \\
(\mathrm{n}=1547)(56 \%)\end{array}$ & $\begin{array}{c}\text { Females } \\
(\mathrm{n}=1210)(44 \%)\end{array}$ \\
\hline Age, years, \pm SD & $67.4 \pm 9.6$ & $69.4 \pm 9.2$ & $64.8 \pm 9.4^{*}$ \\
Height, cm & $168 \pm 9$ & $173 \pm 7$ & $161 \pm 6^{*}$ \\
Weight, kg & $72 \pm 18$ & $77 \pm 17$ & $66 \pm 16^{*}$ \\
BMI, kg/m ${ }^{2}$ & $25.5 \pm 5.6$ & $25.8 \pm 5.2$ & $25.2 \pm 6.1$ \\
FFMI, kg/m ${ }^{2}$ & $17.0 \pm 2.4$ & $18.2 \pm 2.1$ & $15.6 \pm 1.8^{*}$ \\
FEV 1, L & $1.3 \pm 0.6$ & $1.4 \pm 0.6$ & $1.1 \pm 0.5^{*}$ \\
FEV $1, \% p r e d i c t e d$ & $48.0 \pm 19.7$ & $47.4 \pm 19.7$ & $48.8 \pm 19.7$ \\
FVC, \%predicted & $93.9 \pm 22.8$ & $92.6 \pm 32.2$ & $96.7 \pm 23.5^{*}$ \\
Tiffeneau index, \% & $40.8 \pm 13.2$ & $39.9 \pm 13.5$ & $42.0 \pm 12.8^{*}$ \\
TLC, \%predicted & $117.8 \pm 19.2$ & $113.8 \pm 18.5$ & $123.0 \pm 18.7^{*}$ \\
DLCO, \%predicted & $52.8 \pm 19.4$ & $54.2 \pm 20.1$ & $50.5 \pm 18.1^{*}$ \\
SPO,$\%$ & $94.4 \pm 3.6$ & $94.2 \pm 4.1$ & $94.6 \pm 2.6$ \\
mMRC dyspnea grade & $3.4 \pm 1.1$ & $3.3 \pm 1.1$ & $3.5 \pm 1.1^{*}$ \\
$6 M W D$, m & $422 \pm 124$ & $432 \pm 127$ & $409 \pm 120^{*}$ \\
$6 M W D, \% p r e d i c t e d$ & $68 \pm 18$ & $66 \pm 18$ & $70 \pm 17^{*}$
\end{tabular}

Abbreviations: BMI: Body Mass Index, FFMI: Fat-Free Mass Index, FEV 1 : Force Expiratory Volume at 1 sec, FVC: Force Vital Capacity, TLC: Total Lung Capacity, DLCO: Diffusing capacity of the lung for carbon monoxide; $\mathrm{SpO}_{2}$ : Saturation of peripheral oxygen, mMRC: modified Medical Research Council dyspnea scale, 6MWD: Sixminute walk distance. Average values are shown for exercise tests and clinical parameters. The values are reported for the entire cohort and then for men and women. Data are mean \pm SD unless specified otherwise. Asterisk denotes significant differences $(p<0.05)$.

Discrepancies between 6MWD expressed as \%predicted of the reference values of Troosters and colleagues and the 6MWD expressed as \%predicted using the remaining reference equations were also observed after stratification by body mass index (BMI) and the degree of airflow limitation as given by GOLD stages within all groups of patients. Furthermore, differences in the number of 6MWD predicted values with clinically important discrepancies were noticed between the BMI and between the GOLD stages groups. Patients with underweight BMI compared those with obese BMI and patients in GOLD stage I compared those in GOLD stage IV had a smaller number of discrepant 6MWD \%predicted values (Figures 3-4). 


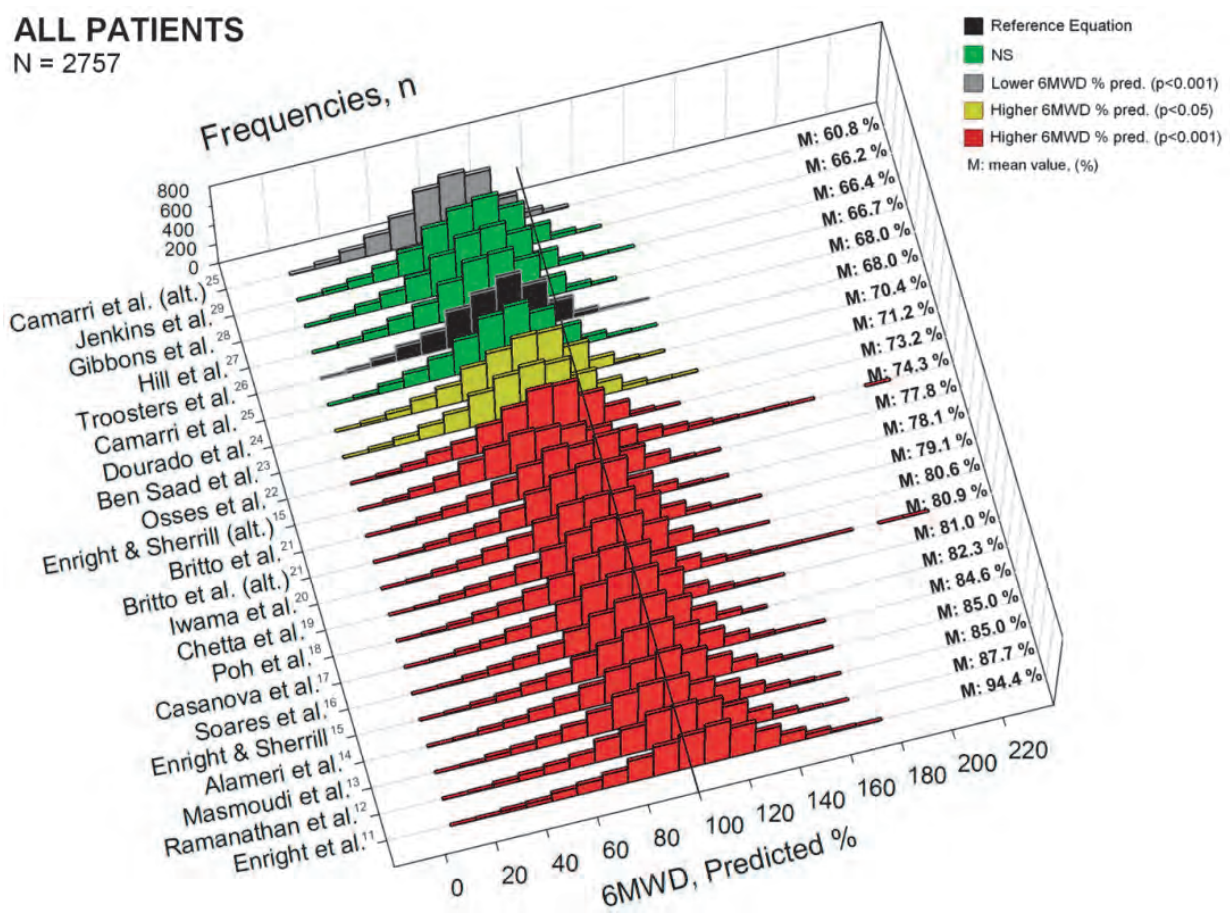

Figure 1. Frequency distribution of estimated 6MWD

The frequency distribution of estimated 6MWD applying the existing equation of healthy individuals to patients with COPD, demonstrated large variation. The numbers on the right are mean values per equation. 


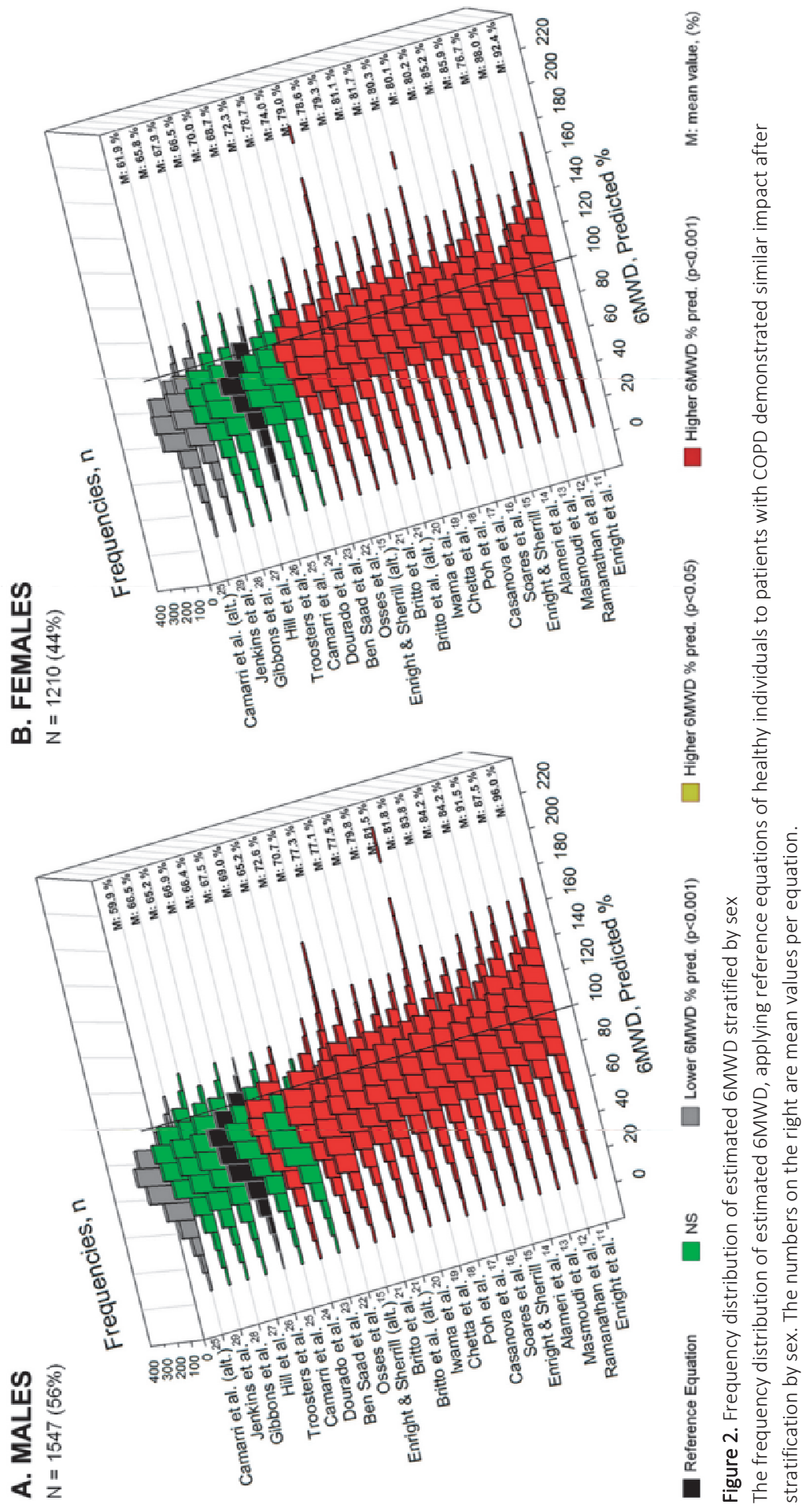



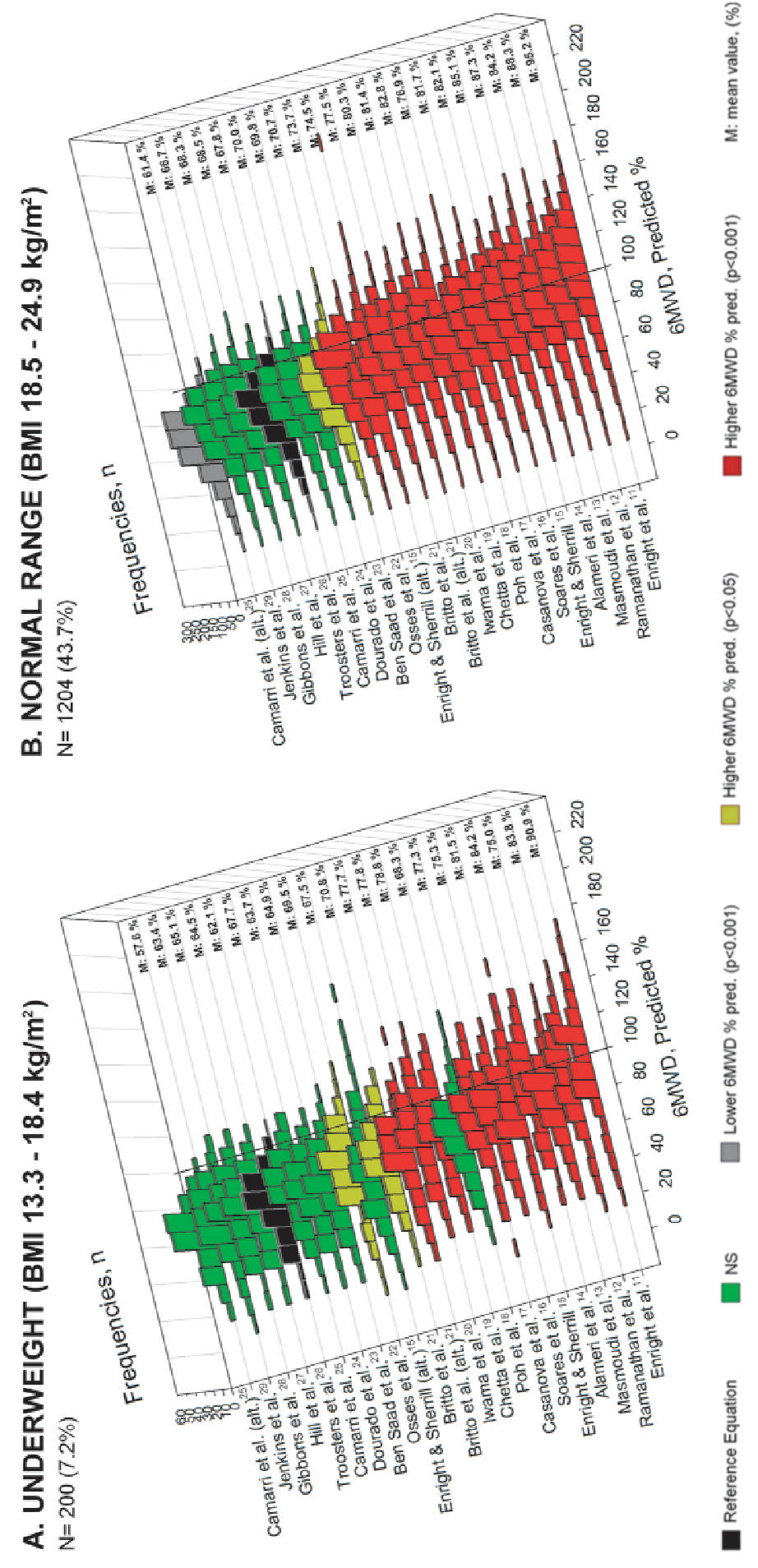


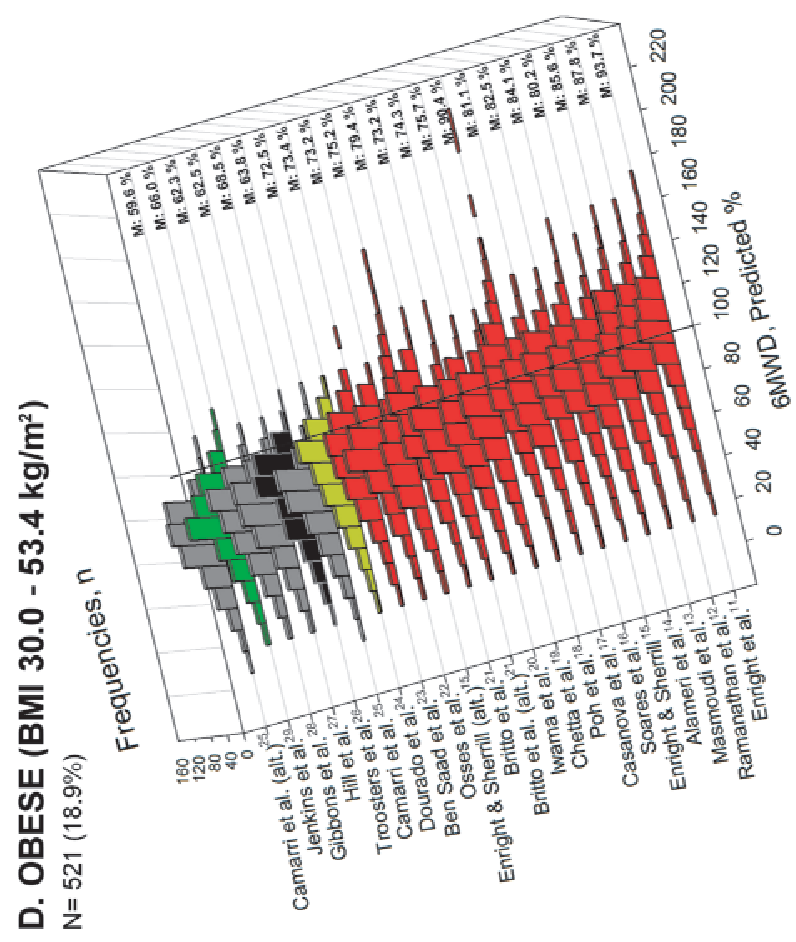

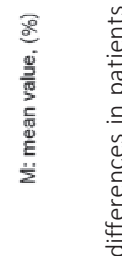

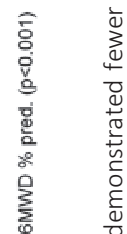

旁 ब

喜 ठ̀

돈

苞

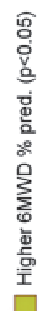

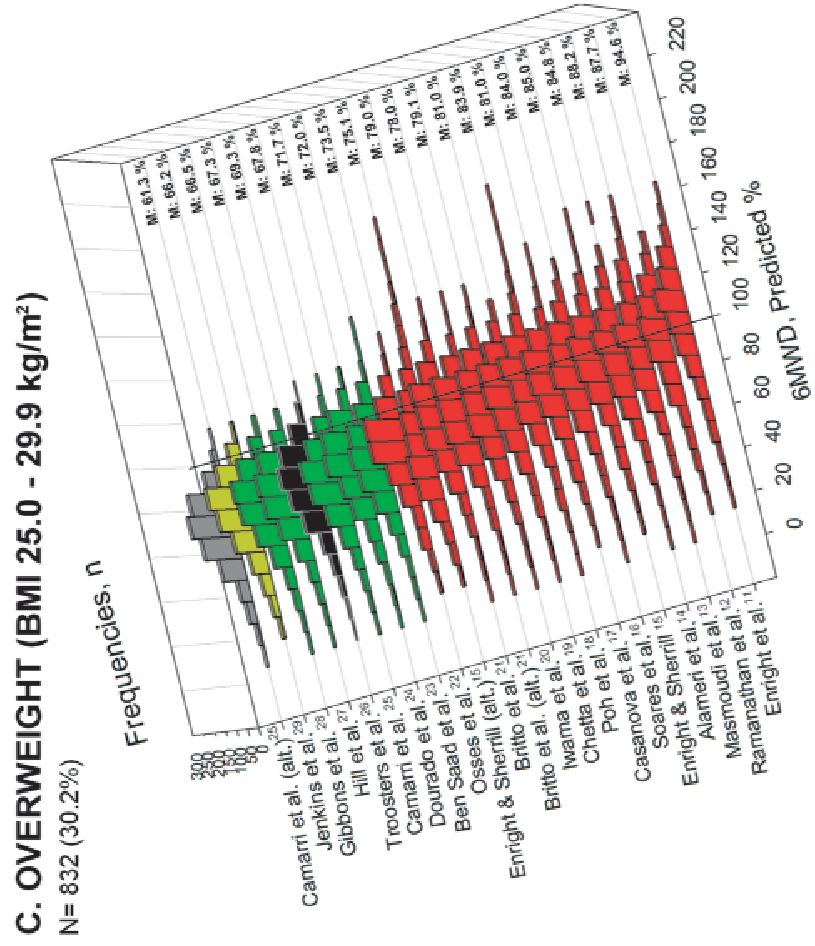

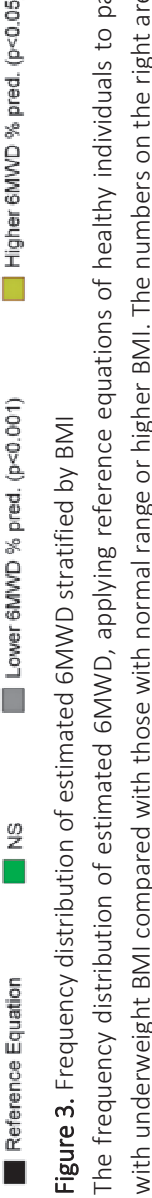




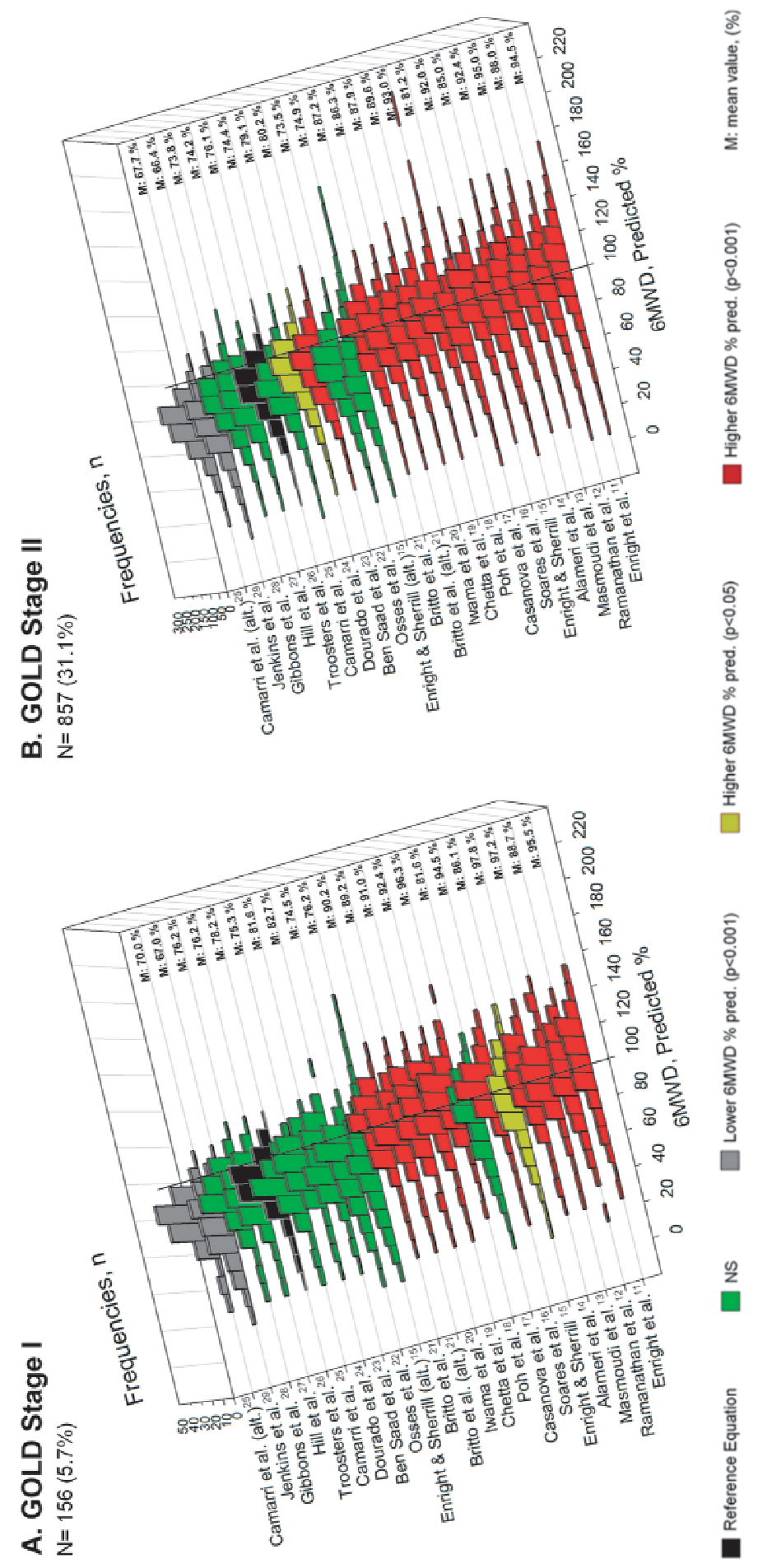




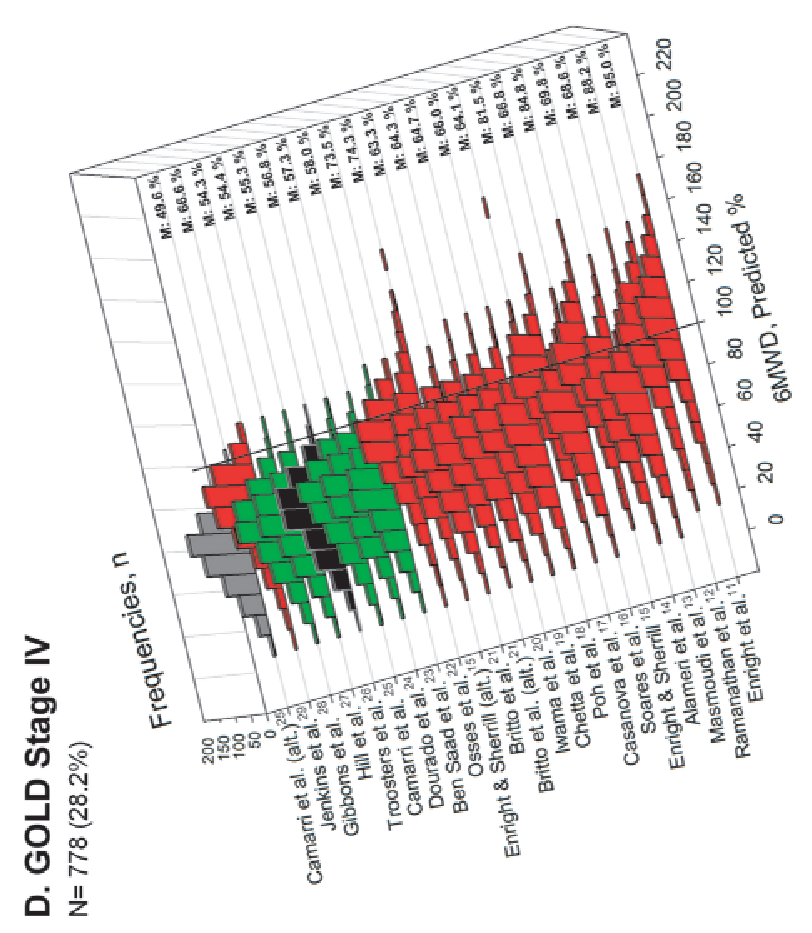

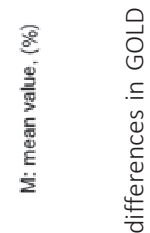

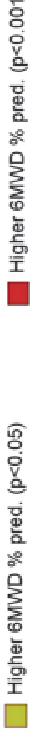
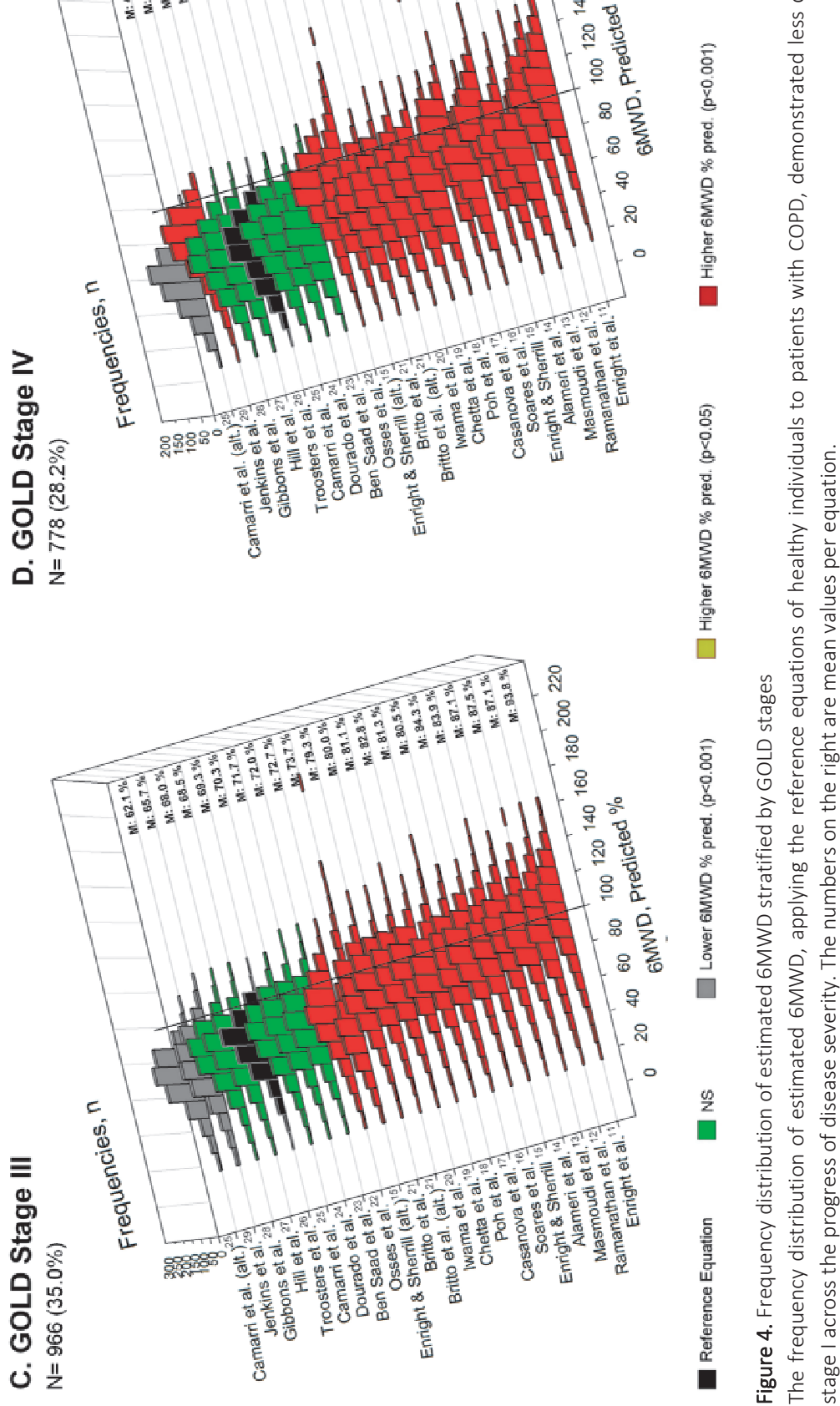


\section{DISCUSSION}

Even though a majority of the existing 6MWD reference equations included similar characteristics, such as sex, age, height, weight or BMI, clear differences were found when applying the existing 6MWD reference equations to a dataset of 2757 patients with COPD (Figure 1). The best choice for the predicted 6MWD reference values probably depends on the country/region of origin of the healthy subjects that were used to develop the 6MWD reference equation. For example, the 6MWD reference equation of Poh and colleagues ${ }^{18}$ resulted in a mean 6MWD of $81 \%$ predicted. This suggests that applying Asian-derived 6MWD \%predicted values to Dutch patients with COPD may result in an overestimation of their true functional capacity. As pointed out by authors, one possible explanation for this discrepancy is the differences in body stature between Asians and Caucasian. Herein, it is worth noting that some countries and regions are very ethnically and racial diverse and reference values derived from mixed-race populations could be recommended. When we applied the reference equation of Enright and colleagues ${ }^{11}$, which is derived from a mixed-race elderly group (aged: $77 \pm 4$ years), true functional capacity of our patients seems to be overestimated compared to the rest of reference values (Figure 1). We assume that it could be partly attributed both to their younger age and to racial differences.

Next to demographic characteristics, a different operating procedure of 6MWT can also affect walking distance and modify the reference values. ${ }^{4}$ For example, in the study of Troosters and colleagues ${ }^{26}$ the length of the corridor of used reference equation was $50 \mathrm{~m}$ which can be considered as long corridor compared to $20 \mathrm{~m}$ corridor of Gibbons and colleagues. ${ }^{28}$ This fact can also result in overestimation of 6MWD \%predicted relative to a number of other reference equations. Accordingly, our triangular track for the 6MWTs of our patients has the characteristics of a long corridor. Therefore, at $\mathrm{CIRO}+$ the predicted reference values of Troosters and colleagues ${ }^{26}$ are used, who studied healthy Belgians 50-85 years of age.

Methodological differences in the 6MWT, with track lengths ranging from $20 \mathrm{~m}$ to $50 \mathrm{~m}$, the number of test repetitions ranging from 1 to 3 , test-retest intervals from $30 \mathrm{~min}$ to $24 \mathrm{~h}$ and variable practices regarding the given test instructions (i.e. "walk at your own pace" or "walk as far as you can") and the encouragement, may partly account for the observed discrepancies in the 6MWD \%predicted values. Sciurba and colleagues $^{44}$ demonstrated that the long compared with short corridors can increase the 6MWD as the number of turns is less and thus the physical effort is less. ${ }^{44}$ In the study of Alameri and colleagues ${ }^{14}$ healthy individuals reached a low average of HRmax (45\%pred.) when instructed to walk at their own pace. ${ }^{14}$ Moreover, the impact of a learning effect on the walking distance of 6MWT has been investigated in COPD. Steven and colleagues ${ }^{45}$ performed three 6MWTs in 21 COPD patients on separate days and observed an average increase of $10 \%$ in the second test and an additional $3 \%$ increase 
in walking distance in the third test compared with first 6MWT. ${ }^{45}$ Such factors are known to have an impact on the measured distance (Supplementary Table S2).

The health status of the "healthy subjects" may also have impacts on findings. For example, the study of Enright and colleagues ${ }^{11}$ included participants with risk factors associated with cardiovascular disease with likely limitations in functional capacity probably resulting in an overestimation of the 6MWD \%predicted when applied to individuals with COPD. The physical condition of the volunteers participated at the several studies should be considered. The HR recorded at the end of the 6MWT can vary substantially in moderate and intense levels of effort in different studies. ${ }^{15,25}$ These facts indicate that differences in characteristics of healthy population, 6MWT protocols and/or different levels of effort during the 6MWT can result in different 6MWD \% predicted values.

On the other side, certain clinical characteristics of patients' populations, where the equations are applied, can also be accountable for the discrepancies in the 6MWD \%predicted values. After stratification for BMI and the degree of airflow limitation as given by GOLD stages, the number of discrepant 6MWD values varied between the groups of BMI and GOLD stages, respectively (Figures 3-4). Applying the 6MWD reference equations to underweight or GOLD stage I groups of patients leads to more consistent 6MWD \%predicted values. Therefore, the choice of 6MWD reference equation in patients with those characteristics (underweight and/or GOLD stage I) may be of less importance. BMI and/or disease severity as given by GOLD stages are major determinants of exercise performance especially in individuals with higher BMI and/or worse COPD. The exclusion of these characteristics from reference equations could account for the discrepancies in 6MWD \%predicted values and that can be more obvious in obese or patients with GOLD stage IV disease. This might explain the larger number of discrepant 6MWD \%predicted values among the reference equations applied in the groups of obese and patients with GOLD stage IV disease compared with the groups of underweight patients with GOLD stage I disease (Figures 3-4). Recently, Capodaglio and colleagues $^{30}$ provided a reference equation specific for obese population and suggested that specific predicted reference values may have the advantage of providing a benchmark for functional capacity assessment and changes monitoring after pulmonary rehabilitation. ${ }^{30}$

Further studies with large cohorts of volunteers in wide range of age would be necessary in order to create a 6MWD reference equation that will be highly representative and applicable to patients with COPD. Specifically, a population-based sample, a large number of healthy subjects in each decade of age, adequate exclusion criteria for poor health of "healthy subjects" and methodological approach based on American Thoracic Society (ATS) guidelines ${ }^{7}$ should be considered for the creation of 6MWD reference equations. Anthropometric and demographic characteristics of healthy individuals recruited for the development of a reference equation should be 


\section{Chapter 3}

ideally matched to the corresponding patients' characteristics in which the equation is being applied. Additionally, the 6MWT operating procedures of studies formulated reference equations should be in line with those operating procedures followed by the population in which the reference equation is being applied. All of these criteria should be considered for the development and the choice of 6MWD reference equation that can provide an instant practical clinical information and interpretation for the levels of exercise capacity. In summary, existing 6MWD reference equations will give varying results in patients with COPD. The choice of 6MWD reference equations should consider the consistency of 6MWT operating procedures and at least be specific for the country/region of origin. This seems of less importance in underweight patients with mild COPD. 


\section{REFERENCES}

1. Spruit MA, Singh SJ, Garvey C, et al. An official American thoracic society/European respiratory society statement: key concepts and advances in pulmonary rehabilitation. Am J Respir Crit Care Med. 2013; 188: e13-e64.

2. Lord SR and Menz HB. Physiologic, psychologic, and health predictors of 6-minute walk performance in older people. Arch Phys Med Rehabil. 2002; 83: 907-11.

3. Singh SJ, Puhan MA, Andrianopoulos V, et al. An official systematic review of the European Respiratory Society/American Thoracic Society: measurement properties of field walking tests in chronic respiratory disease. Eur Respir J. 2014; 44(6): 1447-78.

4. Holland AE, Spruit MA, Troosters T, et al. An official European respiratory society/American thoracic society technical standard: field walking tests in chronic respiratory disease. Eur Respir J. 2014; 44(6): 1428-1446.

5. Chatila WM, Thomashow BM, Minai OA, Criner GJ and Make BJ. Comorbidities in chronic obstructive pulmonary disease. Proc Am Thorac Soc. 2008; 5: 549-55.

6. Holland A, Knapman L, Brazzale DJ, et al. The 6-minute walk test elicits high but submaximal cardiorespiratory responses In interstitial lung disease. Am J Respir Crit Care Med. 2010; 181: A2968.

7. ATS. ATS statement: guidelines for the six-minute walk test. Am J Respir Crit Care Med. 2002; 166: 111-7.

8. Guyatt GH, Townsend M, Keller J, Singer J and Nogradi S. Measuring functional status in chronic lung disease: conclusions from a randomized control trial. Respir Med. 1991; 85 Suppl B: 17-21

9. Spruit MA, Polkey MI, Celli B, et al. Predicting outcomes from 6-minute walk distance in chronic obstructive pulmonary disease. J Am Med Dir Assoc. 2012; 13: 291-7.

10. Szekely LA, Oelberg DA, Wright C, et al. Preoperative predictors of operative morbidity and mortality in COPD patients undergoing bilateral lung volume reduction surgery. Chest. 1997; 111: 550-8.

11. Enright PL, McBurnie MA, Bittner $\mathrm{V}$, et al. The 6-min walk test: a quick measure of functional status in elderly adults. Chest. 2003; 123: 387-98.

12. Palaniappan Ramanathan $\mathrm{R}$ and Chandrasekaran B. Reference equations for 6 -min walk test in healthy Indian subjects (25-80 years). Lung India. 2014; 31: 35-8.

13. Masmoudi K, Aouicha MS, Fki H, Dammak J and Zouari N. [The six minute walk test: which predictive values to apply for Tunisian subjects aged between 40 and 80 years?]. Tunis Med. 2008; 86: 20-6.

14. Alameri H, Al-Majed S and Al-Howaikan A. Six-min walk test in a healthy adult Arab population. Respir Med. 2009; 103: 1041-6.

15. Enright PL and Sherrill DL. Reference equations for the six-minute walk in healthy adults. Am J Respir Crit Care Med. 1998; 158: 1384-7.

16. Soares MR and Pereira CA. Six-minute walk test: reference values for healthy adults in Brazil. J Bras Pneumol. 2011; 37: 576-83.

17. Casanova C, Celli BR, Barria P, et al. The 6-min walk distance in healthy subjects: reference standards from seven countries. Eur Respir J. 2011; 37: 150-6.

18. Poh H, Eastwood PR, Cecins NM, Ho KT and Jenkins SC. Six-minute walk distance in healthy Singaporean adults cannot be predicted using reference equations derived from Caucasian populations. Respirology. 2006; 11: 211-6.

19. Chetta A, Zanini A, Pisi G, et al. Reference values for the 6-min walk test in healthy subjects $20-50$ years old. Respir Med. 2006; 100: 1573-8.

20. Iwama AM, Andrade GN, Shima P, Tanni SE, Godoy I and Dourado VZ. The six-minute walk test and body weight-walk distance product in healthy Brazilian subjects. Braz J Med Biol Res. 2009; 42: 1080-5.

21. Britto RR, Probst VS, de Andrade AF, et al. Reference equations for the six-minute walk distance based on a Brazilian multicenter study. Braz J Phys Ther. 2013; 17: 556-63.

22. Osses AR, Yanez VJ, Barria PP, et al. [Reference values for the 6-minutes walking test in healthy subjects 20-80 years old.] Rev Med Chil. 2010; 138: 1124-30. 


\section{Chapter 3}

23. Ben Saad H, Prefaut C, Tabka Z, et al. 6-minute walk distance in healthy North Africans older than 40 years: influence of parity. Respir Med. 2009; 103: 74-84.

24. Dourado VZ, Vidotto MC and Guerra RL. Reference equations for the performance of healthy adults on field walking tests. J Bras Pneumol. 2011; 37: 607-14.

25. Camarri B, Eastwood PR, Cecins NM, Thompson PJ and Jenkins S. Six minute walk distance in healthy subjects aged 55-75 years. Respir Med. 2006; 100: 658-65.

26. Troosters T, Gosselink R and Decramer M. Six minute walking distance in healthy elderly subjects. Eur Respir J. 1999; 14: 270-4.

27. Hill K, Wickerson LM, Woon LJ, et al. The 6-min walk test: responses in healthy Canadians aged 45 to 85 years. Appl Physiol Nutr Metab 2011; 36: 643-9.

28. Gibbons WJ, Fruchter N, Sloan S and Levy RD. Reference values for a multiple repetition 6-minute walk test in healthy adults older than 20 years. J Cardiopulm Rehabil. 2001; 21: 87-93.

29. Jenkins S, Cecins N, Camarri B, Williams C, Thompson P and Eastwood P. Regression equations to predict 6-minute walk distance in middle-aged and elderly adults. Physiother Theory Pract. 2009; 25: 516-22.

30. Capodaglio P, De Souza SA, Parisio C, et al. Reference values for the 6-Min Walking Test in obese subjects. Disabil Rehabil 2013; 35: 1199-203.

31. Lee WTN, Peacock AJ and Johnson MK. The role of per cent predicted 6-min walk distance in pulmonary arterial hypertension. Eur Respir J. 2010; 36: 1294-301.

32. Dourado VZ. [Reference Equations for the 6-Minute Walk Test in Healthy Individuals.]. Arq Bras Cardiol. 2011.

33. Vaish H, Ahmed F, Singla R and Shukla DK. Reference equation for the 6-minute walk test in healthy North Indian adult males. Int J Tuberc Lung Dis. 2013; 17: 698-703.

34. Oliveira AC, Rodrigues CC, Rolim DS, et al. Six-minute walk test in healthy children: is the leg length important? Pediatr Pulmonol. 2013; 48: 921-6.

35. Gosselink R, Troosters T and Decramer M. Peripheral muscle weakness contributes to exercise limitation in COPD. Am J Respir Crit Care Med. 1996; 153: 976-80.

36. Dourado VZ, Antunes LC, Tanni SE, de Paiva SA, Padovani CR and Godoy I. Relationship of upper-limb and thoracic muscle strength to 6-min walk distance in COPD patients. Chest. 2006; 129: 551-7.

37. Sciurba F, Criner GJ, Lee SM, et al. Six-minute walk distance in chronic obstructive pulmonary disease: reproducibility and effect of walking course layout and length. Am J Respir Crit Care Med. 2003; 167: 1522-7.

38. Spruit MA, Vanderhoven-Augustin I, Janssen PP and Wouters EF. Integration of pulmonary rehabilitation in COPD. Lancet. 2008; 371: 12-3.

39. Decramer M, Bartsch P, Pauwels R and Yernault JC. Management of COPD according to guidelines. A national survey among Belgian physicians. Monaldi Arch Chest Dis. 2003; 59: 62-80.

40. Hernandes NA, Wouters EF, Meijer K, Annegarn J, Pitta F and Spruit MA. Reproducibility of 6-minute walking test in patients with COPD. Eur Respir J. 2011; 38: 261-7.

41. Kulkarni HS, Chandra D and Sciurba FC. Increase in walk distance is not enough evidence to add a walk to the 6-minute walk test. Eur Respir J. 2011; 38: 1239-40

42. Karvonen MJ, Kentala $E$ and Mustala O. The effects of training on heart rate; a longitudinal study. Ann Med Exp Biol Fenn. 1957; 35: 307-15.

43. Annegarn J, Spruit MA, Savelberg HH, et al. Differences in walking pattern during 6-min walk test between patients with COPD and healthy subjects. PLoS One. 2012; 7: e37329.

44. Sciurba F, Criner GJ, Lee SM, et al. Six-minute walk distance in chronic obstructive pulmonary disease: reproducibility and effect of walking course layout and length. Am J Resp Crit Care. 2003; 167: 1522-7.

45. Stevens D, Elpern E, Sharma K, Szidon P, Ankin M and Kesten S. Comparison of hallway and treadmill sixminute walk tests. Am J Respir Crit Care Med. 1999; 160: 1540-3. 


\section{SUPPLEMENTAL MATERIAL}

Table S1. Reference equations of the six-minute walk test in adults

\begin{tabular}{|c|c|c|}
\hline Studies & ist of Equations & $R^{2}$ \\
\hline lio & $\begin{array}{l}+0^{\lambda}: 6 \mathrm{MWD}_{\mathrm{m}}=894.2177-\left(2.0700 \times \text { age }_{\mathrm{yrs}}\right)-\left(51.4489 \times \text { gender }_{\text {men }=0 ; \text { women }=1}\right)-5.1663 \times \\
\mathrm{BMI} \mathrm{kg}^{2} \\
\text { * Reference equation for Obese subjects }\end{array}$ & .48 \\
\hline amanathan & 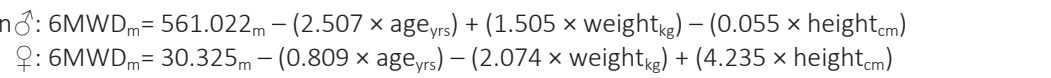 & $\begin{array}{l}0.29 \\
0.27\end{array}$ \\
\hline $\begin{array}{l}\text { Britto } \\
\text { et al. }\end{array}$ & 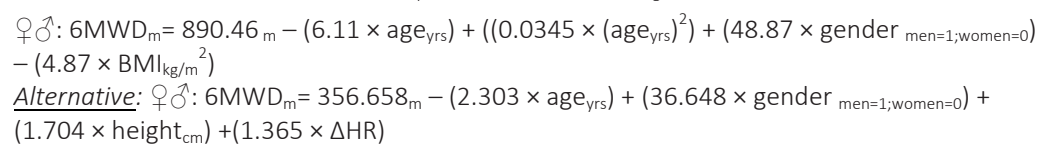 & 0.46 \\
\hline $\begin{array}{l}\text { Hill } \\
\text { et al. }{ }^{17}\end{array}$ & +ô: $6 \mathrm{MWD}_{\mathrm{m}}=970.7+\left(-5.5 \times\right.$ age $\left._{\mathrm{yrs}}\right)+\left(56.3 \times\right.$ gender $\left._{\text {men=1; women }=0}\right)$ & 0.45 \\
\hline & $\begin{array}{l}\text { o: } 6 \mathrm{MWD}_{\mathrm{m}}=361_{\mathrm{m}}-\left(\text { age }_{\mathrm{yrs}} \times 4\right)+\left(\text { height }_{\mathrm{cm}} \times 2\right)+\left(\mathrm{HR}_{\max } / \mathrm{HR}_{\max \% \text { pred }} \times 3\right)-\left(\text { weight }_{\mathrm{kg}} \times 1.5\right) \\
\text { +: } 6 \mathrm{MWD}_{\mathrm{m}}=361_{\mathrm{m}}-\left(\text { age }_{\mathrm{yrs}} \times 4\right)+\left(\text { height }_{\mathrm{cm}} \times 2\right)+\left(\mathrm{HR}_{\max } / \mathrm{HR}_{\max \% \mathrm{pred}} \times 3\right)-\left(\text { weight }_{\mathrm{kg}} \times 1.5\right)-30\end{array}$ & 0.38 \\
\hline & 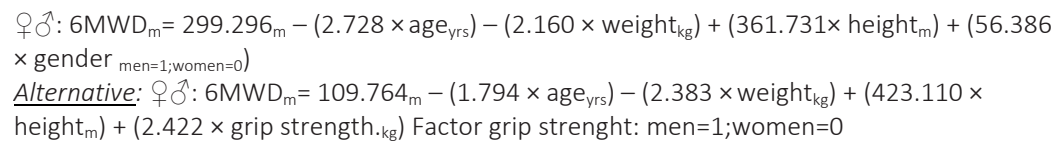 & \\
\hline & qô: $6 \mathrm{MWD} \mathrm{D}_{\mathrm{m}}=511_{\mathrm{m}}+\left(\left(\text { height }_{\mathrm{cm}}\right)^{2} \times 0.006\right.$ & 0.55 \\
\hline & $\begin{array}{l}\text { 1: } 6 \mathrm{MWD}_{\mathrm{m}}=530_{\mathrm{m}}-\left(3.31 \times \text { age }_{\mathrm{yrs}}\right)+\left(2.36 \times \text { height }_{\mathrm{cm}}\right)-(1 \\
\text { क: } 6 \mathrm{MWD}_{\mathrm{m}}=457_{\mathrm{m}}-\left(3.46 \times \text { age }_{\mathrm{yrs}}\right)+\left(2.61 \times \text { height }_{\mathrm{cm}}\right)-(1\end{array}$ & $\begin{array}{l}0.55 \\
0.63\end{array}$ \\
\hline & $+\partial^{\lambda}: 6 \mathrm{MWD}_{\mathrm{m}}=\left(2.81 \times\right.$ height $\left._{\mathrm{cm}}\right)+\left(0.79 \times\right.$ age $\left._{\mathrm{yrs}}\right)-28.5_{\mathrm{m}}$ & 0.25 \\
\hline $\mathrm{Be}$ & $\begin{array}{l}+\widehat{O}: 6 \mathrm{MWD}_{\mathrm{m}}=720.50_{\mathrm{m}}-\left(160 \times \text { gender }_{\text {men }=0 ; \text { women }=1}\right)-\left(5.14 \times \text { age }_{\mathrm{yrs}}\right)-\left(2.23 \times \text { weight }_{\mathrm{kg}}\right)+ \\
\left(2.72 \times \text { height }_{\mathrm{cm}}\right) \\
\text { LLN: Of the predicted } 6 \mathrm{MWD} \text { values a less of }-89 \mathrm{~m} \text { can be considered as abnormal }\end{array}$ & 0.77 \\
\hline רa & 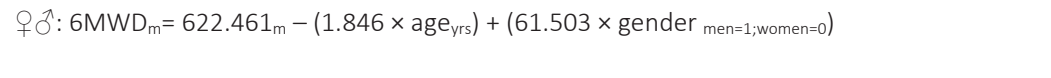 & 0.30 \\
\hline $\begin{array}{l}\text { Jenkins } \\
\text { et al. }{ }^{9}\end{array}$ & $\begin{array}{l}\text { đ: } 6 \mathrm{MWD}_{\mathrm{m}}=867-\left(5.71 \times \text { age }_{\mathrm{yrs}}\right)+\left(1.03 \times \text { height }_{\mathrm{cm}}\right) \\
\text { क: } 6 \mathrm{MWD}_{\mathrm{m}}=525_{\mathrm{m}}-\left(2.86 \times \text { age }_{\mathrm{yrs}}\right)+\left(2.71 \times \text { height }_{\mathrm{cm}}\right)-\left(6.22 \times \mathrm{BMI}_{\mathrm{kg} / \mathrm{m}}{ }^{2}\right)\end{array}$ & $\begin{array}{l}0.40 \\
0.43\end{array}$ \\
\hline $\begin{array}{l}\text { Masmoudi } \\
\text { et al. }\end{array}$ & 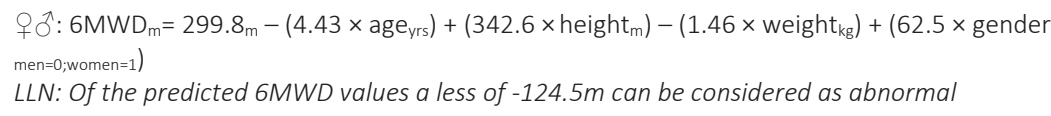 & \\
\hline arri & 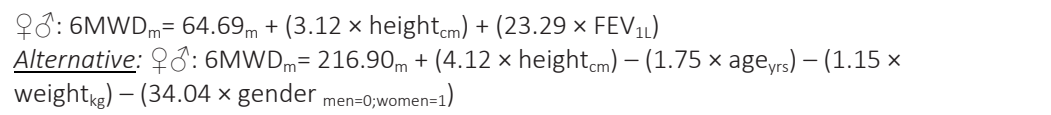 & 0.36 \\
\hline & +o : $\mathrm{MWD}_{\mathrm{m}}=518.853_{\mathrm{m}}+\left(1.25 \times\right.$ height $\left._{\mathrm{cm}}\right)-\left(2.816 \times\right.$ age $\left._{\mathrm{yrs}}\right)-\left(39.07 \times\right.$ gender $\left._{\text {men }=0 ; \text { women }=1}\right)$ & 0.42 \\
\hline $\begin{array}{l}\text { Poh } \\
\text { et al. }\end{array}$ & $\begin{array}{l}\text { +o }: 6 \mathrm{MWD}_{\mathrm{m}}=\left(5.50 \times \mathrm{HR}_{\max } / \mathrm{HR}_{\max \% \mathrm{pred}}\right)+\left(6.94 \times \text { height }_{\mathrm{cm}}\right)-\left(4.49 \times \text { age }_{\mathrm{yrs}}\right)-\left(3.51 \times \text { weight }_{\mathrm{kg}}\right) \\
-473.27_{\mathrm{m}}\end{array}$ & ) \\
\hline $\begin{array}{l}\text { Enright } \\
\text { et al. }{ }^{4}\end{array}$ & $\begin{array}{l}\text { J: } 6 \mathrm{MWD}_{\mathrm{m}}=510_{\mathrm{m}}+\left(2.2 \times \text { height }_{\mathrm{cm}}\right)-\left(0.93 \times \text { weight }_{\mathrm{kg}}\right)-\left(5.3 \times \text { age }_{\mathrm{yrs}}\right) \\
\text { क: } 6 \mathrm{MWD} \mathrm{m}=493_{\mathrm{m}}+\left(2.2 \times \text { height }_{\mathrm{cm}}\right)-\left(0.93 \times \text { weight }_{\mathrm{kg}}\right)-\left(5.3 \times \text { age }_{\mathrm{yrs}}\right) \\
\text { LLN: Of the predicted } 6 \mathrm{MWD} \text { values a less of }-100 \mathrm{~m} \text { for men \& women can be considered as } \\
\text { abnormal }\end{array}$ & 0.20 \\
\hline
\end{tabular}




\section{Chapter 3}

\begin{tabular}{|c|c|c|}
\hline Studies & List of Equations & $R^{2}$ \\
\hline $\begin{array}{l}\text { Gibbons } \\
\text { et al. }{ }^{3}\end{array}$ & qô: $6 \mathrm{MWD}_{\mathrm{m}}=868.8_{\mathrm{m}}-\left(\right.$ age $\left._{\mathrm{yrs}} \times 2.99\right)-\left(\right.$ gender $\left._{\text {men=0;women }=1} \times 74.7\right)$ & 0.41 \\
\hline $\begin{array}{l}\text { Troosters } \\
\text { et al. }{ }^{2}\end{array}$ & $\begin{array}{l}\text { +o : } 6 \mathrm{MWD}_{\mathrm{m}}=218_{\mathrm{m}}+\left(5.14 \times \text { height }_{\mathrm{cm}}-5.32 \times \text { age }_{\mathrm{yrs}}\right)-\left(1.80 \times \text { weight }_{\mathrm{kg}}\right)+(51.31 \times \text { gender } \\
\text { men=1; women=0); } \\
\text { LLN: Of the predicted } 6 \mathrm{MWD} \text { values }<82 \% \text { can be considered as abnormal }\end{array}$ & 0.66 \\
\hline $\begin{array}{l}\text { Enright \& } \\
\text { Sherrill }^{1}\end{array}$ & $\begin{array}{l}\text { J: } 6 \mathrm{MWD}_{\mathrm{m}}=\left(7.57 \times \text { height }_{\mathrm{cm}}\right)-\left(5.02 \times \text { age }_{\mathrm{yrs}}\right)-\left(1.76 \times \text { weight }_{\mathrm{kg}}\right)-309_{\mathrm{m}} \\
\text { क: } 6 \mathrm{MWD}_{\mathrm{m}}=\left(2.11 \times \text { height }_{\mathrm{cm}}\right)-\left(2.29 \times \text { weight }_{\mathrm{kg}}\right)-\left(5.78 \times \mathrm{age}_{\mathrm{yrs}}\right)+667_{\mathrm{m}} \\
\text { Alternatives: } 01: 6 \mathrm{MWD}_{\mathrm{m}}=1.140_{\mathrm{m}}-\left(5.61 \times \mathrm{BMI}_{\mathrm{kg} / \mathrm{m}}{ }^{2}\right)-\left(6.94 \times \mathrm{age}_{\mathrm{yrs}}\right) \\
\text { क: } 6 \mathrm{MWD} \mathrm{D}_{\mathrm{m}}=1.017_{\mathrm{m}}-\left(6.24 \times \mathrm{BMI}_{\mathrm{kg} / \mathrm{m}}{ }^{2}\right)-\left(5.83 \times \text { age }_{\mathrm{yrs}}\right) \\
\text { LLN: Of the predicted } 6 \mathrm{MWD} \text { values a less of }-153 \mathrm{~m} \text { for men \& }-139 \mathrm{~m} \text { for women can be } \\
\text { considered as abnormal }\end{array}$ & $\begin{array}{l}0.42 \\
0.38 \\
0.42 \\
0.38\end{array}$ \\
\hline
\end{tabular}

List of 6MWT references equations derived from 6MWD reference values of healthy adults. 
Table S2. Technical and methodological information of studies that formulated 6MWT reference values

\begin{tabular}{|c|c|c|c|c|c|c|}
\hline Studies & Partic. (n) & Females (\%) & Age (Range) & Race & $6 \mathrm{MWD}, \mathrm{m}$ & Methodological notes \\
\hline $\begin{array}{l}\text { Capodaglio } \\
\text { et al. }{ }^{20 *}\end{array}$ & 323 & $194(60)$ & $37(20-60)$ & $\begin{array}{l}\text { Caucasian } \\
\text { Italians }\end{array}$ & $565 \pm 48$ & $\begin{array}{l}{ }^{*} \mathrm{BMI} \geq 30 \mathrm{~kg} / \mathrm{m}^{2}, \text { Equation } \\
\text { specific for obese subjects }\end{array}$ \\
\hline $\begin{array}{l}\text { Ramanatha } \\
\text { n et al. }{ }^{19}\end{array}$ & 125 & $67(54)$ & $46(25-80)$ & Indians & $495 \pm 84$ & $\begin{array}{l}\text { Convenience-based sample of } \\
\text { participants }\end{array}$ \\
\hline $\begin{array}{l}\text { Britto } \\
\text { et al. }\end{array}$ & 617 & $321(52)$ & $52(29-67)$ & Brazilians & $586 \pm 106$ & Not ramdomized sample \\
\hline $\begin{array}{l}\text { Hill } \\
\text { et al. }{ }^{17}\end{array}$ & 77 & $40(52)$ & $65(45-85)$ & Canadians & $640 \pm 99$ & $\begin{array}{l}\text { Participants from } 1 \text { specific } \\
\text { region }\end{array}$ \\
\hline $\begin{array}{l}\text { Casanova } \\
\text { et al. }^{16}\end{array}$ & 444 & $206(46)$ & $58(42-76)$ & Mixed & $571 \pm 90$ & Not ramdomized sample \\
\hline $\begin{array}{l}\text { Dourado } \\
\text { et al. }^{15}\end{array}$ & 90 & $50(56)$ & $60(40-70)$ & Brazilians & $572 \pm 83$ & $\begin{array}{l}\text { Convenience-based sample of } \\
\text { participants, Inclusion of Grip } \\
\text { Strength }\end{array}$ \\
\hline $\begin{array}{l}\text { Soares } \\
\text { et al. }^{14}\end{array}$ & 132 & $66(50)$ & $(20-80)$ & Brazilians & $\begin{array}{l}\text { All: } 552 \text { (Weighted) } \\
\text { o: } 566 \pm 87 \\
\text { ㅇ: } 538 \pm 95\end{array}$ & $\begin{array}{l}\text { Convenience-based sample of } \\
\text { participants }\end{array}$ \\
\hline $\begin{array}{l}\text { Osses } \\
\text { et al. }{ }^{13}\end{array}$ & 175 & $98(56)$ & $55(20-80)$ & Brazilians & $\begin{array}{l}\text { All: } 605 \text { (Weighted) } \\
\text { đ: } 644 \pm 84 \\
\text { : } 576 \pm 87\end{array}$ & \\
\hline $\begin{array}{l}\text { Alameri } \\
\text { et al. }\end{array}$ & 238 & $111(47)$ & $29(16-50)$ & Arabs & $\begin{array}{l}\text { All: } 409 \pm 51 \\
\text { o: } 429 \pm 47 \\
\text { o: } 386 \pm 45\end{array}$ & $\begin{array}{l}\text { "walk at your own pace", } \\
\text { HRmax pred. Males: } 44 \% \\
\text { Females: } 47 \%\end{array}$ \\
\hline $\begin{array}{l}\text { Ben Saad } \\
\text { et al. }{ }^{11}\end{array}$ & 229 & $125(55)$ & $56(45-59)$ & Tunisians & $\begin{array}{l}\text { All: } 624 \pm 111 \\
\text { đ: } 711 \pm 81 \\
\text { o: } 551 \pm 75\end{array}$ & $40 m$ corridor \\
\hline $\begin{array}{l}\text { Iwama } \\
\text { et al. }\end{array}$ & 134 & $73(55)$ & $33(13-84)$ & Brazilians & $\begin{array}{l}\text { All: } 583 \pm 83 \\
\text { ふ: } 622 \pm 80 \\
\text { : } 551 \pm 74\end{array}$ & $\begin{array}{l}\text { Only } 5 \text { participants were } \\
>65 y r s \text {, the } 2^{\text {nd }} 6 \mathrm{MWD} \text { was } \\
\text { used and not the best }\end{array}$ \\
\hline $\begin{array}{l}\text { Jenkins } \\
\text { et al. }{ }^{9}\end{array}$ & 109 & $61(56)$ & $62(45-85)$ & $\begin{array}{l}\text { Caucasian } \\
\text { Australian }\end{array}$ & $\begin{array}{l}\text { All: } 660 \text { (Weighted) } \\
\text { } 1: 682 \pm 73 \\
\text { ㅇ: } 643 \pm 70\end{array}$ & $45 \mathrm{~m}$ corridor \\
\hline $\begin{array}{l}\text { Masmoudi } \\
\text { et al. }\end{array}$ & 155 & $75(48)$ & $(40-80)$ & Tunisians & - & - \\
\hline $\begin{array}{l}\text { Camarri } \\
\text { et al. }\end{array}$ & 70 & $37(53)$ & $65(55-75)$ & $\begin{array}{l}\text { Caucasian } \\
\text { Australian }\end{array}$ & $659 \pm 62$ & $\begin{array}{l}\text { HRmax pred. } 81 \% \text {, } \\
45 \text { m corridor }\end{array}$ \\
\hline $\begin{array}{l}\text { Chetta } \\
\text { et al. }^{6}\end{array}$ & 102 & $54(53)$ & $34(20-50)$ & $\begin{array}{l}\text { Caucasian } \\
\text { Italian }\end{array}$ & $\begin{array}{l}\text { All: } 614 \text { (Weighted) } \\
\hat{\jmath}: 638 \pm 44 \\
\text { : } 593 \pm 57\end{array}$ & $\begin{array}{l}\text { Relatively young group of } \\
\text { participants }\end{array}$ \\
\hline $\begin{array}{l}\text { Poh } \\
\text { et al. }\end{array}$ & 35 & $19(54)$ & $61(45-85)$ & $\begin{array}{l}\text { Chinese } \\
\text { Singapor. }\end{array}$ & $560 \pm 105$ & $45 \mathrm{~m}$ corridor \\
\hline $\begin{array}{l}\text { Enright } \\
\text { et al. }{ }^{4}\end{array}$ & 2117 & $1270(60)$ & $77(\geq 70)$ & Mixed & $\begin{array}{l}\text { All: } 334 \\
\text { ग: } 362 \\
\text { : } 332\end{array}$ & $\begin{array}{l}\text { Participants with risk factors } \\
\text { associated with cardiovascular } \\
\text { disease }\end{array}$ \\
\hline $\begin{array}{l}\text { Gibbons } \\
\text { et al. }{ }^{3}\end{array}$ & 79 & $38(48)$ & $45(20-80)$ & Canadian & $698 \pm 96$ & $\begin{array}{l}20 \mathrm{~m} \text { corridor, Equation based } \\
\text { on the maximum of four } 6 \mathrm{MWT}\end{array}$ \\
\hline $\begin{array}{l}\text { Troosters } \\
\text { et al. }{ }^{2}\end{array}$ & 51 & $22(43)$ & $65(50-85)$ & $\begin{array}{l}\text { Caucasian } \\
\text { Belgian }\end{array}$ & $631 \pm 93$ & HRmax pred. $77 \%$ \\
\hline $\begin{array}{l}\text { Enright \& } \\
\text { Sherrill }^{1}\end{array}$ & 290 & $173(60)$ & $61(40-80)$ & $\begin{array}{l}\text { Caucasian } \\
\text { American }\end{array}$ & $\begin{array}{l}\text { All: } 527 \text { (Weighted) } \\
\text { đ: } 576 \\
\text { o: } 494\end{array}$ & $\begin{array}{l}\text { No practice test, } \\
\text { HRmax pred. }<65 \%\end{array}$ \\
\hline
\end{tabular}




\section{Chapter 3}

Technical and methodological differences of studies that formulated 6MWT reference values derived from healthy adults.

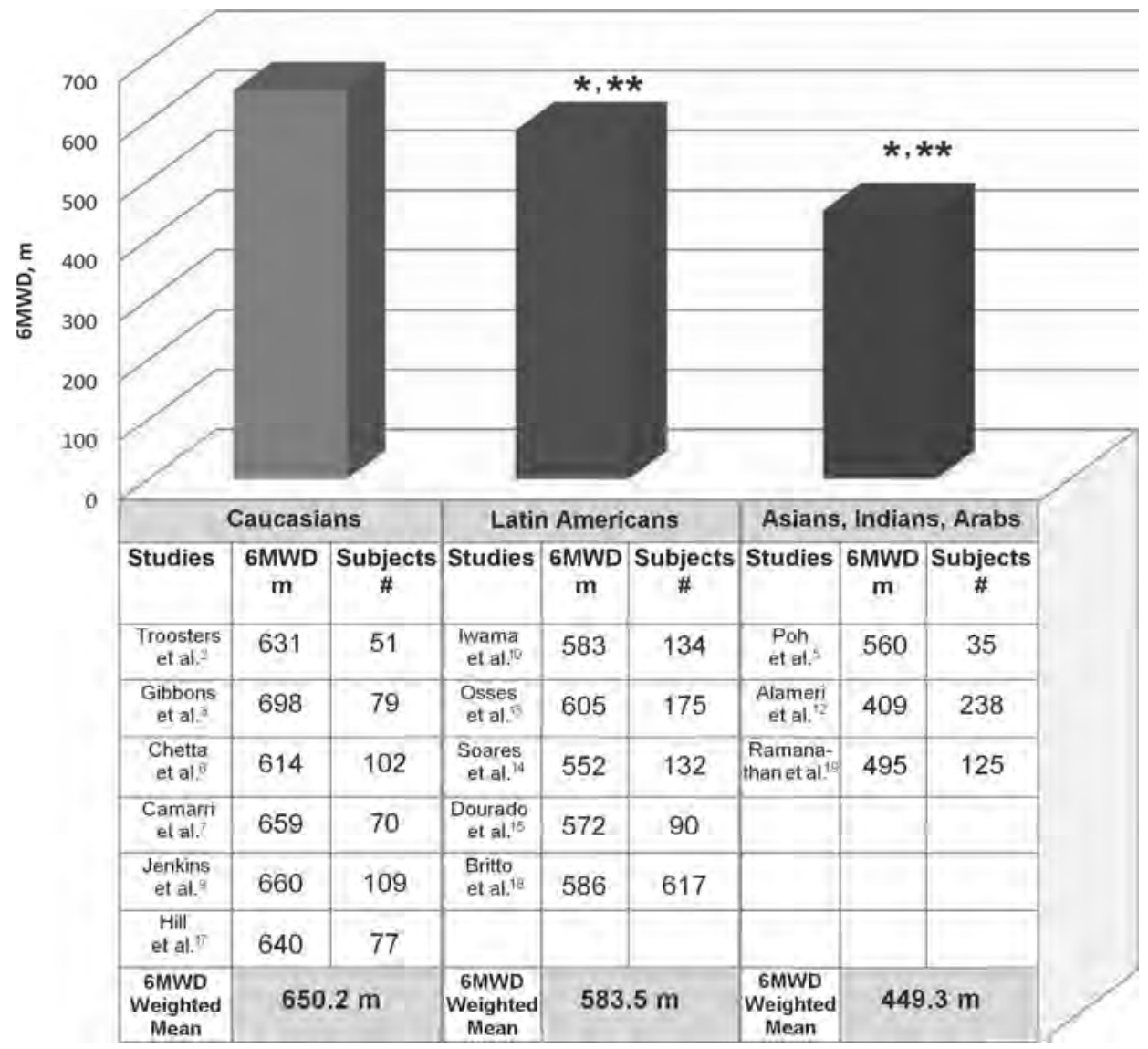

Figure S1. Performance quartile comparisons of CPET

Weighted means of the 6MWD of several healthy populations categorized by race. Asterisks denote significant differences between the first group $\left({ }^{*}\right)$ and between the second group $\left({ }^{* *}\right)(p<0.05)$. 


\section{REFERENCES}

1. Enright PL and Sherrill DL. Reference equations for the six-minute walk in healthy adults. Am J Respir Crit Care Med. 1998; 158: 1384-7.

2. Troosters T, Gosselink R and Decramer M. Six minute walking distance in healthy elderly subjects. Eur Respir J. 1999; 14: 270-4.

3. Gibbons WJ, Fruchter N, Sloan S and Levy RD. Reference values for a multiple repetition 6-minute walk test in healthy adults older than 20 years. J Cardiopulm Rehabil. 2001; 21: 87-93.

4. Enright PL, McBurnie MA, Bittner $\mathrm{V}$, et al. The 6-min walk test: a quick measure of functional status in elderly adults. Chest. 2003; 123: 387-98.

5. Poh H, Eastwood PR, Cecins NM, Ho KT and Jenkins SC. Six-minute walk distance in healthy Singaporean adults cannot be predicted using reference equations derived from Caucasian populations. Respirology. 2006; 11: 211-6.

6. Chetta A, Zanini A, Pisi G, et al. Reference values for the 6-min walk test in healthy subjects 20-50 years old. Respir Med. 2006; 100: 1573-8.

7. Camarri B, Eastwood PR, Cecins NM, Thompson PJ and Jenkins S. Six minute walk distance in healthy subjects aged 55-75 years. Respir Med. 2006; 100: 658-65.

8. Masmoudi K, Aouicha MS, Fki H, Dammak J and Zouari N. [The six minute walk test: which predictive values to apply for Tunisian subjects aged between 40 and 80 years?]. Tunis Med. 2008; 86: 20-6.

9. Jenkins S, Cecins N, Camarri B, Williams C, Thompson P and Eastwood P. Regression equations to predict 6-minute walk distance in middle-aged and elderly adults. Physiother Theory Pract. 2009; 25: 516-22.

10. Iwama AM, Andrade GN, Shima P, Tanni SE, Godoy I and Dourado VZ. The six-minute walk test and body weight-walk distance product in healthy Brazilian subjects. Braz J Med Biol Res. 2009; 42: 1080-5.

11. Ben Saad H, Prefaut C, Tabka Z, et al. 6-minute walk distance in healthy North Africans older than 40 years: influence of parity. Respir Med. 2009; 103: 74-84.

12. Alameri H, Al-Majed S and Al-Howaikan A. Six-min walk test in a healthy adult Arab population. Respir Med. 2009; 103: 1041-6.

13. Osses AR, Yanez VJ, Barria PP, et al. [Reference values for the 6-minutes walking test in healthy subjects 20-80 years old]. Rev Med Chil. 2010; 138: 1124-30.

14. Soares MR and Pereira CA. Six-minute walk test: reference values for healthy adults in Brazil. J Bras Pneumol. 2011; 37: 576-83.

15. Dourado VZ, Vidotto MC and Guerra RL. Reference equations for the performance of healthy adults on field walking tests. J Bras Pneumol. 2011; 37: 607-14.

16. Casanova C, Celli BR, Barria P, et al. The 6-min walk distance in healthy subjects: reference standards from seven countries. Eur Respir J. 2011; 37: 150-6.

17. Hill K, Wickerson LM, Woon LJ, et al. The 6-min walk test: responses in healthy Canadians aged 45 to 85 years. Appl Physiol Nutr Metab. 2011; 36: 643-9.

18. Britto RR, Probst VS, de Andrade AF, et al. Reference equations for the six-minute walk distance based on a Brazilian multicenter study. Braz J Phys Ther. 2013; 17: 556-63.

19. Palaniappan Ramanathan $R$ and Chandrasekaran B. Reference equations for 6 -min walk test in healthy Indian subjects (25-80 years). Lung India. 2014; 31: 35-8.

20. Capodaglio P, De Souza SA, Parisio C, et al. Reference values for the 6-Min Walking Test in obese subjects. Disabil Rehabil. 2013; 35: 1199-203. 



\section{Chapter}

\section{Prognostic value of variables derived from the six-minute walk test in patients with COPD: Results from the ECLIPSE study}

Vasileios Andrianopoulos, Emiel F.M. Wouters, Victor M. Pinto-Plata, Lowie E.G.W. Vanfleteren, Per S. Bakke, Frits M.E. Franssen, Alvar Agusti, William MacNee, Stephen I. Rennard, Ruth Tal Singer, Ioannis Vogiatzis, Jørgen

Vestbo, Bartolome R. Celli, Martijn A. Spruit 


\section{Chapter 4}

\section{ABSTRACT}

\section{Background}

In addition to the six-min walk distance (6MWD), other six-min walk test (6MWT) derived variables, such as mean walk-speed (6MWSpeed), 6-min walk-work (6MWW), distance-saturation product (DSP), exercise-induced oxygen desaturation (EID), and unintended stops may be useful for the prediction of mortality and hospitalization in patients with chronic obstructive pulmonary disease (COPD). We studied the association between 6MWT-derived variables and mortality as well as hospitalization in COPD patients and compared it with the BODE index.

\section{Methods}

A three-year prospective study (ECLIPSE) to evaluate the prognostic value of 6MWTderived variables in 2010 COPD patients. Cox's proportional-hazard regressions were performed to estimate 3-year mortality and hospitalization.

\section{Results}

During the follow-up, 193 subjects died and 622 were hospitalized. An adjusted Cox's regression model of hazard ratio [HR] for impaired 6MWT-derived variables was significant referring to: mortality (6MWD $\leq 334 \mathrm{~m}$ [2.30], 6MWSpeed $\leq 0.9 \mathrm{~m} / \mathrm{sec}$ [2.15], $6 \mathrm{MWW} \leq 20000 \mathrm{~m} \mathrm{~kg}$ [2.17], DSP $\leq 290 \mathrm{~m} \%$ [2.70], EID $\leq 88 \%$ [1.75], unintended stops [1.99]; and hospitalization (6MWW $\leq 27000 \mathrm{~m} \cdot \mathrm{kg}$ [1.23], EID $\leq 88 \%$ [1.25], BODE index $\geq 3$ points [1.40]; all $p \leq 0.05)$.

\section{Conclusions}

The 6MWT-derived variables have an additional predictive value of mortality in patients with COPD. The 6MWW, EID and the BODE index refine the prognosis of hospitalization. 


\section{INTRODUCTION}

The six-minute walk test (6MWT) is a reliable, widely-used test to assess functional exercise performance in patients with chronic obstructive pulmonary disease (COPD). ${ }^{1-3}$ Its primary readout is the distance (meters) walked in six minutes (6MWD). A 6MWD $<350 \mathrm{~m}$ predicts an increased risk of hospitalization and mortality rates in COPD. ${ }^{4,5}$

The 6MWT is generally well tolerated, but some patients with COPD are unable to walk at a continuous pace for six minutes and need to stop to rest. Those unintended stops due to exercise-induced symptoms such as dyspnea and leg discomfort may even lead to test discontinuation. ${ }^{6,7}$ Obviously, unintended stops significantly affect 6MWD, and, in turn, may be the underlying reason that a poor 6MWD is associated with mortality in patients with COPD. Indeed, unintended stops during corridor walking are associated with higher rates of mortality in older adults and should be evaluated..$^{8-11}$ Therefore, we hypothesized that unintended stop(s) during the 6MWT may provide an additional prognostic information besides the 6MWD of patients with COPD. In addition, other 6MWT-derived outcomes, such as the average walking speed (6MWSpeed, m/sec of actual walking) ${ }^{5,9,10}$, the 6-min walk work (6MWW, m kg; defined as the product of 6MWD in meters and body weight in kilograms) ${ }^{12,13}$, the distancesaturation product (DSP, in meters\% defined as the product of the 6MWD in meters and the lowest $\left.\mathrm{SpO}_{2} \%\right)^{14}$, and the exercise-induced oxygen desaturation (EID; defined as a nadir $\mathrm{SpO}_{2} \leq 88 \%$ in the $\left.6 \mathrm{MWT}\right)^{15}$ might also possess significant prognostic value in identifying patients at high risk for mortality and hospitalization in COPD.

To date, little is known about the prognostic value of the above mentioned 6MWTderived variables in patients with COPD. Therefore, we sought to determine the prognostic value of the 6MWT-derived variables exploring further data from the ECLIPSE study. ${ }^{16}$

We used the well validated BODE index ${ }^{17,18}$, calculated from body mass index (B), degree of airflow obstruction as expressed by $\mathrm{FEV}_{1}(\mathrm{O})$, dyspnea with the modified medical research council (D) and exercise (E) measured with the 6MWD, as a comparator.

\section{METHODS}

\section{Design and participants}

The ECLIPSE study (ClinicalTrials.gov identifier: NCT00292552; GSK study code SCO104960) was a 3-year non-interventional longitudinal prospective study. The inclusion and exclusion criteria of the ECLIPSE study have been described elsewhere. ${ }^{16}$ In brief, individuals (age: 40-75 years) were recruited to the ECLIPSE if they had a smoking 


\section{Chapter 4}

history of $\geq 10$ pack-years and a diagnosis of COPD. ${ }^{19}$ The ECLIPSE study was carried out in accordance with the Declaration of Helsinki and good clinical practice guidelines, and approved by the ethics committees of participating centers. The Institutional Review Boards of all participating institutions approved the study and all participants signed their informed consent.

\section{Subject characterization}

Demographic and physiological characteristics, level of dyspnea (using the modified Medical Research Council (mMRC) dyspnea scale) and measurements of lung function (post-bronchodilator spirometry and lung volumes), amount of emphysema (low-dose volumetric computed tomography [CT scan] $120 \mathrm{kV}$ peak, $40 \mathrm{~mA}$ and 1.00 or $1.25-\mathrm{mm}$ slice thickness), and exercise data (6MWT; see below) were used for analyses. Fat mass and fat free mass were determined using bioelectrical impedance (Bodystat 1500). Fat free mass index (FFMI, $\mathrm{kg} / \mathrm{m}^{2}$ ) was calculated as the mass $(\mathrm{kg})$ divided by the squared height $\left(\mathrm{m}^{2}\right)$ and the body mass index $\left(\mathrm{BMI}, \mathrm{kg} / \mathrm{m}^{2}\right)$ was calculated as weight $(\mathrm{kg})$ divided by squared height $\left(\mathrm{m}^{2}\right)$.

\section{Six-minute walk test}

The 6-min walk test was performed according to the 2002 ATS guidelines. ${ }^{20}$ Briefly, participants were asked to walk indoors in a flat, straight, $30 \mathrm{~m}$ walking course supervised by a well-trained researcher. A practice 6MWT was not undertaken. Subjects were encouraged using standard methodology every minute of the 6MWT. ${ }^{21}$ They were allowed to stop and rest during the test, but were instructed to resume walking as soon as possible. Rest and post-exercise $\mathrm{SpO}_{2}$ were measured using a pulse oxymeter with a finger probe. A modified Borg scale was used to quantify the level of dyspnea and fatigue perceived by subjects at the beginning and end of the test. The BODE index was calculated using the algorithm developed by Celli and colleagues. ${ }^{17}$ We report that $6 \mathrm{MWT}$-data from the ECLIPSE study have been published before. Specifically, the association between baseline 6MWD and outcomes; and between changes in 6MWD and outcomes has been published. 22,23 The current study is complementary, as the $6 \mathrm{MWT}$-derived variables and their associations with survival and hospitalization are novel.

Several 6MWT derived variables were used for analyses. The 6MWD was recorded by measuring the actual walking distance in meters during the 6MWT; 6MWSpeed was calculated by dividing the 6MWD by the total walking time $(6 \mathrm{MWSpeed}, \mathrm{m} / \mathrm{sec}=$ 6MWD, m/walking time, sec). ${ }^{5,9}$ For example, in a 6MWD of $400 \mathrm{~m}$ during which patient have unintended stop(s) of a total duration of 30 seconds, the 6MWSpeed would be 1.2 $\mathrm{m} / \mathrm{sec}$ (e.g. $400 \mathrm{~m} / 330 \mathrm{sec}$ ). 6MWW was estimated by the product of body weight and 
walking distance $(6 \mathrm{MWW}, \mathrm{m} \mathrm{kg}=6 \mathrm{MWD}, \mathrm{m} \times$ body weight, $\mathrm{kg}) .{ }^{12,13}$ DSP was calculated as the product of the final distance walked in meters and the post-exercise $\mathrm{SpO}_{2} \%$ (DSP, $\mathrm{m} \%=6 \mathrm{MWD}, \mathrm{m} \times \mathrm{SpO}_{2} \%$ post $\left./ 100\right) .{ }^{14}$ EID was defined as the post-exercise $\mathrm{SpO}_{2} \leq 88 \% .{ }^{15}$ Unintended stops were defined as a (temporary) discontinuation of the 6MWT.

\section{Statistical analyses}

Subjects were classified as: (1) "Completers" or "Stoppers" according to their ability to complete the 6MWT; (2) "Hospitalized" or "Non-hospitalized" according to $\geq 1$ exacerbations requiring hospitalization during follow up (exacerbation-related hospitalization); and (3) "Survivors" or "Non-survivors" according to their vital status at the end of 3-year follow-up.

Results are shown as mean (standard deviation) or proportion (\%). The statistical significance of between-group differences was assessed using analysis of variance, paired T-tests and Chi-square test, as appropriate. Multiple comparisons were testing with one-way Anova followed by Bonferroni's post-hoc tests. Receiver operating characteristics (ROC) curves were used to determine threshold values with the best sensitivity and specificity to predict 3-year survival and hospitalization. The Area Under the curve $(A \cup C)$ was calculated by the trapezoidal rule and the confidence intervals of the AUC was computed by the method of DeLong. ${ }^{24}$ Kaplan-Meier curves based on unadjusted and adjusted analyses with Cox's proportional-hazard regression estimated the 3-year survival probability and the hospitalization-free probability based on patients' characteristics. Cox's proportional-hazard regression model was adjusted for age, sex, FEV $1, F E V_{1} / F V C$ ratio (Tiffeneau index), SGRQ, emphysema and smoking. Estimated hazard ratio (HR) and 95\% confidence intervals $(\mathrm{Cl})$ were calculated. Logminus-log plots confirmed the validity of the proportionality of hazards assumption over time. A two-sided level of significance was set at $P<0.05$. Statistical analyses were carried out using MedCalc v.12 and SPSS v.19.0.

\section{RESULTS}

\section{Study characteristics}

A total of 2010 COPD patients with complete data was included in the study. As shown in Table 1, subjects had normal BMI, moderate to severe airflow limitation (56\% GOLD grade III or IV) and mild to severe emphysema on CT (34\% had severe to very severe emphysema). The $53 \%$ of subjects had dyspnea (mMRC scale $\geq 2$ ), $48 \%$ severely impaired health status (SGRQ Total Score $\geq 50$ points), and $27 \%$ symptoms of depression (CES-D scale $\geq 16$ points; Table 1). On average, subjects walked $372 \pm 122 \mathrm{~m}$; $6 \mathrm{MWSpeed}$ was 1.0 


\section{Chapter 4}

$\pm 0.3 \mathrm{~m} / \mathrm{s} ; 6 \mathrm{MWW}$ was $28271 \pm 11232 \mathrm{~m} \mathrm{~kg}$; and DSP was $341 \pm 117 \mathrm{~m} \%$. The $6 \mathrm{MWD}$ was $<350 \mathrm{~m}$ in $41 \%$ of the cohort, $23 \%$ exhibited EID, and $4 \%$ had unintended stops during the 6MWT. The BODE index in the entire cohort was $3.1 \pm 2.1$ points (Table 1 ).

Table 1. Baseline characteristics of COPD patients categorized by mortality and hospitalization during a 3-year follow-up

\begin{tabular}{|c|c|c|c|c|c|c|c|}
\hline Characteristics & $\begin{array}{l}\text { All } \\
(n=2010)\end{array}$ & $\begin{array}{l}\text { Survivors } \\
(n=1817,90 \%)\end{array}$ & $\begin{array}{l}\text { Non- } \\
\text { survivors } \\
(n=193,10 \%)\end{array}$ & P Value & $\begin{array}{l}\text { Non- } \\
\text { hospitalized } \\
(n=1388,69 \%)\end{array}$ & $\begin{array}{l}\text { Hospitalized } \\
(n=622,31 \%)\end{array}$ & P Value \\
\hline Women, n, (\%) & $698(35)$ & $641(35)$ & $57(30)$ & & $478(34)$ & $220(35)$ & \\
\hline Age, years & $63.4 \pm 7.1$ & $63.1 \pm 7.1$ & $66.1 \pm 6.5$ & $<0.001$ & $63.1 \pm 7.2$ & $64.1 \pm 6.7$ & 0.002 \\
\hline BMI, kg/m² & $26.5 \pm 5.6$ & $26.5 \pm 5.5$ & $26.2 \pm 6.3$ & NS & $26.7 \pm 5.6$ & $26.0 \pm 5.5$ & 0.007 \\
\hline$<21$ & $295(15)$ & $254(14)$ & $41(21)$ & & $185(13)$ & $110(18)$ & \\
\hline $21-30$ & $1273(63)$ & 1170 (64) & $103(54)$ & & $891(64)$ & $382(61)$ & \\
\hline$\geq 30$ & $442(22)$ & $393(22)$ & $49(25)$ & $N S^{\#}$ & $312(23)$ & $130(21)$ & $N S^{\#}$ \\
\hline FFMI, $\mathrm{kg} / \mathrm{m}^{2}$ & $17.8 \pm 3.4$ & $17.8 \pm 3.3$ & $17.7 \pm 4.1$ & NS & $18.0 \pm 3.4$ & $17.3 \pm 3.5$ & $<0.001$ \\
\hline $\mathrm{FEV}_{1}, \%$ pred. & $48.5 \pm 15.5$ & $49.2 \pm 15.5$ & $42.3 \pm 15.0$ & $<0.001$ & $51.3 \pm 15.3$ & $42.4 \pm 14.4$ & $<0.001$ \\
\hline FVC, \% pred. & $80.0 \pm 19.7$ & $80.6 \pm 19.7$ & $73.8 \pm 18.4$ & $<0.001$ & $81.8 \pm 19.6$ & $75.8 \pm 19.3$ & $<0.001$ \\
\hline $\mathrm{FEV}_{1} / \mathrm{FVC}, \%$ & $44.5 \pm 11.4$ & $44.8 \pm 11.4$ & $41.7 \pm 11.0$ & $<0.001$ & $46.1 \pm 11.4$ & $40.9 \pm 10.5$ & $<0.001$ \\
\hline \multicolumn{8}{|l|}{ GOLD Stage } \\
\hline Stage II & $891(44)$ & $835(46)$ & $56(29)$ & & $720(52)$ & $171(28)$ & \\
\hline Stage III & $862(43)$ & $767(42)$ & 95 (49) & & $542(39)$ & $320(51)$ & \\
\hline Stage IV & $257(13)$ & $215(12)$ & $42(22)$ & $<0.001^{\#}$ & $126(9)$ & $131(21)$ & $<0.001^{\#}$ \\
\hline \multicolumn{8}{|c|}{ Emphysema extent $^{\S}$} \\
\hline$<5 \%$ & $461(25)$ & $430(26)$ & $31(18)$ & & $372(29)$ & $89(16)$ & \\
\hline $5-25 \%$ & $397(22)$ & $368(22)$ & $29(17)$ & & $289(23)$ & $108(19)$ & \\
\hline $25-50 \%$ & 351 (19) & 316 (19) & $35(21)$ & & 238 (19) & $113(20)$ & \\
\hline$>50 \%$ & $618(34)$ & $543(33)$ & $75(44)$ & $<0.001^{\#}$ & $362(29)$ & $256(45)$ & $<0.001^{\#}$ \\
\hline LAA\%, $-950_{\mathrm{HU}}$ & $17.5 \pm 12.1$ & $17.3 \pm 12.2$ & $19.5 \pm 11.9$ & 0.032 & $16.0 \pm 11.6$ & $20.9 \pm 12.7$ & $<0.001$ \\
\hline$m \mathrm{MRC}$ Dyspea & $1.7 \pm 1.0$ & $1.6 \pm 1.0$ & $2.1 \pm 1.1$ & $<0.001$ & $1.5 \pm 1.0$ & $2.0 \pm 1.1$ & $<0.001$ \\
\hline$\geq 2$ & $1034(53)$ & $906(50)$ & $128(67)$ & $<0.001^{\#}$ & $621(46)$ & $413(66)$ & $<0.001^{\#}$ \\
\hline \multicolumn{8}{|l|}{ SGRQ-C } \\
\hline Total score & $48.1 \pm 18.2$ & $47.4 \pm 18.1$ & $53.9 \pm 18.2$ & $<0.001$ & $44.9 \pm 18.0$ & $55.1 \pm 16.5$ & $<0.001$ \\
\hline$\geq 50$ points & $927(48)$ & $810(45)$ & $117(62)$ & $<0.001^{\#}$ & $549(41)$ & $378(61)$ & $<0.001^{\#}$ \\
\hline CES-D Score & $11.5 \pm 9.3$ & $11.4 \pm 9.4$ & $12.7 \pm 8.8$ & NS & $11.0 \pm 9.2$ & $12.5 \pm 9.5$ & 0.001 \\
\hline$\geq 16$ & $526(27)$ & $463(26)$ & $63(33)$ & $0.048^{\#}$ & $336(24)$ & $190(31)$ & $0.003^{\#}$ \\
\hline Smokers & $728(36)$ & $658(36)$ & $70(36)$ & $N S^{\#}$ & $516(37)$ & $212(34)$ & $N S^{\#}$ \\
\hline
\end{tabular}

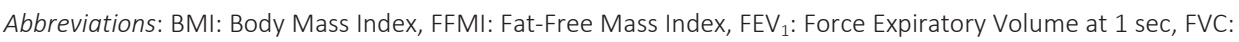
Force Vital Capacity, LAA\%: Low-attenuation area, mMRC: modified Medical Research Council Dyspnea Scale, SGRQ-C: St. George's Respiratory Questionnaire for COPD (40-item version), CES-D: Center for Epidemiologic Screening Test Questionnaire. Data is represented as means \pm SD and percentages (\%). In total 183 patient had undefined extent of emphysema (\% valid percent); Hash tag (\#) denotes statistical significance detected by Pearson Chi-square analysis. Significance level was set at $\mathrm{P}<0.05$. 


\section{Events during follow-up}

A total of 193 subjects (10\%) died during the 3-year follow-up. Non-survivors were older, had more severe airflow limitation, a higher degree of emphysema, higher dyspnea grades, and more impaired health status than survivors (Table 1). In addition, non-survivors had a lower baseline 6MWD, 6MWSpeed, 6MWW, DSP, a higher proportion of stoppers, a higher proportion of patients with EID, and a higher BODE index (Table 2). Similar differences were found between subjects with and without exacerbation-related hospitalization (Tables 1 and 2). Moreover, significant correlations were found amongst survival days, time to hospitalization and the several 6MWTderived variables (Supplementary table S1).

\section{Predictive value of 6MWT-derived variables for mortality and hospitalization}

Tables 3-4 present the threshold values with the best specificity and sensitivity to predict mortality and hospitalization during follow-up for the 6MWD, 6MWSpeed, $6 \mathrm{MWW}, \mathrm{DSP}$, and the BODE index (as a validated reference). The prognostic accuracy of these cut-off points did not vary significantly within different age groups, sex, GOLD stages and the degree of emphysema (Supplementary table S2). Cox's regression analysis unadjusted HR indicated that patients with 6MWD $(\leq 334 \mathrm{~m}), 6 \mathrm{MWSpeed}(\leq 0.9$ $\mathrm{m} / \mathrm{sec}$ ), 6MWW ( $\leq 20000 \mathrm{~m} \mathrm{~kg}), \mathrm{DSP}(\leq 290 \mathrm{~m} \%)$, BODE index ( $\geq 4$ points), EID $\left(\mathrm{SpO}_{2}\right.$ post $\leq 88 \%$ ) or unintended stop(s) have a relative higher risk for mortality (Figure 1). Additionally, the unadjusted HR for the prediction of hospitalization revealed that patients with 6MWD ( $\leq 357 \mathrm{~m}), 6 \mathrm{MWSpeed}(\leq 1.0 \mathrm{~m} / \mathrm{sec}), 6 \mathrm{MWW}(\leq 27000 \mathrm{~m} \cdot \mathrm{kg})$, DSP ( $\leq 310 \mathrm{~m} \%), \mathrm{BODE}$ index ( $\geq 3$ points), EID $\left(\mathrm{SpO}_{2}\right.$ post $\left.\leq 88 \%\right)$, or unintended stop(s) were more likely to be hospitalized during the 3-year follow-up (Figure 2). An adjusted Cox's proportional-hazard regression model for age, sex, BMI, FEV $, F E V_{1} / F V C, S G R Q$, emphysema, and smoking, indicated that the risk for mortality remained high for subjects with impaired 6MWT-derived variables, EID and unintended stop(s) during the 6MWT. Interestingly BODE index did not remain significant after the adjustment (Table 5). Regarding to hospitalization, 6MWW, EID and the BODE index remained significant in the adjusted regression model.

Posterior Cox's regression analyses, which were performed on patients with impaired 6MWT-derived variables according to the proposed cut-off points, incidence of EID and increased BODE index to predict mortality and hospitalization respectively (Tables 3 and 4), demonstrated that unintended stop(s) during the 6MWT retained an additional negative impact on survival (Figure 3). However, this was not observed for hospitalization-free probability (Supplementary figure S1). 


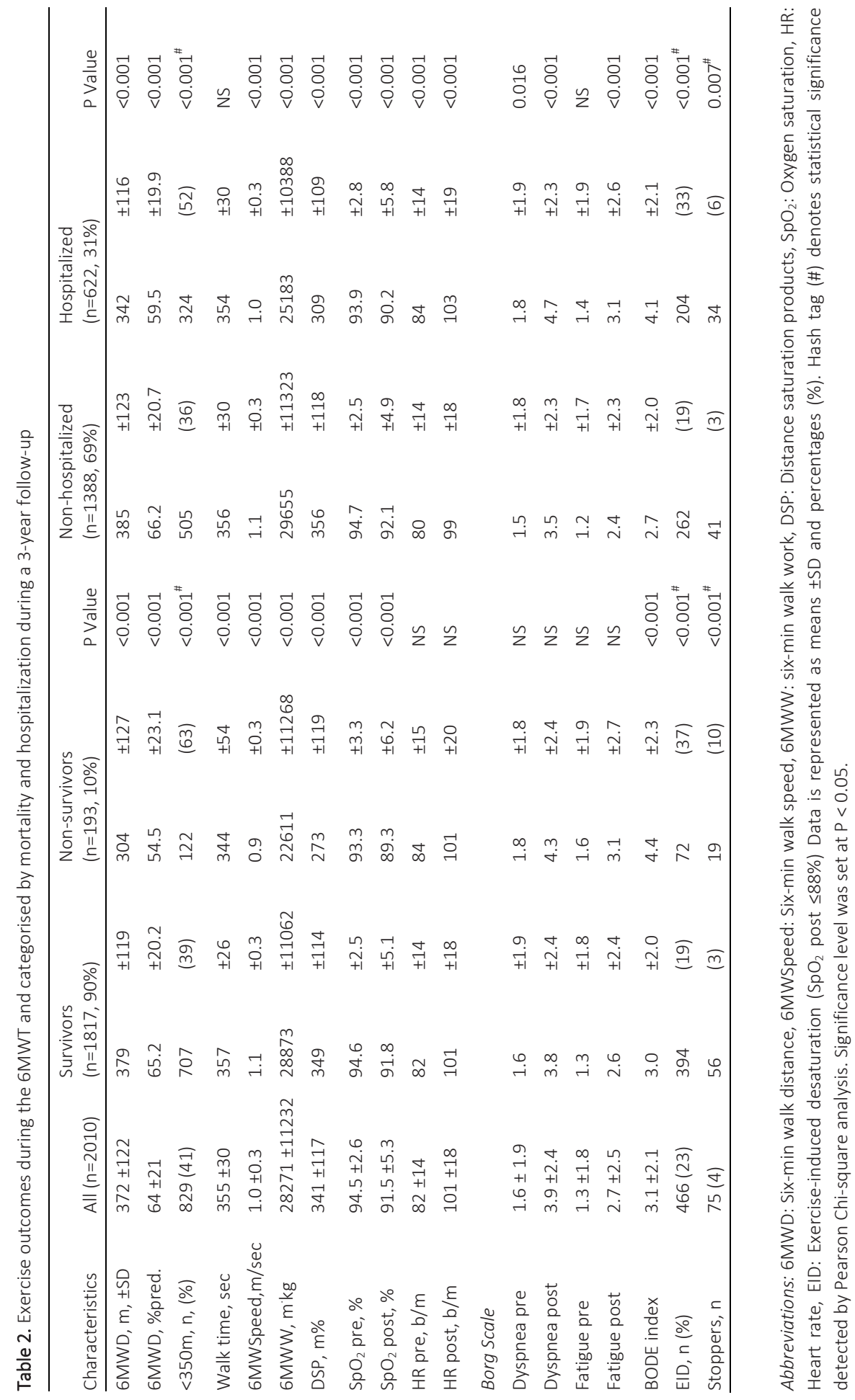


Table 3. Cut-off points and the ROC curve parameters for the prediction of long-term mortality

\begin{tabular}{|c|c|c|c|c|c|c|c|c|}
\hline \multirow[b]{2}{*}{$\begin{array}{l}\text { Predictive } \\
\text { index }\end{array}$} & \multirow[b]{2}{*}{$\begin{array}{l}\text { Best } \\
\text { cut-off }\end{array}$} & \multicolumn{7}{|c|}{ ROC curve parameters } \\
\hline & & $\begin{array}{l}\text { AUC } \\
\text { segment }\end{array}$ & $\begin{array}{l}\text { Sensitivity } \\
\text { (\%) }\end{array}$ & $\begin{array}{l}95 \% \mathrm{Cl} \\
\text { Binomial } \\
\text { exact }\end{array}$ & $\begin{array}{l}\text { Specificity } \\
(\%)\end{array}$ & $\begin{array}{l}95 \% \mathrm{Cl} \\
\text { Binomial } \\
\text { exact }\end{array}$ & $\begin{array}{l}\text { Likeli } \\
\text { LR+ }\end{array}$ & $\begin{array}{l}\text { d Ratio } \\
\text { LR- }\end{array}$ \\
\hline Age, years & $\geq 65$ & 0.63 & 0.62 & $0.54-0.69$ & 0.60 & $0.57-0.62$ & 1.52 & 0.65 \\
\hline $\mathrm{BMI}, \mathrm{kg} / \mathrm{m}^{2}$ & $\leq 23$ & 0.53 & 0.35 & $0.28-0.42$ & 0.75 & $0.73-0.77$ & 1.37 & 0.88 \\
\hline $\mathrm{FEV}_{1}, \%$ pred. & $\leq 43$ & 0.63 & 0.61 & $0.53-0.68$ & 0.62 & $0.60-0.65$ & 1.61 & 0.63 \\
\hline $\mathrm{FEV}_{1} / \mathrm{FVC}, \%$ & $\leq 39$ & 0.58 & 0.50 & $0.43-0.57$ & 0.65 & $0.63-0.67$ & 1.42 & 0.77 \\
\hline SGRQ, score & $\geq 55$ & 0.60 & 0.53 & $0.45-0.60$ & 0.65 & $0.62-0.67$ & 1.49 & 0.73 \\
\hline 6MWD, m & $\leq 334$ & 0.67 & 0.62 & $0.54-0.69$ & 0.65 & $0.63-0.67$ & 1.76 & 0.59 \\
\hline 6MWSpeed, m/sec & $\leq 0.9$ & 0.67 & 0.57 & $0.49-0.64$ & 0.69 & $0.66-0.71$ & 1.80 & 0.63 \\
\hline $6 \mathrm{MWW}, \mathrm{m} k \mathrm{~kg}$ & $\leq 20000$ & 0.66 & 0.46 & $0.39-0.53$ & 0.79 & $0.77-0.81$ & 2.17 & 0.68 \\
\hline DSP, $\mathrm{m} \%$ & $\leq 290$ & 0.68 & 0.58 & $0.51-0.65$ & 0.72 & $0.70-0.74$ & 2.06 & 0.59 \\
\hline BODE index & $\geq 4$ & 0.67 & 0.60 & $0.53-0.67$ & 0.63 & $0.61-0.66$ & 1.64 & 0.63 \\
\hline
\end{tabular}

Abbreviations: Please see legends in Tables 1 and 2.

Table 4. Cut-off points and the ROC curve parameters for the prediction of long-term hospitalization

\begin{tabular}{|c|c|c|c|c|c|c|c|c|}
\hline \multirow[b]{3}{*}{$\begin{array}{l}\text { Predictive } \\
\text { index }\end{array}$} & \multirow[b]{3}{*}{$\begin{array}{l}\text { Best } \\
\text { cut-off }\end{array}$} & \multicolumn{7}{|c|}{ ROC curve parameters } \\
\hline & & \multirow[b]{2}{*}{$\begin{array}{l}\text { AUC } \\
\text { segment }\end{array}$} & \multirow[b]{2}{*}{$\begin{array}{l}\text { Sensitivity } \\
(\%)\end{array}$} & \multirow{2}{*}{$\begin{array}{l}95 \% \mathrm{Cl} \\
\text { Binomial } \\
\text { exact }\end{array}$} & \multirow[b]{2}{*}{$\begin{array}{l}\text { Specificity } \\
(\%)\end{array}$} & \multirow{2}{*}{$\begin{array}{l}95 \% \mathrm{Cl} \\
\text { Binomial } \\
\text { exact }\end{array}$} & \multicolumn{2}{|c|}{ Likelihood Ratio } \\
\hline & & & & & & & $\mathrm{LR}+$ & LR- \\
\hline Age, years & $\geq 64$ & 0.54 & 0.53 & $0.49-0.57$ & 0.55 & $0.53-0.58$ & 1.20 & 0.84 \\
\hline $\mathrm{BMI}, \mathrm{kg} / \mathrm{m}^{2}$ & $\leq 23$ & 0.54 & 0.32 & $0.28-0.36$ & 0.75 & $0.73-0.78$ & 1.29 & 0.90 \\
\hline $\mathrm{FEV}_{1}, \%$ pred. & $\leq 44$ & 0.67 & 0.60 & $0.56-0.64$ & 0.65 & $0.63-0.68$ & 1.73 & 0.61 \\
\hline $\mathrm{FEV}_{1} / \mathrm{FVC}, \%$ & $\leq 45$ & 0.63 & 0.67 & $0.63-0.70$ & 0.52 & $0.49-0.54$ & 1.38 & 0.64 \\
\hline SGRQ, score & $\geq 45$ & 0.66 & 0.73 & $0.69-0.76$ & 0.50 & $0.47-0.53$ & 1.45 & 0.55 \\
\hline 6MWD, m & $\leq 357$ & 0.60 & 0.54 & $0.50-0.58$ & 0.62 & $0.59-0.65$ & 1.40 & 0.75 \\
\hline 6MWSpeed, m/sec & $\leq 1.0$ & 0.60 & 0.56 & $0.52-0.60$ & 0.58 & $0.56-0.61$ & 1.35 & 0.75 \\
\hline $6 \mathrm{MWW}, \mathrm{m} k \mathrm{~kg}$ & $\leq 27000$ & 0.62 & 0.61 & $0.58-0.65$ & 0.56 & $0.53-0.59$ & 1.40 & 0.69 \\
\hline $\mathrm{DSP}, \mathrm{m} \%$ & $\leq 310$ & 0.62 & 0.52 & $0.48-0.56$ & 0.67 & $0.64-0.69$ & 1.55 & 0.73 \\
\hline BODE index & $\geq 3$ & 0.69 & 0.57 & $0.53-0.61$ & 0.69 & $0.67-0.72$ & 1.85 & 0.62 \\
\hline
\end{tabular}

Abbreviations: Please see legends in Tables 1 and 2. 

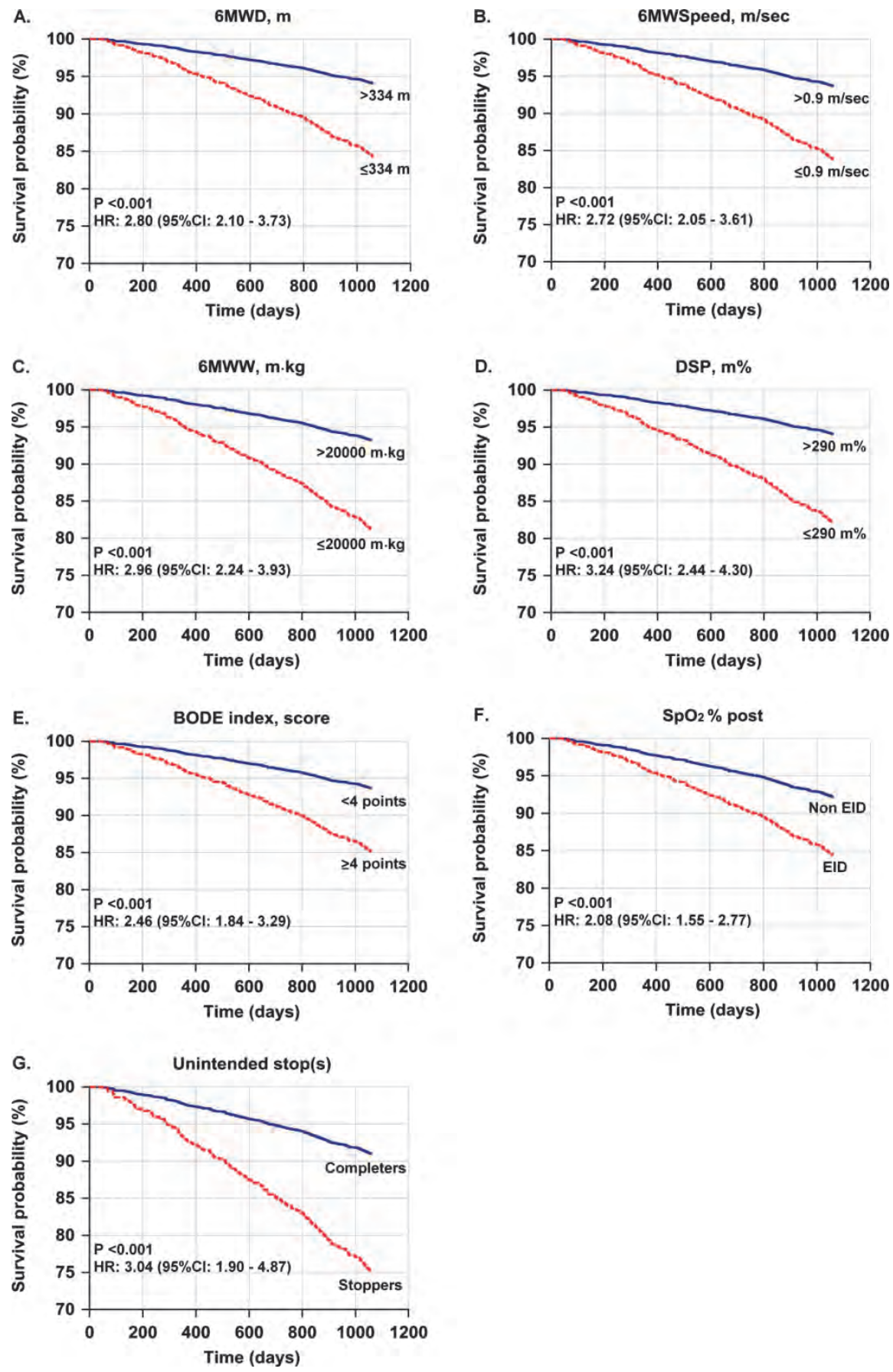

Figure 1. Kaplan-Meier survival curves referring to impaired 6MWT-derived variables

The cut-off values used are indicated within the plots. Unadjusted hazard ratio (HR) and 95\% confidence intervals $(95 \% \mathrm{Cl})$ were calculated by Cox's proportional-hazard regression. Significance set on $\mathrm{P}<0.05$. 
A

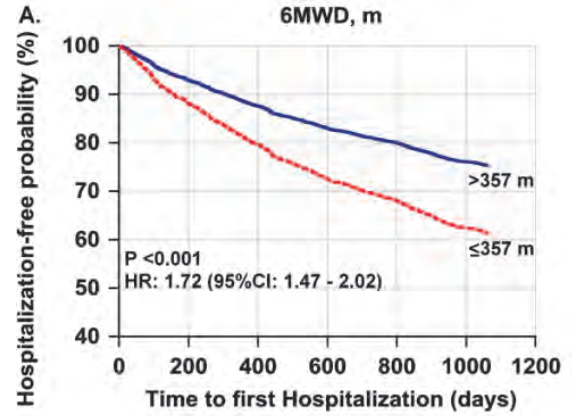

c.

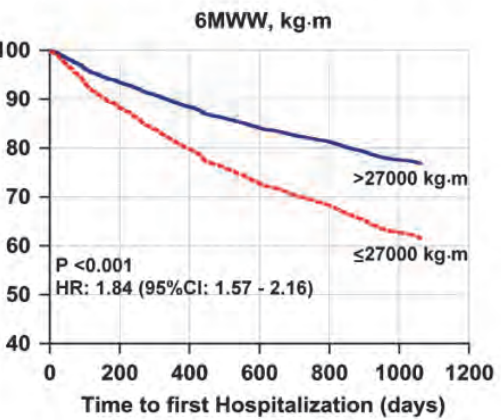

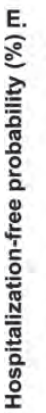

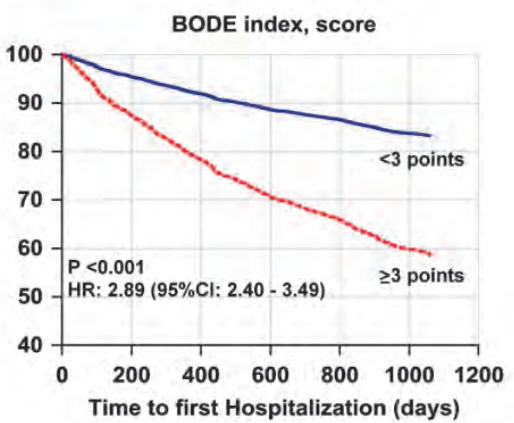

G.

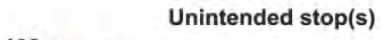

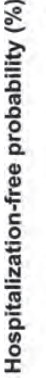

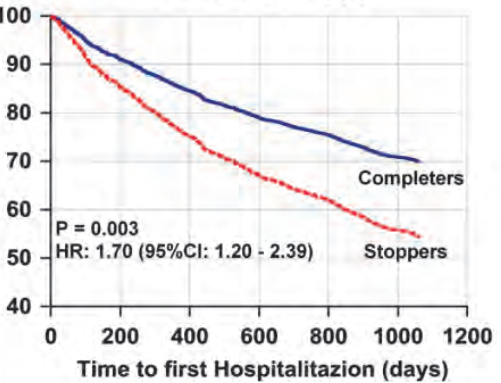

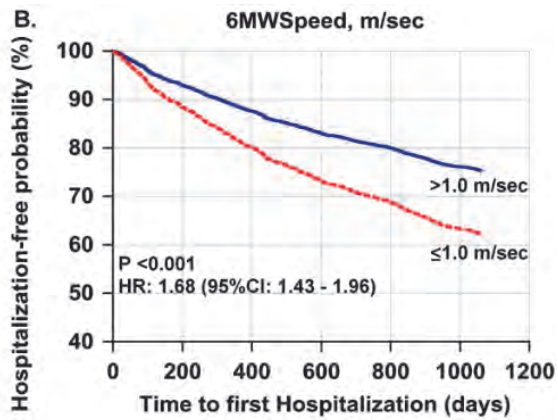
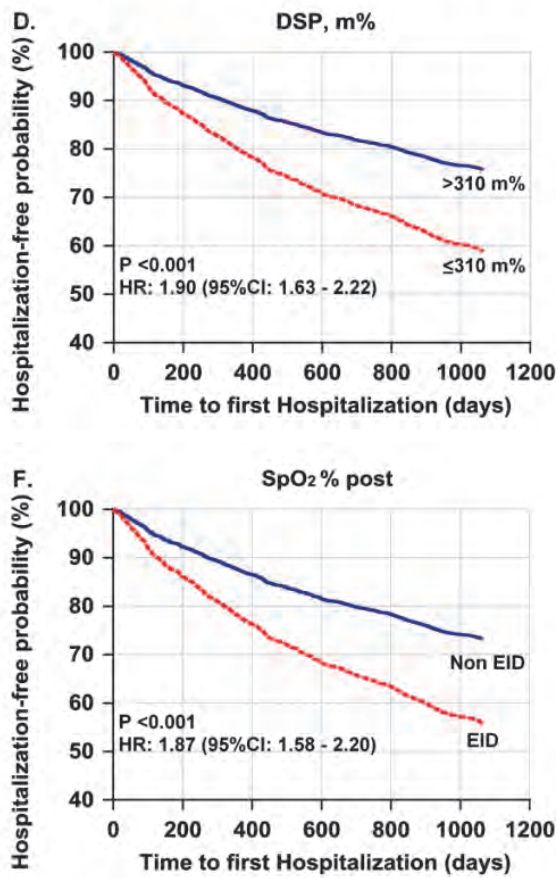

Figure 2. Kaplan-Meier curves for hospitalization referring to impaired 6MWT-derived variables

The cut-off values used are indicated within the plots. Unadjusted hazard ratio (HR) and $95 \%$ confidence intervals $(95 \% \mathrm{Cl})$ were calculated by Cox's proportional-hazard regression. Significance set on $\mathrm{P}<0.05$. 


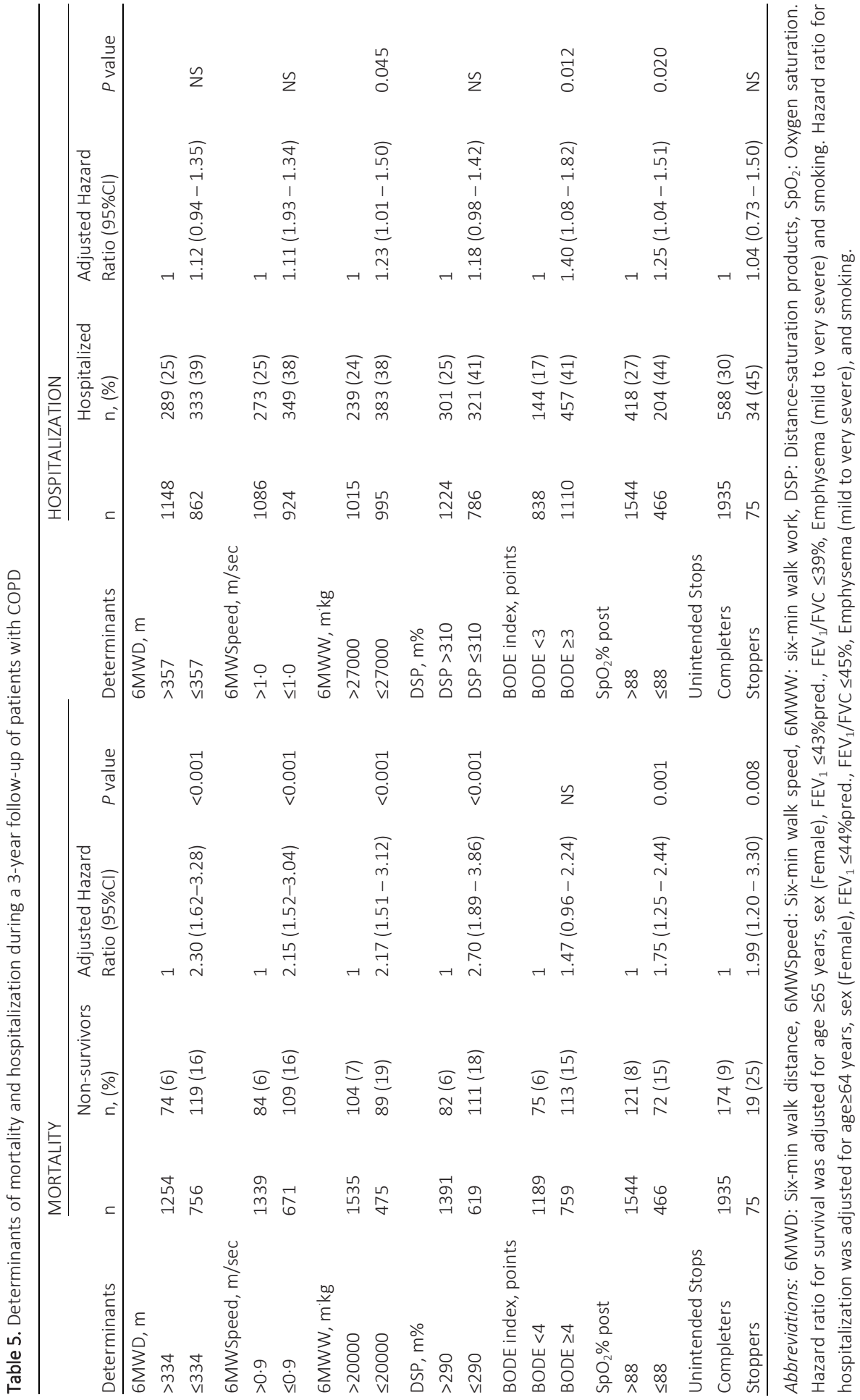




\section{DISCUSSION}

This study explores the prognostic value of the 6MWT-derived variables including 6MWD, 6MWSpeed, 6MWW, DSP, EID, and unintended stop(s) for the prediction of mortality and hospitalization during a 3-year follow-up in patients with COPD. The 6MWD data of the ECLIPSE has been already investigated ${ }^{2,22,23}$, however the essential novelty of our approach is that we demonstrated the 6MWT-derived variables in association with mortality and hospitalization.

We found that unintended stop(s) during the 6MWT can be a prognostic factor for mortality and hospitalization (Figures 1 and 2). Interestingly, unintended stop(s) together with the rest of 6MWT-derived variables can refine the prognosis to predict mortality (Figure 3). Unintended stop(s), which can also lead to withdrawal from the 6MWT, may reflect the overall impact of poor 6MWT-derived variables and increased BODE index on patients' functional capacity. However, unintended stop(s) combined with impaired 6MWT-derived variables did not lend support in the prediction of hospitalization (Supplementary figure S1). A possible explanation could be that patients with poor 6MWT-derived variables of 6MWD, 6MWSpeed, 6MWW, DSP, incidence of EID and increased BODE index, usually experience the first severe exacerbation related to hospitalization before there is an impact on their ability to keep a continuous pace during the 6MWT. Using an adjusted Cox's proportional-hazard regression model, the prognostic value of unintended stops to predict mortality remained after adjustment for age, sex, BMI, $\mathrm{FEV}_{1}, \mathrm{FEV}_{1} / \mathrm{FVC}$, SGRQ, emphysema, and smoking suggesting that unintended stop(s) can be an important prognostic outcome for survival (Table 5). To the best of our knowledge, this is the first study that demonstrates the prognostic value of unintended stop(s) during the 6MWT in patients with COPD and suggests that more attention should be given to this outcome for the identification of high-risk patients.

\section{Comparison with other studies}

The 6MWSpeed has been already shown as a functional capacity indicator in 511 patients with mild to severe COPD. ${ }^{10}$ A mean $6 \mathrm{MWSpeed}<1.0 \mathrm{~m} / \mathrm{s}$ has been related to disability, hospitalization and decreased survival in older adults. ${ }^{5,10} \mathrm{~A}$ mean 6MWSpeed $\leq 0.9 \mathrm{~m} / \mathrm{sec}$ increased the risk of death while $6 \mathrm{MWSpeed} \leq 1.0 \mathrm{~m} / \mathrm{sec}$ increased the risk of hospitalization in our patients with COPD, respectively. The present study is the first one which provides certain cut-off points for the 6MWSpeed with prognostic value on mortality (Figure 1B) and hospitalization (Figure 2B) in COPD patients.

In the study of Chuang and colleagues ${ }^{25}, 6 \mathrm{MWW}$ was used to assess work and energy expenditure during the 6MWT demonstrating a better correlation with the anaerobic threshold, peak oxygen uptake, DLCO and vital capacity when compared to 
6MWD. Carter and colleagues ${ }^{12}$ reported that $6 \mathrm{MWW}$ is an improved outcome measure with a solid physiologic foundation for the assessment of functional capacity in COPD. DSP variable has also been shown to be a potential indicator of functional status in patients with sarcoidosis ${ }^{26}$ while DSP $<200 \mathrm{~m} \%$ has been associated with a seven-fold greater risk of 12-month mortality in patients with idiopathic pulmonary fibrosis (IPF). ${ }^{14}$ The prognostic value of 6MWW and DSP to predict mortality and hospitalization has also been confirmed by our findings (Figures $1-2 C \& 2 D$ ).

Other studies have demonstrated that the levels of oxygen saturation and incidence of EID during the 6MWT could improve the predictive ability of the 6MWD in COPD patients. ${ }^{27,} 28$ In this study, the occurrence of EID was also an indicator for increased mortality and hospitalization. Beside the prognostic value of worse 6MWT-derived variables, we also observed that older patients with lower $\mathrm{FEV}_{1}$ and increased scores of SGRQ have higher risk for mortality and hospitalization (Supplementary figure S2). Regarding to the BODE index, a study of Ong and colleagues ${ }^{18}$ in 127 COPD patients who were followed up for 16 months demonstrated the BODE index as a better predictor of hospitalizations for COPD than the $\mathrm{FEV}_{1}$ alone. Previously, BODE score of 3 points or higher has been associated to mortality and exacerbation rates in patients with COPD. ${ }^{29}$ Our findings suggested that increased BODE score was an independent predictor of mortality and hospitalization in our large cohort of patients with COPD (Figures $1-2 \mathrm{E} \&$ $2 \mathrm{~F})$.

A Cox's regression analysis with proportional hazard model for the prediction of 3year mortality and hospitalization adjusted for age, sex, BMI, FEV $1, F E V_{1} / F V C, S G R Q$, emphysema, and smoking indicated that patients with impaired 6MWT-derived variables have increased risk of death (Table 5). After this adjustment, the cut-off point used for DSP ( $\leq 290 \mathrm{~m} \%$ ) was the best predictor of mortality (Hazard Ratio: 2.70) while, surprisingly, the BODE index did not remain significant. We assume that the inclusion of $S G R Q$ and $B M I$ in the adjustment of regression model reduced the BODE index significance as a result of variables' interrelation. In terms of hospitalization, patients with worse 6MWW, BODE index and EID had higher risk of exacerbation-related hospitalization (Table 5). The cut-off point of the BODE index ( $\geq 3$ points) appeared to be the strongest predictor (Hazard Ratio: 1.40). Our adjusted proportional hazard models demonstrated that DSP is the stronger predictor of mortality while the BODE index is the most sensitive tool for the prediction of hospitalization compared to the 6MWTderived variables. 
A.

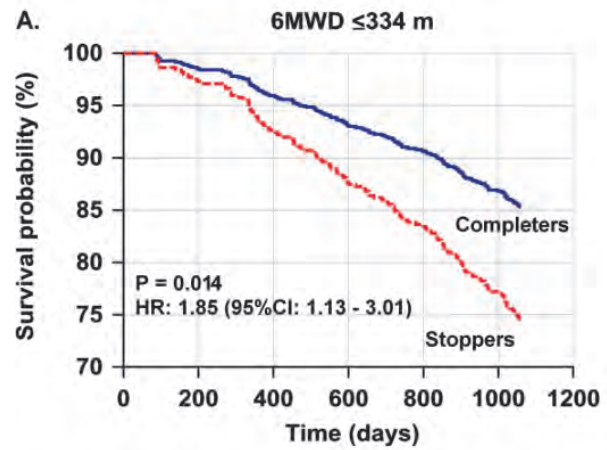

c.

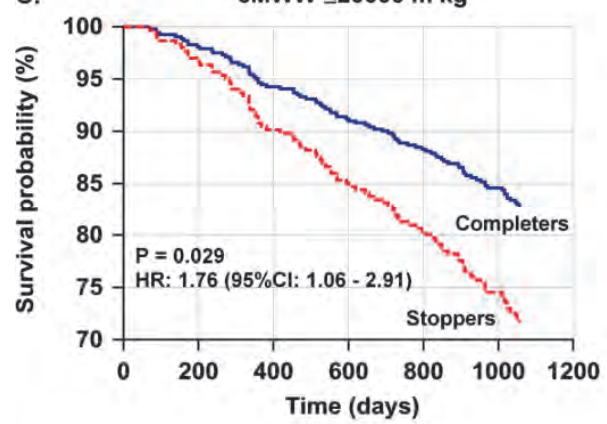

E.

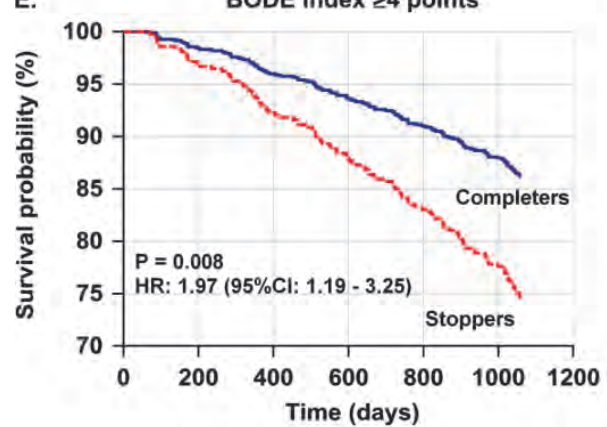

B.

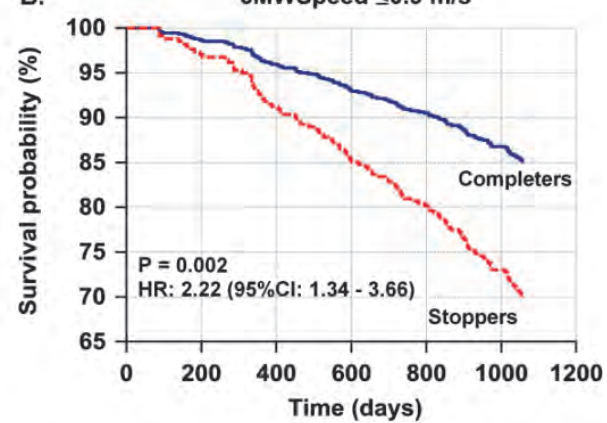

D.

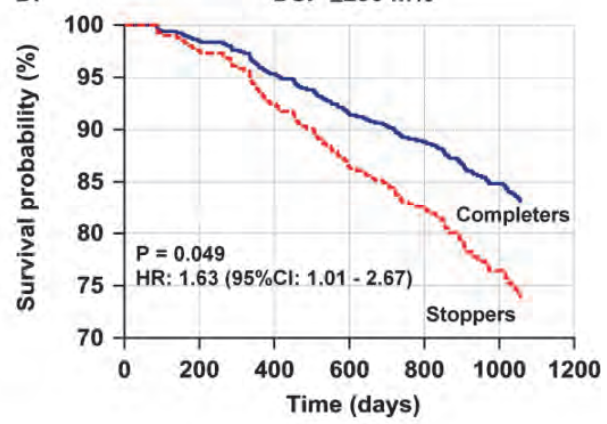

F.

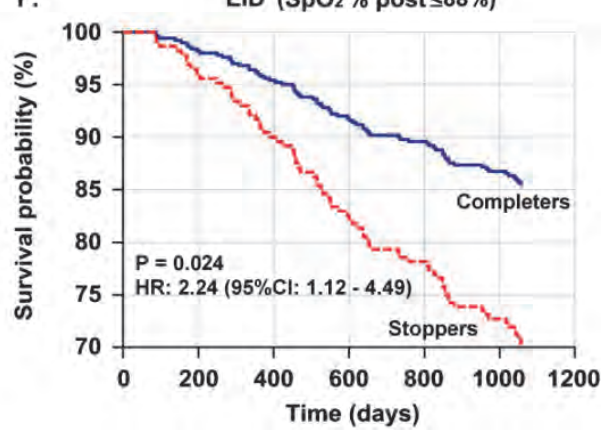

Figure 3. Kaplan-Meier survival curves referring to unintended stop(s) during 6 MWT of patients with impaired 6 MWT-derived variables

Unintended stops (Completers/Stoppers) during the 6 MWT were used for the estimation of survival probability (\%). Unadjusted Hazard ratio (HR) and $95 \%$ confidence intervals $(95 \% \mathrm{Cl})$ were calculated by Cox's proportional-hazard regression. Significance level was set at $\mathrm{P}<0.05$.

\section{Strengths and weaknesses}

There are certain limitations to our study. First, the 6MWT screening was performed only once at baseline, while the 2014 ERS/ATS Technical Standard for field walking tests suggest the conduct of two $6 \mathrm{MWT} .{ }^{30}$ At the time of designating the ECLIPSE study, the 
2002 ATS statement on 6MWT was used, which did not recommend a practice walk. The 6MWT-derived variables, such as oxygen saturation, are reproducible. ${ }^{31}$ Obviously, 6MWW and DSP may have been a little bit higher as a second 6MWT would increase the 6MWD in about $80 \%$ of the patients with COPD. ${ }^{31}$ Second, the number of the unintended stops during the 6MWT is not available; nevertheless, the total walking time indicated whether or not patients stopped to rest during the 6MWT. Third, the EID was defined based on the $\mathrm{SpO}_{2}$ at the end of the test $\left(\mathrm{SpO}_{2}\right.$ post) and not the $\mathrm{SpO}_{2}$ nadir which can lead to an overestimation of the DSP in patients who exhibit EID during the 6MWT. Even though $\mathrm{SpO}_{2}$ nadir and the $\mathrm{SpO}_{2}$ post for most patients with chronic respiratory disease are relatively similar during the 6MWT with differences ranging from $1 \%$ to $10 \%^{32}$, the use of $\mathrm{SpO}_{2}$ nadir would be optimal absent the biases. Concerning the diagnostic accuracy, the area under the ROC curve quantifies the overall ability of the tested variables to discriminate between patients who have a good or poor prognosis. Even though the diagnostic accuracy of the 6MWT-derived variables can be considered as sufficient based on the AUC $(\geq 0.60)^{33}$, a range of values is rather low $(<0.60)$. In turn, omissions errors and commission errors may occur. ${ }^{34}$

Despite these limitations, this study contributes with important information about the 6MWT-derived variables and the BODE index in respect of their relationship to mortality and hospitalization in patients with COPD. Our findings may help clinicians to better predict the survival and hospitalization in patients with COPD. Additionally, unintended stop(s) during the 6MWT may represent an advanced stage of disease severity in which enrolment in a pulmonary rehabilitation program could be urgently recommended.

\section{CONCLUSIONS}

The 6MWT-derived variables of 6MWD, 6MWSpeed, 6MWW, DSP, incidence of EID, unintended stop(s) and the BODE index are important predictors of long-term mortality and hospitalization in patients with COPD. New insights into the 6MWT-derived variables indicate the DSP as the strongest predictor of mortality and the BODE index as the most sensitive tool for assessing the risk of hospitalization. Besides the poor 6MWTderived variables and the BODE index, novel evidences suggesting that unintended stop(s) during the 6MWT can refine the prognosis of mortality in COPD. Obviously, these findings need to be reproduced by others and reliability needs to be studied. 


\section{REFERENCES}

1. Solway S, Brooks D, Lacasse $Y$ and Thomas S. A qualitative systematic overview of the measurement properties of functional walk tests used in the cardiorespiratory domain. Chest. 2001; 119: 256-70.

2. Spruit MA, Watkins ML, Edwards LD, et al. Determinants of poor 6-min walking distance in patients with COPD: The ECLIPSE cohort. Respir Med. 2010; 104: 849-57.

3. Singh SJ, Puhan MA, Andrianopoulos V, et al. An official systematic review of the European Respiratory Society/American Thoracic Society: measurement properties of field walking tests in chronic respiratory disease. Eur Respir J. 2014; 44: 1447-78.

4. Cote CG, Casanova C, Marin JM, et al. Validation and comparison of reference equations for the 6-min walk distance test. Eur Respir J. 2008; 31: 571-8.

5. Studenski S, Perera S, Patel K, et al. Gait speed and survival in older adults. JAMA. 2011; 305: 50-8.

6. Gerardi DA, Lovett L, Benoit-Connors ML, Reardon JZ and ZuWallack RL. Variables related to increased mortality following out-patient pulmonary rehabilitation. Eur Respir J. 1996; 9: 431-5.

7. Wong R, Sibley KM, Hudani M, et al. Characteristics of people with chronic lung disease who rest during the six-minute walk test. Arch Phys Med Rehabil. 2010; 91: 1765-9.

8. Kessler R, Faller M, Fourgaut G, Mennecier B and Weitzenblum E. Predictive factors of hospitalization for acute exacerbation in a series of 64 patients with chronic obstructive pulmonary disease. Am J Respir Crit Care Med. 1999; 159: 158-64.

9. Cesari M, Kritchevsky SB, Penninx BW, et al. Prognostic value of usual gait speed in well-functioning older people--results from the Health, Aging and Body Composition Study. J Am Geriatr Soc. 2005; 53: 167580.

10. Ilgin D, Ozalevli S, Kilinc O, Sevinc C, Cimrin AH and Ucan ES. Gait speed as a functional capacity indicator in patients with chronic obstructive pulmonary disease. Ann Thorac Med. 2011; 6: 141-6.

11. Vestergaard S, Patel KV, Bandinelli S, Ferrucci L and Guralnik JM. Characteristics of 400-meter walk test performance and subsequent mortality in older adults. Rejuvenation Res. 2009; 12: 177-84.

12. Carter R, Holiday DB, Nwasuruba C, Stocks J, Grothues C and Tiep B. 6-minute walk work for assessment of functional capacity in patients with COPD. Chest. 2003; 123: 1408-15.

13. Poersch K, Berton DC, Canterle DB, et al. Six-minute walk distance and work relationship with incremental treadmill cardiopulmonary exercise test in COPD. Clin Respir J. 2013; 7: 145-52.

14. Lettieri CJ, Nathan SD, Browning RF, Barnett SD, Ahmad S and Shorr AF. The distance-saturation product predicts mortality in idiopathic pulmonary fibrosis. Respir Med. 2006; 100: 1734-41.

15. Golpe R, Perez-de-Llano LA, Mendez-Marote L and Veres-Racamonde A. Prognostic value of walk distance, work, oxygen saturation, and dyspnea during 6-minute walk test in COPD patients. Respir Care. 2013; 58: 1329-34.

16. Vestbo J, Anderson W, Coxson HO, et al. Evaluation of COPD Longitudinally to Identify Predictive Surrogate End-points (ECLIPSE). Eur Respir J. 2008; 31: 869-73.

17. Celli BR, Cote CG, Marin JM, et al. The body-mass index, airflow obstruction, dyspnea, and exercise capacity index in chronic obstructive pulmonary disease. N Engl J Med. 2004; 350: 1005-12.

18. Ong KC, Earnest A and Lu SJ. A multidimensional grading system (BODE index) as predictor of hospitalization for COPD. Chest. 2005; 128: 3810-6.

19. Rabe KF, Hurd S, Anzueto A, et al. Global strategy for the diagnosis, management, and prevention of chronic obstructive pulmonary disease: GOLD executive summary. Am J Respir Crit Care Med. 2007; 176: 532-55.

20. ATS. ATS statement: guidelines for the six-minute walk test. Am J Respir Crit Care Med. 2002; 166: 111-7.

21. Holland AE, Spruit MA, Troosters T, et al. An official European Respiratory Society/American Thoracic Society Technical Standard: field walking tests in chronic respiratory disease. Eur Respir J. 2014; 44(6): 1428-46.

22. Spruit MA, Polkey MI, Celli B, et al. Predicting outcomes from 6-minute walk distance in chronic obstructive pulmonary disease. J Am Med Dir Assoc. 2012; 13: 291-7. 


\section{Chapter 4}

23. Polkey MI, Spruit MA, Edwards LD, et al. Six-minute-walk test in chronic obstructive pulmonary disease: minimal clinically important difference for death or hospitalization. Am J Respir Crit Care Med. 2013; 187: 382-6.

24. DeLong ER, DeLong DM and Clarke-Pearson DL. Comparing the areas under two or more correlated receiver operating characteristic curves: a nonparametric approach. Biometrics. 1988; 44: 837-45.

25. Chuang ML, Lin IF and Wasserman K. The body weight-walking distance product as related to lung function, anaerobic threshold and peak VO2 in COPD patients. Respir Med. 2001; 95: 618-26.

26. Alhamad EH, Shaik SA, Idrees MM, Alanezi MO and Isnani AC. Outcome measures of the 6 minute walk test: relationships with physiologic and computed tomography findings in patients with sarcoidosis. BMC Pulm Med. 2010; 10: 42.

27. Casanova C, Cote C, Marin JM, et al. Distance and oxygen desaturation during the 6-min walk test as predictors of long-term mortality in patients with COPD. Chest. 2008; 134: 746-52.

28. Takigawa N, Tada A, Soda R, et al. Distance and oxygen desaturation in 6-min walk test predict prognosis in COPD patients. Respir Med. 2007; 101: 561-7.

29. Marin JM, Carrizo SJ, Casanova C, et al. Prediction of risk of COPD exacerbations by the BODE index. Respir Med. 2009; 103: 373-8.

30. Holland AE, Spruit MA, Troosters T, et al. An official European Respiratory Society/American Thoracic Society technical standard: field walking tests in chronic respiratory disease. Eur Respir J. 2014; 44: 142846.

31. Hernandes NA, Wouters EF, Meijer K, Annegarn J, Pitta F and Spruit MA. Reproducibility of 6-minute walking test in patients with COPD. Eur Respir J. 2011; 38: 261-7.

32. Fiore C, Lee A, McDonald C, Hill C and Holland A. Should oxyhaemoglobin saturation be monitored continuously during the 6-minute walk test? Chron Respir Dis. 2011; 8: 181-4.

33. Šimundić A. Measures of diagnostic accuracy: basic definitions. Ejifcc 2009; 19:203-11

34. Lobo MJ, Jimenez-Valverde A and Real R. AUC: a misleading measure of the performance of predictive distribution models. Global Ecol Biogeogr. 2007: 1-7. 


\section{SUPPLEMENTAL MATERIAL}

Table S1. Correlation matrix amongst survival days, time to hospitalization and several 6MWT derived outcomes

\begin{tabular}{|c|c|c|c|c|c|c|c|c|c|}
\hline & $\begin{array}{l}\text { Survival } \\
\text { days }\end{array}$ & $\begin{array}{l}\text { Time to } \\
\text { hospital- } \\
\text { ization }\end{array}$ & $\begin{array}{l}\text { 6MWD, } \\
\mathrm{m}\end{array}$ & $\begin{array}{l}\text { 6MW- } \\
\text { Speed, } \\
\text { m/sec }\end{array}$ & $\begin{array}{l}6 \mathrm{MWW} \\
\mathrm{m} k \mathrm{~kg}\end{array}$ & $\begin{array}{l}\text { DSP, } \\
m \%\end{array}$ & $\begin{array}{l}\text { BODE, } \\
\text { score }\end{array}$ & $\begin{array}{l}\mathrm{SpO}_{2} \% \\
\text { post }\end{array}$ & $\begin{array}{l}\text { Unintended } \\
\text { Stops }\end{array}$ \\
\hline Survival days & 1 & $.134 * *$ & $.149 * *$ & $.141^{* *}$ & $.141^{* *}$ & $.158 * *$ & $-.150 * *$ & $.132 * *$ & $-.103 * *$ \\
\hline $\begin{array}{l}\text { Time to } \\
\text { hospitalization }\end{array}$ & $.134^{* *}$ & 1 & $.142 * *$ & $.148 * *$ & $.161 * *$ & $.154 * *$ & $-.192 * *$ & $.111 * *$ & $-.062 *$ \\
\hline $\begin{array}{l}\text { 6MWD, } \\
\mathrm{m}\end{array}$ & $.149 * *$ & $.142 * *$ & 1 & $.981 * *$ & $.809 * *$ & $.987^{* *}$ & $-.679 * *$ & $.191 * *$ & $-.285 * *$ \\
\hline $\begin{array}{l}\text { 6MWSpeed, } \\
\mathrm{m} / \mathrm{sec}\end{array}$ & $.141 * *$ & $.148 * *$ & $.981 * *$ & 1 & $.793 * *$ & $.969 * *$ & $-.661 * *$ & $.183 * *$ & $-.202 * *$ \\
\hline $\begin{array}{l}\text { 6MWW, } \\
\mathrm{m} k \mathrm{~kg}\end{array}$ & $.141 * *$ & $.161 * *$ & $.809 * *$ & $.793 * *$ & 1 & $.800 * *$ & $-.621 * *$ & $.156 * *$ & $.267^{* *}$ \\
\hline $\begin{array}{l}\text { DSP, } \\
m \%\end{array}$ & $.158 * *$ & $.154 * *$ & $.987 * *$ & $.969 * *$ & $.800 * *$ & 1 & $-.697 * *$ & $.340 * *$ & $-.286 * *$ \\
\hline $\begin{array}{l}\text { BODE, } \\
\text { score }\end{array}$ & $-.150 * *$ & $-.192 * *$ & $-.679 * *$ & $-.661^{* *}$ & $-.621^{* *}$ & $-.697 * *$ & 1 & $-.321^{* *}$ & $-.258 * *$ \\
\hline $\begin{array}{l}\mathrm{SpO}_{2} \% \\
\text { post }\end{array}$ & $.132 * *$ & $.111^{* *}$ & $.191 * *$ & $.183 * *$ & $.156^{* *}$ & $.340 * *$ & $-.321 * *$ & 1 & $-.072 * *$ \\
\hline $\begin{array}{l}\text { Unintended } \\
\text { Stops }\end{array}$ & $-.103 * *$ & $-.062 *$ & $-.285 * *$ & $-.202 * *$ & $-.267 * *$ & $-.286 * *$ & $.258 * *$ & $-.072 * *$ & 1 \\
\hline
\end{tabular}

Abbreviations: 6MWD: Six-min walk distance, 6MWSpeed: Six-min walk speed, 6MWW: six-min walk work, DSP: Distance saturation products, $\mathrm{SpO}_{2}$ : Oxygen saturation. 


\section{Chapter 4}

Table S2. ROC curves for the prediction of mortality and hospitalization

\begin{tabular}{|c|c|c|c|c|c|c|c|c|c|c|c|}
\hline \multirow{3}{*}{$\begin{array}{l}\text { PREDICTORS OF } \\
\text { MORTALITY }\end{array}$} & \multicolumn{11}{|c|}{ AREA UNDER THE CURVE (AUC) } \\
\hline & \multicolumn{3}{|c|}{ Age, years } & \multicolumn{2}{|l|}{ Sex } & \multicolumn{3}{|c|}{ GOLD Stages } & \multicolumn{3}{|c|}{ Emphysema extend } \\
\hline & $<55$ & $55-65$ & $>65$ & M & $\mathrm{F}$ & II & III & IV & $<5 \%$ & $5-50 \%$ & $>50 \%$ \\
\hline $\begin{array}{l}\text { 6MWD } \\
\leq 334 \mathrm{~m}\end{array}$ & 0.60 & 0.63 & 0.62 & 0.62 & 0.67 & 0.60 & 0.62 & 0.59 & 0.65 & 0.62 & 0.65 \\
\hline $\begin{array}{l}\text { 6MWSpeed } \\
\leq 0.9 \mathrm{~m} / \mathrm{sec}\end{array}$ & 0.61 & 0.61 & 0.63 & 0.61 & 0.67 & 0.60 & 0.62 & 0.59 & 0.62 & 0.62 & 0.64 \\
\hline $\begin{array}{l}\text { 6MWW } \\
\leq 20000 \mathrm{~m} k \mathrm{~kg}\end{array}$ & 0.60 & 0.64 & 0.60 & 0.60 & 0.69 & 0.55 & 0.63 & 0.62 & 0.61 & 0.57 & 0.66 \\
\hline $\begin{array}{l}\text { DSP } \\
\leq 290 \mathrm{~m} \%\end{array}$ & 0.62 & 0.63 & 0.64 & 0.64 & 0.68 & 0.60 & 0.63 & 0.61 & 0.60 & 0.66 & 0.66 \\
\hline $\begin{array}{l}\text { BODE index } \\
\geq 4 \text { score }\end{array}$ & 0.60 & 0.63 & 0.61 & 0.59 & 0.68 & 0.54 & 0.60 & 0.52 & 0.61 & 0.56 & 0.64 \\
\hline $\begin{array}{l}\text { EID } \\
\left(\mathrm{SpO}_{2} \text { post } \leq 88 \%\right)\end{array}$ & 0.53 & 0.55 & 0.59 & 0.58 & 0.58 & 0.53 & 0.56 & 0.57 & 0.53 & 0.60 & 0.56 \\
\hline Unintended stops & s 0.53 & 0.56 & 0.52 & 0.52 & 0.56 & 0.50 & 0.52 & 0.59 & 0.56 & 0.52 & 0.54 \\
\hline \multicolumn{12}{|l|}{ HOSPITALIZATION } \\
\hline $\begin{array}{l}\text { 6MWD } \\
\leq 357 \mathrm{~m}\end{array}$ & 0.63 & 0.57 & 0.57 & 0.57 & 0.59 & 0.55 & 0.56 & 0.51 & 0.55 & 0.55 & 0.59 \\
\hline $\begin{array}{l}\text { 6MWSpeed } \\
\leq 1.0 \mathrm{~m} / \mathrm{sec}\end{array}$ & 0.63 & 0.57 & 0.56 & 0.56 & 0.59 & 0.56 & 0.55 & 0.52 & 0.55 & 0.54 & 0.59 \\
\hline $\begin{array}{l}\text { 6MWW } \\
\leq 27000 \mathrm{~m} k \mathrm{~kg}\end{array}$ & 0.57 & 0.60 & 0.58 & 0.58 & 0.60 & 0.59 & 0.55 & 0.53 & 0.54 & 0.58 & 0.57 \\
\hline $\begin{array}{l}\text { DSP } \\
\leq 310 \mathrm{~m} \%\end{array}$ & 0.63 & 0.59 & 0.58 & 0.58 & 0.61 & 0.58 & 0.55 & 0.51 & 0.55 & 0.57 & 0.59 \\
\hline $\begin{array}{l}\text { BODE index } \\
\geq 3 \text { score }\end{array}$ & 0.67 & 0.64 & 0.63 & 0.64 & 0.63 & 0.59 & 0.56 & 0.50 & 0.60 & 0.63 & 0.59 \\
\hline $\begin{array}{l}\text { EID } \\
\left(\mathrm{SpO}_{2} \text { post } \leq 88 \%\right)\end{array}$ & 0.56 & 0.57 & 0.57 & 0.59 & 0.54 & 0.55 & 0.54 & 0.51 & 0.54 & 0.55 & 0.55 \\
\hline Unintended stops & s 0.50 & 0.53 & 0.51 & 0.51 & 0.51 & 0.51 & 0.50 & 0.50 & 0.51 & 0.51 & 0.52 \\
\hline
\end{tabular}

Abbreviations: 6MWD: Six-min walk distance, 6MWSpeed: Six-min walk speed, 6MWW: six-min walk work, DSP: Distance saturation products, $\mathrm{SpO}_{2}$ : Oxygen saturation, EID: Exercise-induced desaturation. 

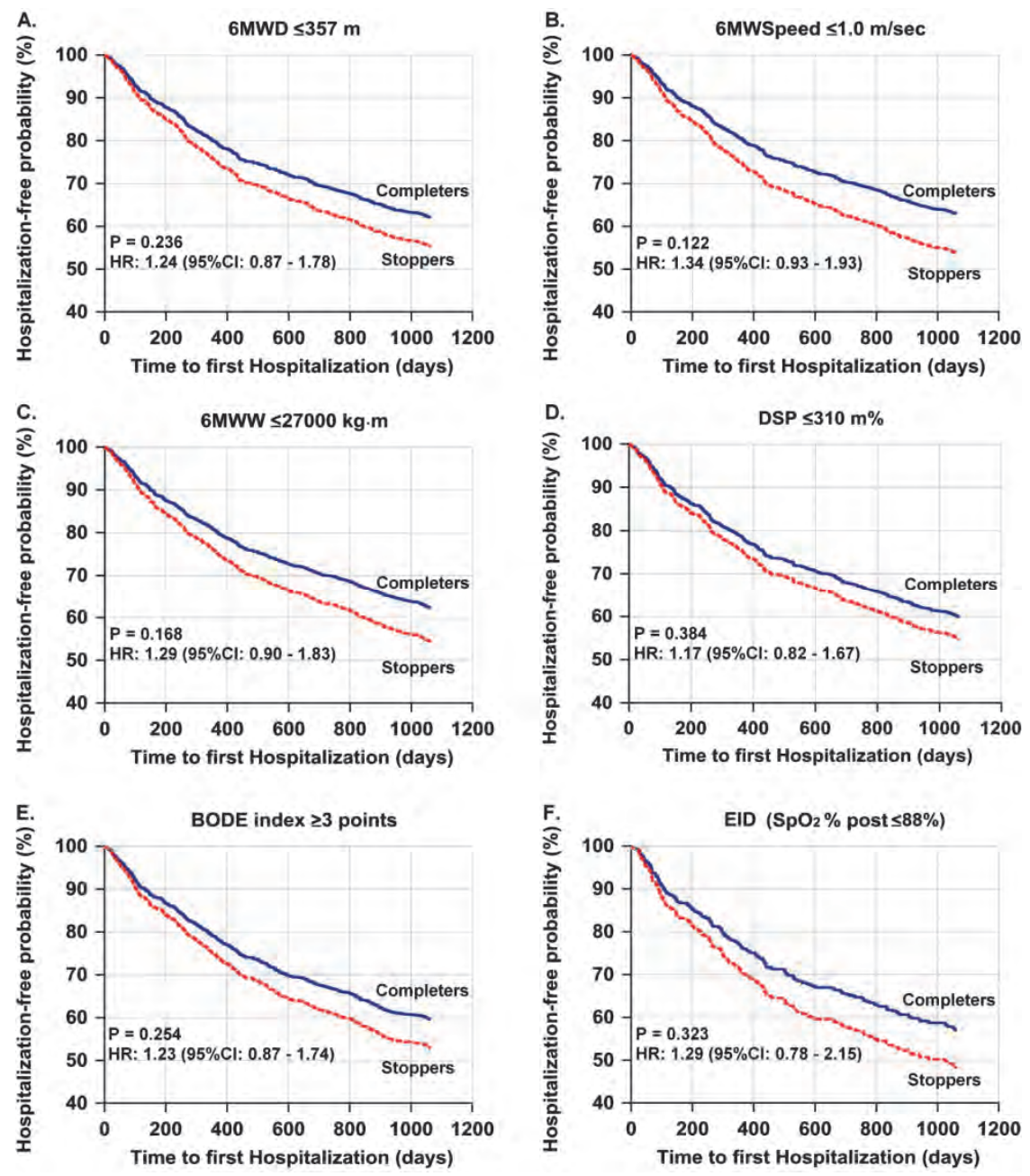

Figure S1. Kaplan-Meier curves for hospitalization referring to unintended stops of patients with impaired 6MWT-derived variables

Unintended stops (Completers/Stoppers) during the 6MWT were used for the estimation of hospitalizationfree probability (\%). Unadjusted hazard ratio (HR) and 95\% confidence intervals $(95 \% \mathrm{Cl})$ were calculated by Cox's proportional-hazard regression. Significance level was set at $P<0.05$. 


\section{Mortality}
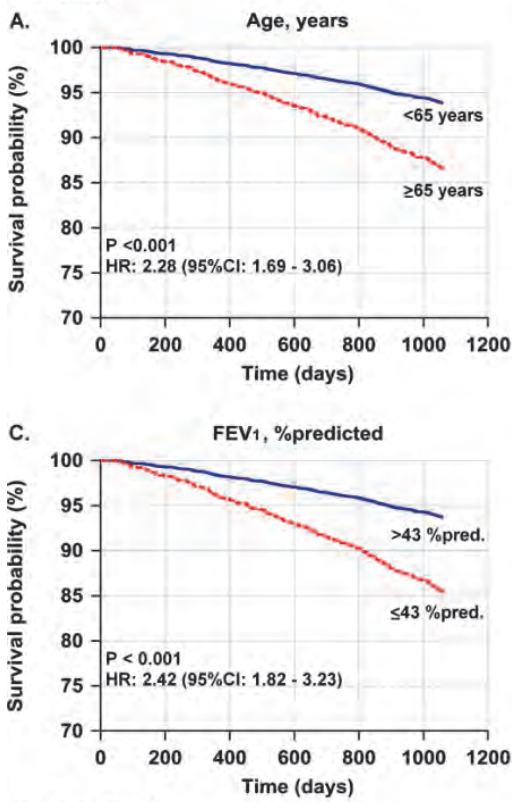

\section{Hospitalization}

E.

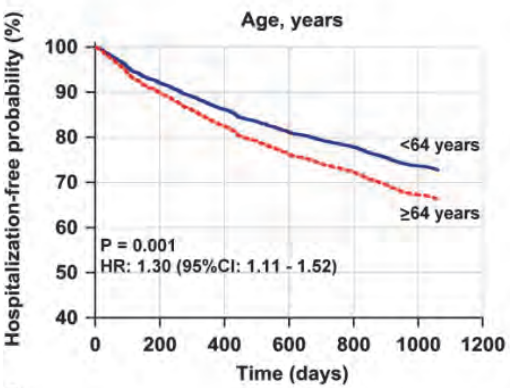

G.

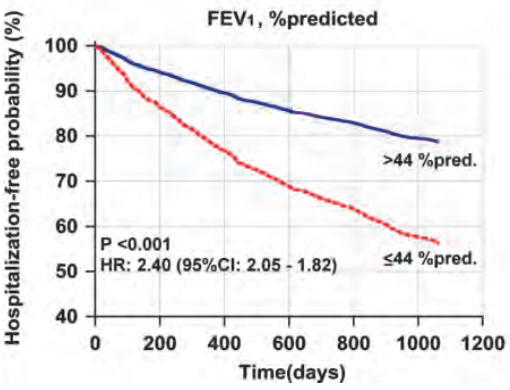

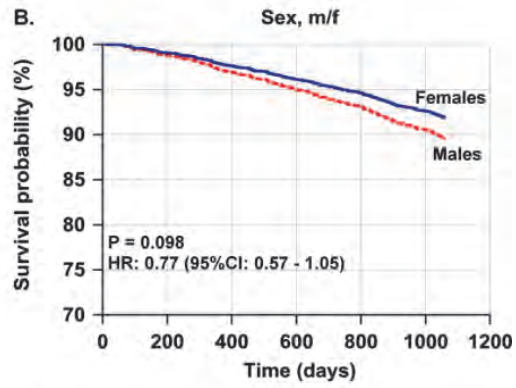

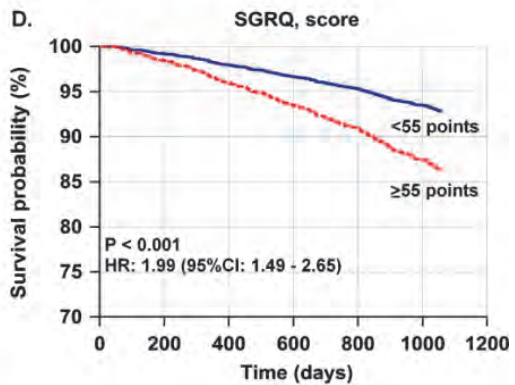

F.

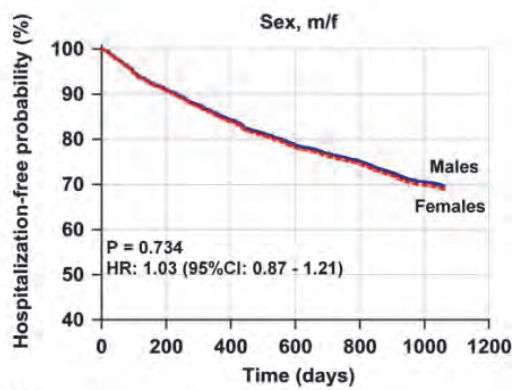

H.

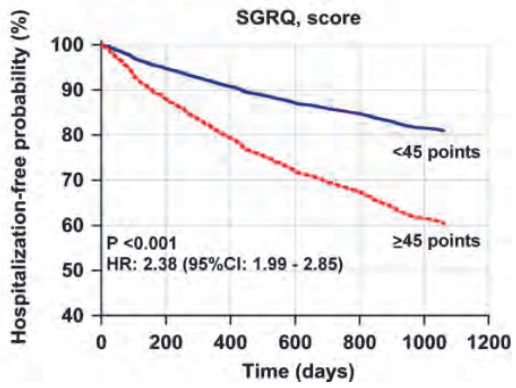

Figure S2. Kaplan-Meier curves for mortality and hospitalization according to clinical characteristics of patients with COPD

Certain thresholds of age, FEV $1, S G R Q$ and sex $(\mathrm{m} / \mathrm{f})$ were used for the estimation of survival and hospitalization-free probability (\%). Unadjusted hazard ratio (HR) and $95 \%$ confidence intervals $(95 \% \mathrm{Cl})$ were calculated by Cox's proportional-hazard regression. Significance level was set at $\mathrm{P}<0.05$. 


\section{Chapter}

\section{Determinants of exercise-induced oxygen desaturation including pulmonary emphysema in COPD: Results from the ECLIPSE study}

Vasileios Andrianopoulos, Bartolome R. Celli, Frits M.E. Franssen, Victor M. Pinto-Plata, Peter M.A. Calverley, Lowie E.G.W. Vanfleteren, Ioannis Vogiatzis, Jørgen Vestbo, Alvar Agusti, Per S. Bakke, Stephen I. Rennard, William MacNee, Ruth Tal-Singer, Julie C. Yates, Emiel F.M. Wouters, 


\section{Chapter 5}

\section{ABSTRACT}

\section{Background}

Exercise-induced oxygen desaturation (EID) is related to mortality in patients with chronic obstructive pulmonary disease (COPD). We investigated: (1) the prevalence of EID; (2) the relative-weight of several physiological determinants of EID including pulmonary emphysema, and (3) the relationship of EID with certain patients' clinical characteristics.

\section{Methods}

Data from 2050 COPD patients (age: $63.3 \pm 7.1$ years; $\mathrm{FEV}_{1}: 48.7 \pm 15.7 \%$ pred.) were analyzed. The occurrence of EID $\left(\mathrm{SpO}_{2}\right.$ post $\left.\leq 88 \%\right)$ at the six-minute walking test (6MWT) was investigated in association with emphysema quantified by computedtomography (QCT), and several clinical characteristics.

\section{Results}

435 patients (21\%) exhibited EID. Subjects with EID had more QCT-emphysema, lower exercise capacity and worse health-status (BODE, ADO indexes) compared to non-EID. Determinant of EID were obesity (BMI $\geq 30 \mathrm{~kg} / \mathrm{m}^{2}$ ), impaired $\mathrm{FEV}_{1}$ (44\%pred.), moderate or worse emphysema, and low $\mathrm{SpO}_{2}$ at rest (93\%). Linear regression indicated that each 1-point increase on the ADO-score independently elevates odds ratio (1.5fold) for EID.

\section{Conclusions}

About one in five COPD patients in the ECLIPSE cohort present EID. Advanced emphysema is associated with EID. In addition, obesity, severe airflow limitation, and low resting oxygen saturation increase the risk for EID. Patients with EID in GOLD stage II have higher odds to have moderate or worse emphysema compared those with EID in GOLD stage III-IV. Emphysematous patients with high ADO-score should be monitored for EID. 


\section{INTRODUCTION}

Exercise-induced oxygen desaturation (EID), as defined by fall in oxygen saturation to $88 \%$ or lower during exercise ${ }^{1,2}$, can occur in patients with Chronic Obstructive Pulmonary Disease (COPD), even in those without resting hypoxemia. ${ }^{3-5}$ Yet, their physiological determinants and clinical consequences are still subject of debate. In general, patients with EID are characterized by severe airflow limitation, low diffusing carbon monoxide capacity (DLCO), reduced resting arterial oxygenation ${ }^{6-9}$ and the presence of emphysema ${ }^{10-12}$ In addition, EID is also related to numerous variables that include the rate of lung function decline and health status ${ }^{13}$, physical activity ${ }^{14}$, and the outcomes of hospitalization as well as mortality. ${ }^{15,16}$ The determinants of EID, however, have not been validated in a large cohort of COPD patients followed up prospectively. The design and size of the ECLIPSE (Evaluation of COPD Longitudinally to Identify Predictive Surrogate Endpoints) study offers a unique opportunity to overcome these limitations. $^{17}$

Herein, we investigated in the ECLIPSE: (1) the prevalence of EID in COPD; (2) the relative weight of several physiological determinants of EID in COPD, particularly the severity of airflow limitation and the presence of emphysema as defined by quantitative computed tomography (QCT) analysis ${ }^{18-20}$ and, (3) the relationship of EID with certain patients' clinical characteristics.

\section{METHODS}

\section{Design and participants}

The ECLIPSE study (Clinicaltrials.gov identifier NCT00292552; GSK study code SC0104960) was a 3-year non-interventional longitudinal prospective study, as described elsewhere. ${ }^{17}$ In brief, individuals (age: $40-75$ years) were recruited to ECLIPSE if they had a smoking history of $\geq 10$ pack-years and a diagnosis of COPD. ${ }^{21}$ We excluded subjects with resting hypoxemia (as defined by $\mathrm{SpO}_{2} \%$ pre-walk $\leq 88 \%$ ) from this analysis. The ECLIPSE study was carried out in accordance with the Declaration of Helsinki and good clinical practice guidelines, and was approved by the ethics committees of participating centers. The Institutional Review Boards of all participating institutions approved the study and all participants provided written informed consent. Data from the ECLIPSE study have been published before ${ }^{16,22-24}$ but the current analysis, which is mostly focus on EID in association with the severity of emphysema, is novel and, therefore, complements previous reports. 


\section{Chapter 5}

\section{Subject characterization}

Demographic and physiological characteristics, level of dyspnea (using the modified Medical Research Council (mMRC) dyspnea scale $)^{25}$, measurements of lung function (post-bronchodilator spirometry and lung volumes), QCT-emphysema degrees, health status (using the St. George's Respiratory Questionnaire (SGRQ)) ${ }^{26}$, and data from 6MWT were used in this analysis. Fat mass and fat free mass were determined using bioelectrical impedance (Bodystat 1500). The fat free mass index (FFMI, $\mathrm{kg} / \mathrm{m}^{2}$ ) was calculated as the mass $(\mathrm{kg})$ divided by the squared height $\left(\mathrm{m}^{2}\right)$ and the body mass index $\left(\mathrm{BMI}, \mathrm{kg} / \mathrm{m}^{2}\right)$ was calculated as weight $(\mathrm{kg})$ divided by squared height $\left(\mathrm{m}^{2}\right)$. Furthermore, the following multidimensional COPD indices were calculated for the whole cohort; (1) the ADO index ${ }^{27}$, which includes age, dyspnea by the MRC, and $\mathrm{FEV}_{1} \%$, and (2) the BODE index ${ }^{28}$, which incorporates dyspnea by the $\mathrm{MRC}, \mathrm{BMI}, \mathrm{FEV}_{1} \%$, and exercise capacity as measured by six-minute walking distance (6MWD).

\section{CT imaging}

All subjects underwent a low-dose volumetric computed tomography (CT scan) at full inspiration. Scans were obtained using multi-detector row CT scanners (120 kVp, 40 $\mathrm{mAs}$ ) and were reconstructed using contiguous $1.00 \mathrm{~mm}$ or $1.25 \mathrm{~mm}$ slice thickness and an intermediate spatial frequency reconstruction algorithm. The radiation dose was estimated to be $1.67 \mathrm{mSv}$ per CT study or $5 \mathrm{mSv}$ for the entire ECLIPSE protocol. All CT scans were sent for evaluation at the central imaging unit at the University of British Columbia (Vancouver, BC, Canada). ${ }^{29}$ All CT scans were analyzed by the use of Pulmonary Workstation 2.0 software (VIDA Diagnostics, Coralville, IA, USA) and the severity of emphysema was quantified in CT scans (QCT) by two radiologists using the \% of lung pixels that were low attenuation areas (\%LAA) defined as being below 950 Hounsfield Units (density mask technique). ${ }^{18,20,30}$ The degree of QCT-emphysema was classified according to a 5-scale score as absent-trivial: <5\% (scores 0-1), mild: $5-25 \%$ (score 2), moderate: $25-50 \%$ (score 3), or severe to very severe: $>50 \%$ (scores 4-5). ${ }^{29}$

\section{Six-minute walk test (6MWT)}

The 6MWT was performed according to international recommendations. ${ }^{31}$ Briefly, participants were asked to walk indoors in a flat, straight, 30-m walking course supervised by a well-trained researcher. A practice 6MWT was not undertaken. Patients were encouraged using standardized encouragement every minute of the 6MWT. ${ }^{1}$ Resting (pre-walk) and post $\mathrm{SpO}_{2}$ were assessed using a pulse oxymeter with a finger probe. A modified Borg scale was used to quantify the levels of dyspnea and fatigue perceived by patients at the beginning and end of the test. All subjects included in the 
analysis were non-hypoxemic at rest $\left(\mathrm{SpO}_{2}\right.$ pre-walk $\left.\geq 89 \%\right)$. EID was defined as a fall of oxygen saturation to $\mathrm{SpO}_{2} \leq 88 \%$ at the end of the test. ${ }^{1,2}$

\section{Statistical analysis}

Results are reported as mean (standard deviation) or proportion (\%). The statistical significance of differences between groups was assessed by analysis of variance, paired T-tests and Chi-square, as appropriate. Effect sizes (Cohen's d) using absolute standardized differences among the comparisons between the non-EID and EID groups were calculated. Pearson's correlation coefficients were used to assess the bivariate relationship amongst patients' characteristics and the variables of $\mathrm{SpO}_{2} \%$ post-walk, $\triangle \mathrm{SpO}_{2} \%$ and QCT-defined emphysema. Linear regression and univariate and multivariate binary logistical regression models were used to assess individual predictors of EID. Receiver operating characteristics (ROC) curves were used to determine threshold values to predict EID. The Area Under the curve (AUC) was calculated by the trapezoidal rule and the confidence intervals of the AUC was computed by the method of DeLong. ${ }^{32}$ Two-sided level of significance was set at $P$ $<0.05$. Statistical analyses were carried out using MedCalc v.12, Sigmaplot v.12 and SPSS v.19.0.

\section{RESULTS}

\section{Subject characteristics}

A total of 2050 subjects with COPD were included in the study. As shown in Table 1, subjects generally had severe airflow limitation, low oxygen saturation $\left(\mathrm{SpO}_{2}\right.$ pre-walk), and were normal-weight to overweight. Of these, 1124 subjects (55\%) were assigned into GOLD III and IV while 622 subjects (33\%) were diagnosed with severe to very severe emphysema. Fifty-three percent (53\%) of the subjects complained of severe dyspnea ( $m M R C$ scale $\geq 2$ ), and $47 \%$ had severely impaired health status (SGRQ Total Score $\geq 50$ ). Thirty-six percent (36\%) were current smokers (Table 1).

\section{Determinants of EID}

Four hundred thirty-five subjects ( $n=435,21 \%$ ) exhibited EID while the prevalence of EID was higher across GOLD stages and the degrees of emphysema severity (Figure 1). Subjects with EID were older with more severe airflow limitation, lower FVC, and worse dyspnea (mMRC) as well as SGRQ total scores. Also, they were more commonly exsmokers compared those without EID (non-EID; Table 1). Subjects with EID had a shorter walk distance (6MWD), lower pre-walk $\mathrm{SpO}_{2}$ and higher pre/post-walk heart 


\section{Chapter 5}

rate. In addition, EID subjects had greater increase of heart rate from baseline $(\Delta H R)$ and more intense dyspnea at exertion compared to non-EID (Table 2). Moreover, those with EID had higher scores in the $\mathrm{ADO}^{27}$ and the $\mathrm{BODE}^{28}$ indices compared to non-EID subjects (Table 2).

The effect of QCT-emphysema and the severity of airflow limitation on the lowest values of oxygen saturation levels, which defined the $\operatorname{EID}\left(\mathrm{SpO}_{2}\right.$ post $\left.\leq 88 \%\right)$ in the $6 \mathrm{MWT}$, varied widely across the disease severity (Figure 2). Both, severity of airflow limitation and the extent of emphysema had a negative impact on the $\mathrm{SpO}_{2}$ levels at the end of 6MWT. Severe to very severe emphysema in advanced airflow limitation is highly related to EID. On the other hand, the odds for patients with EID to have moderate or worse emphysema were greater in patients with GOLD II compared to GOLD III-IV (ORs 5.22, 2.11 and 1.48, respectively; Figures 1 and 2).

Despite the large variability in the proportion of patients who presented EID across the disease severity, the overall change in oxygen saturation $\left(\Delta \mathrm{SpO}_{2}\right)$ associated with the severity of emphysema was relatively consistent (Figure 3).

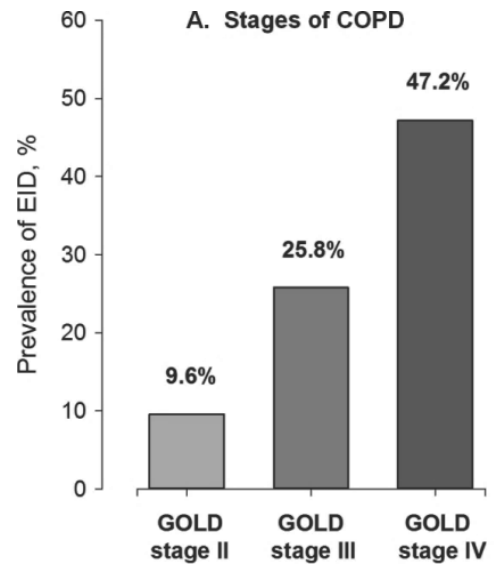

C. Overall

Figure 1. Prevalence of EID in COPD according to GOLD stages and pulmonary emphysema EID progressively increased across the GOLD stages and the degrees of pulmonary emphysema in COPD. A total of 435 subjects (21\%) in the ECLIPSE cohort exhibited EID. 
Determinants of EID including pulmonary emphysema in COPD: the ECLIPSE study

Table 1. Baseline characteristics of COPD patients categorized by the prevalence of EID

\begin{tabular}{|c|c|c|c|c|c|}
\hline Characteristics & $\begin{array}{l}\text { All Subjects } \\
(n=2050)\end{array}$ & $\begin{array}{l}\text { Non-EID } \\
(n=1615,79 \%)\end{array}$ & $\begin{array}{l}\text { EID } \\
(n=435,21 \%)\end{array}$ & P Value & $\begin{array}{l}\text { Cohen's } \\
\text { d }\end{array}$ \\
\hline Women, \#, (\%) & $710(35)$ & $544(34)$ & $166(38)$ & & \\
\hline Age, years & $63.3 \pm 7.1$ & $63.1 \pm 7.2$ & $64.1 \pm 6.8$ & 0.012 & 0.143 \\
\hline $\mathrm{BMI}, \mathrm{kg} / \mathrm{m}^{2}$ & $26.5 \pm 5.6$ & $26.5 \pm 5.5$ & $26.7 \pm 5.8$ & NS & 0.035 \\
\hline Underweight & $98(5)$ & $81(5)$ & $17(4)$ & & \\
\hline Normal range & $751(37)$ & $591(36)$ & $160(37)$ & & \\
\hline Overweight & $743(36)$ & $594(37)$ & 149(34) & & \\
\hline Obese & $458(22)$ & $349(22)$ & $109(25)$ & $N S^{\#}$ & 0.928 \\
\hline FFMI, $\mathrm{kg} / \mathrm{m}^{2}$ & $17.8 \pm 3.4$ & $17.8 \pm 3.5$ & $17.6 \pm 3.4$ & NS & 0.058 \\
\hline $\mathrm{FEV}_{1}, \%$ predicted & $48.7 \pm 15.7$ & $51.2 \pm 15.2$ & $39.5 \pm 13.9$ & $<0.001$ & 0.803 \\
\hline FVC, \% predicted & $80.0 \pm 19.8$ & $81.2 \pm 19.2$ & $75.4 \pm 21.0$ & $<0.001$ & 0.288 \\
\hline $\mathrm{FEV}_{1} / \mathrm{FVC}, \%$ & $44.7 \pm 11.4$ & $46.2 \pm 11.0$ & 39.111 .1 & $<0.001$ & 0.643 \\
\hline \multicolumn{6}{|l|}{ GOLD Classification ${ }^{\S}$} \\
\hline Stage II & $925(45)$ & $836(52)$ & $89(20)$ & & \\
\hline Stage III & $861(42)$ & 639(39) & $222(51)$ & & \\
\hline Stage IV & $263(13)$ & 139(9) & $124(29)$ & $<0.001^{\#}$ & 0.114 \\
\hline \multicolumn{6}{|l|}{ Emphysema extent ${ }^{\S \S}$} \\
\hline$<5 \%$ [absent-trivial] & $478(26)$ & $431(30)$ & $47(11)$ & & \\
\hline $5-25 \%[$ Mild] & $406(22)$ & $356(24)$ & $50(12)$ & & \\
\hline 25-50\% [Moderate] & $361(19)$ & $293(20)$ & $68(17)$ & & \\
\hline$>50 \%$ [Severe-very severe] & $622(33)$ & $375(26)$ & $247(60)$ & $<0.001^{\#}$ & 0.403 \\
\hline mMRC, Dyspnea grade & $1.7 \pm 1.0$ & $1.6 \pm 1.0$ & $2.0 \pm 1.0$ & $<0.001$ & 0.400 \\
\hline$\geq 2$ & $1062(53)$ & $775(48)$ & $287(66)$ & $<0.001^{\#}$ & 0.835 \\
\hline SGRQ-C Total Score & $47.8 \pm 18.1$ & $46.5 \pm 18.5$ & $52.6 \pm 15.6$ & $<0.001$ & 0.356 \\
\hline$\geq 50$ points, $n(\%)$ & $934(47)$ & $700(43)$ & $234(54)$ & $<0.001^{\#}$ & 0.893 \\
\hline Current Smokers, n (\%) & $742(36)$ & $639(40)$ & $103(24)$ & $<0.001^{\#}$ & 1.171 \\
\hline
\end{tabular}

Abbreviations: BMI: Body Mass Index, FFMI: Fat-Free Mass Index, FEV 1 : Force Expiratory Volume at 1 sec, FVC: Force Vital Capacity, mMRC: modified Medical Research Council Dyspnea Scale, SGRQ-C: St. George's Respiratory Questionnaire for COPD (40-item version).Values expressed as mean \pm SD. ${ }^{5}$ One patients had a confirmed diagnosis for COPD but could not be assigned to a GOLD stage. ${ }^{\S \xi}$ In total 183 patient had undefined extent of emphysema (\% valid percent). Hash tag (\#) denotes statistical significance detected by Pearson Chisquare analysis Significance level was set at $\mathrm{P}<0.05$. 


\section{Chapter 5}

Table 2. Six-Minute Walk Test characteristics and multi-dimensional indices categorized by the prevalence of EID

\begin{tabular}{|c|c|c|c|c|c|}
\hline Characteristics & $\begin{array}{l}\text { All Subjects } \\
(n=2050)\end{array}$ & $\begin{array}{l}\text { Non-EID } \\
(n=1615,79 \%)\end{array}$ & $\begin{array}{l}\text { EID } \\
(n=435,21 \%)\end{array}$ & $\begin{array}{l}\mathrm{P} \\
\text { Value }\end{array}$ & $\begin{array}{l}\text { Cohen's } \\
\text { d }\end{array}$ \\
\hline $6 \mathrm{MWD}, \mathrm{m}, \pm \mathrm{SD}$ & $372 \pm 121$ & $382 \pm 119$ & $334 \pm 121$ & $<0.001$ & 0.400 \\
\hline 6MWD, \% predicted & $64 \pm 21$ & $66 \pm 20$ & $58 \pm 21$ & $<0.001$ & 0.390 \\
\hline $\mathrm{SpO}_{2}, \%$ pre & $94.7 \pm 2.2$ & $95.1 \pm 2.0$ & $93.1 \pm 2.3$ & $<0.001$ & 0.928 \\
\hline $\mathrm{SpO}_{2}, \%$ post & $91.8 \pm 5.0$ & $93.9 \pm 2.6$ & $84.1 \pm 4.3$ & $<0.001$ & 2.758 \\
\hline$\Delta \mathrm{SpO}_{2}, \%$ & $-2.9 \pm 4.3$ & $-1.2 \pm 2.4$ & $-9.0 \pm 4.8$ & $<0.001$ & 2.055 \\
\hline HR pre, b/m & $82 \pm 14$ & $81 \pm 14$ & $85 \pm 14$ & $<0.001$ & 0.286 \\
\hline HR post, b/m & $100 \pm 18$ & $98 \pm 17$ & $108 \pm 19$ & $<0.001$ & 0.555 \\
\hline$\Delta \mathrm{HR}, \mathrm{b} / \mathrm{m}$ & $18 \pm 14$ & $17 \pm 13$ & $23 \pm 16$ & $<0.001$ & 0.412 \\
\hline \multicolumn{6}{|l|}{ Borg Scale } \\
\hline Dyspnea, pre & $1.6 \pm 1.8$ & $1.5 \pm 1.8$ & $1.7 \pm 1.9$ & NS & 0.108 \\
\hline Dyspnea, post & $3.9 \pm 2.4$ & $3.6 \pm 2.3$ & $4.7 \pm 2.3$ & $<0.001$ & 0.478 \\
\hline$\Delta$ Dyspnea, $[\Delta]$ & $2.3 \pm 2.0$ & $2.1 \pm 1.9$ & $3.0 \pm 2.2$ & $<0.001$ & 0.438 \\
\hline Fatigue, pre & $1.3 \pm 1.8$ & $1.3 \pm 1.8$ & $1.3 \pm 1.8$ & NS & 0.000 \\
\hline Fatigue, post & $2.7 \pm 2.5$ & $2.6 \pm 2.5$ & $2.7 \pm 2.5$ & NS & 0.040 \\
\hline$\Delta$ Fatigue, $[\Delta]$ & $1.4 \pm 1.9$ & $1.4 \pm 1.8$ & $1.4 \pm 1.9$ & NS & 0.000 \\
\hline ADO index, score & $3.7 \pm 1.5$ & $3.5 \pm 1.5$ & $4.4 \pm 1.4$ & $<0.001$ & 0.620 \\
\hline$\geq 6$ points, $n,(\%)$ & $245(12)$ & $148(9)$ & $97(22)$ & $<0.001^{\#}$ & 0.408 \\
\hline BODE index, score & $3.1 \pm 2.1$ & $2.8 \pm 2.0$ & $4.2 \pm 2.1$ & $<0.001$ & 0.683 \\
\hline$\geq 6$ points, $n,(\%)$ & $456(22)$ & $286(18)$ & $170(39)$ & $<0.001^{\#}$ & 0.493 \\
\hline
\end{tabular}

Abbreviations: BMI: Body Mass Index, FFMI: Fat-Free Mass Index, FEV 1 : Force Expiratory Volume at 1 sec, FVC: Force Vital Capacity, 6MWD: Six-min walk distance, SpO2: Oxygen saturation, HR: Heart rate, ADO index: "Age, Dyspnea, and airflow Obstruction" - index, BODE index: "Body-mass index, airflow Obstruction, Dyspnea, and Exercise" - index, EID: Exercise-induced oxygen desaturation ( $\mathrm{SpO}_{2}$ post 88\%). Values expressed as mean \pm SD unless specified otherwise. Hash tag (\#) denotes statistical significance detected by Pearson Chisquare analysis. Significance level was set at $\mathrm{P}<0.05$.

\section{Multivariate correlates of EID}

Using receiver operating characteristics (ROC) curves, the threshold values with the best specificity and sensitivity to predict EID were determined for age ( $\geq 60$ years), sex (female), BMI ( $\left.\geq 30 \mathrm{~kg} / \mathrm{m}^{2}\right), \mathrm{FEV}_{1}$ ( $\leq 44 \%$ pred.), QCT-emphysema (moderate to very severe), resting oxygen saturation $\left(\mathrm{SpO}_{2}\right.$ pre-walk $\left.\leq 93 \%\right)$ and $\mathrm{ADO}$ index (ADO score $\geq 6$ points; Supplementary table S1). Univariate logistical regression analysis revealed that older age and impaired levels of $\mathrm{FEV}_{1}, \mathrm{QCT}$-emphysema, and $\mathrm{SpO}_{2}$ pre-walk were independent predictors of EID. Multivariate logistical regression model showed that only impaired levels of $\mathrm{FEV}_{1}, \mathrm{QCT}$-emphysema, and $\mathrm{SpO}_{2}$ pre-walk remained significant ( $p \leq 0.016)$. Interestingly, obesity $\left(\mathrm{BMI} \geq 30 \mathrm{~kg} / \mathrm{m}^{2}\right.$ ) reached the statistical significance 
( $p<0.003$ ) for contributing EID only within this multivariate-adjusted model and not as independent determinant (Table 3).

A combination of certain clinical characteristics including obesity, moderate to very severe emphysema, severe airflow limitation ( $\mathrm{FEV}_{1} \leq 44 \%$ pred.), and resting $\mathrm{SpO}_{2} \leq 93 \%$ revealed that the $81 \%$ of the subjects with all of these characteristics exhibited EID (Figure 4).

\section{Relationship of ADO score with EID}

The presence of EID was related to higher ADO index ${ }^{27}$ scores in EID subjects with GOLD II-III and across all the degrees of emphysema compared to those with non-EID (Supplementary figure 1). For each 1-point increase on the ADO score, the odds ratio for EID was independently increased by 1.5 fold while $40 \%$ of subjects with ADO $\geq 6$ points had EID (Supplementary table S2).

Table 3. Logistic regression: Determinants of EID in COPD patients

\begin{tabular}{|c|c|c|c|c|c|c|}
\hline Determinants & $\begin{array}{l}\text { Subjects } \\
\mathrm{n}\end{array}$ & $\begin{array}{l}\text { EID } \\
n,(\%)\end{array}$ & $\begin{array}{l}\text { Crude OR } \\
(95 \% \mathrm{Cl})\end{array}$ & $\begin{array}{l}P \\
\text { value }\end{array}$ & $\begin{array}{l}\text { Adjust OR } \\
(95 \% \mathrm{Cl})\end{array}$ & $\begin{array}{l}P \\
\text { value }\end{array}$ \\
\hline \multicolumn{7}{|l|}{ Age } \\
\hline$<60$ years & 594 & $106(18)$ & 1 & & 1 & \\
\hline$\geq 60$ years & 1456 & $329(23)$ & $1.34(1.05-1.71)$ & 0.017 & $1.21(0.91-1.61)$ & NS \\
\hline \multicolumn{7}{|l|}{ Sex } \\
\hline Male & 1340 & $269(20)$ & 1 & & 1 & \\
\hline Female & 710 & $166(23)$ & $1.22(0.98-1.51)$ & NS & $1.30(1.00-1.68)$ & NS \\
\hline \multicolumn{7}{|l|}{ BMI } \\
\hline$<30 \mathrm{~kg} / \mathrm{m}^{2}$ & 1592 & $326(20)$ & 1 & & 1 & \\
\hline$\geq 30 \mathrm{~kg} / \mathrm{m}^{2}$ & 458 & $109(24)$ & $1.21(0.95-1.55)$ & NS & $1.57(1.15-2.14)$ & 0.005 \\
\hline \multicolumn{7}{|l|}{$\mathrm{FEV}_{1}$} \\
\hline$>44$ \%predicted & 1198 & $141(12)$ & 1 & & 1 & \\
\hline$\leq 44 \%$ predicted & 851 & $294(35)$ & $3.96(3.16-4.96)$ & $<0.001$ & $3.14(2.42-4.07)$ & $<0.001$ \\
\hline \multicolumn{7}{|l|}{ Emphysema } \\
\hline Absent to mild & 884 & $97(11)$ & 1 & & 1 & \\
\hline $\begin{array}{l}\text { Moderate to very } \\
\text { severe }\end{array}$ & 983 & $315(32)$ & $3.83(2.98-4.91)$ & $<0.001$ & $3.37(2.52-4.51)$ & $<0.001$ \\
\hline \multicolumn{7}{|l|}{$\mathrm{SpO}_{2} \%$ pre-walk } \\
\hline$>93 \%$ & 1506 & $194(13)$ & 1 & & 1 & \\
\hline$\leq 93 \%$ & 544 & $241(44)$ & $5.38(4.29-6.75)$ & $<0.001$ & 4.75 (3.69-6.13) & $<0.001$ \\
\hline
\end{tabular}

Abbreviations: BMI: Body Mass Index, $\mathrm{FEV}_{1}$ : Force Expiratory Volume at $1 \mathrm{sec}, \mathrm{SpO}_{2}$ : Oxygen saturation. Multivariate-adjusted model including all the determinants of EID. A multivariate-adjusted regression analysis; all the determinants of EID (age, sex, $\mathrm{BMI}_{1} \mathrm{FEV}_{1}$, emphysema, and $\mathrm{SpO}_{2}$ pre-walk) were included in the multivariate model. Significance level was set at $\mathrm{P}<0.05$. 


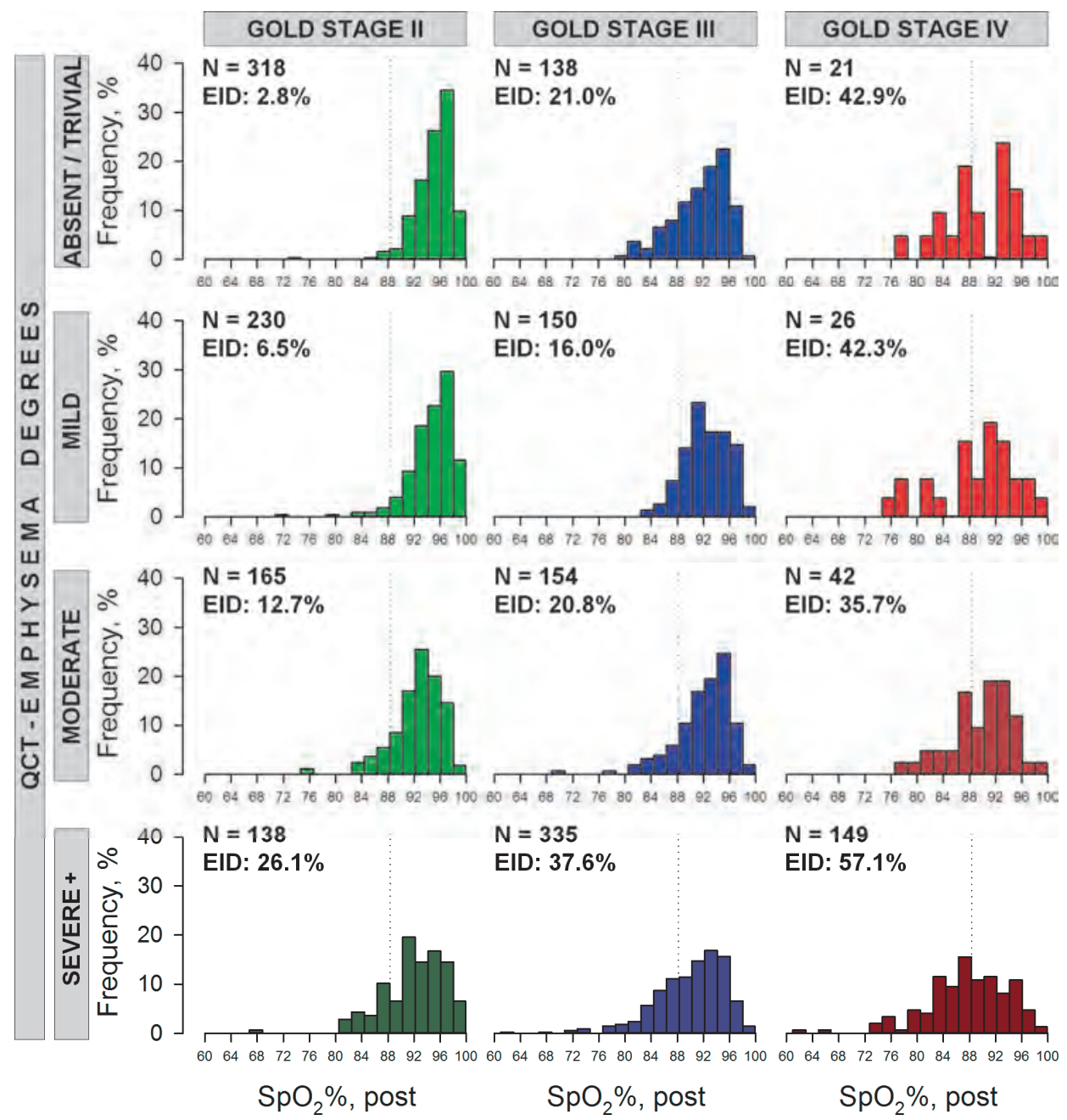

Figure 2. Frequency distribution of post-walk $\mathrm{SpO}_{2}$ and EID (88\%) stratified by the GOLD stages and the degrees of QCT-emphysema

The dot line marks the threshold value for the presence of EID $\left(\mathrm{SpO}_{2}\right.$ post-walk $\left.88 \%\right)$. 


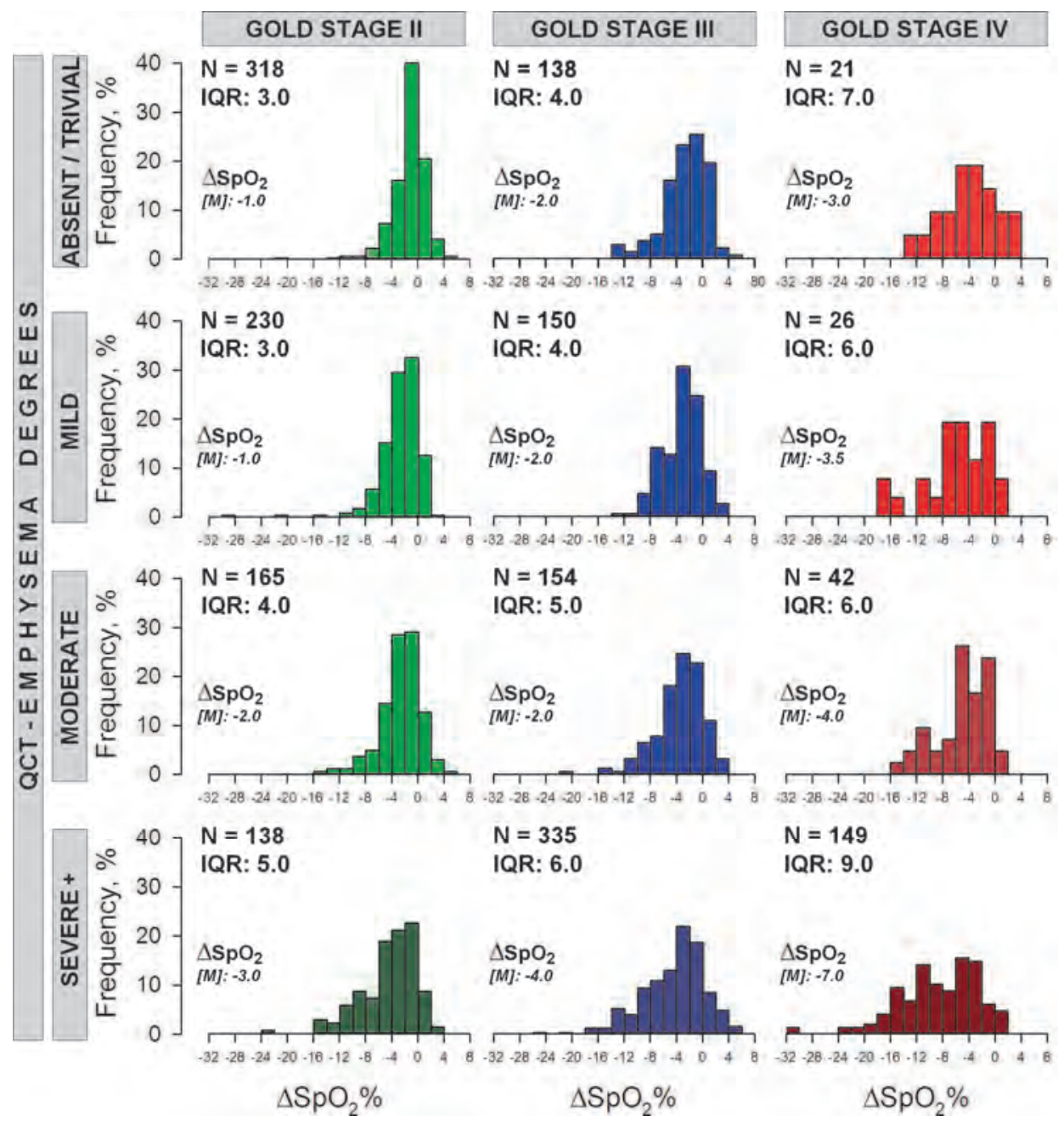

Figure 3. Frequency distribution of the changes in oxygen saturation $\left(\Delta \mathrm{SpO}_{2}\right)$, as occurred from baseline to post-walk values, stratified by the GOLD stages and the degrees of QCT emphysema

The interquartile range $(I \mathrm{QR})$ and the median changes in oxygen saturation $\left(\Delta \mathrm{SpO}_{2}[\mathrm{M}]\right)$ were calculated. 


\section{Chapter 5}

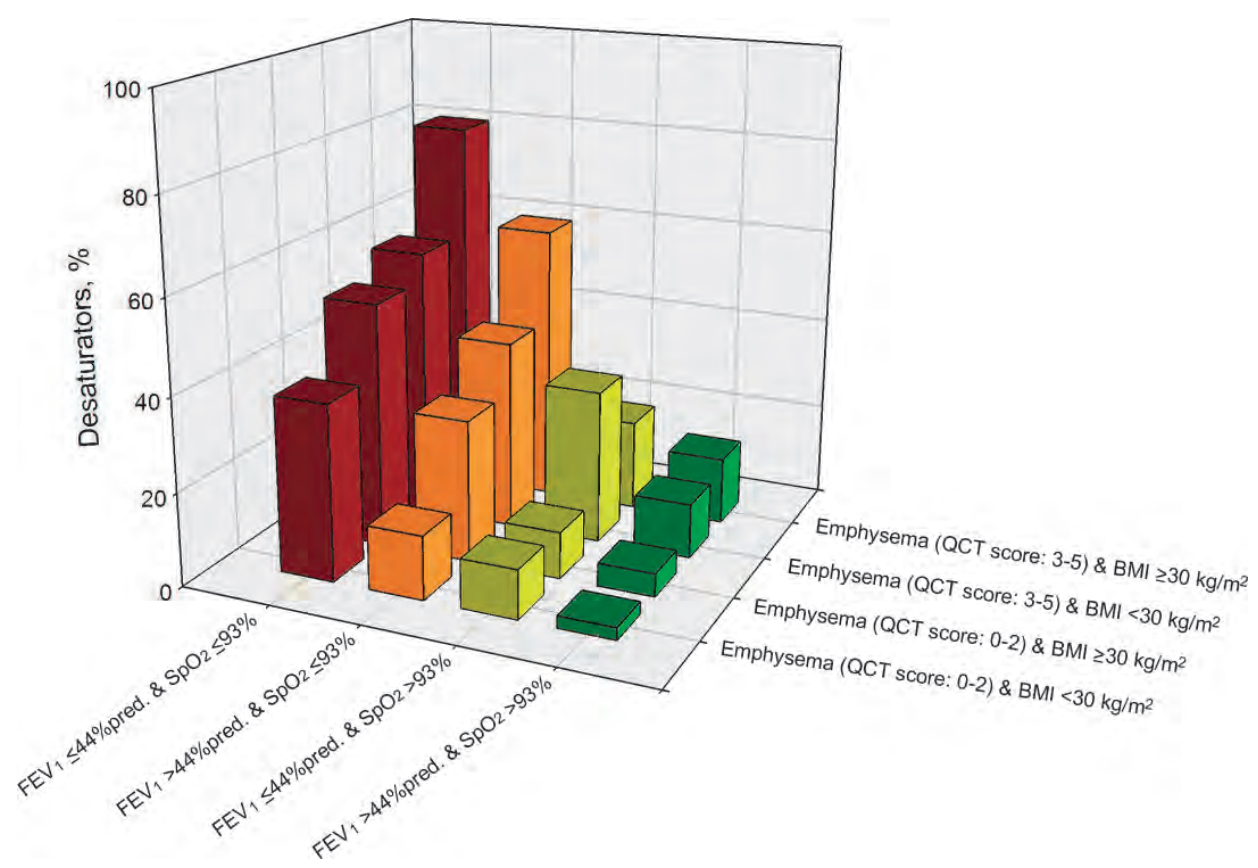

Figure 4. Combination of certain lung function characteristics and resting oxygen saturation applied for the prediction of EID in patients with COPD

A combination of certain lung function characteristics and resting oxygen saturation for the prediction of EID revealed that the highest proportion of patients (81\%) who exhibit EID are characterized by moderate to very severe emphysema (QCT score 3-5), obesity, impaired $\mathrm{FEV}_{1}$ and low resting $\mathrm{SpO}_{2}$ pre-walk values.

\section{DISCUSSION}

This study provides three major observations: (1) about 20\% of COPD patients included in the ECLIPSE cohort exhibited EID; (2) emphysema, severity of airflow limitation, arterial oxygen saturation at rest and obesity are associated with EID; and (3) a high ADO index is predictive of EID.

It has been previously suggested that the presence of emphysema may determine EID in COPD. ${ }^{11}$ However; this is the first time that the role of emphysema was investigated in a large well-characterized COPD population (ECLIPSE cohort). In this study, emphysema in association with other determinants, such as obesity (BMI $\geq 30 \mathrm{~kg} / \mathrm{m}^{2}$ ), have a prognostic value in the prediction of EID. Moreover, the relationship of the ADO score with EID was a novel investigation. 


\section{Interpretation of results}

In current study, the prevalence of EID was increased across the GOLD stages (Figure 1). The severity of airflow limitation seems to contribute to the occurrence of EID along with than the grades of QCT-emphysema (Figure 2). In addition, the occurrence of EID in subjects with COLD II may indicate the presence of emphysema according to the findings of this study (Figure 2). However, this was not true in GOLD III and IV as both airflow limitation and emphysema likely contribute to EID.

Impaired oxygen transport and utilization is a common consequence in both airway limitation and pulmonary emphysema, and can lead to ventilation/perfusion (V/Q) mismatch resulting in EID. Airflow limitation often leads to air-trapping and dynamic hyperinflation, which result in higher work of breathing due to high inspiratory threshold load and is associated to increased oxygen consumption. ${ }^{33-35}$ On the other hand, emphysema is linked to decreased alveolar surface area and loss of elastic recoil of the lungs. ${ }^{36,37}$ In addition, the presence of large airway disease (airway thickening) may also contribute to $\mathrm{V} / \mathrm{Q}$ inequality during exercise and to EID. ${ }^{38,39}$ In current study, the negative influence of very low FEV 1 (GOLD III-IV) ${ }^{5,39}$ and severe emphysema on oxygen saturation levels was exclusively taken into account showing that very low $\mathrm{FEV}_{1}$, might be overlapping with the contribution of severe emphysema on the occurrence of EID. Nevertheless, correlation analysis of this study indicated that emphysema as well as airflow limitation similarly correlated to the post-walk $\mathrm{SpO}_{2}(r=-0.35$ and $r=0.38$, respectively, $p<0.001$; Supplementary table S3) showing that severity of emphysema can be also a major determinant of EID, especially in moderate COPD. Specifically, severe to very severe emphysema seems to have a significant association with EID rather than the lower degrees of emphysema in patients with COPD.

In this study, an important finding is that obesity was associated with the presence of EID in COPD. Previously, the association of lower $\mathrm{SpO}_{2}$ with obesity has been reported for 871 emergency department patients and hospital workers. ${ }^{40}$ Kapur and colleagues have also demonstrated that obesity is associated with a lower resting oxygen saturation examining a large cohort of 2252 elderly subjects from the Cardiovascular Health Study. ${ }^{41}$ In COPD, one study confirms a correlation between COPD severity with $\mathrm{BMI}$ and oxygen saturation using measurements of $\mathrm{SpO}_{2}$ values at rest. $^{42}$ To the best of our knowledge, our findings revealed for the first time a potential relationship between obesity and EID during the 6MWT in subjects with COPD. However, the effect of obesity on the occurrence of EID is likely depended from the degree of emphysema. The direct physiological mechanism underlying the effect of obesity on EID is complex; however, obesity and EID could be related to low baseline $\mathrm{SpO}_{2}$, which can be considered as one of the major determinant of EID. ${ }^{5,8}$

In this study, we also investigated the relationship of the ADO index ${ }^{27}$ and EID. Previously, the prognostic value of the ADO index regarding the risk of exacerbation and 
death has been reported, however, ADO prognostic value of EID has never been investigated. ${ }^{43,44}$ Multidimensional indices for the prognosis of oxygen desaturation have been proposed by Cutaia and colleagues ${ }^{45}$ who suggested that patients with an increased BODE index ${ }^{28}$ ( $\geq 7$ points) should be evaluated for oxygen desaturation during daily activities. ${ }^{45}$ In our analysis, the selection of the ADO index ${ }^{27}$ for screening EID was based on the fact that exercise or a 6MWT is not required in order to record a score for this clinical index. Patients with high score of $A D O$ index $^{27}$ ( $\geq 6$ points) presented a greater occurrence of EID (40\% vs 19\%; $p<0.001$; Table 2). This can be attributed to severe airflow limitation and/or extended emphysema which was common in those patients with high ADO scores. Subjects with EID in GOLD II-III had higher ADO scores compared to non-EID, but this was not true in GOLD IV, while ADO scores were also greater in subjects with EID across the emphysema severity (Supplementary figure S1). However, we did not include the ADO index in our multivariate logistical model as age and $\mathrm{FEV}_{1}$, which are the main elements of the ADO index, were already included as independent determinants in this statistical analysis.

In our multivariate logistical regression model, obese subjects with an impaired $\mathrm{FEV}_{1}$ $(\leq 44 \%)$, moderate to very severe emphysema, and low pre-walk $\mathrm{SpO}_{2}(\leq 93 \%)$ have higher odds for EID (Table 3 and Figure 4). As it was expected, the role of advanced emphysema in EID was critical and therefore emphysematous patients should be screened for EID, especially those with high ADO scores.

\section{Strengths and weaknesses}

There are certain limitations to our study. First, the 6MWT screening was performed only once at baseline, while the 2014 ERS/ATS technical standard for field walking tests suggests the conduct of two 6MWT. ${ }^{1}$ Second, EID was defined based on the $\mathrm{SpO}_{2}$ at the end of the test $\left(\mathrm{SpO}_{2}\right.$ post) and not the $\mathrm{SpO}_{2}$ nadir which can lead to an underestimation of the patients who exhibit EID during the 6MWT. Even though $\mathrm{SpO}_{2}$ post and the $\mathrm{SpO}_{2}$ nadir for most patients with chronic respiratory disease are relatively similar during the $6 \mathrm{MWT}^{46}$, the use of $\mathrm{SpO}_{2}$ nadir would be optimal absent the biases. Moreover, the definition of EID did not include the magnitude of $\mathrm{SpO}_{2}$ degrease, however, only 33 of the patients with $\mathrm{EID}$ (7.5\%) had a $\mathrm{SpO}_{2}$ decline less than $4 \%$. Third, blood gas analysis and diffusion capacity (DLCO) were not available in our dataset, which could provide further physiological evidence for the presence of EID. Forth, additional factors such as quantified air-trapping and airway wall thickness ${ }^{39}$ did not evaluate for potential contribution on EID. Despite these limitations, this study contributes with important information about emphysema, airflow limitation and obesity as determinants of EID and the use ADO index ${ }^{27}$ as an additional clinical tool for screening EID in patients with COPD. Our findings may help clinicians to better understand the relationship of emphysema with EID and predict more accurately the EID. 


\section{CONCLUSIONS}

In summary, EID occurs in about $20 \%$ of COPD patients. Several physiological determinants including presence of advanced emphysema, severe airflow limitation, low oxygen saturation at rest, and obesity, contribute to EID which seems to be captured by high ADO index ( $\geq 6$ points) in GOLD stage II-III. Also, patients with EID in GOLD stage II have higher odds of having moderate or worse emphysema compared those with EID in GOLD stage III-IV. Emphysematous patients with high ADO score should be monitored for EID upon embarking on a clinical exercise training program. 


\section{REFERENCES}

1. Holland AE, Spruit MA, Troosters T, et al. An official European Respiratory Society/American Thoracic Society technical standard: field walking tests in chronic respiratory disease. Eur Respir J. 2014; 44: 1428-46.

2. Singh SJ, Puhan MA, Andrianopoulos V, et al. An official systematic review of the European Respiratory Society/American Thoracic Society: measurement properties of field walking tests in chronic respiratory disease. Eur Respir J. 2014; 44: 1447-78.

3. Mak VH, Bugler JR, Roberts CM and Spiro SG. Effect of arterial oxygen desaturation on six minute walk distance, perceived effort, and perceived breathlessness in patients with airflow limitation. Thorax. 1993; 48: $33-8$.

4. Jenkins S and Cecins N. Six-minute walk test: observed adverse events and oxygen desaturation in a large cohort of patients with chronic lung disease. Intern Med J. 2011; 41: 416-22.

5. Andrianopoulos V, Franssen FM, Peeters JP, et al. Exercise-induced oxygen desaturation in COPD patients without resting hypoxemia. Respir Physiol Neurobiol. 2014; 190: 40-6.

6. Kelley MA, Panettieri RA, Jr. and Krupinski AV. Resting single-breath diffusing capacity as a screening test for exercise-induced hypoxemia. Am J Med. 1986; 80: 807-12.

7. Owens GR, Rogers RM, Pennock BE and Levin D. The diffusing capacity as a predictor of arterial oxygen desaturation during exercise in patients with chronic obstructive pulmonary disease. N Eng/ J Med. 1984; 310: 1218-21.

8. Knower MT, Dunagan DP, Adair NE and Chin R, Jr. Baseline oxygen saturation predicts exercise desaturation below prescription threshold in patients with chronic obstructive pulmonary disease. Arch Intern Med. 2001; 161: 732-6.

9. Crisafulli E, lattoni A, Venturelli E, et al. Predicting Walking-Induced Oxygen Desaturations in Copd Patients: A Statistical Model. Respir Care. 2013.

10. Gould GA, Redpath AT, Ryan M, et al. Lung CT density correlates with measurements of airflow limitation and the diffusing capacity. Eur Respir J. 1991; 4: 141-6.

11. Taguchi O, Gabazza EC, Yoshida M, et al. CT scores of emphysema and oxygen desaturation during lowgrade exercise in patients with emphysema. Acta Radiol. 2000; 41: 196-7.

12. Kim C, Park YB, Seo JB, Oh Y-M and Lee S-D. The CT emphysema index is a predictor for exertional desaturation in COPD patients without resting hypoxemia. Eur Respir J. 2012; 40.

13. Kim C, Seo JB, Lee SM, et al. Exertional Desaturation as a Predictor of Rapid Lung Function Decline in COPD. Respiration. 2013; 86: 109-16.

14. Vogiatzis I, Zakynthinos $\mathrm{G}$ and Andrianopoulos V. Mechanisms of physical activity limitation in chronic lung diseases. Pulm Med. 2012; 2012: 634761.

15. Casanova C, Cote C, Marin JM, et al. Distance and oxygen desaturation during the 6-min walk test as predictors of long-term mortality in patients with COPD. Chest. 2008; 134: 746-52.

16. Andrianopoulos V, Wouters EF, Pinto-Plata VM, et al. Prognostic value of variables derived from the sixminute walk test in patients with COPD: Results from the ECLIPSE study. Respir Med. 2015; 109: 1138-46.

17. Vestbo J, Anderson W, Coxson HO, et al. Evaluation of COPD Longitudinally to Identify Predictive Surrogate End-points (ECLIPSE). Eur Respir J. 2008; 31: 869-73.

18. Muller NL, Staples CA, Miller RR and Abboud RT. "Density mask". An objective method to quantitate emphysema using computed tomography. Chest. 1988; 94: 782-7.

19. Park KJ, Bergin CJ and Clausen JL. Quantitation of emphysema with three-dimensional CT densitometry: comparison with two-dimensional analysis, visual emphysema scores, and pulmonary function test results. Radiology. 1999; 211: 541-7.

20. Madani A, Van Muylem A and Gevenois PA. Pulmonary emphysema: effect of lung volume on objective quantification at thin-section CT. Radiology. 2010; 257: 260-8.

21. Rabe KF, Hurd S, Anzueto A, et al. Global strategy for the diagnosis, management, and prevention of chronic obstructive pulmonary disease: GOLD executive summary. Am J Respir Crit Care Med. 2007; 176: 532-55. 
22. Spruit MA, Watkins ML, Edwards LD, et al. Determinants of poor 6-min walking distance in patients with COPD: The ECLIPSE cohort. Respir Med. 2010; 104: 849-57.

23. Spruit MA, Polkey MI, Celli B, et al. Predicting outcomes from 6-minute walk distance in chronic obstructive pulmonary disease. J Am Med Dir Assoc. 2012; 13: 291-7.

24. Polkey MI, Spruit MA, Edwards LD, et al. Six-minute-walk test in chronic obstructive pulmonary disease: minimal clinically important difference for death or hospitalization. Am J Respir Crit Care Med. 2013; 187: 382-6.

25. Fletcher CM. Standardised questionnaire on respiratory symptoms: a statement prepared and approved by the MRC Committee on the Aetiology of Chronic Bronchitis (MRC breathlessness score). Br Med J. 1960; 2: 1665.

26. Jones PW, Quirk FH and Baveystock CM. The St George's Respiratory Questionnaire. Respir Med. 1991; 85: 25-31.

27. Puhan MA, Garcia-Aymerich J, Frey M, et al. Expansion of the prognostic assessment of patients with chronic obstructive pulmonary disease: the updated BODE index and the ADO index. Lancet. 2009; 374: 704-11.

28. Celli BR, Cote CG, Marin JM, et al. The body-mass index, airflow obstruction, dyspnea, and exercise capacity index in chronic obstructive pulmonary disease. N Engl J Med. 2004; 350: 1005-12.

29. Gietema HA, Muller NL, Fauerbach PV, et al. Quantifying the extent of emphysema: factors associated with radiologists' estimations and quantitative indices of emphysema severity using the ECLIPSE cohort. Acad radiol. 2011; 18: 661-71.

30. Bankier AA, De Maertelaer V, Keyzer C and Gevenois PA. Pulmonary emphysema: subjective visual grading versus objective quantification with macroscopic morphometry and thin-section CT densitometry. Radiology. 1999; 211: 851-8.

31. ATS. ATS statement: guidelines for the six-minute walk test. Am J Respir Crit Care Med. 2002; 166: 111-7.

32. DeLong ER, DeLong DM and Clarke-Pearson DL. Comparing the areas under two or more correlated receiver operating characteristic curves: a nonparametric approach. Biometrics. 1988; 44: 837-45.

33. Zafar MA, Tsuang W, Lach L, Eschenbacher W and Panos RJ. Dynamic Hyperinflation Correlates with Exertional Oxygen Desaturation in Patients with Chronic Obstructive Pulmonary Disease. Lung. 2013.

34. Cooper CB. The connection between chronic obstructive pulmonary disease symptoms and hyperinflation and its impact on exercise and function. Am J Med. 2006; 119: 21-31.

35. Loring SH, Garcia-Jacques $M$ and Malhotra A. Pulmonary characteristics in COPD and mechanisms of increased work of breathing. J Appl Physiol. 2009; 107: 309-14.

36. Thurlbeck WM. Internal surface area and other measurements in emphysema. Thorax. 1967; 22: 483-96.

37. Finucane KE and Colebatch HJ. Elastic behavior of the lung in patients with airway obstruction. J App/ Physiol. 1969; 26: 330-8.

38. Kent BD, Mitchell PD and McNicholas WT. Hypoxemia in patients with COPD: cause, effects, and disease progression. Int J Chron Obstruct Pulmon Dis. 2011; 6: 199-208.

39. Mohamed Hoesein FA, de Jong PA, Lammers JW, et al. Contribution of CT quantified emphysema, air trapping and airway wall thickness on pulmonary function in male smokers with and without COPD. COPD. 2014; 11: 503-9.

40. Witting MD and Scharf SM. Diagnostic room-air pulse oximetry: effects of smoking, race, and sex. Am J Emerg Med. 2008; 26: 131-6.

41. Kapur VK, Wilsdon AG, Au D, et al. Obesity is associated with a lower resting oxygen saturation in the ambulatory elderly: results from the cardiovascular health study. Respir Care. 2013; 58: 831-7.

42. Gupta SS, Gothi D, Narula G and Sircar J. Correlation of BMI and oxygen saturation in stable COPD in Northern India. Lung India. 2014; 31: 29-34.

43. Motegi T, Jones RC, Ishii T, et al. A comparison of three multidimensional indices of COPD severity as predictors of future exacerbations. Int J Chron Obstruct Pulmon Dis. 2013; 8: 259-71.

44. Hussein NA, Riet G, Schoenenberger L, et al. The ADO index as a predictor of two-year mortality in general practice-based chronic obstructive pulmonary disease cohorts. Respiration. 2014: 208-14. 


\section{Chapter 5}

45. Cutaia M, Brehm R and Cohen M. The relationship of the BODE index to oxygen saturation during daily activities in patients with chronic obstructive pulmonary disease. Lung. 2011; 189: 269-77.

46. Fiore C, Lee A, McDonald C, Hill C and Holland A. Should oxyhaemoglobin saturation be monitored continuously during the 6-minute walk test? Chron Respir Dis. 2011; 8: 181-4. 


\section{SUPPLEMENTAL MATERIAL}

Table S1. Cut-off points and the ROC curve parameters for the prediction of EID

\begin{tabular}{|c|c|c|c|c|c|c|c|c|}
\hline \multirow[b]{2}{*}{$\begin{array}{l}\text { Predictive } \\
\text { index }\end{array}$} & \multirow[b]{2}{*}{$\begin{array}{l}\text { Best } \\
\text { cut-off }\end{array}$} & \multicolumn{7}{|c|}{ ROC curve parameters } \\
\hline & & $\begin{array}{l}\text { AUC } \\
\text { segment }\end{array}$ & $\begin{array}{l}\text { Sensitivity } \\
(\%)\end{array}$ & $\begin{array}{l}95 \% \mathrm{Cl} \\
\text { Binomial exact }\end{array}$ & $\begin{array}{l}\text { Specificity } \\
(\%)\end{array}$ & $\begin{array}{l}95 \% \mathrm{Cl} \\
\text { Binomial exact }\end{array}$ & $\begin{array}{l}\text { Likelif } \\
\text { Ratio } \\
\text { LR+ }\end{array}$ & $\begin{array}{l}\text { od } \\
\text { LR- }\end{array}$ \\
\hline Age, years & $\geq 60$ & 0.54 & 0.68 & $0.68-0.76$ & 0.35 & $0.32-0.37$ & 1.09 & 0.82 \\
\hline $\mathrm{BMI}, \mathrm{kg} / \mathrm{m}^{2}$ & $\geq 30$ & 0.51 & 0.25 & $0.21-0.29$ & 0.78 & $0.76-0.80$ & 1.16 & 0.96 \\
\hline $\mathrm{FEV}_{1}, \%$ pred. & $\leq 44$ & 0.72 & 0.68 & $0.63-0.72$ & 0.66 & $0.63-0.68$ & 1.96 & 0.49 \\
\hline QCT-Emphysema & $\geq 3$ & 0.71 & 0.72 & $0.55-0.65$ & 0.74 & $0.72-0.77$ & 2.33 & 0.54 \\
\hline $\mathrm{SpO}_{2} \%$ pre-walk & $\leq 93$ & 0.75 & 0.55 & $0.51-0.60$ & 0.81 & $0.79-0.83$ & 2.95 & 0.55 \\
\hline ADO index, score & $\geq 6$ & 0.66 & 0.23 & $0.19-0.27$ & 0.91 & $0.89-0.92$ & 2.42 & 0.85 \\
\hline
\end{tabular}

The best detected threshold values and the ROC parameters for the prediction of EID in the studied COPD population. The area under the curve (AUC) represents the expected performance of the best cut-off points for the predictive indexes. Abbreviations: BMI: Body Mass Index; FEV ${ }_{1} \%$ : Force expiratory volume in $1 \mathrm{sec}$; QCT-emphysema: Quantified by Computed-Tomography emphysema; $\mathrm{SpO}_{2}$ : Saturation of peripheral oxygen; ADO: "Age, Dyspnea, airflow Obstruction" - index.

Table S2. Logistical regressions for ADO index and EID as dependent variable

\begin{tabular}{lllll}
\hline Linear logistic regression & Coefficient & Std. Error & Odds ratio (OR) & P value \\
\hline ADO index & 0.405 & 0.0394 & $1.50(1.39-1.62)$ & $<0.001$ \\
\hline Binary logistic regression & & & & \\
\hline Univariate & Subjects & EID, $n(\%)$ & CRUDE OR & P value \\
\hline ADO index $<6$ points & 1751 & $328(19)$ & 1 & $<0.001$ \\
ADO index $\geq 6$ points & 245 & $97(40)$ & $2.84(2.14-3.78)$ & \\
\hline
\end{tabular}

Linear regression (method: Enter) and binary logistic regression (Univariate) for ADO index and EID as dependent variable. Abbreviation: ADO: “Age, Dyspnea, airflow Obstruction" - index, EID: Exercise-induced desaturation. 


\section{Chapter 5}

Table S3. Pearson's correlations between patients' characteristics, $\mathrm{SpO}_{2} \%$ and $\mathrm{QCT}$-emphysema

\begin{tabular}{|c|c|c|c|c|c|c|}
\hline \multirow[b]{2}{*}{ Characteristics } & \multicolumn{2}{|l|}{$\mathrm{SpO}_{2} \%$ post-walk } & \multicolumn{2}{|c|}{$\Delta \mathrm{SpO}_{2} \%$ [Delta values] } & \multicolumn{2}{|c|}{ QCT-emphysema [degrees] } \\
\hline & $r(95 \% \mathrm{Cl})$ & $P$ value & $r(95 \% \mathrm{Cl})$ & P value & $r(95 \% \mathrm{Cl})$ & P value \\
\hline Age, years & $-0.07(-0.12,0.03)$ & 0.002 & $-0.04(-0.08,0.01)$ & 0.109 & $0.06(0.02,0.11)$ & 0.008 \\
\hline $\mathrm{BMI}, \mathrm{kg} / \mathrm{m}^{2}$ & $-0.04(-0.08,0.01)$ & 0.098 & $0.02(-0.02,0.06)$ & 0.020 & $-0.36(-0.40,-0.32)$ & $<0.001$ \\
\hline FFMI, $\mathrm{kg} / \mathrm{m}^{2}$ & $0.01(-0.03,0.06)$ & 0.517 & $0.07(0.02,0.11)$ & 0.066 & $-0.35(-0.38,-0.30)$ & $<0.001$ \\
\hline FEV1, \% pred. & $0.38(0.34,0.42)$ & $<0.001$ & $0.32(0.28,0.35)$ & $<0.001$ & $-0.41(-0.45,-0.38)$ & $<0.001$ \\
\hline FVC, \% pred. & $0.14(0.10,0.19)$ & $<0.001$ & $0.07(0.03,0.12)$ & 0.001 & $-0.03(-0.01,0.08)$ & 0.173 \\
\hline $\mathrm{SpO}_{2}$ pre, $\%$ & $0.50(0.47,0.53)$ & $<0.001$ & $0.09(0.04,0.13)$ & 0.001 & $-0.06(-0.11,-0.02)$ & 0.005 \\
\hline $\mathrm{SpO}_{2}$ post, $\%$ & 1 & - & $0.89(0.88,0.89)$ & $<0.001$ & $-0.35(-0.39,-0.31)$ & $<0.001$ \\
\hline$\Delta \mathrm{SpO}_{2}, \%$ & $0.89(0.88,0.89)$ & $<0.001$ & & - & $-0.38(-0.41,-0.34)$ & $<0.001$ \\
\hline $\begin{array}{l}\text { QCT- } \\
\text { emphysema }\end{array}$ & $-0.35(-0.39,-0.31)$ & $<0.001$ & $-0.38(-0.41,-0.34)$ & $<0.001$ & $1-$ & - \\
\hline 6MWD, m & $0.17(0.12,0.21)$ & $<0.001$ & $0.08(0.04,0.13)$ & 0.001 & $-0.16(-0.20,-0.11)$ & $<0.001$ \\
\hline 6MWD, \%pred. & $0.15(0.11,0.19)$ & $<0.001$ & $0.08(0.04,0.13)$ & 0.001 & $-0.20(-0.24,0.15)$ & $<0.001$ \\
\hline ADO index & $-0.28(-0.33,-0.24)$ & $<0.001$ & $-0.20(-0.25,-0.16)$ & $<0.001$ & $0.28(0.23,0.32)$ & $<0.001$ \\
\hline BODE index & $-0.30(-0.34,-0.26)$ & $<0.001$ & $-0.22(-0.26,-0.18)$ & $<0.001$ & $0.36(0.31,0.39)$ & $<0.001$ \\
\hline
\end{tabular}

Abbreviations: BMI: Body Mass Index; FFMI: Fat free mass index; FEV ${ }_{1} \%$ : Force expiratory volume in $1 \mathrm{sec}$; FVC: Force vital capacity; $\mathrm{SpO}_{2}$ : Saturation of peripheral oxygen; QCT-emphysema: Quantified by ComputedTomography emphysema; 6MWD: Six-minute walk distance; ADO: "Age, Dyspnea, and airflow Obstruction" index; BODE: "Body mass index, airflow Obstruction, Dyspnea and Exercise capacity" - index.

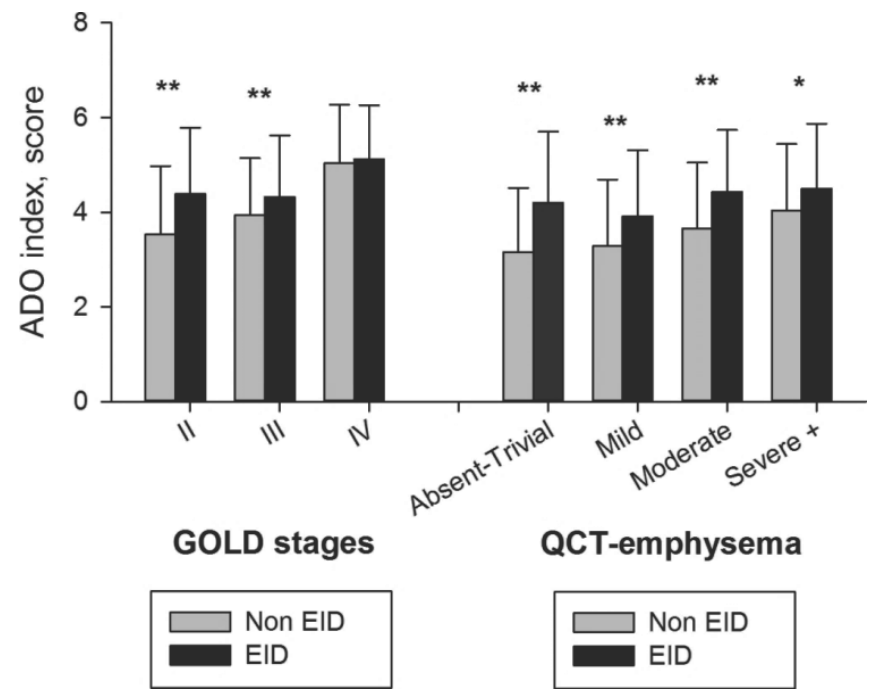

Figure S1. Comparisons of the ADO index between EID and non-EID across the GOLD stages and the degrees of QCT-emphysema

Abbreviations: ADO: "Age, Dyspnea, and airflow Obstruction" - index; EID: Exercise-induced desaturation. Asterisks denote significant difference $(* p<0.05 ; * * p<0.001)$. 


\section{Chapter}

\section{Exercise-induced oxygen desaturation in COPD patients without resting hypoxemia}




\section{Chapter 6}

\section{ABSTRACT}

\section{Background}

Exercise-induced oxygen desaturation (EID) is associated with increased risk of mortality in chronic obstructive pulmonary disease (COPD). Several screening tests have been proposed to predict EID, including $\mathrm{FEV}_{1}, \mathrm{DLCO}$ and baseline- $\mathrm{SpO}_{2}$. We aimed to validate a proposed cut-off of baseline- $\mathrm{SpO}_{2} \leq 95 \%$ as simple screening procedure to predict EID during six-minute walk test (6MWT). In addition, we studied the prevalence and characteristics of patients exhibited EID to $\mathrm{SpO}_{2}$ nadir $\leq 88 \%$.

\section{Methods}

402 non-hypoxemic COPD patients performed 6MWT. Sensitivity and specificity of baseline $\mathrm{SpO}_{2} \leq 95 \%$ as a cut-off to predict EID and determinants of EID were investigated.

\section{Results}

158 patients (39\%) exhibited EID. The sensitivity of baseline-SpO $2 \leq 95 \%$ to predict EID was $81.0 \%$, specificity $49.2 \%$, positive and negative predictive values were $50.8 \%$ and $80.0 \%$, respectively. In a multivariate model, DLCO $<50 \%, \mathrm{FEV}_{1}<45 \%, \mathrm{PaO} 2<10 \mathrm{kPa}$, baseline- $\mathrm{SpO}_{2}<95 \%$, and female sex were the strongest determinants of EID.

\section{Conclusions}

Baseline oxygen saturation solely is inaccurate to predict EID. A combination of clinical characteristics (DLCO, $\mathrm{FEV}_{1}, \mathrm{PaO}_{2}$, baseline-SpO 2 , sex) increases the odds for EID in COPD. 


\section{INTRODUCTION}

Chronic obstructive pulmonary disease (COPD) is a highly prevalent chronic respiratory disease, characterized by partially irreversible airflow limitation. ${ }^{1}$ Patients with COPD frequently experience a significant fall of oxygen saturation during exercise that is attributed to the imbalance between oxygen delivery and demand during exercise. ${ }^{2}$ Exercise-induced oxygen desaturation (EID) has been related to reduced exercise performance, a faster decline in forced expiratory volume in the first second $\left(\mathrm{FEV}_{1}\right)$, and increased mortality in patients with COPD. ${ }^{3,} 4$ Moreover, ambulatory oxygen supplementation reduces exercise-induced breathlessness and oxygen desaturation; and improves exercise performance in patients with COPD, irrespective of resting arterial oxygen pressure. ${ }^{5,6}$ In fact, oxygen saturation $\leq 88 \%$ with physical activity is currently an indication for reimbursement of continuous oxygen use by Centres for Medicare \& Medicaid Services in COPD patients with resting non-hypoxemia. ${ }^{7}$ Therefore, identifying non-hypoxemic COPD patients with EID seems clinically relevant, but has not been studied previously.

A 6-minute walk test (6MWT) has been suggested as preferred measure to identify COPD patients with EID. ${ }^{8}$ Indeed, EID occurs frequently during 6MWT in patients with COPD. ${ }^{9}$ Moreover, the 6MWT is more sensitive for identifying EID in patients with COPD compared to cardiopulmonary exercise test. ${ }^{10}$

Even though the 6MWT is a relatively simple test ${ }^{11}$, several screening tests have been proposed to predict which COPD patients will (not) have EID, including FEV , $_{1}$ diffusion capacity for carbon monoxide (DLCO) and/or baseline standard pulse oxymetry $\left(\mathrm{SpO}_{2}\right)^{8}, 12,13$ Indeed, Knower and colleagues suggested that a resting $\mathrm{SpO}_{2}$ of $95 \%$ or less is a good screening test for the detection of EID during a 6MWT (sensitivity: 73\%; negative predictive value: $84 \%$ ), especially in patients with a DLCO $>36 \%$ of the predicted value (sensitivity: $100 \%$; negative predictive value: $100 \%) .^{8}$ To date, this readily available office screening procedure has not been validated in a large cohort of non-hypoxemic patients with COPD.

Herein, we report a prospective analysis in a large clinical cohort of patients with COPD undergoing baseline evaluation for pulmonary rehabilitation. ${ }^{14}$ The aim of the study was two-fold: 1 ) to validate the cut off of resting $\mathrm{SpO}_{2}$ of $95 \%$ or less as a simple screening procedure to predict EID during a 6MWT in patients with COPD as proposed by Knower and colleagues ${ }^{8}$; and 2) to study the characteristics of non-hypoxemic patients with COPD who have a nadir $\mathrm{SpO}_{2} \leq 88 \%$ during the 6MWT. A priori, resting $\mathrm{SpO}_{2}$ was expected not to be accurate enough to identify non-hypoxemic patients with EID. 


\section{METHODS}

\section{Design and participants}

Patients with COPD ${ }^{15}$ referred for a comprehensive pulmonary rehabilitation program at $\mathrm{CIRO}+$ or its pulmonary rehabilitation network were recruited prospectively. ${ }^{14}$ Age between 45 to 85 years was set as inclusion criterion. The use of long-term oxygen therapy and/or treatment or hospitalisation for an acute exacerbation in the last 4 weeks before the study and resting hypoxemia were pre-defined exclusion criteria. The medical ethical committee of the Maastricht University Medical Centre (METC 11-4042) approved this study and all subjects provided written informed consent for the use of data that were obtained during the routine pre-rehabilitation assessment. Initially, a power calculation was performed, suggesting to include 402 patients (power: $90 \%$; level of significance: 0.01; please see supplemental material for all details). A completed dataset of 402 patients was derived from a total number of 438 eligible patients according the study criteria after the exclusion those who were unable reliably to perform spirometric maneuvers of $\mathrm{FEV}_{1}$, DLCO or the 6MWT.

\section{Assessments}

As part of a 3-day pre-rehabilitation assessment ${ }^{14}$, patients underwent a physical examination by a chest physician (including assessment of body weight, height and medical history), post-bronchodilator spirometry, DLCO, resting arterial blood gas analysis and 6MWTs according to international recommendations. ${ }^{16-19}$ Body mass index (BMI, $\mathrm{kg} / \mathrm{m}^{2}$ ) was calculated as weight divided by squared height. Overweight was defined as a BMI of $25.0-29.9 \mathrm{~kg} / \mathrm{m}^{2}$ and obesity as a BMl of $30.0 \mathrm{~kg} / \mathrm{m}^{2}$ or more. In addition, fat mass (FM) and fat-free mass index (FFMI) (using dual-energy X-ray absorptiometry (DEXA)) ${ }^{20}$ and symptoms of dyspnea (using the Medical Research Council (MRC) dyspnea scale) were assessed.

\section{Six-minute walk test}

Two 6MWTs were performed according to the American Thoracic Society guidelines ${ }^{19}$, including a practice walk. ${ }^{21}$ Participants were asked to walk in a $125 \mathrm{~m}$ triangular track during 6MWTs while $\mathrm{SpO}_{2}$ and heart rate were continuously recorded by the use of pulse oxymeter (Nonin-2500). Both 6MWTs were conducted by healthcare professionals who instructed and supervised the patients while pulse oxymetry during walking tests was monitored for possible artefacts. Patients were encouraged every minute of the 6MWTs using two phrases "You are doing well" or "Keep up the good work". Symptom scores for breathlessness and perceived exertion were recorded using Borg scales. ${ }^{22}$ The 6MWT with the longest walk distance was used for further analyses. 
Acquisition of baseline $\mathrm{SpO}_{2}$ was obtained in sitting position while patients were silent and relaxed. EID was defined as a nadir $\mathrm{SpO}_{2} \leq 88 \%$. A nadir $\mathrm{SpO}_{2} \leq 88 \%$ is current indications for reimbursement for continuous oxygen use by the Centres of Medicare and Medicaid Services. ${ }^{7}$

\section{Statistical analyses}

All statistical analyses were carried out using Sigmaplot 11, GraphPad Prism 4.03 and SPSS 19.0. Data are presented as mean and standard deviation or proportion, as appropriate. For aim 1, patients were stratified by baseline $\mathrm{SpO}_{2} \leq 95 \%$ (yes/no) and the presence of EID (yes/no). Differences between patients with and without EID were compared using an unpaired T-test. In addition, specificity, sensitivity, the positive predictive value and the negative predictive value for baseline $\mathrm{SpO}_{2} \leq 95 \%$ were calculated with standard methods. For aim 2, patients with and without EID were compared using an unpaired T-test. Receiver operating characteristic (ROC) curves were used to determine the threshold values with the best sensitivity and specificity to predict EID, best being defined as the point on the ROC curve with the shortest distance from the upper left corner $(0,1)$. Area under the curve $(A \cup C)$ was calculated by the trapezoidal rule. In addition, univariate and multivariate logistic regression analyses were used to estimate odds ratios for EID. A priori, a two-sided level of significance was set at $p \leq 0.05$.

\section{RESULTS}

\section{Patient characteristics}

A total of 402 patients (age range: 45-85; mean: 64.3 years) with COPD (174 women) were included in this study. Patients generally had moderate to very severe COPD, an impaired lung diffusion capacity, a normal BMI, normal resting arterial oxygen and carbon dioxide tension, severe symptoms of dyspnea at rest, and a poor functional exercise performance. 158 patients (59\%) had EID. Desaturators had a lower BMI, a higher degree of airflow limitation, worse DLCO, more static lung hyperinflation, and worse resting arterial blood gases compared to non-desaturators (Table 1).

\section{Validation of algorithm of Knower et al. (Aim 1)}

252 patients (63\%) had a baseline $\mathrm{SpO}_{2}$ of $95 \%$ or lower, of which 128 patients (51\%) had EID. In the group of patients with a baseline $\mathrm{SpO}_{2}$ of $96 \%$ or higher, 30 patients (20\%) had EID. Desaturators had a higher degree of airflow limitation, worse DLCO, and more static lung hyperinflation compared to non-desaturators, irrespective of the baseline $\mathrm{SpO}_{2}$ 


\section{Chapter 6}

(Table 2). Sensitivity of the baseline $\mathrm{SpO}_{2}$ of $95 \%$ or lower to predict EID was $81.0 \%$; specificity was $49.2 \%$; positive predictive value was $50.8 \%$; and negative predictive value was $80.0 \%$. These numbers hardly changed after excluding all COPD patients with a DLCO of $36 \%$ of predicted or less, as was done by Knower and colleagues ${ }^{8}$ (Table 3 ).

Table 1. Demographics of COPD patients and the prevalence of EID during 6MWT

\begin{tabular}{|c|c|c|c|}
\hline Characteristics & $\begin{array}{l}\text { All Patients } \\
(n=402)\end{array}$ & $\begin{array}{l}\text { Non-Desaturators } \\
(n=244)(61 \%)\end{array}$ & $\begin{array}{l}\text { Desaturators } \\
(n=158)(39 \%)\end{array}$ \\
\hline Women, \#, (\%) & $174(43)$ & $95(39)$ & $79(50)$ \\
\hline Age, years & $64.3 \pm 8.0$ & $64.1 \pm 8.3$ & $64.6 \pm 7.6$ \\
\hline $\mathrm{BMI}, \mathrm{kg} / \mathrm{m}^{2}$ & $25.6 \pm 5.5$ & $26.2 \pm 5.7$ & $24.8 \pm 5.2^{*}$ \\
\hline $\mathrm{FM}, \mathrm{kg}$ & $23.3 \pm 10.5$ & $23.8 \pm 11.0$ & $22.5 \pm 9.8$ \\
\hline FFMI, $\mathrm{kg} / \mathrm{m}^{2}$ & $17.2 \pm 2.4$ & $17.5 \pm 2.3$ & $16.6 \pm 2.4^{*}$ \\
\hline $\mathrm{FEV}_{1}, \mathrm{~L}$ & $1.4 \pm 0.6$ & $1.6 \pm 0.6$ & $1.1 \pm 0.4^{*}$ \\
\hline $\mathrm{FEV}_{1}, \%$ pred. & $51.5 \pm 18.8$ & $58.1 \pm 18.3$ & $41.5 \pm 14.5^{*}$ \\
\hline $\mathrm{FEV}_{1} / \mathrm{FVC}, \%$ & $40.2 \pm 12.1$ & $44.5 \pm 11.7$ & $33.6 \pm 9.5^{*}$ \\
\hline FVC, \%pred. & $99.3 \pm 21.6$ & $101.2 \pm 21.5$ & $96.3 \pm 21.5^{*}$ \\
\hline FRC, \%pred. & $143.8 \pm 34.1$ & $134.7 \pm 31.6$ & $157.9 \pm 33.0^{*}$ \\
\hline TLC, \%pred. & $115.8 \pm 17.1$ & $112.8 \pm 16.4$ & $120.4 \pm 17.2^{*}$ \\
\hline DLCO, \%pred. & $53.9 \pm 19.7$ & $60.4 \pm 19.9$ & $43.7 \pm 14.4^{*}$ \\
\hline $\mathrm{PaO}_{2}, \mathrm{kPa}$ & $10.1 \pm 1.6$ & $10.5 \pm 1.5$ & $9.4 \pm 1.4^{*}$ \\
\hline $\mathrm{PaCO}_{2}, \mathrm{kPa}$ & $5.0 \pm 0.6$ & $4.9 \pm 0.5$ & $5.2 \pm 0.7^{*}$ \\
\hline $\mathrm{SaO}_{2}, \%$ & $95.5 \pm 2.0$ & $96.1 \pm 1.6$ & $94.7 \pm 2.2$ \\
\hline $\mathrm{SpO}_{2}, \%$ & $94.6 \pm 2.4$ & $95.4 \pm 1.9$ & $93.3 \pm 2.5^{*}$ \\
\hline 6MWD, m & $453.5 \pm 103.2$ & $458.8 \pm 104.9$ & $445.3 \pm 100.1$ \\
\hline 6MWD, \%pred. & $70.8 \pm 16.4$ & $71.8 \pm 15.7$ & $69.3 \pm 17.2$ \\
\hline$M R C$, index & $3.2 \pm 0.9$ & $3.2 \pm 0.9$ & $3.3 \pm 0.9$ \\
\hline
\end{tabular}

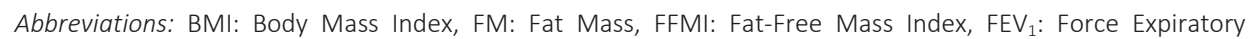
Volume at $1 \mathrm{sec}$, FVC: Force Vital Capacity, FRC: Functional Residual Capacity, TLC: Total Lung Capacity, DLCO: Diffusing capacity for carbon monoxide, $\mathrm{PO}_{2}$ : Partial pressure of Oxygen, $\mathrm{PCO}_{2}$ : Partial pressure of Carbondioxide, $\mathrm{SaO}_{2}$ : Arterial oxygen saturation, $\mathrm{SpO}_{2}$ : Saturation of peripheral Oxygen, 6MWD: Six-minute walk distance, MRC: Medical Research Council dyspnea scale. Data is represented as means \pm SD and percentages (\%). Data are mean $\pm S D$ unless specified otherwise. Asterisk denotes significant differences for patients exhibited EID (Desaturators) $(P \leq 0.05)$. 
Table 2. Characteristics of COPD patients stratified by baseline saturation and grouped based on the presence of EID during 6MWT

\begin{tabular}{|c|c|c|c|c|}
\hline \multirow[b]{2}{*}{ Characteristics } & \multicolumn{2}{|c|}{ Baseline $\mathrm{SpO}_{2} \geq 96 \%(\mathrm{n}=150)$} & \multicolumn{2}{|c|}{ Baseline $\mathrm{SpO}_{2} \leq 95 \%(\mathrm{n}=252)$} \\
\hline & $\begin{array}{l}\text { Non-Desaturators } \\
(n=120)(80 \%)\end{array}$ & $\begin{array}{l}\text { Desaturators } \\
(n=30)(20 \%)\end{array}$ & $\begin{array}{l}\text { Non-Desaturators } \\
(n=124)(49 \%)\end{array}$ & $\begin{array}{l}\text { Desaturators } \\
(n=128)(51 \%)\end{array}$ \\
\hline Women, \#, (\%) & $50(42)$ & $22(73)$ & $45(36)$ & $57(45)$ \\
\hline Age, years & $62.8 \pm 8.6$ & $61.8 \pm 8.1$ & $65.3 \pm 7.8$ & $65.3 \pm 7.4$ \\
\hline $\mathrm{BMI}, \mathrm{kg} / \mathrm{m}^{2}$ & $25.4 \pm 5.5$ & $23.0 \pm 4.3^{*}$ & $27.0 \pm 5.7$ & $25.2 \pm 5.4^{*}$ \\
\hline $\mathrm{FM}, \mathrm{kg}$ & $21.9 \pm 10.1$ & $20.6 \pm 8.9$ & $25.5 \pm 11.5$ & $22.9 \pm 9.9$ \\
\hline $\mathrm{FFMI}, \mathrm{kg} / \mathrm{m}^{2}$ & $17.3 \pm 2.3$ & $15.7 \pm 2.2^{*}$ & $17.8 \pm 2.3$ & $16.8 \pm 2.4^{*}$ \\
\hline $\mathrm{FEV}_{1}, \mathrm{~L}$ & $1.7 \pm 0.6$ & $1.1 \pm 0.4$ & $1.5 \pm 0.6$ & $1.1 \pm 0.4^{*}$ \\
\hline $\mathrm{FEV}_{1}, \%$ pred. & $60.1 \pm 18.9$ & $41.9 \pm 14.6^{*}$ & $56.1 \pm 17.6$ & $41.4 \pm 14.6^{*}$ \\
\hline $\mathrm{FEV}_{1} / \mathrm{FVC}, \%$ & $45.5 \pm 11.8$ & $34.6 \pm 9.9 *$ & $43.5 \pm 11.6$ & $33.4 \pm 9.4 *$ \\
\hline FVC, \%pred. & $102.9 \pm 20.7$ & $95.2 \pm 18.3$ & $99.7 \pm 22.3$ & $96.6 \pm 22.2$ \\
\hline FRC, \%pred. & $133.4 \pm 31.0$ & $163.1 \pm 27.9^{*}$ & $135.8 \pm 32.3$ & $156.7 \pm 34.1^{*}$ \\
\hline TLC, \%pred. & $113.1 \pm 16.1$ & $125.0 \pm 15.9 *$ & $112.6 \pm 16.8$ & $119.3 \pm 17.4^{*}$ \\
\hline DLCO, \%pred. & $60.8 \pm 22.2$ & $42.2 \pm 15.5^{*}$ & $60.2 \pm 17.5$ & $44.0 \pm 14.2 *$ \\
\hline $\mathrm{PaO}_{2}, \mathrm{kPa}$ & $10.9 \pm 1.4$ & $10.1 \pm 1.4^{*}$ & $10.1 \pm 1.6$ & $9.2 \pm 1.4^{*}$ \\
\hline $\mathrm{PaCO}_{2}, \mathrm{kPa}$ & $4.8 \pm 0.5$ & $5.0 \pm 0.6$ & $5.0 \pm 0.5$ & $5.2 \pm 0.7^{*}$ \\
\hline $\mathrm{SpO}_{2}, \%$ & $97.0 \pm 0.8$ & $96.4 \pm 0.7^{*}$ & $93.8 \pm 1.3$ & $92.6 \pm 2.1^{*}$ \\
\hline 6MWD, m & $468.5 \pm 110.3$ & $488.9 \pm 84.5$ & $449.4 \pm 99.0$ & $435.1 \pm 101.1$ \\
\hline 6MWD, \%pred. & $72.6 \pm 16.9$ & $76.1 \pm 13.1$ & $71.0 \pm 14.5$ & $67.6 \pm 17.7$ \\
\hline MRC, index & $3.1 \pm 1.0$ & $2.9 \pm 0.9$ & $3.2 \pm 0.9$ & $3.4 \pm 0.9$ \\
\hline
\end{tabular}

Abbreviations: Please see legend Table 1. The studied COPD patients categorized by baseline saturation of $\mathrm{SpO}_{2} \geq 96 \%$ and $\mathrm{SpO}_{2} \leq 95 \%$ and grouped based on the presence of EID to nadir- $\mathrm{SpO}_{2} \leq 88 \%$. Data are mean \pm SD unless specified otherwise. Asterisk denotes significant differences for patients exhibited EID (Desaturators) $(P \leq 0.05)$.

Table 3. Sensitivity and specificity of the parameters tested as predictors of EID in COPD

\begin{tabular}{lllll}
\hline Predictive index & $\begin{array}{l}\text { Sensitivity } \\
(\%)\end{array}$ & $\begin{array}{l}\text { Specificity } \\
(\%)\end{array}$ & $\begin{array}{l}\text { Positive predictive } \\
\text { value (\%) }\end{array}$ & $\begin{array}{l}\text { Negative predictive } \\
\text { value (\%) }\end{array}$ \\
\hline $\begin{array}{l}\text { Baseline Saturation 95\% } \\
\text { (Total Sample) }\end{array}$ & 81.0 & 49.2 & 50.8 & 80.0 \\
$\begin{array}{l}\text { Baseline Saturation 95\% } \\
\text { (Excluding DLCO } \leq 36 \%)\end{array}$ & 84.5 & 48.0 & 42.4 & 87.2 \\
\hline
\end{tabular}

A cut-off of baseline saturation of $95 \%$ presented low sensitivity to predict EID to nadir-SpO $2 \leq 88 \%$ in the total sample of patients with COPD and after the exclusion those with DLCO $\leq 36 \%$.

\section{Exercise-induced nadir $\mathrm{SpO}_{2} \leq 88 \%$ (Aim 2)}

Using ROC curves, the threshold values with the best specificity and sensitivity to predict EID were determined for age, $\mathrm{BMI}, \mathrm{FEV}{ }_{1}, \mathrm{FRC}, \mathrm{DLCO}, \mathrm{PaO}_{2}, \mathrm{PaCO}_{2}$, and baseline $\mathrm{SpO}_{2}$ (Table 4). These threshold values were used in an univariate logistical regression model, which revealed that patients aged 60 years or older; with an overweight or 


\section{Chapter 6}

obese BMI; with $\mathrm{FEV}_{1}<45 \%$ predicted; with $\mathrm{FRC}>135$ \%predicted; with a DLCO $<50$ \%predicted; or with a $\mathrm{PaO}_{2}<10.0 \mathrm{kPa}$; $\mathrm{PaCO}_{2} \geq 5.2 \mathrm{kPa}$ and baseline $\mathrm{SpO}_{2}$ of $95 \%$ or lower have higher odds for EID (Table 5). A multivariate logistical regression model, however, showed that only $\mathrm{FEV}_{1}, \mathrm{DLCO}, \mathrm{PaO}_{2}$ and $\mathrm{SpO}_{2}$ remained significant. A combination of these critical characteristics revealed that the $95 \%$ of patients with $\mathrm{FEV}_{1}<45$ \%predicted and DLCO $<50$ \%predicted with resting $\mathrm{PaO}_{2}<10 \mathrm{kPa}$ and baseline $\mathrm{SpO}_{2}<95 \%$ predicted, exhibited EID (Figure 1). Moreover, in the multivariate logistical regression model the gender of patients was revealed as an important factor for the prevalence of EID indicating a higher proportion of women who exhibited EID (Table 5 and Figure 1).

Table 4. Cut-off points and the ROC curve parameters for the prediction of EID

\begin{tabular}{|c|c|c|c|c|c|c|}
\hline \multicolumn{7}{|c|}{ ROC curve parameters } \\
\hline \multirow{2}{*}{$\begin{array}{l}\text { Predictive } \\
\text { index }\end{array}$} & \multirow{2}{*}{$\begin{array}{l}\text { Best } \\
\text { cut-off }\end{array}$} & \multirow{2}{*}{$\begin{array}{l}\text { AUC } \\
\text { segment }\end{array}$} & \multirow[b]{2}{*}{ Sensitivity (\%) } & \multirow[b]{2}{*}{ Specificity (\%) } & \multicolumn{2}{|c|}{ Likelihood Ratio } \\
\hline & & & & & $\mathrm{LR}+$ & LR- \\
\hline Age, years & 59.5 & 0.52 & 0.76 & 0.35 & 1.17 & 0.69 \\
\hline $\mathrm{BMI}, \mathrm{kg} / \mathrm{m}^{2}$ & 25.8 & 0.58 & 0.68 & 0.50 & 1.37 & 0.63 \\
\hline Baseline $\mathrm{SpO}_{2}, \%$ & 94.5 & 0.74 & 0.65 & 0.70 & 2.13 & 0.51 \\
\hline $\mathrm{PaO}_{2}, \mathrm{kPa}$ & 9.95 & 0.74 & 0.73 & 0.65 & 2.11 & 0.41 \\
\hline $\mathrm{PaCO}_{2}, \mathrm{kPa}$ & 5.25 & 0.61 & 0.42 & 0.78 & 1.92 & 0.74 \\
\hline DLCO, \%pred. & 50.5 & 0.78 & 0.75 & 0.67 & 2.30 & 0.37 \\
\hline $\mathrm{FEV}_{1}, \%$ pred. & 44.5 & 0.76 & 0.67 & 0.76 & 2.77 & 0.43 \\
\hline FRC, \%pred. & 135.5 & 0.69 & 0.75 & 0.54 & 1.63 & 0.46 \\
\hline
\end{tabular}

Abbreviations: $\mathrm{BMI}$ : Body Mass Index, $\mathrm{SpO}_{2}$ : Saturation of peripheral Oxygen, $\mathrm{PO}_{2}$ : Partial pressure of Oxygen, $\mathrm{PCO}_{2}$ : Partial pressure of Carbon-dioxide, DLCO: Diffusing capacity for carbon monoxide, FEV $\mathrm{f}_{1}$ : Force Expiratory Volume at $1 \mathrm{sec}$, FRC: Functional Residual Capacity. The best detected cut-off points and the ROC parameters for the prediction of EID in the studied COPD population. The area under the curve (AUC) represents the expected performance of the best cut-off points for the predictive indexes. 
Exercise-induced oxygen desaturation in COPD patients without resting hypoxemia

Table 5. Logistic regression: determinants of EID in COPD patients

\begin{tabular}{|c|c|c|c|c|c|c|}
\hline Determinants & $\begin{array}{l}\text { Subjects } \\
\mathrm{n}\end{array}$ & $\begin{array}{l}\text { EID } \\
n,(\%)\end{array}$ & Crude OR $(95 \% \mathrm{Cl})$ & $\begin{array}{l}P \\
\text { value }\end{array}$ & $\begin{array}{l}\text { Adjust OR } \\
(95 \% \mathrm{Cl})\end{array}$ & $\begin{array}{l}P \\
\text { value }\end{array}$ \\
\hline \multicolumn{7}{|l|}{ Age } \\
\hline$<60$ years & 123 & $38(31)$ & 1 & & 1 & \\
\hline$\geq 60$ years & 279 & $120(43)$ & $1.69(1.08-2.65)$ & 0.02 & $1.67(0.91-3.06)$ & 0.10 \\
\hline \multicolumn{7}{|l|}{ Sex } \\
\hline Male & 228 & $79(35)$ & 1 & & 1 & \\
\hline Female & 174 & $70(45)$ & $1.57(1.05-2.35)$ & 0.03 & $2.26(1.30-3.92)$ & 0.004 \\
\hline \multicolumn{7}{|l|}{ BMI } \\
\hline$<25 \mathrm{~kg} / \mathrm{m}^{2}$ & 205 & $95(46)$ & 1 & & 1 & \\
\hline$\geq 25 \mathrm{~kg} / \mathrm{m}^{2}$ & 197 & $63(32)$ & $0.54(0.36-0.82)$ & 0.003 & $1.13(0.62-2.09)$ & 0.69 \\
\hline \multicolumn{7}{|l|}{$\mathrm{FEV}_{1}$} \\
\hline$\geq 45 \%$ pred. & 237 & $52(22)$ & 1 & & 1 & \\
\hline$<45$ \%pred. & 165 & $106(64)$ & 6.39 (4.11-9.95) & $<0.001$ & $4.92(2.58-9.41)$ & $<0.001$ \\
\hline \multicolumn{7}{|l|}{ FRC } \\
\hline$\leq 135 \%$ pred. & 170 & $39(23)$ & 1 & & 1 & \\
\hline >135 \%pred. & 232 & $119(51)$ & $3.54(2.28-5.50)$ & $<0.001$ & $1.05(0.54-2.07)$ & 0.88 \\
\hline \multicolumn{7}{|l|}{ DLCO } \\
\hline$\geq 50$ \%pred. & 211 & $43(20)$ & 1 & & 1 & \\
\hline$<50$ \%pred. & 191 & $115(60)$ & $5.91(3.80-9.21)$ & $<0.001$ & $5.45(2.96-10.01)$ & $<0.001$ \\
\hline \multicolumn{7}{|l|}{$\mathrm{PaO}_{2}$} \\
\hline$\geq 10.0 \mathrm{kPa}$ & 201 & $42(21)$ & 1 & & 1 & \\
\hline$<10.0 \mathrm{kPa}$ & 201 & $116(58)$ & $5.17(3.33-8.03)$ & $<0.001$ & $4.89(2.75-8.69)$ & $<0.001$ \\
\hline \multicolumn{7}{|l|}{$\mathrm{PaCO}_{2}$} \\
\hline$<5.2 \mathrm{kPa}$ & 251 & $82(33)$ & 1 & & 1 & \\
\hline$\geq 5.2 \mathrm{kPa}$ & 151 & $76(50)$ & $2.09(1.38-3.16)$ & $<0.001$ & $1.49(0.84-2.63)$ & 0.17 \\
\hline \multicolumn{7}{|l|}{$\mathrm{SpO}_{2}$} \\
\hline$\geq 96 \%$ & 150 & $30(20)$ & 1 & & 1 & \\
\hline$<95 \%$ & 252 & $128(51)$ & $4.13(2.58-6.61)$ & $<0.001$ & $2.25(1.20-4.23)$ & 0.01 \\
\hline
\end{tabular}

Abbreviations: Please see legend of Table 4. Logistic regression analysis of the determinants for EID: adjusted odds ratios for EID according to gender and physiological characteristics.

\section{A posteriori analyses}

Characteristics of desaturators and non-desaturators were studied after stratification for gender (Supplementary table S1). This posteriori analysis shows again that desaturators had a lower BMI, a higher degree of airflow limitation, worse DLCO, more static lung hyperinflation, and worse resting arterial blood gases compared to nondesaturators, irrespective of sex. 
Chapter 6
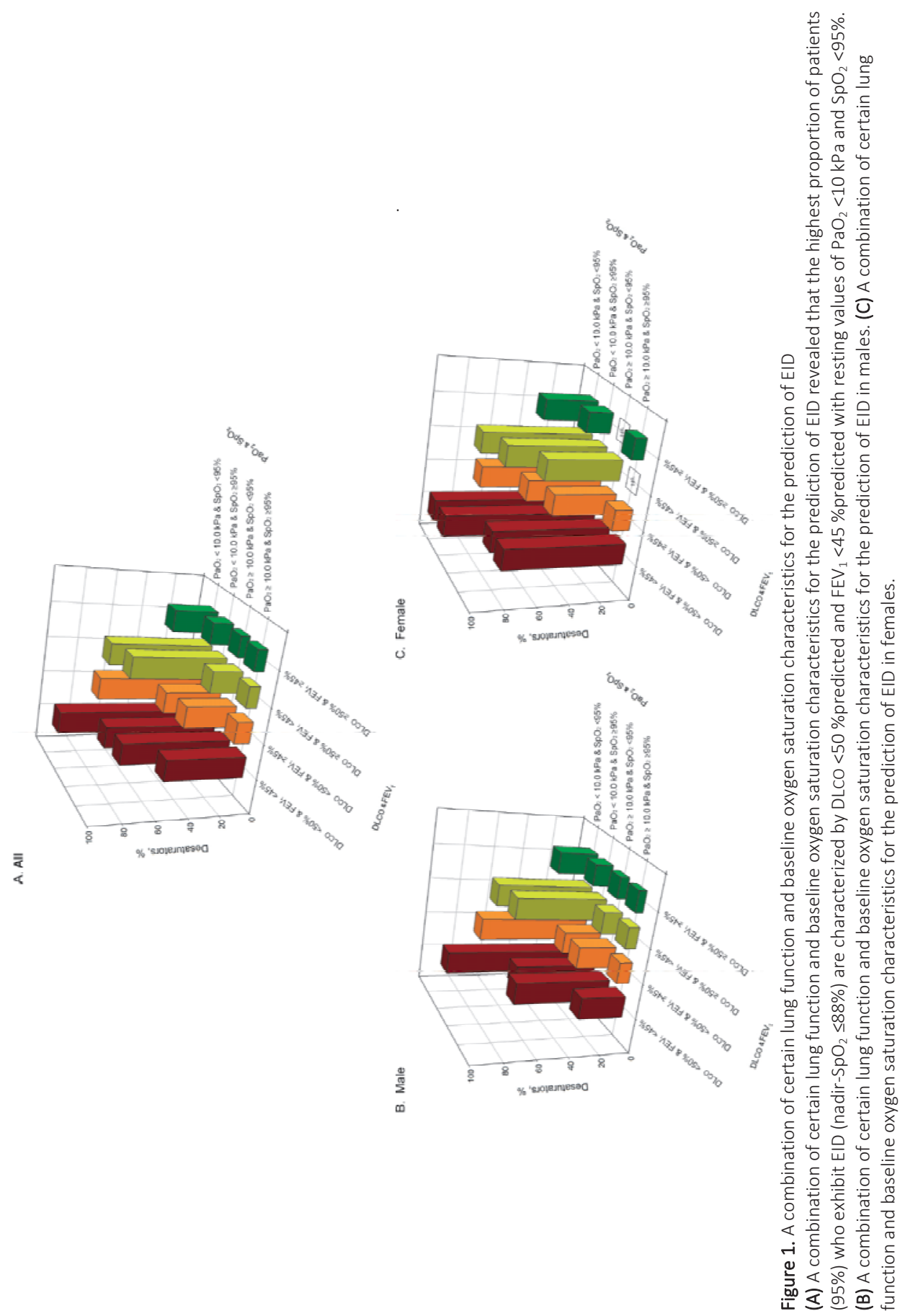


\section{DISCUSSION}

The current study has two main findings: 1) Baseline oxygen saturation threshold of $95 \%$ or lower measured by standard pulse oxymetry fails to identify accurately EID in 402 non-hypoxemic patients with COPD; and 2) Nadir $\mathrm{SpO}_{2} \leq 88 \%$ occurs frequently during a 6MWT in non-hypoxemic patients with COPD, in particular in those patients with a $\mathrm{FEV}_{1}<45 \%$ predicted, DLCO $<50$ \%predicted, resting $\mathrm{PaO}_{2}<10 \mathrm{kPa}$, baseline $\mathrm{SpO}_{2}$ $<95 \%$, and in women.

In 2001, Knower and colleagues reported that baseline oxygen saturation of 95\% or lower is a valid and readily available office screening procedure to identify COPD patients without EID during 6MWT, especially in patients with DLCO greater than 36 \%predicted. ${ }^{8}$ The analyses using a dataset of 402 non-hypoxemic patients with COPD did not corroborate the study of Knower and colleagues. Based on the power calculation (see supplemental materials for all details), a type II error can be ruled out, and, in turn, it seems fair to state that a baseline pulse oximetry threshold of $95 \%$ or lower cannot be used solely in daily clinical practice to identify accurately patients with COPD without EID.

Patients with COPD exhibit EID due to the imbalance between oxygen supply and demand during exercise, which can be attributed to respiratory, hemodynamic and peripheral muscle abnormalities or a combination thereof. ${ }^{23}$ Impaired lung mechanical characteristics and gas exchange inefficiency are prominent in COPD and contribute to increased work of breathing. Impaired hemodynamics, lower-limb muscle dysfunction and weakness ${ }^{24,25}$ in the presence of premature lactic acidosis may negatively impact the dynamic matching of oxygen delivery and utilization. ${ }^{26}$ Arterial hypoxemia and excessive respiratory muscle work are related to insufficient oxygen transport in the working muscles enhancing EID. ${ }^{27}$

In the present study, the six-minute walk test (6MWT) due to the simplicity of performance and the high responsiveness to arterial oxygen desaturation is the preferred exercise test to investigate the EID. It has been shown that 6MWT is more sensitive to arterial oxygen desaturation than the cardiopulmonary exercise test on cycle ergometer. For example, Poulain and colleagues showed that $\sim 28 \%$ of COPD patients with broad range of $\mathrm{FEV}_{1}(29-81 \%$ predicted) desaturate on 6MWT while this was not shown on cardiopulmonary exercise test. ${ }^{10}$ However, authors of this study and following relevant studies did not provide an explanation of this phenomenon ${ }^{2,28,29}$, nor did direct arterial blood gas measurements. ${ }^{30}$ On the other hand, Cockcroft and colleagues showed that a significantly lower $\mathrm{PaO}_{2}$ was present with matching metabolic rates on self-paced walking exercise on treadmill and on the cycle ergometer, and that this difference was accountable for a lower lactate threshold in cycling exercise. ${ }^{31}$ Later, Hsia and colleagues confirmed that the higher minute ventilation found during cycle exercise at the same equivalent work rate (and oxygen uptake) was in close association 
with less EID. ${ }^{32}$ These observations provided a reasonable pathophysiological mechanism for EID.

In the current study, we also focussed on spirometry, lung diffusion capacity, pulse oximetry, and arterial blood gases, which may be available for many patients with COPD from outpatient routine follow ups. As expected, a lower $\mathrm{FEV}_{1}, \mathrm{DLCO}, \mathrm{PaO}_{2}$ and baseline $\mathrm{SpO}_{2}$ increased the odds of having EID (Table 4, and Figure 1). Previously, FEV 1 and DLCO have already been suggested as possible strong predictors of EID in patients with COPD. ${ }^{8,12,13}$ For example, Owens and colleagues ${ }^{13}$ reported that DLCO of 55\% or higher was $100 \%$ specific in identifying patients with COPD without EID, as compared with an $82 \%$ specificity for an $\mathrm{FEV}_{1}$ above $55 \%$ of predicted. The sensitivity of the diffusing capacity with this cutoff point was $68 \%$, as compared with $46 \%$ for the FEV 1 . Kelly and colleagues $^{12}$ showed that patients with COPD or interstitial lung disease with DLCO of $60 \%$ of predicted or higher never experienced EID.

Recently, Van Gestel and colleagues ${ }^{33}$ found that a FEV of $^{5} 0$ \%predicted or lower was the optimal cut-off value to identify COPD patients with EID. Moreover, in a recent study of Crisafulli and colleagues ${ }^{34}$ a multivariate regression between anthropometric and functional variables and $\mathrm{SpO}_{2}$ nadir as the dependent variable indicated that resting saturation of arterial oxygen - $\mathrm{SO}_{2}(r=0.65), \mathrm{PaO}_{2}(r=0.50)$ and $\mathrm{FEV}_{1} \%$ predicted $(r=$ 0.41) significantly predict EID in COPD. Indeed, a walking desaturation score (WDS) derived from these clinical variables classified and accurately predicted the risk of EID in patients with COPD. In accordance with these findings, the present study indicated $\mathrm{FEV}_{1}$ $<45$ \%predicted in association with resting $\mathrm{SpO}_{2}<95 \%$ and $\mathrm{PaO}_{2}<10.0 \mathrm{kPa}$ as determinants of EID. However, the application of WDS in the current study sample classified $77.5 \%$ of the non-desaturating patients as "low risk", 13.9\% as "high risk" and $8.6 \%$ as "very high risk" for EID. Thirty-eight percent of the patients with EID were classified as "low risk", 27.2\% as "high risk", and 34.8\% as "very high risk" for EID. In present study the influence of a low DLCO $<50 \%$ predicted in the prevalence of EID was crucial and the fact that DLCO is not included in the WDS variables may be possibly attributed for the small percentage of high risk group classification of the desaturators of this study.

Interestingly, EID appears also to be influenced by gender. The multivariate logistic regression model of this study revealed that women have higher odds to exhibit EID compared to men (Table 5 and Figure 1). The current analyses cannot explain these findings, which seems rather hypothesis-generating than definitive.

There is no uniform definition of EID in patients with COPD or standardized exercise protocol to elicit decreases in oxygen levels in individuals with COPD. ${ }^{8,13,29,33}$ Nadir $\mathrm{SpO}_{2} \leq 88 \%$ was used in the current study, as the clinical relevance of a decrease of $4 \%$ or more, highly depends on the baseline $\mathrm{SpO}_{2}$. A 6MWT was used to induce oxygen desaturation, as this has been shown to be able to identify EID in patients with COPD. ${ }^{10}$ Oxygen saturation was monitored continuously during the 6MWT for the detection of 
nadir $\mathrm{SpO}_{2} \leq 88 \%$, as $\mathrm{SpO}_{2}$ at the end of the 6MWT did not accurately identify desaturation in $21 \%$ of patients with COPD. ${ }^{35}$

\section{CONCLUSIONS}

In summary, EID cannot be predicted accurately by just using resting $\mathrm{SpO}_{2}$. Moreover, the current analysis revealed that $39 \%$ of the 402 non-hypoxemic patients with COPD had nadir $\mathrm{SpO}_{2} \leq 88 \%$, which may be suitable candidates for ambulatory oxygen therapy. Obviously, future trials have to corroborate the current findings, in particular the observed influence of gender. 


\section{REFERENCES}

1. Vestbo J, Hurd SS, Agusti AG, et al. Global strategy for the diagnosis, management, and prevention of Chronic Obstructive Pulmonary Disease: GOLD executive summary. Am J Respir Crit Care Med. 2013; 187: 347-65.

2. Vogiatzis I and Zakynthinos S. Factors limiting exercise tolerance in Chronic Lung Diseases. Compr Physiol. 2012; 2: 1779-817.

3. Casanova C, Cote C, Marin JM, et al. Distance and oxygen desaturation during the 6-min walk test as predictors of long-term mortality in patients with COPD. Chest. 2008; 134: 746-52.

4. Kim C, Seo JB, Lee SM, et al. Exertional desaturation as a predictor of rapid lung function decline in COPD. Respiration 2012; 86: 109-16.

5. Jolly EC, Di Boscio V, Aguirre L, Luna CM, Berensztein S and Gene RJ. Effects of supplemental oxygen during activity in patients with advanced COPD without severe resting hypoxemia. Chest. 2001; 120: 43743.

6. Somfay A, Porszasz J, Lee SM and Casaburi R. Dose-response effect of oxygen on hyperinflation and exercise endurance in nonhypoxaemic COPD patients. Eur Respir J. 2001; 18: 77-84.

7. Stoller JK, Panos RJ, Krachman S, Doherty DE and Make B. Oxygen therapy for patients with COPD: current evidence and the long-term oxygen treatment trial. Chest. 2010; 138: 179-87.

8. Knower MT, Dunagan DP, Adair NE and Chin R, Jr. Baseline oxygen saturation predicts exercise desaturation below prescription threshold in patients with chronic obstructive pulmonary disease. Arch Intern Med. 2001; 161: 732-6.

9. Jenkins S and Cecins N. Six-minute walk test: observed adverse events and oxygen desaturation in a large cohort of patients with chronic lung disease. Intern Med J. 2011; 41: 416-22.

10. Poulain M, Durand F, Palomba B, et al. 6-minute walk testing is more sensitive than maximal incremental cycle testing for detecting oxygen desaturation in patients with COPD. Chest. 2003; 123: 1401-7.

11. Salzman SH. The 6-min walk test: clinical and research role, technique, coding, and reimbursement. Chest 2009; 135: 1345-52.

12. Kelley MA, Panettieri RA, Jr. and Krupinski AV. Resting single-breath diffusing capacity as a screening test for exercise-induced hypoxemia. Am J Med. 1986; 80: 807-12.

13. Owens GR, Rogers RM, Pennock BE and Levin D. The diffusing capacity as a predictor of arterial oxygen desaturation during exercise in patients with chronic obstructive pulmonary disease. N Engl J Med. 1984; 310: 1218-21.

14. Spruit MA, Vanderhoven-Augustin I, Janssen PP and Wouters EF. Integration of pulmonary rehabilitation in COPD. Lancet. 2008; 371: 12-3.

15. Decramer M, Bartsch P, Pauwels R and Yernault JC. Management of COPD according to guidelines. A national survey among Belgian physicians. Monaldi Arch Chest Dis. 2003; 59: 62-80.

16. Macintyre N, Crapo RO, Viegi G, et al. Standardisation of the single-breath determination of carbon monoxide uptake in the lung. Eur Respir J. 2005; 26: 720-35.

17. Miller MR, Hankinson J, Brusasco V, et al. Standardisation of spirometry. Eur Respir J. 2005; 26: 319-38.

18. Spruit MA, Pennings HJ, Janssen PP, et al. Extra-pulmonary features in COPD patients entering rehabilitation after stratification for MRC dyspnea grade. Respir Med. 2007; 101: 2454-63.

19. ATS. ATS statement: guidelines for the six-minute walk test. Am J Respir Crit Care Med. 2002; 166: 111-7.

20. Graat-Verboom L, van den Borne BE, Smeenk FW, Spruit MA and Wouters EF. Osteoporosis in COPD outpatients based on bone mineral density and vertebral fractures. Bone Miner. Res. Off. J. Am. Soc. Bone Miner. Res. 2011; 26: 561-8.

21. Hernandes NA, Wouters EF, Meijer K, Annegarn J, Pitta F and Spruit MA. Reproducibility of 6-minute walking test in patients with COPD. Eur Respir J. 2011; 38: 261-7.

22. Rozkovec A, Papouchado M, James MA, Kendrick AH, Clarke LM and Rees JR. The relationship of symptoms to performance in paced patients with breathlessness. Eur Heart J. 1989; 10: 63-9. 
23. Vogiatzis I, Zakynthinos $G$ and Andrianopoulos V. Mechanisms of physical activity limitation in Chronic Lung Diseases. Pulm Med. 2012; (2012): 1-11.

24. Decramer M, De Benedetto F, Del Ponte A and Marinari S. Systemic effects of COPD. Respir Med. 2005; 99: S3-10.

25. Franssen FM, Broekhuizen R, Janssen PP, Wouters EF and Schols AM. Limb muscle dysfunction in COPD: effects of muscle wasting and exercise training. Med Sci Sports Exerc. 2005; 37: 2-9.

26. Maltais F, Jobin J, Sullivan MJ, et al. Metabolic and hemodynamic responses of lower limb during exercise in patients with COPD. J Appl Physiol. 1998; 84: 1573-80.

27. Amann M, Regan MS, Kobitary M, et al. Impact of pulmonary system limitations on locomotor muscle fatigue in patients with COPD. Am J Physiol Regul Integr Comp Physiol. 2010; 299: R314-24.

28. Palange $\mathrm{P}, \mathrm{W}$ ard $\mathrm{S}$, Carlsen $\mathrm{K}$, et al. Recommendation on the use of exercise testing in clinical practice. Eur Respir J. 2007; 29: 185-209.

29. Panos RJ and Eschenbacher W. Exertional desaturation in patients with chronic obstructive pulmonary disease. COPD. 2009; 6: 478-87.

30. Mahler DA, Gifford AH, Waterman LA, Ward J, Machala S and Baird JC. Mechanism of greater oxygen desaturation during walking compared with cycling in patients with COPD. Chest. 2011; 140: 351-8.

31. Cockcroft A, Beaumont A, Adams $L$ and Guz A. Arterial oxygen desaturation during treadmill and bicycle exercise in patients with chronic obstructive airways disease. Clin Sci. 1985; 68: 327-32.

32. Hsia D, Casaburi R, Pradhan A, Torres E and Porszasz J. Physiological responses to linear treadmill and cycle ergometer exercise in COPD. Eur Respir J. 2009; 34: 605-15.

33. van Gestel AJ, Clarenbach CF, Stowhas AC, et al. Prevalence and prediction of exercise-induced oxygen desaturation in patients with chronic obstructive pulmonary disease. Respiration. 2012; 84: 353-9.

34. Crisafulli E, lattoni A, Venturelli E, et al. Predicting Walking-induced oxygen desaturations in COPD patients: A statistical model. Respir Care. 2013; 58: 1495-503.

35. Fiore C, Lee A, McDonald C, Hill C and Holland A. Should oxyhaemoglobin saturation be monitored continuously during the 6-minute walk test? Chron Respir Dis. 2011; 8: 181-4. 
Chapter 6

\section{SUPPLEMENTAL MATERIAL}

Table S1. Characteristics of COPD patients stratified by gender and grouped based on the presence of EID

\begin{tabular}{|c|c|c|c|c|c|c|c|c|}
\hline \multirow{3}{*}{$\frac{\text { Characteristics }}{\text { Age, years }}$} & \multicolumn{4}{|c|}{ MALES $(n=228)$} & \multicolumn{4}{|c|}{ FEMALES $(n=174)$} \\
\hline & \multicolumn{2}{|c|}{$\begin{array}{l}\text { Non-Desaturators } \\
(n=149)(65 \%)\end{array}$} & \multicolumn{2}{|c|}{$\begin{array}{l}\text { Desaturators } \\
(n=79)(35 \%)\end{array}$} & \multicolumn{2}{|c|}{$\begin{array}{l}\text { Non-Desaturators } \\
(n=95)(55 \%)\end{array}$} & \multicolumn{2}{|c|}{$\begin{array}{l}\text { Desaturators } \\
(n=79)(45 \%)\end{array}$} \\
\hline & 65.1 & \pm 8.0 & 66.1 & \pm 7.8 & 62.5 & \pm 8.5 & 63.1 & \pm 7.3 \\
\hline $\mathrm{BMI}, \mathrm{kg} / \mathrm{m}^{2}$ & 26.1 & \pm 5.3 & 25.3 & \pm 5.6 & 26.3 & \pm 6.2 & 24.3 & $\pm 4.8^{*}$ \\
\hline $\mathrm{FM}, \mathrm{kg}$ & 22.1 & \pm 10.2 & 20.9 & \pm 9.9 & 26.4 & \pm 11.6 & 24.0 & \pm 9.4 \\
\hline FFMI, $\mathrm{kg} / \mathrm{m}^{2}$ & 18.5 & \pm 1.9 & 17.9 & \pm 2.2 & 16.0 & \pm 2.0 & 15.3 & \pm 1.7 \\
\hline $\mathrm{FEV}_{1}, \mathrm{~L}$ & 1.7 & \pm 0.7 & 1.3 & $\pm 0.5^{*}$ & 1.4 & \pm 0.4 & 0.9 & $\pm 0.3^{*}$ \\
\hline $\mathrm{FEV}_{1}, \%$ pred. & 55.9 & \pm 19.0 & 40.9 & $\pm 14.7^{*}$ & 61.5 & \pm 16.8 & 42.0 & $\pm 14.5^{*}$ \\
\hline $\mathrm{FEV}_{1} / \mathrm{FVC}, \%$ & 42.7 & \pm 12.4 & 33.0 & $\pm 10.2^{*}$ & 47.1 & \pm 10.1 & 34.3 & $\pm 8.7^{*}$ \\
\hline FVC, \%pred. & 97.8 & \pm 20.8 & 93.2 & \pm 22.0 & 106.6 & \pm 21.7 & 99.4 & \pm 20.6 \\
\hline FRC, \% pred. & 134.5 & \pm 33.9 & 153.2 & $\pm 33.0^{*}$ & 134.8 & \pm 27.9 & 162.6 & $\pm 32.5^{*}$ \\
\hline TLC, \% pred. & 111.2 & \pm 16.3 & 115.6 & $\pm 16.3^{*}$ & 115.5 & \pm 16.3 & 125.2 & $\pm 16.9^{*}$ \\
\hline DLCO, \% pred. & 61.3 & \pm 22.0 & 45.0 & $\pm 15.4^{*}$ & 59.2 & \pm 16.2 & 42.4 & $\pm 13.4^{*}$ \\
\hline $\mathrm{PaO}_{2}, \mathrm{kPa}$ & 10.6 & \pm 1.6 & 9.2 & $\pm 1.3^{*}$ & 10.4 & \pm 1.5 & 9.5 & $\pm 1.6^{*}$ \\
\hline $\mathrm{PaCO}_{2}, \mathrm{kPa}$ & 4.9 & \pm 0.5 & 5.1 & \pm 0.7 & 4.9 & \pm 0.5 & 5.3 & $\pm 0.7^{*}$ \\
\hline $\mathrm{SpO}_{2}, \%$ & 95.2 & \pm 1.9 & 92.8 & $\pm 2.3^{*}$ & 95.6 & \pm 1.9 & 93.7 & $\pm 2.5^{*}$ \\
\hline 6MWD, m & 464.1 & \pm 108.7 & 454.2 & \pm 104.7 & 450.5 & \pm 98.7 & 436.4 & \pm 95.2 \\
\hline 6MWD, \%pred. & 69.0 & \pm 15.6 & 67.9 & \pm 16.3 & 76.1 & \pm 15.0 & 70.5 & \pm 18.1 \\
\hline$M R C$, index & 3.2 & \pm 0.9 & 3.3 & \pm 1.0 & 3.2 & \pm 0.9 & 3.3 & \pm 0.9 \\
\hline
\end{tabular}

Total population of non-hypoxemic patients with COPD categorized by gender and grouped based on the presence of EID to nadir- $\mathrm{SpO}_{2} \leq 88 \%$. Data are mean $\pm \mathrm{SD}$ unless specified otherwise. Asterisk denotes significant differences $(\mathrm{P} \leq 0.05)$ between groups of patients with and without EID. Abbreviations: BMI: Body Mass Index, FM: Fat Mass, FFMI: Fat-Free Mass Index, FEV 1 : Force Expiratory Volume at 1 sec, FVC: Force Vital Capacity, FRC: Functional Residual Capacity, TLC: Total Lung Capacity, DLCO: Diffusing capacity for carbon monoxide, $\mathrm{PaO}_{2}$ : Partial pressure of arterial oxygen, $\mathrm{PaCO}_{2}$ : Partial pressure of carbon-dioxide, $\mathrm{SpO}$ : Saturation of peripheral Oxygen, 6MWD: Six-minute walk distance, MRC: Medical Research Council dyspnea scale. 


\section{Power Analysis Calculation}

\section{Sample size calculation}

Based on the paper of Knower and colleagues (Knower et al. 2001) with a power of $90 \%$ and a level of significance of 0.01 , we estimated the following sample size:

$N=\left[Z 1-\beta V\left(\left(p_{1}\left(1-p_{1}\right)+p_{2}\left(1-p_{2}\right)\right)+Z 1-\alpha / 2 V p_{\text {gem }}\left(1-p_{\text {gem }}\right)\right]^{2} / d 2\right.$

$\mathrm{N}$ = sample size per Group

$\mathrm{p}_{1}=$ proportion group 1

$\mathrm{p}_{2}=$ proportion group 2

$\mathrm{p}_{\text {gem }}=$ mean of both groups

$\mathrm{d}=$ difference in group proportions

Z $1-\alpha / 2=$ standard normal deviate for two tailed test based on alpha level

Z 1- $\beta=$ standard normal deviate for one tailed test based on beta level

$N=\left[1.282 \vee(0.15(1-0.15)+0.25(1-0.25)+2.326 \vee 0.10(1-0.10)]^{2} /(0.25-0.15)^{2}\right.$

$N=201$ patients per group; a total sample size of $N=402$ 



\section{Chapter}

\section{Transcutaneous carbon-dioxide partial pressure trends during six-minute walk test in patients with very severe COPD}




\section{Chapter 7}

\section{ABSTRACT}

\section{Background}

Transcutaneous carbon-dioxide partial-pressure $\left(\mathrm{T}_{C} \mathrm{PCO}_{2}\right)$ can be reliably measured and may be of clinical relevance in COPD. Changes in $\mathrm{T}_{\mathrm{C}} \mathrm{PCO}_{2}$ and exercise-induced hypercapnia $(\mathrm{EIH})$ during six minute walk test (6MWT) need further investigation. We aimed (1) to define patterns of $\mathrm{T}_{\mathrm{C}} \mathrm{PCO}_{2}$ trends during 6MWT and (2) to study determinants of $\mathrm{CO}_{2}$-retention and $\mathrm{EIH}$.

\section{Methods}

Sixty-two COPD patients (age: $63 \pm 8$ years, $\mathrm{FEV}_{1}$ : $33 \pm 10 \%$ predicted) were recruited and $\mathrm{T}_{\mathrm{C}} \mathrm{PCO}_{2}$ was recorded by SenTec digital-monitoring-system during 6MWT.

\section{Results}

Half of patients (50\%) exhibited $\mathrm{CO}_{2}$-retention $\left(\mathrm{T}_{\mathrm{C}} \mathrm{PCO}_{2}[\Delta]>4 \mathrm{mmHg}\right) ; 26 \%$ preserved and $24 \%$ reduced $\mathrm{T}_{\mathrm{C}} \mathrm{PCO}_{2}$. Nineteen (31\%) patients presented $\mathrm{EIH}\left(\mathrm{T}_{\mathrm{C}} \mathrm{PCO}_{2}>45 \mathrm{mmHg}\right)$. $\mathrm{EIH}$ was associated to higher baseline- $\mathrm{P}_{\mathrm{C}} \mathrm{CO}_{2}$, worse $\mathrm{FEV} \mathrm{V}_{1}$, lower inspiratory-pressures, underweight/normal $\mathrm{BMI}$, and pre-walk dyspnea. Stronger determinants of $\mathrm{CO}_{2^{-}}$ retention were $\mathrm{FEV}_{1}$ and pre-walk dyspnea, whereas baseline- $\mathrm{P}_{\mathrm{C}} \mathrm{CO}_{2}$ and pre-walk dyspnea better predict EIH.

\section{Conclusions}

$\mathrm{PCO}_{2}$ response to $6 \mathrm{MWT}$ is highly heterogeneous; however, very low $\mathrm{FEV}_{1}$ and elevated baseline- $\mathrm{P}_{\mathrm{C}} \mathrm{CO}_{2}$ together with pre-walk dyspnea increase the risk for $\mathrm{CO}_{2}$-retention and $\mathrm{EIH}$. Overweight BMI seems to carry a protective effect against EIH in very severe COPD. 


\section{INTRODUCTION}

Six-minute walk test (6MWT) is widely used to assess functional exercise performance and prognosis in patients with chronic obstructive pulmonary disease (COPD). ${ }^{1-3}$ Moreover, the 6MWT is used as field test to assess exercise-induced oxygen desaturation ${ }^{4,5}$ which has an additional prognostic value besides the 6-min walk distance (6MWD). ${ }^{6,7}$ Also, transcutaneous carbon-dioxide pressure $\left(\mathrm{T}_{C} \mathrm{PCO}_{2}\right)$ can be measured reliably as an indicator of ventilatory adequacy and may be of clinical relevance in patients with COPD. ${ }^{8-11}$

Resting $\mathrm{PCO}_{2}$ is highly variable amongst patients with COPD. ${ }^{12}$ Respiratory mechanics impairments, reduced ventilation (hypoventilation), hyperinflation, and reduced gas exchange capabilities (ventilation / perfusion mismatch) may account for $\mathrm{CO}_{2}$ retention and often lead to hypercapnia. ${ }^{13-16}$ During exercise, a substantial $\mathrm{CO}_{2}$ retention $\left(\mathrm{T}_{\mathrm{C}} \mathrm{PCO}_{2}\right.$ $[\Delta]>4 \mathrm{mmHg}$ from baseline) may also occur frequently in patients with COPD and can result in exercise-induced hypercapnia $\left(\mathrm{EIH}\right.$; defined by an elevation of $\mathrm{PCO}_{2}$ levels greater than $45 \mathrm{mmHg} / 6.0 \mathrm{kpa}) .^{15,16}$

Indeed, exercise-induced changes in $\mathrm{PCO}_{2}$ are highly dependent upon several pathophysiological mechanisms in COPD. ${ }^{16,17}$ Even though many COPD patients are normocapnic at rest, some patients may exhibit substantial $\mathrm{CO}_{2}$ retention during exercise, which often results in $\mathrm{EIH}$ and it is associated with a greater tendency toward respiratory failure. ${ }^{18,19}$ In contrast, other patients with COPD may significantly reduce $\mathrm{PCO}_{2}$ on exertion even to hypocapnic levels and be at risk of acute respiratory alkalosis due to hyperventilation. ${ }^{20}$ Indeed, levels of $\mathrm{PCO}_{2}$ have been reported as independent prognostic factor with a U-shaped association with mortality in COPD. ${ }^{21}$

A decline of $\mathrm{PCO}_{2}$ levels is less common than $\mathrm{CO}_{2}$ retention and the occurrence of $\mathrm{EIH}$ in very severe COPD. Although, EIH has been proposed as an indication for the progress of disease severity regarding resting hypercapnia ${ }^{18}$ and also determines a condition of acute respiratory failure, which is related to high risk for death in patients with advanced $\mathrm{COPD}^{22}$, the current literature provides only scarce information on $\mathrm{CO}_{2}$ responses at cycle ergometer test ${ }^{16,23,24}$ or $6 \mathrm{MWT}{ }^{25}$ To date, the determinants of $\mathrm{CO}_{2}$ retention and $\mathrm{EIH}$ have never been investigated using continuous recording of $\mathrm{T}_{\mathrm{C}} \mathrm{PCO}_{2}$ during the 6MWT in patients with very severe COPD.

Herein, we report an analysis of $\mathrm{CO}_{2}$ response to exercise during the 6MWT in patients with very severe COPD. We aimed to (1) to define patterns of $\mathrm{T}_{\mathrm{C}} \mathrm{PCO}_{2}$ trends during the 6MWT and (2) to study the determinants of $\mathrm{CO}_{2}$ retention and the occurrence of EIH during walking in very severe COPD. 


\section{METHODS}

\section{Design and participants}

Patients aged 30 to 75 years with very severe COPD (GOLD IV), with and without the presence of chronic respiratory failure, were recruited prospectively. Exclusion criteria were resting capillary $\mathrm{P}_{\mathrm{C}} \mathrm{CO}_{2}>55 \mathrm{mmHg}$, use of non-invasive ventilation, and/or acute exacerbation in the last 4 weeks. The Bavarian ethical committee approved this study and all subjects provided written informed consent (Germany: Ethic committee approval number: 12007). Sixty-two ( $n=62$ ) out of 75 patients with very severe COPD, participating in an inpatient Pulmonary Rehabilitation at Schoen Klinik Berchtesgadener Land (Schoenau am Koenigssee, Germany) were used for data analyses. Data from 13 patients were incomplete, and, in turn, not used for further analyses.

\section{Assessments}

All patients underwent physical examination, anthropometric and lung function measurements. Capillary blood gases (CBG) at rest were obtained under normal ambient condition and a complete medical history was recorded. Lung function was assessed by post-bronchodilator spirometry, plethysmographic lung volumes at rest (i.e. tidal volume [Vt], maximal inspiratory mouth pressure [Pi max], expired ventilation $[V E])$, measures of carbon monoxide diffusing capacity (DLCO), and CBG analysis $\left(\mathrm{P}_{C} \mathrm{O}_{2}\right.$, $\left.\mathrm{P}_{\mathrm{C}} \mathrm{CO}_{2}\right)$. Body mass index $\left(\mathrm{BMI}, \mathrm{kg} / \mathrm{m}^{2}\right)$ was defined as weight divided by squared height, and fat mass index (FMI, $\mathrm{kg} / \mathrm{m}^{2}$ ) was calculated as the fat mass ( $\mathrm{kg}$ ) divided by the squared height $\left(\mathrm{m}^{2}\right)$. Fat mass and fat-free mass were measured using bioelectrical impedance (NutriGuard MS, Poecking, Germany). Patients completed the selfadministered and validated version of the Hospital Anxiety and Depression Scale $(\mathrm{HADS})^{26}$ and the Chronic Respiratory Questionnaire (CRQ) ${ }^{27}$.

Two 6MWTs were performed one week apart according to the international guidelines. ${ }^{1,2}$ The test with the longest walking distance was used for further analysis. Participants were asked to walk indoors in a flat, straight, $30 \mathrm{~m}$ walking course supervised by a well-trained health-care professional. Patients were encouraged using standard methodology every minute of the $6 \mathrm{MWT}$. ${ }^{1}$ They were allowed to stop and rest during the test, but were instructed to resume walking as soon as possible. Oxygen saturation $\left(\mathrm{SpO}_{2}\right)$, heart rate $(\mathrm{HR})$ and transcutaneous $\mathrm{CO}_{2}$ levels $\left(\mathrm{T}_{\mathrm{C}} \mathrm{PCO} \mathrm{C}_{2}\right)$ as surrogate for arterial carbon-dioxide partial pressure $\left(\mathrm{PaCO}_{2}\right)^{28}$ were measured continuously using the SenTec digital-monitoring system (Sentec AG, Therwill, Switzerland) carried by a well-trained health-care professional. ${ }^{8}$ Perceived dyspnea and leg fatigue of patients at the 6MWT were quantified by a 10 -scale Schoen Klinik index (modified Borg scale) before and at the end of the exertion (see supplemental materials - Table S1). Exercise- 
induced desaturation (EID) was defined as $\mathrm{SpO}_{2}$ nadir $\leq 88 \%$. Major changes in $\mathrm{T}_{C} \mathrm{PCO}_{2}$ $\left(\mathrm{T}_{\mathrm{C}} \mathrm{PCO}_{2},[\Delta]>4 \mathrm{mmHg}\right)$ during the $6 \mathrm{MWT}$ defined patients as "non-isocapnic" including those who presented either a decreasing $\mathrm{CO}_{2}$ pattern (decline of $\mathrm{T}_{\mathrm{C}} \mathrm{PCO}_{2}>4 \mathrm{mmHg}$ ) or an increasing $\mathrm{CO}_{2}$ pattern (increase of $\mathrm{T}_{\mathrm{C}} \mathrm{PCO}_{2}>4 \mathrm{mmHg}$ ) at exertion. Moreover, the non-isocapnic patients who substantially increased $\mathrm{T}_{C} \mathrm{PCO}_{2}\left(\mathrm{~T}_{C} \mathrm{PCO}_{2},[\Delta]>4 \mathrm{mmHg}\right.$ from baseline) were characterized as " $\mathrm{CO}_{2}$ retainers". Exercise-induced hypercapnia (EIH) was considered as the peak of $\mathrm{T}_{\mathrm{C}} \mathrm{PCO}_{2}>45 \mathrm{mmHg}$ during the $6 \mathrm{MWT}$.

\section{Statistical analysis}

Results are expressed as mean (standard deviation) or proportion (\%). The statistical significance of differences between groups was assessed by analysis of variance, paired T-tests and Chi-square, as appropriate. Pearson's correlation coefficients were used to assess bivariate relationship between changes of carbon dioxide pressure $\left(\mathrm{T}_{\mathrm{C}} \mathrm{PCO}_{2},[\Delta]\right)$ during the 6MWT, and physiological characteristics. Spearman's rank correlation was used to determine the repeatability of the $\mathrm{T}_{\mathrm{C}} \mathrm{PCO}_{2}$ trends (Patterns: Reducing, Isocapnic, Increasing) at 6MWT. Binary logistical regression assessed individual predictors of $\mathrm{CO}_{2}$ retention and $\mathrm{EIH}$. An adjusted logistic regression model was used to estimate odds ratio for $\mathrm{CO}_{2}$ retention and $\mathrm{EIH}$. Receiver operating characteristics (ROC) curves were used to determine threshold values to predict EIH. The Area Under the curve (AUC) was calculated by the trapezoidal rule and the confidence intervals of the AUC was computed by the method of DeLong. ${ }^{29}$ Two-sided level of significance was set at $P<0.05$. Statistical analyses were carried out using the software of MedCalc v.12 and SPSS v.19.0.

\section{RESULTS}

\section{Patient characteristics}

A total of 62 patients with very severe COPD (31\% women) was included in the study. In general, patients had a normal BMI, very severe airflow limitation, static hyperinflation (defined as ratio of residual volume to total lung capacity (RV/TLC >60\%) and very severely impaired lung diffusion capacity (DLCO $\leq 30 \%$ ). They were characterized by impaired respiratory pattern at rest (i.e. small Vt and/or high VE/min) and respiratory muscle weakness (determined by $\mathrm{Pi}$ max $<60 \%$ ), while had low $\mathrm{PcO}_{2}$ levels but normal range in $\mathrm{P}_{\mathrm{C}} \mathrm{CO}_{2}$ as obtained by $\mathrm{CBG}$ analyses. Patients also had impaired health and mood status (rated by CRQ and HADS, respectively) and the vast majority (81\%) was receiving long-term oxygen therapy (LTOT; Table 1 ). 


\section{Chapter 7}

On the 6MWT, patients demonstrated poor exercise capacity (mean 6MWD $<360 \mathrm{~m}$ ) with a substantial exercise-induced $\mathrm{SpO}_{2}$ decline $\left(\mathrm{SpO}_{2} \%[\Delta]=-8\right.$ points), mild elevation in heart rate $(\mathrm{HR}[\Delta]=26 \mathrm{~b} / \mathrm{min})$ and a total small increase in $\mathrm{T}_{C} \mathrm{PCO}_{2}\left(\mathrm{~T}_{C} \mathrm{PCO}_{2},[\Delta]=1.8\right.$ $\mathrm{mmHg}$ ). Patients also reported moderate post-walk dyspnea and mild leg-fatigue at the end of test. Thirty-eight patients (61\%) exhibit EID, 19 patients (31\%) presented EIH, and 13 patients (21\%) had both (Table 1 ).

Table 1. Baseline and exercise characteristics

\begin{tabular}{|c|c|c|c|}
\hline $\begin{array}{l}\text { Baseline } \\
\text { Characteristics }\end{array}$ & $\begin{array}{l}\text { All Patients } \\
(n=62)\end{array}$ & $\begin{array}{l}\text { Exercise } \\
\text { Characteristics }\end{array}$ & $\begin{array}{l}\text { All Patients } \\
(\mathrm{n}=62)\end{array}$ \\
\hline Women, \#, (\%) & 19(31) & 6MWD, m & $358 \pm 97$ \\
\hline Age, years & $63.0 \pm 8.1$ & 6MWD, \%predicted & $53.3 \pm 14.4$ \\
\hline $\mathrm{BMI}, \mathrm{kg} / \mathrm{m}^{2}$ & $24.2 \pm 4.6$ & $\mathrm{SpO}_{2}$ pre, $\%$ & $95.9 \pm 2.7$ \\
\hline FMI, kg & $5.6 \pm 2.7$ & $\mathrm{SpO}_{2}$ post, $\%$ & $88.0 \pm 6.8$ \\
\hline FFMI, $\mathrm{kg} / \mathrm{m}^{2}$ & $18.6 \pm 2.8$ & $\mathrm{SpO}_{2}[\Delta], \%$ & $-7.9 \pm 5.5$ \\
\hline $\mathrm{FEV}_{1}, \%$ pred. & $33.2 \pm 10.1$ & HR pre, b/min & $85 \pm 14$ \\
\hline RV/TLC, \% & $69.1 \pm 8.8$ & HR post, $\mathrm{b} / \mathrm{min}$ & $112 \pm 17$ \\
\hline DLCO, \%pred. & $30.1 \pm 16.8$ & $\mathrm{HR}[\Delta], \mathrm{b} / \mathrm{min}$ & $26 \pm 10$ \\
\hline$V t, L$ & $0.96 \pm 0.2$ & $\mathrm{~T}_{\mathrm{c}} \mathrm{PCO}_{2}$ pre, $\mathrm{mmHg}$ & $40.9 \pm 6.0$ \\
\hline $\mathrm{Bf}, \mathrm{br} / \mathrm{min}$ & $21.0 \pm 6.5$ & $\mathrm{~T}_{\mathrm{c}} \mathrm{PCO}_{2}$ post, $\mathrm{mmHg}$ & $42.7 \pm 7.5$ \\
\hline VE, L/min & $19.4 \pm 5.5$ & $\mathrm{~T}_{\mathrm{c}} \mathrm{PCO}_{2}[\Delta], \mathrm{mmHg}$ & $1.8 \pm 6.0$ \\
\hline Pi max, \%pred. & $59.4 \pm 18.9$ & Dyspnea pre, index & $1.7 \pm 1.9$ \\
\hline $\mathrm{P}_{\mathrm{c}} \mathrm{O}_{2}, \mathrm{mmHg}$ & $59.4 \pm 8.2$ & Dyspnea post, index & $5.5 \pm 2.6$ \\
\hline $\mathrm{P}_{\mathrm{c}} \mathrm{CO}_{2}, \mathrm{mmHg}$ & $40.8 \pm 5.1$ & Dyspnea $[\Delta]$, index & $3.8 \pm 2.4$ \\
\hline CRQ Dyspnea, score & $3.3 \pm 1.5$ & Fatigue pre, index & $1.4 \pm 1.9$ \\
\hline CRQ Fatigue, score & $4.4 \pm 1.4$ & Fatigue post, index & $3.7 \pm 2.6$ \\
\hline CRQ Emotion F., score & $4.3 \pm 1.5$ & Fatigue $[\Delta]$, index & $2.3 \pm 2.2$ \\
\hline CRQ Mastery, score & $4.1 \pm 1.5$ & & \\
\hline CRQ Total score & $4.0 \pm 1.3$ & EID, $n,(\%)$ & $38(61)$ \\
\hline HADS Anxiety, score & $6.0 \pm 3.9$ & $\mathrm{EIH}$ & $19(31)$ \\
\hline HADS Depression, score & $6.3 \pm 4.1$ & $E I D \& E I H$ & $13(21)$ \\
\hline LTOT, n, (\%) & $50(81)$ & Oxygen users & $49(79)$ \\
\hline
\end{tabular}

Abbreviations: BMI: Body Mass Index, FMI: Fat Mass Index, FFMI: Fat-free Mass Index, FEV ${ }_{1}$ : Force Expiratory Volume at 1sec, RV/TLC: Residual Volume / Total Lung Capacity ratio, DLCO: Diffusing capacity for carbon monoxide, Vt: tidal volume, Bf: Breathing frequency, VE: Expired ventilation, Pi max: Maximal inspiratory mouth pressure; $\mathrm{P}_{C} \mathrm{O}_{2}$ : Capillary oxygen partial pressure, $\mathrm{P}_{C} \mathrm{CO}_{2}$ : Capillary carbon-dioxide partial pressure, $\mathrm{CRQ}$ : Chronic Respiratory Disease Questionnaire, HADS: Hospital Anxiety and Depression Scale, LTOT: Long-Term Oxygen Therapy, 6MWD: Six-minute walk distance, $\mathrm{SpO}_{2}$ : Saturation of peripheral Oxygen, HR: Heart Rate, $\mathrm{T}_{\mathrm{C}} \mathrm{PCO}_{2}$ : Transcutaneous carbon-dioxide partial pressure, EID: Exercise-induced desaturation, ElH: Exerciseinduced hypercapnia. Predicted values of pulmonary measurements were estimated according to European Community for Coal and Steel (ECCS) report 1993 as adopted by Quanjer PhH et al. ERJ 1993; 16:5-40. Predicted values of 6MWD\% were calculated according to Troosters T. et al. ERJ 1999; 14:270-4. 


\section{Exercise-induced carbon dioxide retention during 6MWT}

The $\mathrm{T}_{\mathrm{C}} \mathrm{PCO}_{2}$ response to exercise was highly heterogeneous during the 6MWT. Half of patients (50\%) increased substantially $\mathrm{T}_{C} \mathrm{PCO}_{2}$ levels $\left(\mathrm{TCPCO}_{2}[\Delta]>4 \mathrm{mmHg}\right.$; " $\mathrm{CO}_{2}$ retainers"), while the rest either maintained or even decreased $\mathrm{CO}_{2}$ at exertion (" $\mathrm{CO}_{2}$ non-retainers"). From all patients, $\mathrm{CO}_{2}$ non-retainers consisted of 16 patients (26\%) who preserved the $\mathrm{CO}_{2}$ levels $\left(\mathrm{T}_{\mathrm{C}} \mathrm{PCO}_{2}[\Delta]\right.$ ranged from -4 to $4 \mathrm{mmHg}$; "Isocapnic $\mathrm{T}_{\mathrm{C}} \mathrm{PCO}_{2^{-}}$ pattern"); and 15 patients (24\%) who reduced the $\mathrm{CO}_{2}$ levels $\left(\mathrm{T}_{C} \mathrm{PCO}_{2}[\Delta]\right.$ decline $>4$ $\mathrm{mmHg}$; "decreasing $\mathrm{T}_{\mathrm{C}} \mathrm{PCO}_{2}$-pattern") during the 6MWT (Supplementary Figures S1-S2 and Table 2).

Table 2. Transcutaneous measurements during the 6MWT by SenTec digital-monitoring system

\begin{tabular}{|c|c|c|c|c|}
\hline \multirow[b]{2}{*}{ Variables } & \multirow[b]{2}{*}{$\begin{array}{l}\text { Total Population } \\
(n=62)\end{array}$} & \multicolumn{2}{|c|}{$\mathrm{CO}_{2}$ non-retainers } & \multirow{2}{*}{$\begin{array}{l}\mathrm{CO}_{2} \text { retainers } \\
\text { Increasing } \\
\mathrm{CO}_{2} \text { pattern } \\
(\mathrm{n}=31,50 \%)\end{array}$} \\
\hline & & $\begin{array}{l}\text { Decreasing } \\
\mathrm{CO}_{2} \text { pattern } \\
(\mathrm{n}=15,24 \%)\end{array}$ & $\begin{array}{l}\text { Isocapnic } \\
\mathrm{CO}_{2} \text { pattern } \\
(\mathrm{n}=16,26 \%)\end{array}$ & \\
\hline$\overline{\mathrm{T}_{\mathrm{C}} \mathrm{PCO}_{2} \text { pre, } \mathrm{mmHg}}$ & $40.9 \pm 6.0$ & $42.6 \pm 5.3$ & $40.5 \pm 5.8$ & $40.2 \pm 6.4$ \\
\hline $\mathrm{T}_{\mathrm{C}} \mathrm{PCO}_{2}$ post, $\mathrm{mmHg}$ & $42.7 \pm 7.5$ & $36.7 \pm 5.9$ & $40.8 \pm 5.6$ & $46.6 \pm 6.9$ \\
\hline $\mathrm{T}_{\mathrm{C}} \mathrm{PCO}_{2}[\Delta], \mathrm{mmHg}$ & $1.8 \pm 6.0$ & $-6.0 \pm 3.0$ & $0.2 \pm 1.5$ & $6.4 \pm 3.9$ \\
\hline $\mathrm{T}_{\mathrm{C}} \mathrm{PCO}_{2}$ nadir, $\mathrm{mmHg}$ & $38.5 \pm 6.4$ & $35.5 \pm 5.9$ & $39.4 \pm 5.7$ & $39.4 \pm 6.7$ \\
\hline $\mathrm{T}_{\mathrm{C}} \mathrm{PCO}{ }_{2}$ peak, $\mathrm{mmHg}$ & $44.8 \pm 6.8$ & $42.9 \pm 5.4$ & $41.7 \pm 5.6$ & $47.4 \pm 7.1$ \\
\hline $\mathrm{T}_{\mathrm{C}} \mathrm{PCO}_{2}[\Delta], \mathrm{mmHg}$ & $2.3 \pm 7.2$ & $-7.4 \pm 3.1$ & $0.5 \pm 2.5$ & $7.9 \pm 3.9$ \\
\hline $\mathrm{SpO}_{2} \%$, pre & $95.9 \pm 2.7$ & $95.8 \pm 2.8$ & $96.0 \pm 3.1$ & $96.0 \pm 2.5$ \\
\hline $\mathrm{SpO}_{2} \%$, nadir & $86.1 \pm 7.1$ & $88.3 \pm 6.2$ & $85.4 \pm 8.4$ & $85.4 \pm 6.8$ \\
\hline $\mathrm{SpO}_{2}[\Delta], \%$ & $-9.8 \pm 5.8$ & $-7.5 \pm 4.4$ & $-10.6 \pm 7.3$ & $-10.6 \pm 5.3$ \\
\hline HR pre, b/min & $85.9 \pm 14.4$ & $85.9 \pm 15.3$ & $82.8 \pm 12.5$ & $87.5 \pm 15.0$ \\
\hline HR peak, b/min & $115.1 \pm 17.7$ & $113.3 \pm 15.3$ & $115.1 \pm 18.1$ & $115.9 \pm 19.1$ \\
\hline $\mathrm{HR}[\Delta], \mathrm{b} / \mathrm{min}$ & $29.2 \pm 11.0$ & $27.4 \pm 5.3$ & $32.3 \pm 13.1$ & $28.5 \pm 11.8$ \\
\hline EID, $n,(\%)$ & $38(61)$ & $8(53)$ & $9(56)$ & $21(68)$ \\
\hline $\mathrm{EIH}$ & 19(31) & $0(0)$ & $0(0)$ & 19(61) \\
\hline$E I D \& E I H$ & $13(21)$ & $0(0)$ & $0(0)$ & $13(42)$ \\
\hline
\end{tabular}

Abbreviations: $\mathrm{T}_{\mathrm{C}} \mathrm{PCO}_{2}$ : Transcutaneous carbon-dioxide partial pressure; $\mathrm{SpO}_{2}$ : Saturation of peripheral oxygen, HR: heart rate; EID: exercise-induced oxygen desaturation, EIH: exercise-induced hypercapnia. The $\mathrm{CO}_{2}$ patterns were defined according to $\mathrm{T}_{\mathrm{C}} \mathrm{PCO}_{2}$ trends $([\Delta]>4 \mathrm{mmHg})$ during $6 \mathrm{MWT}$. Predicted values of pulmonary measurements were estimated according to European Community for Coal and Steel (ECCS) report 1993 as adopted by Quanjer PhH et al. Eur Respir J Suppl 1993, suppl. 6. Predicted values of 6MWD\% were calculated according to Troosters et al. Eur Respir J 1999; 14:270-4.

Age, $\mathrm{BMI}$, the degree of airflow limitation $\left(\mathrm{FEV}_{1}\right), \mathrm{CO}_{2}$ transfer factor as defined by DLCO, respiratory muscle weakness as determined by $\mathrm{Pi}$ max, baseline $\mathrm{P}_{C} \mathrm{O}_{2}$, and the proportion of LTOT users were similar between patients with and without $\mathrm{CO}_{2}$ retention during the 6MWT (Table 3). The $\mathrm{CO}_{2}$ retainers had a better health status as well as a better mood status (Table 3). 


\section{Chapter 7}

Nineteen (31\%) out of 62 patients presented $\mathrm{EIH}\left(\mathrm{T}_{\mathrm{C}} \mathrm{PCO}_{2}>45 \mathrm{mmHg}\right)$. Patients with $\mathrm{EIH}$ had lower BMI, lower $\mathrm{FEV}_{1}$, smaller tidal volume resulting in lower ventilation, greater respiratory muscle weakness (as defined by $\mathrm{Pi}$ max) and higher baseline $\mathrm{P}_{\mathrm{C}} \mathrm{CO}_{2}$ compared to non-EIH patients (Table 3). $\mathrm{CO}_{2}$ retention or EIH during the 6MWT did not affect the exercise capacity (6MWD) in patients with very severe COPD, even though patient with EIH had higher pre-walk dyspnea compared to non-EIH (Table 4). Moreover, lowest oxygen saturation $\left(\mathrm{SpO}_{2}\right.$ nadir) and peak heart rate do not seem to be related with $\mathrm{T}_{\mathrm{C}} \mathrm{PCO}_{2}$ responses during the 6MWT (Table 4 and Figure 1).

Table 3. Baseline characteristics and categorization according to $\mathrm{CO}_{2}$ retention and $\mathrm{EIH}$

\begin{tabular}{|c|c|c|c|c|}
\hline $\begin{array}{l}\text { Baseline } \\
\text { Characteristics }\end{array}$ & $\begin{array}{l}\mathrm{CO}_{2} \text { non-retainers } \\
(\mathrm{n}=31,50 \%)\end{array}$ & $\begin{array}{l}\mathrm{CO}_{2} \text { retainers } \\
(n=31,50 \%)\end{array}$ & $\begin{array}{l}\text { Non-ElH } \\
(n=43,69 \%)\end{array}$ & $\begin{array}{l}\text { EIH } \\
(n=19,31 \%)\end{array}$ \\
\hline Women, n, (\%) & $10(32)$ & $9(29)$ & $13(30)$ & $6(32)$ \\
\hline Age, years, $\pm S D$ & $62.9 \pm 8.6$ & $63.0 \pm 7.6$ & $62.6 \pm 8.4$ & $63.8 \pm 7.4$ \\
\hline $\mathrm{BMI}, \mathrm{kg} / \mathrm{m}^{2}$ & $25.2 \pm 4.9$ & $23.2 \pm 4.2$ & $25.0 \pm 4.4$ & $22.3 \pm 4.9 *$ \\
\hline $\mathrm{FMI}, \mathrm{kg} / \mathrm{m}^{2}$ & $6.2 \pm 3.1$ & $5.1 \pm 2.3$ & $6.0 \pm 2.9$ & $4.8 \pm 2.3$ \\
\hline FFMI, $\mathrm{kg} / \mathrm{m}^{2}$ & $19.0 \pm 3.0$ & $18.2 \pm 2.6$ & $19.0 \pm 2.6$ & $17.7 \pm 3.0$ \\
\hline $\mathrm{FEV}_{1}, \%$ pred. & $35.0 \pm 11.2$ & $31.3 \pm 8.6$ & $34.8 \pm 10.8$ & $29.5 \pm 7.4^{*}$ \\
\hline RV/TLC, \% & $68.5 \pm 9.8$ & $69.8 \pm 7.8$ & $68.1 \pm 8.9$ & $71.4 \pm 8.4$ \\
\hline DLCO, \% pred. & $28.4 \pm 18.6$ & $32.0 \pm 14.6$ & $30.3 \pm 18.0$ & $29.9 \pm 13.9$ \\
\hline$V t, L$ & $0.97 \pm 0.2$ & $0.94 \pm 0.3$ & $1.01 \pm 0.2$ & $0.81 \pm 0.3^{*}$ \\
\hline $\mathrm{Bf}, \mathrm{br} / \mathrm{min}$ & $21.7 \pm 7.6$ & $20.4 \pm 5.1$ & $20.8 \pm 6.8$ & $21.6 \pm 5.8$ \\
\hline$V E, L / \min$ & $20.4 \pm 5.8$ & $18.5 \pm 5.2$ & $20.5 \pm 5.4$ & $17.0 \pm 5.3^{*}$ \\
\hline Pi max, \%pred. & $58.8 \pm 19.6$ & $60.0 \pm 18.4$ & $62.3 \pm 20.0$ & $52.2 \pm 13.7^{*}$ \\
\hline $\mathrm{P}_{\mathrm{C}} \mathrm{O}_{2}, \mathrm{mmHg}$ & $58.9 \pm 9.0$ & $59.8 \pm 7.4$ & $59.7 \pm 8.2$ & $58.7 \pm 8.3$ \\
\hline $\mathrm{P}_{\mathrm{C}} \mathrm{CO}_{2}, \mathrm{mmHg}$ & $40.3 \pm 4.8$ & $41.2 \pm 5.4$ & $39.6 \pm 4.6$ & $43.5 \pm 5.3^{* *}$ \\
\hline CRQ Dyspnea, score & $3.0 \pm 1.3$ & $3.6 \pm 1.6$ & $3.3 \pm 1.5$ & $3.3 \pm 1.4$ \\
\hline CRQ Fatigue, score & $3.9 \pm 1.3$ & $4.9 \pm 1.4^{*}$ & $4.3 \pm 1.4$ & $4.5 \pm 1.5$ \\
\hline CRQ Emotional function score & $3.6 \pm 1.6$ & $4.8 \pm 1.2 * *$ & $4.2 \pm 1.7$ & $4.5 \pm 1.2$ \\
\hline CRQ Mastery, score & $3.5 \pm 1.7$ & $4.6 \pm 1.2^{*}$ & $4.0 \pm 1.7$ & $4.3 \pm 1.1$ \\
\hline CRQ Total score & $3.5 \pm 1.3$ & $4.5 \pm 1.1^{* *}$ & $3.9 \pm 1.4$ & $4.1 \pm 1.0$ \\
\hline HADS Anxiety, score & $7.5 \pm 3.6$ & $4.6 \pm 3.6 * *$ & $6.4 \pm 4.0$ & $5.1 \pm 3.5$ \\
\hline HADS Depression, score & $8.1 \pm 4.5$ & $4.8 \pm 3.0^{* *}$ & $6.7 \pm 4.5$ & $5.5 \pm 3.0$ \\
\hline LTOT, n & $27(87)$ & $23(74)$ & $34(79)$ & $16(84)$ \\
\hline
\end{tabular}

Abbreviations: Please see legend Table 1. Baseline characteristics of patients categorized according to $\mathrm{CO}_{2}$ retention and the occurrence of EIH. Asterisks denote significant difference: $\left({ }^{*}\right) p<0.05,\left({ }^{* *}\right) p<0.01$. 
Table 4. Six-min walk test characteristics and categorization by $\mathrm{CO}_{2}$ retention and $\mathrm{EIH}$

\begin{tabular}{|c|c|c|c|c|}
\hline $\begin{array}{l}6 \mathrm{MWT} \\
\text { Characteristics }\end{array}$ & $\begin{array}{l}\mathrm{CO}_{2} \text { non-retainers } \\
(\mathrm{n}=31,50 \%)\end{array}$ & $\begin{array}{l}\mathrm{CO}_{2} \text { retainers } \\
(\mathrm{n}=31,50 \%)\end{array}$ & $\begin{array}{l}\text { Non-EIH } \\
(n=43,69 \%)\end{array}$ & $\begin{array}{l}\text { ElH } \\
(n=19,31 \%)\end{array}$ \\
\hline 6MWD, m & $343 \pm 99$ & $373 \pm 94$ & $369 \pm 98$ & $334 \pm 92$ \\
\hline 6MWD, \%predicted & $51.3 \pm 14.5$ & $55.3 \pm 14.0$ & $54.6 \pm 14.2$ & $50.3 \pm 14.4$ \\
\hline $\mathrm{SpO}_{2} \%$, pre & $95.9 \pm 2.9$ & $96.0 \pm 2.5$ & $96.0 \pm 2.7$ & $95.8 \pm 2.8$ \\
\hline $\mathrm{SpO}_{2} \%$, nadir & $86.8 \pm 7.5$ & $85.4 \pm 6.8$ & $86.4 \pm 7.4$ & $85.4 \pm 6.7$ \\
\hline HR pre, b/m & $91 \pm 15$ & $94 \pm 13$ & $92 \pm 15$ & $94 \pm 9$ \\
\hline HR peak, b/m & $114 \pm 17$ & $115 \pm 19$ & $115 \pm 19$ & $114 \pm 13$ \\
\hline $\mathrm{T}_{\mathrm{C}} \mathrm{PCO}_{2}$ pre, $\mathrm{mmHg}$ & $41.5 \pm 5.6$ & $40.2 \pm 6.4$ & $39.6 \pm 5.9$ & $43.8 \pm 5.2^{*}$ \\
\hline $\mathrm{T}_{\mathrm{C}} \mathrm{PCO}_{2}$ post, $\mathrm{mmHg}$ & $38.5 \pm 6.0$ & $47.4 \pm 7.1^{* *}$ & $38.9 \pm 5.3$ & $52.0 \pm 4.8^{* *}$ \\
\hline Dyspnea, pre & $1.5 \pm 2.0$ & $2.0 \pm 1.8$ & $1.4 \pm 1.8$ & $2.5 \pm 2.0^{*}$ \\
\hline Dyspnea, post & $5.1 \pm 2.6$ & $6.0 \pm 2.5$ & $5.4 \pm 2.8$ & $5.8 \pm 2.0$ \\
\hline Leg Fatigue, pre & $1.2 \pm 1.8$ & $1.5 \pm 1.9$ & $1.2 \pm 1.8$ & $1.7 \pm 2.2$ \\
\hline Leg Fatigue, post & $3.5 \pm 2.5$ & $3.9 \pm 2.6$ & $3.3 \pm 2.5$ & $4.6 \pm 2.6$ \\
\hline$E I D, n,(\%)$ & $17(55)$ & $21(68)$ & $25(58)$ & $13(68)$ \\
\hline
\end{tabular}

Abbreviations: 6MWD: six-minute walk distance; $\mathrm{SpO}_{2}$ : Saturation of peripheral oxygen; HR: heart rate; $\mathrm{T}_{\mathrm{C}} \mathrm{PCO}_{2}$ : Transcutaneous carbon-dioxide partial-pressure, EID: exercise-induced oxygen desaturation, EIH: exercise-induced hypercapnia. Six-minute walk test characteristics and categorization performed according to $\mathrm{CO}_{2}$ retention and the occurrence of EIH. Asterisks denote significant difference: $\left(^{*}\right) p<0.05,\left({ }^{* *}\right) p<0.01$.

\section{Repeatability of exercise-induced carbon dioxide patterns during 6MWT}

High repeatability of the $\mathrm{T}_{C} \mathrm{PCO}_{2}$ patterns during exercise was observed between the longest 6MWT and the shortest 6MWT for each patient. Specifically, the $73 \%$ of the patients demonstrated an identical $\mathrm{T}_{\mathrm{C}} \mathrm{PCO}_{2}$ pattern in both 6MWT (Spearman's rho=0.60, $p<0.001$ ). In addition, the mean values of the changes in $\mathrm{T}_{\mathrm{C}} \mathrm{PCO}_{2}$ as well as $\mathrm{SpO}_{2}$ and heart rate during both performed 6MWT were similar (Supplementary figure S3). 


\section{Chapter 7}

Table 5. Logistic regression: Determinants of $\mathrm{CO}_{2}$ retention and $\mathrm{EIH}$ in patients with very severe COPD

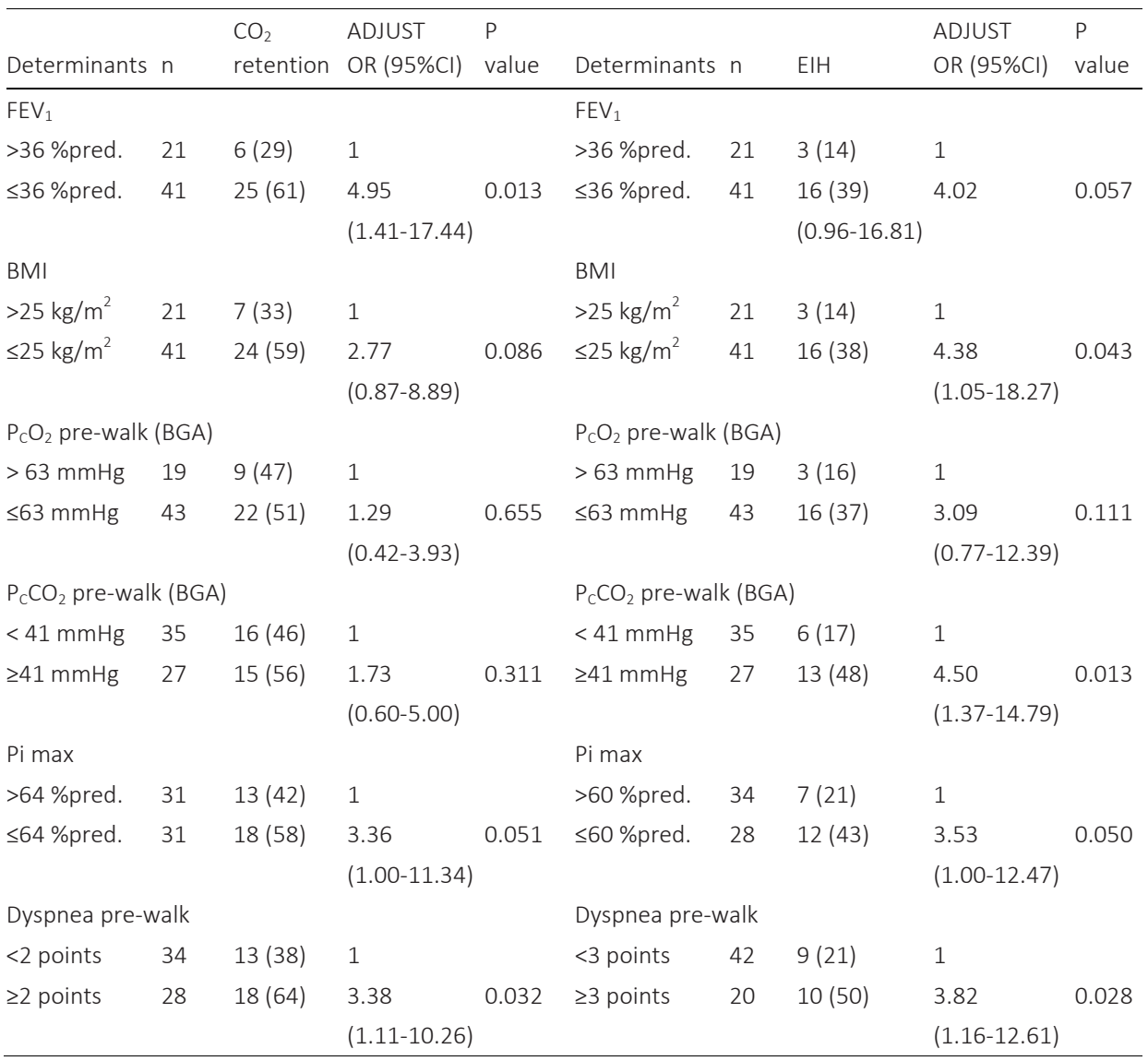

Abbreviations: Please see legend Table 1. A Logistic regression demonstrated the determinants of $\mathrm{CO}_{2}$ retention and EIH during the 6MWT in 62 patients with very severe COPD. Each determinant was adjusted for age ( $\geq 60$ years), sex (females), and Long-Term Oxygen Therapy (LTOT). $\mathrm{CO}_{2}$ retention and EIH were defined as $\mathrm{TCPCO}_{2}[\Delta]>4 \mathrm{mmHg}$ and $\mathrm{TCPCO}_{2}>45 \mathrm{mmHg}$, respectively. Significance was set on $\mathrm{p}<0.05$.

\section{Correlates of $T_{C} P \mathrm{PO}_{2}$ during the $6 \mathrm{MWT}$}

Post-walk $\mathrm{T}_{\mathrm{C}} \mathrm{PCO}_{2}$ was correlated with the levels of capillary $\mathrm{P}_{C} \mathrm{CO}_{2}(r=0.53, p<0.001)$ at rest and had an inverse correlation with $\mathrm{FEV}_{1} \%$ predicted $(\mathrm{r}=-0.32, \mathrm{p}<0.05)$. The delta values $\left(\mathrm{T}_{\mathrm{C}} \mathrm{PCO}_{2},[\Delta]\right)$ were associated only with the $\mathrm{FEV}_{1} \% \quad(r=-0.26, p<0.005$; Supplementary table S2.)

\section{Determinants of $\mathrm{TCPCO}_{2}$ increase and EIH during the 6MWT}

Using receiver operating characteristics (ROC) curves, the threshold values with the best sensitivity and specificity to predict $\mathrm{T}_{\mathrm{C}} \mathrm{PCO}_{2}$ retention and $\mathrm{EIH}$ were determined for $\mathrm{BMI}$ 
$\left(\leq 24 \mathrm{~kg} / \mathrm{m}^{2}\right), \mathrm{FEV}_{1}\left(\leq 36 \%\right.$ pred.), baseline $\mathrm{P}_{\mathrm{C}} \mathrm{O}_{2}(\leq 63 \mathrm{mmHg})$, baseline $\mathrm{P}_{\mathrm{C}} \mathrm{CO}_{2}(\geq 41 \mathrm{mmHg})$, Pi $\max \left(\leq 64 \%\right.$ pred. - $\mathrm{CO}_{2}$ retainers/ $\leq 60 \%$ pred. - EIH) and pre-walk dyspnea (Schoen Klinik scale: $\geq 2$ points - $\mathrm{CO}_{2}$ retainers/ $\geq 3$ points - ElH; Supplementary tables S3-S4).

Using logistic regression of these ROC curve identified cut-offs, showed that $\mathrm{FEV}_{1}$ $\leq 36 \%$ pred. and higher pre-walk dyspnea ( $\geq 2$ points) were determinants of $\mathrm{CO}_{2}$ retention while $\mathrm{P}_{\mathrm{C}} \mathrm{CO}_{2} \geq 41 \mathrm{mmHg}$ and higher pre-walk dyspnea ( $\geq 3$ points) were determinants of EIH after adjustment for age ( $\geq 60$ years), sex (females), and LTOT (Table 5). Interestingly, a higher BMI $\left(>25 \mathrm{~kg} / \mathrm{m}^{2}\right)$ seems to carry a protective effect against the occurrence of EIH in advanced COPD.

\section{A. $\mathrm{CO}_{2}$ non-retainers}

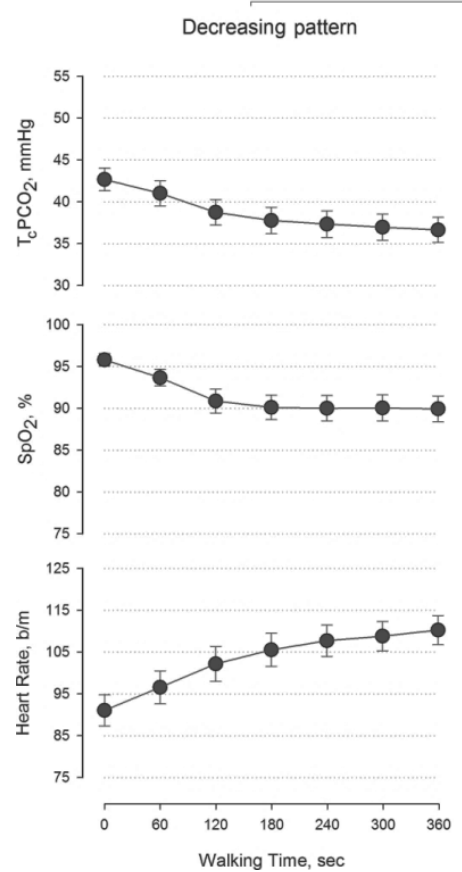

B. $\mathrm{CO}_{2}$ retainers

Increasing pattern
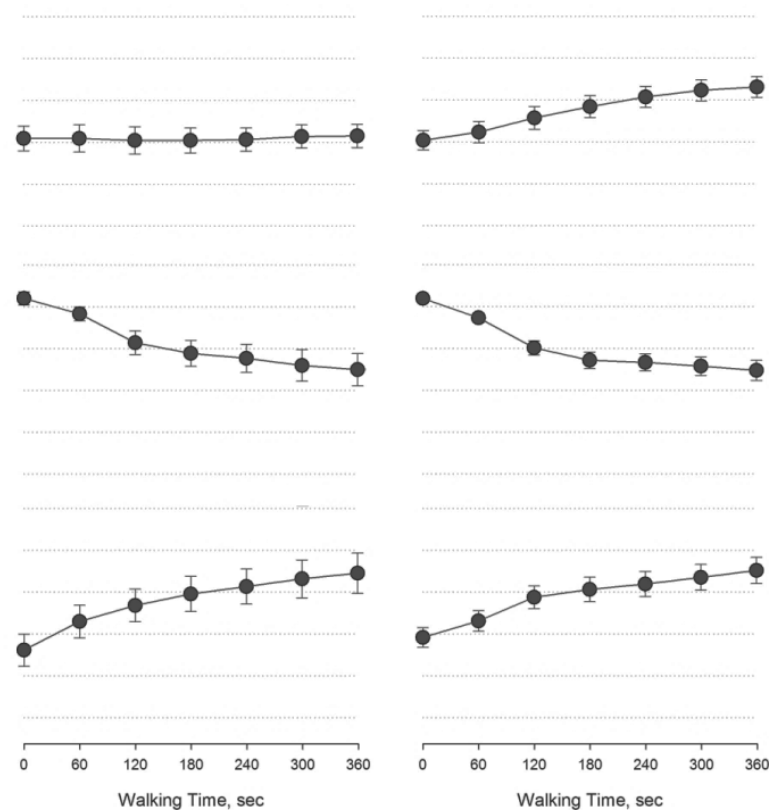

Figure 1. Changes in the variables of $\mathrm{T}_{\mathrm{C}} \mathrm{PCO}_{2}, \mathrm{SpO}_{2}$ and Heart Rate in each minute (means/min) of the 6MWT according to the $\mathrm{CO}_{2}$ retention

The changes of $\mathrm{SpO}_{2}$ and Heart Rate in each minute of the 6MWT were similar for all of the three patterns of $\mathrm{T}_{\mathrm{C}} \mathrm{PCO}_{2}$ response to exercise. Error bars represent the Standard Error of Mean (SEM).

\section{DISCUSSION}

This study provides four major observations: (1) $\mathrm{PCO}_{2}$ response to exercise is highly heterogeneous in patients with advanced COPD; (2) half of the patients (50\%) demonstrated $\mathrm{CO}_{2}$ retention, whereas a $31 \%$ of total population exhibited EIH during 
the 6MWT; (3) exercise-induced $\mathrm{CO}_{2}$ retention is mostly determined by worse airflow limitation while EIH is mostly associated with higher baseline $\mathrm{P}_{\mathrm{C}} \mathrm{CO}_{2}$ levels; and (4) overweight BMI may prevent the occurrence of EIH in very severe COPD. To the best of our knowledge, this is the first study to demonstrate the patterns of $\mathrm{T}_{\mathrm{C}} \mathrm{PCO}_{2}$ trends in the 6MWT using continuous recording of $\mathrm{CO}_{2}$ and to reveal the determinants of $\mathrm{CO}_{2}$ retention and EIH during walking in GOLD stage IV.

The physiological basis of $\mathrm{CO}_{2}$ retention and $\mathrm{EIH}$ is multifactorial as it reflects mechanical constraints on ventilation resulting in ventilatory abnormalities ${ }^{16}$, reduced respiratory drive ${ }^{30}$ and impaired physiological characteristics ${ }^{18,31}$ such as increased resting $\mathrm{PCO}_{2}$ levels and indices of airflow limitation. It is also well-documented that physiological dead space is increased in patients with advanced COPD due to underlying $\mathrm{V} / \mathrm{Q}$ mismatch, which requires a higher ventilation to keep alveolar ventilation (and hence $\mathrm{PCO}_{2}$ ) constant. ${ }^{32,}{ }^{33}$ At increased respiratory rates usually during exercise, an inversely proportional decrease in expiratory time often occurs in patients with COPD contributing to the development of dynamic hyperinflation. ${ }^{33}$ Montes de Oca and Celli indicated that EIH is related to lungs' inability to increase ventilation because of factors such as the presence of dynamic hyperinflation. ${ }^{34} \mathrm{O}^{\prime}$ Donnell and colleagues have also reported that the tendency to develop $\mathrm{CO}_{2}$ retention during exercise could be predicted by the degree of dynamic hyperinflation. ${ }^{16}$

In the current study, dynamic hyperinflation may contribute to $\mathrm{CO}_{2}$ retention and $\mathrm{EIH}$. Although there were no differences in static hyperinflation (RV/TLC) between $\mathrm{CO}_{2}$ retainers and non-retainers, a higher airflow limitation may result in greater $\mathrm{CO}_{2}$ retention and $\mathrm{EIH}$ potentially due to higher degree of dynamic hyperinflation at exertion. After categorization based on $\mathrm{CO}_{2}$ retention, the patients of current study tended to have worse airflow limitation compared to non-retainers and this is more distinct and reaches significance when patients are stratified by EIH (Table 3). Indeed, impaired $\mathrm{FEV}_{1}$ can result in air-trapping causing hyperinflation which, in turns, leads to $\mathrm{CO}_{2}$ retention. ${ }^{35}$ However, a number of patients with severely impaired $\mathrm{FEV}_{1}$ did not reached the hypercapnic levels $\left(\mathrm{T}_{\mathrm{C}} \mathrm{PCO}_{2}>45 \mathrm{mmHg}\right)$ during 6MWT. Other factors such as respiratory muscle strength could potentially compensate the negative impact of low $\mathrm{FEV}_{1}$ for the occurrence of dynamic hyperinflation preventing EIH as it has been also reported in previous studies. ${ }^{36-38}$

In this study, resting tidal volume and ventilation has been shown to be comparable between $\mathrm{CO}_{2}$ retainers and non-retainers; however, patients with EIH had more shallow breaths and an overall lower ventilation compared to non-EIH (Table 3). It has been postulated that $\mathrm{CO}_{2}$ retention during exercise is attributed to a "behavioral" adoption of shallow breathing pattern, which serves to minimize intrathoracic pressure perturbations, reduce respiratory discomfort, and mitigate the development of respiratory muscle fatigue ("wise fighter" hypothesis). ${ }^{39}$ Sorli et al. demonstrated that increase in $\mathrm{PCO}_{2}$ levels was associated with a decreased tidal volume and reduced 
alveolar ventilation. ${ }^{40}$ Our findings suggested that shallow breathing and low ventilation are characteristics of patients with $\mathrm{EIH}$ rather than those who retain $\mathrm{CO}_{2}$ but do not reach the hypercapnic levels during the 6MWT (Table 3). Also, health and mood status (CRQ / HADS scores) did not correlate with $\mathrm{T}_{C} \mathrm{PCO}_{2}$ (Pearson's $r=0.12$ to $0.19, p>0.17$ ) during the 6MWT, which may imply that several factors can impact on health and mood status besides the lung function impairment as represented by $\mathrm{T}_{\mathrm{C}} \mathrm{PCO}_{2}$. This again illustrates the complex intertwinement of what we measure, expect and the symptoms patients suffer of.

Interestingly, clear differences were found in BMI of EIH and non-EIH patients (Table 3). Although it is well established that obesity causes decreases in resting lung volumes $^{41}$, the ventilatory responsiveness to hypercapnia can be higher in patients with increased weight compared those with weight loss. ${ }^{42,43}$ Indeed, chemoreflex responses to hypercapnia have been shown to be potentiated in eucapnic obese subjects. ${ }^{44}$ Moreover, obesity, compared to normal range $\mathrm{BMI}$, is associated to lower resting and dynamic hyperinflation, lower mechanical loading and thus can result in lower $\mathrm{CO}_{2}$ retention. ${ }^{45-48}$ Evidence also has shown that COPD patients with low BMI had 20 times higher risk of having $\mathrm{CO}_{2}$ retention during an acute exacerbation than those with normal $\mathrm{BMI}^{49}$ The findings of the current study indicate that overweight BMI reduces the risk of $\mathrm{EIH}$ in very severe COPD (Tables 3 and 5), which can be attributed to a potential higher ventilatory responsiveness, less dynamic hyperinflation and lower mechanical loading during the 6MWT.

According to the findings, $\mathrm{EIH}$ is highly dependent on $\mathrm{PCO}_{2}$ baseline values (Table 3 and 4). When EIH is defined as reaching above a certain threshold, this is very much defined by the baseline levels. Indeed, patients who exhibited EIH had increased baseline- $\mathrm{P}_{\mathrm{C}} \mathrm{CO}_{2}$ and pre-walk $\mathrm{T}_{\mathrm{C}} \mathrm{PCO}_{2}$ levels compared to non-ElH. However, high baseline $\mathrm{PCO}_{2}$ levels are not always predictive of the increase in $\mathrm{PCO}_{2}$ and $\mathrm{ElH}^{50}$ A minority of patients (23\%) who did not exhibit ElH presented high baseline $\mathrm{T}_{\mathrm{C}} \mathrm{PCO}_{2}$ levels $\left(\mathrm{T}_{\mathrm{C}} \mathrm{PCO}_{2} \geq 42 \mathrm{mmHg}\right.$ ). Furthermore, increased resting $\mathrm{P}_{\mathrm{C}} \mathrm{CO}_{2}$ levels seem to modify dyspnea sensation at rest. Nevertheless, post-walk dyspnea was comparable in both groups stratified on the presence of $\mathrm{EIH}$ as well as the exercise capacity and $\mathrm{SpO}_{2}$ nadir (Table 4).

A logistical regression model for the prediction of $\mathrm{CO}_{2}$ retention and $\mathrm{EIH}$ (adjusted for age, sex, and LTOT) showed that $\mathrm{FEV}_{1}$ and pre-walk dyspnea determine $\mathrm{CO}_{2}$ retention, whereas baseline- $\mathrm{P}_{\mathrm{C}} \mathrm{CO}_{2}$ levels and pre-walk dyspnea had a strong influence on the prevalence of EIH in patients with very severe COPD (Table 5). These findings may imply that airflow limitation is more important factor for $\mathrm{CO}_{2}$ retention; however, baseline$\mathrm{P}_{\mathrm{C}} \mathrm{CO}_{2}$ levels are more crucial for reaching hypercapnic $\mathrm{CO}_{2}$ values during the 6MWT. In addition, a higher BMI reduces the risk of EIH in patients with very severe COPD (Table 5). 


\section{Strengths and weaknesses}

There are certain limitations to our study. The SenTec electrode has been shown to be accurate enough under resting conditions and also transcutaneous $\mathrm{PCO}_{2}$-measurements have been already validated at CPET (TOSCA 500 monitor). ${ }^{9}$ Then again, the accuracy of SenTec recordings has not been proved yet during the 6MWT. Pilot evaluation $(n=10$ patients with COPD) for the agreement of $\mathrm{PCO}_{2}$ recordings between capillary $\mathrm{BGA}$ and SenTec device, however, showed a very strong intraclass correlation coefficient (ICC) absolute agreement for pre-walk (ICC $=0.98,95 \% \mathrm{Cl}: 0.93-0.99)$ and post-walk (ICC = 0.98, 95\% Cl: 0.73-0.99) values of the 6MWT (Supplementary table S5 and figure S4). Another limitation of the study is that respiratory measurements such as ventilation, tidal flow-volume loops, end-tidal $\mathrm{PCO}_{2}$ and the detection of dynamic hyperinflation were not performed during walking. There was no detailed assessment of dynamic lung mechanics, which would have provided us with important information for patients' respiratory profile during the 6MWT. Also, a power analysis was not performed and our sample size may be relatively small for this kind of analysis and also included only very severe COPD patients. Despite these limitations, this study offers a novel analysis of $\mathrm{PCO}_{2}$ response to exercise and the determinants of $\mathrm{CO}_{2}$ retention during the 6MWT. The detection and interpretation of $\mathrm{CO}_{2}$ retention and $\mathrm{EIH}$ during exercise could be an enhanced diagnostic tool for the clinical management of COPD. Furthermore, it may be useful information for the design of tailored pulmonary rehabilitation programs for patients with elevated $\mathrm{PCO}_{2}$ levels and $\mathrm{EIH}$. Future studies need to reproduce these findings at isoload exercise time and evaluate the impact of $\mathrm{PCO}_{2}$ retention and $\mathrm{EIH}$ during the 6MWT in respect to prognosis and respiratory failure.

\section{CONCLUSIONS}

The response of $\mathrm{PCO}_{2}$ to $6 \mathrm{MWT}$ is highly heterogeneous in patients with very severe COPD. However, $\mathrm{CO}_{2}$ retention is related to worse airflow limitation and pre-walk dyspnea, whereas the occurrence of $\mathrm{EIH}$ is associated to higher baseline $\mathrm{P}_{\mathrm{C}} \mathrm{CO}_{2}$ levels and pre-walk dyspnea. Reduced $\mathrm{FEV}_{1}$ and elevated baseline- $\mathrm{P}_{\mathrm{C}} \mathrm{CO}_{2}$ together with prewalk dyspnea increase the risk for $\mathrm{CO}_{2}$-retention and $\mathrm{EIH}$ during the $6 \mathrm{MWT}$. In contrast, overweight BMI seems to carry a protective effect against EIH in very severe COPD. Monitoring of $\mathrm{T}_{\mathrm{C}} \mathrm{PCO}_{2}$ during 6MWT might help to identify patients who need special attention regarding to $\mathrm{EIH}$. 


\section{REFERENCES}

1. Holland AE, Spruit MA, Troosters T, et al. An official European Respiratory Society/American Thoracic Society technical standard: field walking tests in chronic respiratory disease. Eur Respir J. 2014; 44: 142846.

2. Singh SJ, Puhan MA, Andrianopoulos V, et al. An official systematic review of the European Respiratory Society/American Thoracic Society: measurement properties of field walking tests in chronic respiratory disease. Eur Respir J. 2014; 44: 1447-78.

3. Spruit MA, Polkey MI, Celli B, et al. Predicting outcomes from 6-minute walk distance in chronic obstructive pulmonary disease. J Am Med Dir Assoc. 2012; 13: 291-7.

4. Poulain M, Durand F, Palomba B, et al. 6-minute walk testing is more sensitive than maximal incremental cycle testing for detecting oxygen desaturation in patients with COPD. Chest. 2003; 123: 1401-7.

5. Andrianopoulos V, Franssen FM, Peeters JP, et al. Exercise-induced oxygen desaturation in COPD patients without resting hypoxemia. Respir Physiol Neurobiol. 2014; 190: 40-6.

6. Casanova C, Cote C, Marin JM, et al. Distance and oxygen desaturation during the 6-min walk test as predictors of long-term mortality in patients with COPD. Chest. 2008; 134: 746-52.

7. Andrianopoulos V, Wouters EF, Pinto-Plata VM, et al. Prognostic value of variables derived from the sixminute walk test in patients with COPD: Results from the ECLIPSE study. Respir Med. 2015; 109: 1138-46.

8. Domingo C, Canturri E, Lujan M, Moreno A, Espuelas H and Marin A. [Transcutaneous measurement of partial pressure of carbon dioxide and oxygen saturation: validation of the SenTec monitor]. Arch Bronconeumol. 2006; 42: 246-51.

9. Stege G, van den Elshout FJ, Heijdra YF, van de Ven MJ, Dekhuijzen PN and Vos PJ. Accuracy of transcutaneous carbon dioxide tension measurements during cardiopulmonary exercise testing. Respiration. 2009; 78: 147-53.

10. Nicolini A and Ferrari MB. Evaluation of a transcutaneous carbon dioxide monitor in patients with acute respiratory failure. Ann Thorac Med. 2011; 6: 217-20.

11. Chhajed PN, Miedinger D, Baty F, et al. Comparison of combined oximetry and cutaneous capnography using a digital sensor with arterial blood gas analysis. Scand J Clin Lab Invest. 2010; 70: 60-4.

12. Oliven A, Kelsen SG, Deal EC and Cherniack NS. Mechanisms underlying CO2 retention during flowresistive loading in patients with chronic obstructive pulmonary disease. J Clin Invest. 1983; 71: 1442-9.

13. Sieker $\mathrm{HO}$ and Hickam JB. Carbon dioxide intoxication: the clinical syndrome, its etiology and management with particular reference to the use of mechanical respirators. Medicine. 1956; 35: 389423.

14. Gorini M, Misuri G, Corrado A, et al. Breathing pattern and carbon dioxide retention in severe chronic obstructive pulmonary disease. Thorax. 1996; 51: 677-83.

15. Light $\mathrm{R}$, Mahutte $\mathrm{C}$ and Brown S. Etiology of carbon dioxide retention at rest and during exercise in chronic airflow obstruction. Chest. 1988; 94: 61-7.

16. O'Donnell DE, D'Arsigny C, Fitzpatrick $M$ and Webb KA. Exercise hypercapnia in advanced chronic obstructive pulmonary disease: the role of lung hyperinflation. Am J Respir Crit Care Med. 2002; 166: 663-8.

17. Rodriguez-Roisin R, Drakulovic M, Rodriguez DA, Roca J, Barbera JA and Wagner PD. Ventilationperfusion imbalance and chronic obstructive pulmonary disease staging severity. J Appl Physiol (1985). 2009; 106: 1902-8.

18. Simard AA, Maltais $F$ and LeBlanc $P$. Functional outcome of patients with chronic obstructive pulmonary disease and exercise hypercapnia. Eur Respir J. 1995; 8: 1339-44.

19. Laserna E, Sibila O, Aguilar PR, et al. Hypocapnia and hypercapnia are predictors for ICU admission and mortality in hospitalized patients with community-acquired pneumonia. Chest. 2012; 142: 1193-9.

20. Lindinger MI and Heigenhauser GJ. Effects of gas exchange on acid-base balance. Compr Physiol. 2012; 2: 2203-54. 


\section{Chapter 7}

21. Ahmadi Z, Bornefalk-Hermansson A, Franklin KA, Midgren B and Ekstrom MP. Hypo- and hypercapnia predict mortality in oxygen-dependent chronic obstructive pulmonary disease: a population-based prospective study. Respir Res. 2014; 15: 30.

22. Budweiser S, Jorres RA and Pfeifer M. Treatment of respiratory failure in COPD. Int J Chron Obstruct Pulmon Dis. 2008; 3: 605-18.

23. Prior JG, Powlson M, Cochrane GM and Wolff CB. Ventilatory changes during exercise and arterial PCO2 oscillations in chronic airway obstruction patients. J Appl Physiol (1985). 1985; 58: 1942-8.

24. Liu Z, Vargas F, Stansbury D, Sasse SA and Light RW. Comparison of the end-tidal arterial PCO2 gradient during exercise in normal subjects and in patients with severe COPD. Chest. 1995; 107: 1218-24.

25. Diaz O, Morales A, Osses R, Klaassen J, Lisboa C and Saldias F. [Six-minute-walk test and maximum exercise test in cycloergometer in chronic obstructive pulmonary disease. Are the physiological demands equivalent?]. Arch Bronconeumol. 2010; 46: 294-301.

26. Herrmann C. International experiences with the Hospital Anxiety and Depression Scale--a review of validation data and clinical results. J Psychosom Res. 1997; 42: 17-41.

27. Guyatt GH, Berman LB, Townsend M, Pugsley SO and Chambers LW. A measure of quality of life for clinical trials in chronic lung disease. Thorax. 1987; 42: 773-8.

28. Rodriguez P, Lellouche F, Aboab J, Buisson CB and Brochard L. Transcutaneous arterial carbon dioxide pressure monitoring in critically ill adult patients. Intensive Care Med. 2006; 32: 309-12.

29. DeLong ER, DeLong DM and Clarke-Pearson DL. Comparing the areas under two or more correlated receiver operating characteristic curves: a nonparametric approach. Biometrics. 1988; 44: 837-45.

30. Markou NK, Myrianthefs PM and Baltopoulos GJ. Respiratory failure: an overview. Critical care nursing quarterly. 2004; 27: 353-79.

31. Sciurba FC, Rogers RM, Keenan RJ, et al. Improvement in pulmonary function and elastic recoil after lung-reduction surgery for diffuse emphysema. N Engl J Med. 1996; 334: 1095-9.

32. O'Donnell DE and Parker CM. COPD exacerbations. 3: Pathophysiology. Thorax. 2006; 61: 354-61.

33. Puente-Maestu $L$ and Stringer WW. Hyperinflation and its management in COPD. Int J Chron Obstruct Pulmon Dis. 2006; 1: 381-400.

34. Montes de Oca M and Celli BR. Respiratory muscle recruitment and exercise performance in eucapnic and hypercapnic severe chronic obstructive pulmonary disease. Am J Respir Crit Care Med. 2000; 161: 880-5.

35. Pellegrino R and Brusasco V. Lung hyperinflation and flow limitation in chronic airway obstruction. Eur Respir J. 1997; 10: 543-9.

36. De Vito EL. [Causes of $\mathrm{CO} 2$ retention in patients with chronic obstructive lung disease]. Medicina (B Aires). 1993; 53: 350-6.

37. Begin P and Grassino A. Inspiratory muscle dysfunction and chronic hypercapnia in chronic obstructive pulmonary disease. Am Rev Respir Dis. 1991; 143: 905-12.

38. Kortianou EA, Aliverti A, Louvaris Z, et al. Limitation in tidal volume expansion partially determines the intensity of physical activity in COPD. J Appl Physiol (1985). 2015; 118: 107-14.

39. Younes M. Determinants of thoracic excursion during exercise. New York: Marcel Dekker, 1991.

40. Sorli J, Grassino A, Lorange G and Milic-Emili J. Control of breathing in patients with chronic obstructive lung disease. Clin Sci Mol Med. 1978; 54: 295-304.

41. Jones RL and Nzekwu MM. The effects of body mass index on lung volumes. Chest. 2006; 130: 827-33.

42. Chapman KR, Himal HS and Rebuck AS. Ventilatory responses to hypercapnia and hypoxia in patients with eucapnic morbid obesity before and after weight loss. Clin Sci (Lond). 1990; 78: 541-5.

43. Emirgil $\mathrm{C}$ and Sobol BJ. The effects of weight reduction on pulmonary function and the sensitivity of the respiratory center in obesity. Am Rev Respir Dis. 1973; 108: 831-42.

44. Narkiewicz K, Kato M, Pesek CA and Somers VK. Human obesity is characterized by a selective potentiation of central chemoreflex sensitivity. Hypertension. 1999; 33: 1153-8.

45. Laviolette L, Sava F, O'Donnell DE, et al. Effect of obesity on constant workrate exercise in hyperinflated men with COPD. BMC Pulm Med. 2010; 10: 33. 
46. Ora J, Laveneziana P, Ofir D, Deesomchok A, Webb KA and O'Donnell DE. Combined effects of obesity and chronic obstructive pulmonary disease on dyspnea and exercise tolerance. Am J Respir Crit Care Med. 2009; 180: 964-71.

47. Franssen FM, O'Donnell DE, Goossens GH, Blaak EE and Schols AM. Obesity and the lung: 5. Obesity and COPD. Thorax. 2008; 63: 1110-7.

48. O'Donnell DE, Deesomchok A, Lam YM, et al. Effects of BMI on static lung volumes in patients with airway obstruction. Chest. 2011; 140: 461-8.

49. Vishnu SM, Anupama N, Harsha DS, et al. Low body mass index and low serum albumin level as risk factors for carbon dioxide retention during acute exacerbation of chronic obstructive pulmonary disease. Int. j. innov. res. sci. eng. 2013; 2: 3508.

50. Dempsey JA. Exercise carbon dioxide retention in chronic obstructive pulmonary disease: a case for ventilation/perfusion mismatch combined with hyperinflation. Am J Respir Crit Care Med. 2002; 166: 634-5.

51. Quanjer PH, Tammeling GJ, Cotes JE, Pedersen OF, Peslin R and Yernault JC. Lung volumes and forced ventilatory flows. Eur Respir J. 1993; 6 Suppl 16: 5-40.

52. Troosters T, Gosselink R and Decramer M. Six minute walking distance in healthy elderly subjects. Eur Respir J. 1999; 14: 270-4. 


\section{SUPPLEMENTAL MATERIAL}

Table S1. Borg's modified scale for rating dyspnea and/or leg fatigue

slight (2)

\section{very slight (1)}

\section{Nothing at all (0)}

The modified "Borg-scale" for rating dyspnea and/or leg fatigue, as used at Schoen Klinik Berchtesgadener Land* in Germany (*here it is presented translated from German to English and in grayscale).

Table S2. Pearson's correlations between $\mathrm{T}_{\mathrm{C}} \mathrm{PCO}_{2}$ response to 6MWT and patients' characteristics

\begin{tabular}{|c|c|c|c|c|c|c|c|c|c|}
\hline & $\begin{array}{l}\mathrm{PcO}_{2} \\
\text { rest }\end{array}$ & $\begin{array}{l}\mathrm{PcCO}_{2} \\
\text { rest }\end{array}$ & $\begin{array}{l}\mathrm{FEV}_{1} \\
\% \text { pred }\end{array}$ & $\begin{array}{l}\text { DLCO } \\
\text { \%pred }\end{array}$ & $\begin{array}{l}\text { Pi max } \\
\text { \%pred }\end{array}$ & $\begin{array}{l}\text { 6MWD } \\
\text { \%pred }\end{array}$ & $\begin{array}{l}\mathrm{SpO}_{2} \\
\text { nadir }\end{array}$ & $\begin{array}{l}\text { Dyspnea } \\
\text { pre }\end{array}$ & $\begin{array}{l}\text { Dyspnea } \\
\text { post }\end{array}$ \\
\hline $\mathrm{T}_{\mathrm{C}} \mathrm{PCO}_{2}$, pre-walk & 0.01 & $0.56 * *$ & -0.13 & -0.10 & $-0.27^{*}$ & -0.22 & 0.05 & 0.03 & -0.01 \\
\hline $\begin{array}{l}\mathrm{T}_{\mathrm{C}} \mathrm{PCO}_{2} \text {, post-walk } \\
\text { (peak values) }\end{array}$ & 0.13 & $0.53^{* *}$ & $-0.32 *$ & -0.08 & -0.20 & -0.11 & -0.09 & 0.23 & 0.20 \\
\hline $\begin{array}{l}\mathrm{T}_{\mathrm{C}} \mathrm{PCO}_{2}[\Delta] \\
\text { (nadir-peak) }\end{array}$ & 0.13 & 0.13 & $-0.26 *$ & -0.03 & 0.08 & 0.12 & 0.17 & 0.20 & 0.24 \\
\hline
\end{tabular}

Abbreviations: $\mathrm{T}_{\mathrm{C}} \mathrm{PCO}_{2}$ : Transcutaneous carbon-dioxide partial-pressure; $\mathrm{FEV}_{1}$ : forced expiratory volume in 1 sec; DLCO: carbon monoxide diffusing capacity; Pi max: Maximal inspiratory mouth pressure; $\mathrm{SpO}_{2}$ : Saturation of peripheral oxygen; 6MWD: Six-minute walk distance. 
Table S3. Cut-off points and the ROC curve parameters for the prediction of $\mathrm{CO}_{2}$ retention

\begin{tabular}{|c|c|c|c|c|c|c|c|c|}
\hline \multirow[b]{2}{*}{$\begin{array}{l}\text { Predictive } \\
\text { index }\end{array}$} & \multirow[b]{2}{*}{$\begin{array}{l}\text { Best } \\
\text { cut-off }\end{array}$} & \multicolumn{7}{|c|}{ ROC curve parameters } \\
\hline & & $\begin{array}{l}\text { AUC } \\
\text { segment }\end{array}$ & $\begin{array}{l}\text { Sensitivity } \\
(\%)\end{array}$ & $\begin{array}{l}95 \% \mathrm{Cl} \\
\text { Binomial } \\
\text { exact }\end{array}$ & $\begin{array}{l}\text { Specificity } \\
(\%)\end{array}$ & $\begin{array}{l}95 \% \mathrm{Cl} \\
\text { Binomial } \\
\text { exact }\end{array}$ & $\begin{array}{l}\text { Likelif } \\
\text { LR+ }\end{array}$ & $\begin{array}{l}\text { ood Ratio } \\
\text { LR- }\end{array}$ \\
\hline Age, years & $\geq 60$ & 0.52 & 0.68 & $0.49-0.83$ & 0.39 & $0.22-0.58$ & 0.90 & 1.20 \\
\hline $\mathrm{BMI}, \mathrm{kg} / \mathrm{m}^{2}$ & $\leq 25$ & 0.61 & 0.74 & $0.55-0.88$ & 0.45 & $0.27-0.64$ & 1.35 & 0.57 \\
\hline $\mathrm{FEV}_{1}, \%$ pred. & $\leq 36$ & 0.60 & 0.81 & $0.63-0.93$ & 0.48 & $0.30-0.67$ & 1.56 & 0.40 \\
\hline $\mathrm{P}_{\mathrm{C}} \mathrm{O}_{2}, \mathrm{mmHg}$ & $\leq 63$ & 0.54 & 0.67 & $0.49-0.83$ & 0.29 & $0.14-0.48$ & 1.05 & 0.90 \\
\hline $\mathrm{P}_{\mathrm{C}} \mathrm{CO}_{2}, \mathrm{mmHg}$ & $\geq 41$ & 0.53 & 0.42 & $0.25-0.61$ & 0.65 & $0.45-0.81$ & 1.18 & 0.90 \\
\hline Pi max, \%pred. & $\leq 64$ & 0.50 & 0.64 & $0.44-0.81$ & 0.53 & $0.34-0.72$ & 1.38 & 0.67 \\
\hline Dyspnea pre-walk & $\geq 2$ & 0.58 & 0.58 & $0.39-0.76$ & 0.67 & $0.47-0.83$ & 1.74 & 0.63 \\
\hline
\end{tabular}

Abbreviations: $\mathrm{BMI}$ : Body mass index; $\mathrm{FEV}_{1}$ : forced expiratory volume in $1 \mathrm{sec} ; \mathrm{P}_{\mathrm{C}} \mathrm{O}_{2}$ : Capillary oxygen partial pressure; $\mathrm{P}_{\mathrm{C}_{2}}$ : Capillary carbon-dioxide partial pressure; Pi max: Maximal inspiratory mouth pressure; AUC: Area under the curve.

Table S4. Cut-off points and the ROC curve parameters for the prediction of $\mathrm{CO}_{2}$ retention

\begin{tabular}{|c|c|c|c|c|c|c|c|c|}
\hline \multirow[b]{2}{*}{$\begin{array}{l}\text { Predictive } \\
\text { index }\end{array}$} & \multirow[b]{2}{*}{$\begin{array}{l}\text { Best } \\
\text { cut-off }\end{array}$} & \multicolumn{7}{|c|}{ ROC curve parameters } \\
\hline & & $\begin{array}{l}\text { AUC } \\
\text { segment }\end{array}$ & $\begin{array}{l}\text { Sensitivity } \\
(\%)\end{array}$ & $\begin{array}{l}95 \% \mathrm{Cl} \\
\text { Binomial } \\
\text { exact }\end{array}$ & $\begin{array}{l}\text { Specificity } \\
(\%)\end{array}$ & $\begin{array}{l}95 \% \mathrm{Cl} \\
\text { Binomial } \\
\text { exact }\end{array}$ & $\begin{array}{l}\text { Likeli } \\
\text { LR+ }\end{array}$ & $\begin{array}{l}\text { ood Ratio } \\
\text { LR- }\end{array}$ \\
\hline Age, years & $\geq 60$ & 0.52 & 0.63 & $0.38-0.84$ & 0.35 & $0.21-0.51$ & 0.97 & 1.06 \\
\hline BMI, $\mathrm{kg} / \mathrm{m}^{2}$ & $\leq 25$ & 0.68 & 0.84 & $0.60-0.97$ & 0.44 & $0.29-0.60$ & 1.51 & 0.36 \\
\hline $\mathrm{FEV}_{1}, \%$ pred. & $\leq 36$ & 0.64 & 0.84 & $0.60-0.97$ & 0.42 & $0.27-0.58$ & 1.45 & 0.38 \\
\hline $\mathrm{P}_{\mathrm{C}} \mathrm{O}_{2}, \mathrm{mmHg}$ & $\leq 63$ & 0.54 & 0.84 & $0.60-0.97$ & 0.37 & $0.23-0.53$ & 1.34 & 0.42 \\
\hline $\mathrm{P}_{\mathrm{C}} \mathrm{CO}_{2}, \mathrm{mmHg}$ & $\geq 41$ & 0.71 & 0.63 & $0.38-0.84$ & 0.67 & $0.52-0.81$ & 1.94 & 0.55 \\
\hline Pi max, \%pred. & $\leq 60$ & 0.68 & 0.76 & $0.50-0.93$ & 0.61 & $0.45-0.76$ & 1.96 & 0.39 \\
\hline Dyspnea pre-walk & $\geq 3$ & 0.66 & 0.53 & $0.30-0.76$ & 0.76 & $0.61-0.88$ & 2.21 & 0.62 \\
\hline
\end{tabular}

Abbreviations: $\mathrm{BMI}$ : Body mass index; $\mathrm{FEV}_{1}$ : forced expiratory volume in $1 \mathrm{sec} ; \mathrm{P}_{\mathrm{C}} \mathrm{O}_{2}$ : Capillary oxygen partial pressure; $\mathrm{P}_{\mathrm{C}} \mathrm{CO}_{2}$ : Capillary carbon-dioxide partial pressure; Pi max: Maximal inspiratory mouth pressure; $A \cup C$ : Area under the curve.

Table S5. Agreement between CBG analysis and SenTec recordings of $\mathrm{PCO}_{2}$ during the 6MWT

\begin{tabular}{|c|c|c|c|c|c|c|c|c|c|c|}
\hline \multirow[b]{2}{*}{ ID } & \multirow[b]{2}{*}{ Sex } & \multirow[b]{2}{*}{ Age } & \multirow{2}{*}{$\begin{array}{l}\text { GOLD } \\
\text { Stage }\end{array}$} & \multicolumn{3}{|c|}{$\mathrm{PCO}_{2}$ pre-walk, $\mathrm{mmHg}$} & \multicolumn{3}{|c|}{$\mathrm{PCO}_{2}$ post-walk, $\mathrm{mmHg}$} & \multirow[b]{2}{*}{$\mathrm{O}_{2}$ Liters } \\
\hline & & & & $C B G$ & SenTec & Difference & $C B G$ & SenTec & Difference & \\
\hline 1 & M & 55 & 4 & 60.2 & 60.1 & -0.1 & 63.5 & 60.8 & -2.7 & 2.0 \\
\hline 2 & M & 62 & 4 & 40.0 & 39.0 & -1.0 & 40.3 & 39.1 & -1.2 & 3.0 \\
\hline 3 & M & 84 & 2 & 33.0 & 32.2 & -0.8 & 31.7 & 30.1 & -1.6 & - \\
\hline 4 & M & 74 & 3 & 30.0 & 30.9 & +0.9 & 30.6 & 30.4 & -0.2 & - \\
\hline 5 & M & 64 & 4 & 49.5 & 51.2 & +1.7 & 47.6 & 44.8 & -2.8 & 3.0 \\
\hline 6 & $\mathrm{~F}$ & 59 & 4 & 38.9 & 36.6 & -2.3 & 43.8 & 43.1 & -0.7 & 2.0 \\
\hline 7 & $\mathrm{~F}$ & 44 & 4 & 32.9 & 33.9 & +1.0 & 33.1 & 33.5 & +0.4 & - \\
\hline 8 & $\mathrm{~F}$ & 69 & 4 & 35.8 & 36.2 & +0.4 & 38.8 & 36.4 & -2.4 & 2.0 \\
\hline 9 & $\mathrm{~F}$ & 63 & 4 & 35.0 & 34.4 & -0.6 & 38.3 & 35.4 & -2.9 & 3.0 \\
\hline 10 & $\mathrm{~F}$ & 71 & 4 & 46.4 & 50.9 & +4.5 & 53.7 & 53.3 & -0.4 & 2.5 \\
\hline
\end{tabular}

Abbreviations: CBG: Capillary Blood Gas values; SenTec: Sentec monitoring-system measures of transcutaneous carbon-dioxide partial-pressure $\left(\mathrm{T}_{\mathrm{C}} \mathrm{PCO}_{2}\right)$. 
A. 1socapnic pattern $(n=16,26 \%)$

$\mathrm{CO}_{2}$ Non-Retainers $(\mathrm{n}=31)$


Start 60120180240300360 Start 60120180240300360 Start $60 \quad 120180240300360$ Start 60120180240300360 Walking time, sec Walking time, sec

Walking time, sec

Walking time, sec

\section{B. Decreasing pattern $(n=15,24 \%)$}
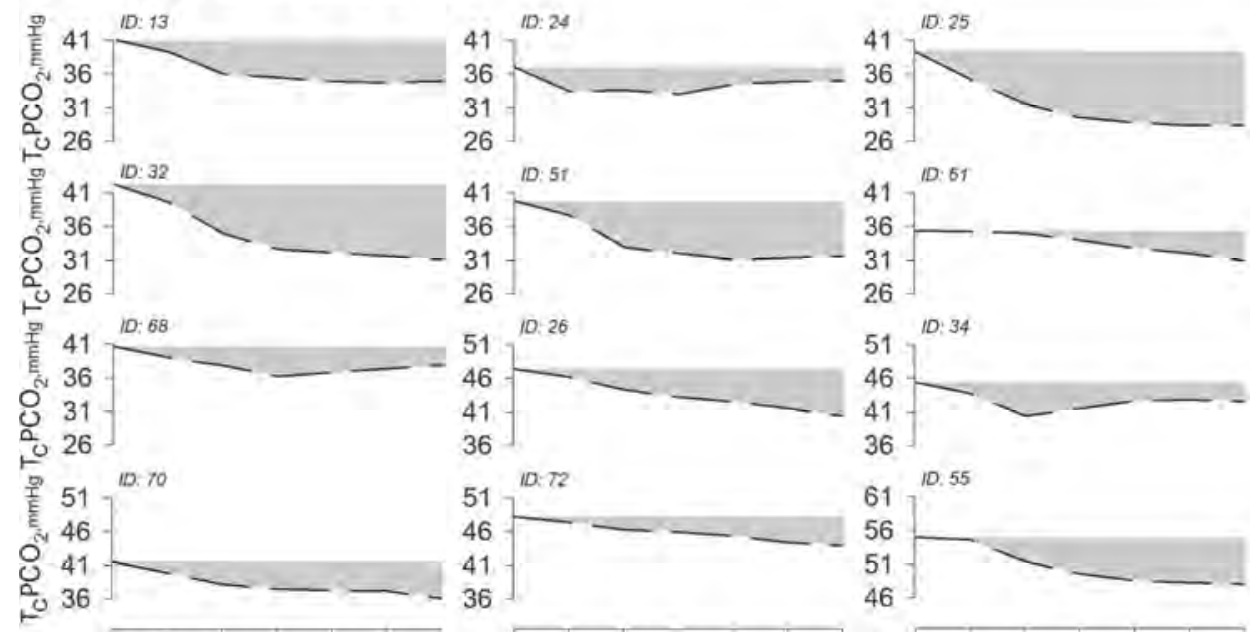

26
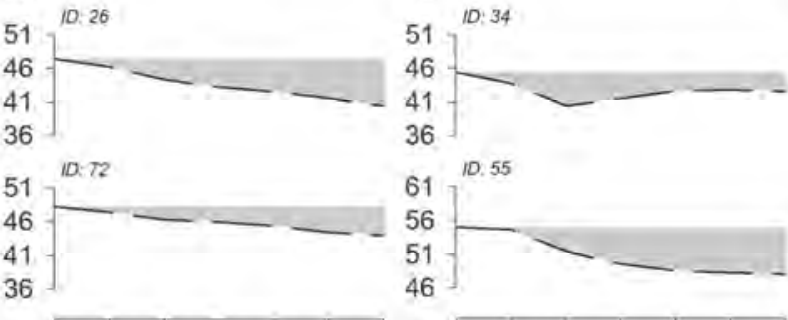

Start 60120180240300360 Start 60120180240300360 Start 6012018024030036 Walking time, sec

Figure S1. Individual changes in $\mathrm{T}_{\mathrm{C}} \mathrm{PCO}_{2}$ during the 6MWT of $\mathrm{CO}_{2}$ "non-retainers"

Individual changes in $\mathrm{T}_{\mathrm{C}} \mathrm{PCO}_{2}$ were observed during the $6 \mathrm{MWT}$ of $\mathrm{CO}_{2}$ "non-retainers" including patients with exercise-induced isocapnic or decreasing $\mathrm{T}_{\mathrm{C}} \mathrm{PCO}_{2}$ pattern. 


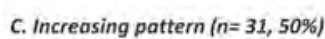

tepCO 2 Retainers $(n=31)$
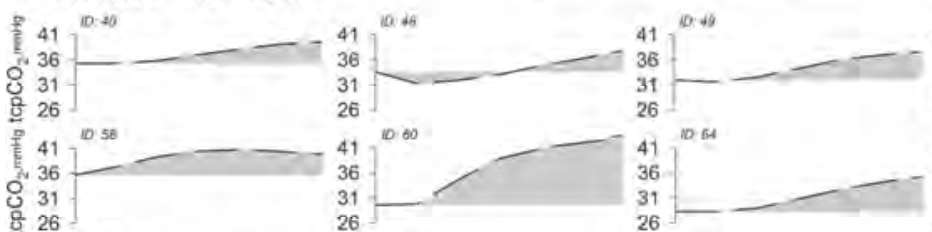

을 26
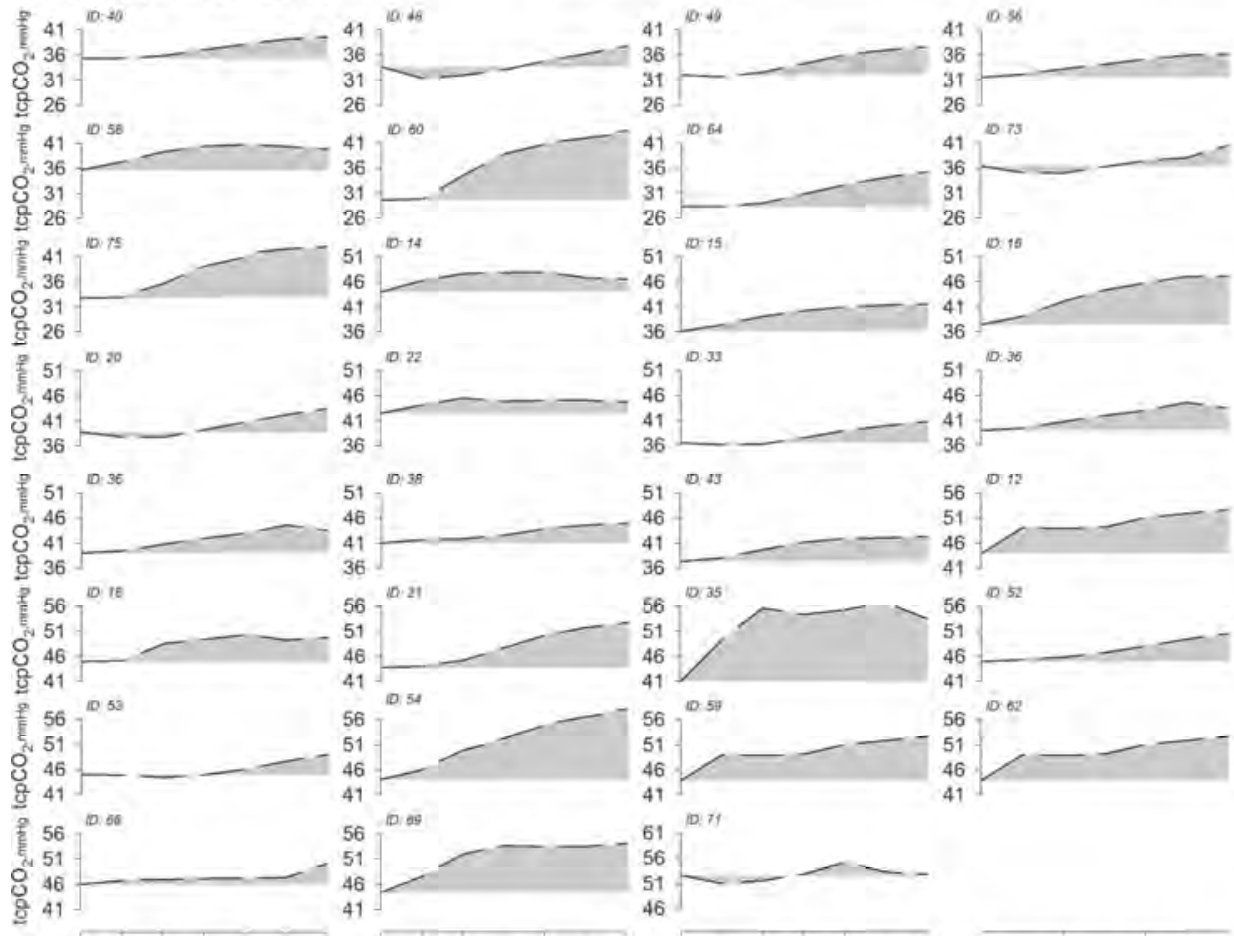

$41 \mathrm{~J}$
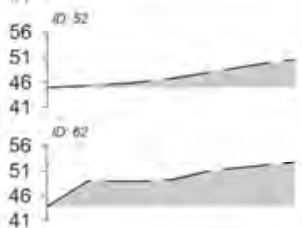

Start 60120180240300360 Start $60 \quad 120180240300360$ Start $60 \quad 120180240300360 \quad$ Start $60 \quad 12018024030036 \mathrm{C}$ Walking time, sec

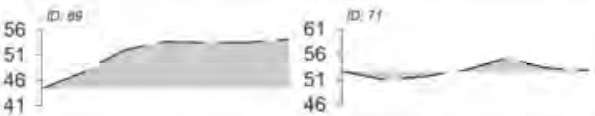

Walking time, sec

Walking time, sec

Figure S2. Individual changes in $\mathrm{T}_{\mathrm{C}} \mathrm{PCO}_{2}$ during the $6 \mathrm{MWT}$ of $\mathrm{CO}_{2}$ "retainers"

Individual changes in $\mathrm{T}_{\mathrm{C}} \mathrm{PCO}_{2}$ were observed during the $6 \mathrm{MWT}$ of $\mathrm{CO}_{2}$ "retainers" including patients with exercise-induced increasing $\mathrm{T}_{\mathrm{C}} \mathrm{PCO}_{2}$ pattern. 
$\mathrm{T}_{\mathrm{C}} \mathrm{PCO}_{2}$ response to $6 \mathrm{MWT}$

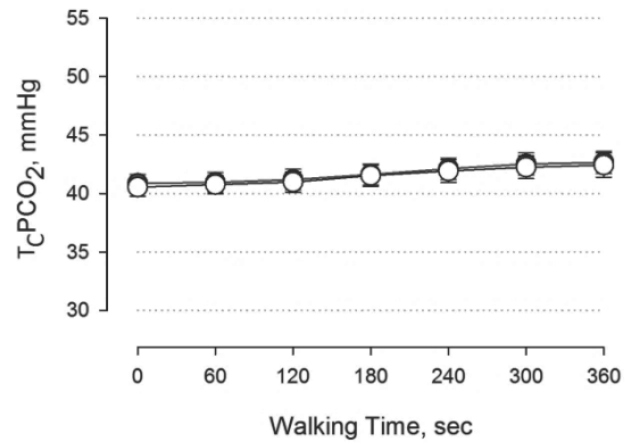

Heart Rate response to 6MWT

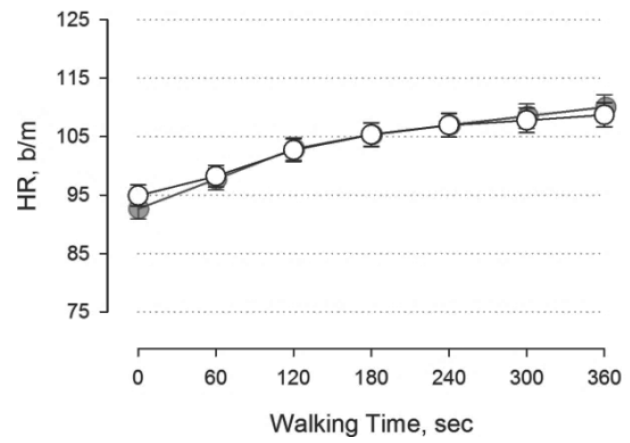

$\mathrm{SpO}_{2}$ response to $6 \mathrm{MWT}$

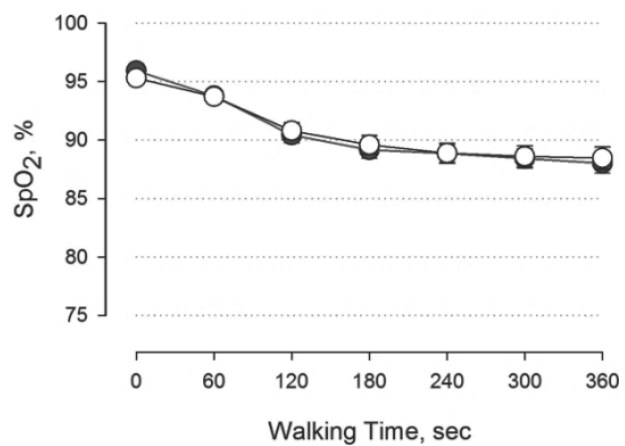

Figure S3. Repeatability of the changes in $\mathrm{T}_{\mathrm{C}} \mathrm{PCO}_{2}, \mathrm{SpO}_{2}$ and Heart Rate in 6MWTs

The repeatability of the changes in $\mathrm{T}_{\mathrm{C}} \mathrm{PCO} 2, \mathrm{SpO}_{2}$ and Heart Rate (mean value/minute) was measured in 6MWTs (longest vs shortest 6MWT). Error bars represent the Standard Error of Mean (SEM).

$\mathrm{PCO}_{2}$

pre-walk values

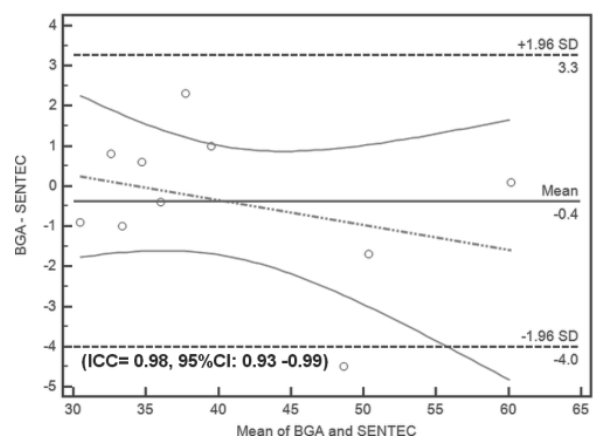

$\mathrm{PCO}_{2}$

post-walk values

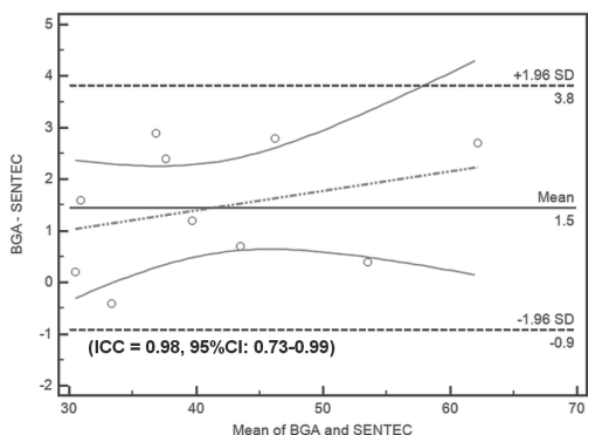

Figure S4. Bland-Altman plots of the means of CBG analysis and SenTec recordings during 6MWT Bland-Altman plots of the means of both methods (CBG analysis and SenTec recordings) during the 6MWT in patients with COPD. Regression lines of differences and 95\% Confidential Intervals are presented. Intraclass Correlation Coefficient (ICC) of absolute agreement and $95 \% \mathrm{Cl}$ were calculated. 
Chapter

General Discussion 

The European Respiratory Society (ERS) and American Thoracic Society (ATS) have recognized the six-minute walk test (6MWT) as a valid and reliable measure of exercise capacity for patients with chronic lung disease. ${ }^{1,2}$ To date, the six-minute walk test (6MWT) is the most frequently employed "self-paced" test of walking capacity in patients with COPD, expressed as the distance walked in six minutes (6MWD). ${ }^{1}{ }^{2}$ The 6MWD is widely-used as clinical outcome measure providing important information about the global and integrated responses of pulmonary and cardiovascular system during exertion, and it has significant prognostic value., 4 From an evidence-based perspective, 6MWD with associated pathophysiological responses such as dyspnea and leg fatigue as well as oxygen desaturation and carbon-dioxide retention at exertion, is of considerable importance in the multidimensional evaluation in COPD regarding intervention effects and prognosis. The 6MWD appears to be responsive to clinical treatments while it also presents a significant association with mortality and hospitalization in patients with COPD. ${ }^{1,2}$ In addition, a number of indices derived from the 6MWT may improve the predictive value of information gained from the 6MWD. However, the interpretation of the 6MWT usually remains restricted to 6MWD as the only parameter used in clinical practice. Other 6MWT-derived variables, such as walking speed (6MWSpeed), six-minute walk-work (6MWW), distance saturation products (DSP), exercise-induced desaturation (EID), and unintended stop(s) during walking can provide healthcare professionals, patients and scientists with important additional and prognostic clinical information. ${ }^{5}$

This thesis aimed to increase the current knowledge for the 6MWT and to strengthen its utility by providing novel insights on the determinants and the interpretation of walking performance as well as demonstrating determinants of exercise-induced oxygen desaturation (EID), $\mathrm{CO}_{2}$ retention and exercise-induce hypercapnia $(E I H)$, and assessing and extending its prognostic value in patients with COPD.

\section{SIX-MINUTE WALK TEST IN COMPARISON WITH CYCLE ENDURANCE TEST (CET)}

In chapter 2 of this thesis, certain characteristics of functional exercise capacity and different factors that determine exercise performance in cycle endurance test (CET) and $6 \mathrm{MWT}$ were investigated. In general, the $6 \mathrm{MWT}$ is characterized by a self-paced walking involving all major muscular groups, whereas CET utilizes primarily the lower body muscles to sustain a constant work-load. ${ }^{6-8}$ The results of chapter 2 demonstrated that the variability of endurance time in CET $\left(C E T-T_{\text {end }}\right)$, even though poorly explained $\left(r^{2}\right.$ : 15.1\%), included the characteristics of age, sex, fat-free mass index (FFMI), GOLD stage, diffusion lung capacity for carbon dioxide (DLCO), dyspnea and leg-fatigue before test. On the other hand, the variance in 6MWD was better explained $\left(r^{2}: 34.0 \%\right)$ by including the 
following characteristics of age, BMI, FFMI, disease severity by GOLD stage, $\mathrm{FEV}_{1}$, $\mathrm{FEV}_{1} / \mathrm{FVC}$, functional residual capacity (FRC), and DLCO, as well as leg-fatigue before test. ${ }^{9}$

Chapter 2 showed that age, FFMI, GOLD stage, DLCO and leg-fatigue are common determinants of CET-T end and the 6MWD. In healthy elderly, aging is related to an important decrease of muscle power ${ }^{10}$ as well as to a less efficient cardiovascular system $^{11}$ and mainly these factors result in lower exercise capacity affecting both cycling and walking performance. ${ }^{12}$ Age-related decrements can also be explained by a general muscle deconditioning as physical activity usually declines with age, while this process in patients with COPD is also being intensified by the progress of disease severity over time. ${ }^{13}$ Another factor which seems to affect both CET and 6MWT in COPD, is the FFMI. In line with the results of chapter 2, a positive correlation has been reported between fat-free mass and the performance in $\mathrm{CET}^{14}$ and $6 \mathrm{MWT} .{ }^{15}$ However, an evaluation of high number of potential confounders such as disease severity, health status, cardiovascular disease, exacerbation rate and oxygen use, might eliminate the relationship between fat-free mass and $6 \mathrm{MWT} .{ }^{16}$ Furthermore, DLCO has been reported as the strongest predictor in exercise intolerance in $\mathrm{COPD}^{17}$ reflecting the high impact of DLCO on cycling and walking performance, while very low DLCO levels are particularly related to the need of oxygen supplementation during exercise. ${ }^{18}$ In agreement with chapter 2, leg-fatigue has been also shown as an important factor limiting exercise in COPD. ${ }^{19}$ The observation of those aforementioned clinical characteristics which are common determinants for CET-T end and 6MWD, suggest that both exercise test can be used to discriminate patients according to underlying disease severity and loss of functional capacity. Indeed, CET and 6MWT seems to be associated with systemic impairment in COPD that is manifested at exertion.

Chapter 2 also demonstrated the different determinants of exercise capacity between CET and the GMWT. Baseline dyspnea and sex were related to CET-Tend, whereas $\mathrm{BMI}$ and lung-function indices of $\mathrm{FEV}_{1}, \mathrm{FEV} \mathrm{V}_{1} / \mathrm{FVC}$, and $\mathrm{FRC}$ were related to $6 M W D$. Previous studies have shown that dyspnea predicts general health status to a greater extent than physiologic measurements in patients with COPD. ${ }^{20,21}$ Therefore, it may be reasonable that $\mathrm{CET}_{-} \mathrm{T}_{\text {end }}$, as determined by rates of dyspnea, mostly reflects an overall impaired health status in COPD rather than lung function impairment which can be better reflected by the 6MWD. ${ }^{22}$ On the other hand, BMI has great impact on legmovement during walking but this is not the case for CET which is a non-weight-bearing test as patient's body weight is supported by the seat. ${ }^{23-25}$ Evidence has also shown that an increase in $\mathrm{FEV}_{1}$ is beneficial for improving walking but not cycling endurance in patients with COPD, which confirms that $\mathrm{FEV}_{1}$ may determine 6MWD but not CET $\mathrm{T}_{\text {end. }}{ }^{26}$ It seems that 6MWD may reflect improvements in lung function independently of the deconditioned state of the peripheral muscles in COPD. Indeed, an increase in 6MWD due to less dynamic hyperinflation, as a result of better lung function, might be better 
represented by the 6MWT which usually causes less quadriceps fatigue compared to CET that imposes a constant work load to the legs. ${ }^{27}$

Interestingly, sex appears to be a strong determinant of CET-T end in contrast to 6MWD. The male population of studied patients achieved a higher peak work rate (WRpeak) and cycling performance at 75\% WR-peak during CET compared to the female group. This trend was also observed by Laviolette and colleagues who demonstrated that women with COPD are disadvantaged during exercise as they reached a critical inspiratory reserve volume earlier, leading to a steep increase in dyspnea and to exercise termination. ${ }^{28}$ In addition, a number of sex-related differences in the clinical expression of COPD have been reported including higher scores on the St. George's Respiratory Questionnaire $(S G R Q)^{29}$ in women that indicates more impaired health status. ${ }^{30}$ Therefore, a general worse experience of health status in women may result in more intensive perceived symptoms limiting the CET- T $_{\text {end }}$. This could be also a reflection of the fact that cycling can evoke more dyspnea because of its unfamiliarity compare to walking which simulates a more routine daily activity.

To the best of our knowledge, chapter 2 was the first report demonstrating the performance quartiles between CET and the 6MWT. The recruitment of different muscle groups between CET and 6MWT and the "external-paced" nature of CET compared to "self-paced" 6MWT, contributes to different ventilatory responses resulting in different levels of perceived dyspnea, leg-fatigue and different physiological responses influencing performance. ${ }^{31}$ The relative weight of several clinical characteristics on CET-T end and 6MWD also varies within performance quartiles of these tests highlighting the complex pathophysiology that influence exercise capacity in COPD. Consequently, clinicians and health professionals should consider the choice of CET or 6MWT as critically important according to the aims of the assessment procedure. Moreover, they need to be aware of patients' clinical characteristics which determine exercise performance in CET and 6MWT, when interpreting or comparing results of both test procedures.

Vivodtzev and colleagues have reported that physiological stress caused by CET is not identical among patients even though cycling is performed at fixed proportion (75\%) of peak incremental workload capacity. ${ }^{32}$ In chapter 2, CET-Tend had a large variation among patients, even though the work load was predefined. In fact, CET at $75 \%$ is influenced by the degree of peripheral muscle deconditioning as the load imposed is fixed and not self-chosen as during the 6MWT. Indeed, this variation may indicate a large heterogeneity in muscle function including different levels of impaired oxidative capacity, muscle atrophy, and physical deconditioning of patients who can additionally present different degrees of exercise-induced desaturation or hypercapnia at exertion. Therefore, it can result in different physiological demands during constant work-load which may lead to different physiological stress during CET that may better reflect the overall state of functional capacity. ${ }^{33-38}$ However, it seems that performing 
CET at $75 \%$ of peak work rate is a feasible level of effort for COPD patients being referred for a pulmonary rehabilitation (PR) program according to the findings of chapter 2.

As suggested before, the 6MWT and CET are not interchangeable; ideally, both tests should be performed in clinical settings for the exercise assessment. The fact that the determinants of CET- $T_{\text {end }}$ cannot be reproducible from a model, which includes a small number of clinical parameters, underscores the importance of CET-Tend as an independent measurement before and after a clinical intervention. In addition, improvements in exercise outcomes after PR program should be evaluated using both $6 \mathrm{MWT}$ and CET as patients response outcomes can differ in 6MWT and CET. ${ }^{39}$ Practical limitations or time and personnel restrictions can impose the use of one test, either $6 \mathrm{MWT}$ or CET. For instance, a 6MWT cannot be performed in daily clinical practice in case of a short hall-way $(<30 \mathrm{~m}$ in length), while CET needs a cardiopulmonary exercise test (CPET) to determine the peak cycling load, which requires equipment and technical support that is not always easily available. Chapter 2 expands the knowledge for the determinants of exercise performance in 6MWT and CET and demonstrates different characteristics of those tests as well as factors that influence cycling and walking emphasizing that in the latter peripheral muscle deconditioning is less of importance.

\section{THE IMPORTANCE OF THE CHOICE OF REFERENCE EQUATION IN 6MWT}

Recent guidelines on the diagnosis and treatment of COPD indicate that assessment of disease severity is more comprehensive by using additional functional criteria such as exercise capacity. ${ }^{40-43}$ The percentage of predicted 6MWD (\%), which represents the exercise capacity in the $6 \mathrm{MWT}$, may be different as calculated by several reference equations which have been formulated under different 6MWT operational settings and for different populations. ${ }^{44-46}$

Chapter 3 indicated that discrepancies among reference values are depending on factors used for the formulation of reference equations including ( $A$ ) demographic and anthropometric characteristics, $(B)$ methodological differences, and $(C)$ health condition of the healthy population. Recommendation for clinicians and health professionals is to choose a 6MWD reference equation which is derived from 6MWTs performed under same operating procedures and derived from same country/region populations. ${ }^{47}$

The 6MWD has shown a considerable variability in several studies. ${ }^{44,48-54}$ Regarding the important influence of demographic and anthropometric characteristics in 6MWD, the variables of age, height, weight, BMI and sex can determine walking performance and explain the $25 \%$ to $66 \%$ of the total variability in 6MWD. ${ }^{44,48-52,54-66}$ More specifically, ageing can contribute to impaired walking performance by muscle loss and weakness with an average loss of muscle strength of $20-40 \%$ in individuals older than 70 
years old resulting in lower gait speed and walking ability. ${ }^{67,68}$ Evidence has also shown that differences in patients' height, which are attributed to genetics, sex and race, are strongly associated to length of stride, which is a component of walking speed, and determines the 6MWD. ${ }^{44}, 51,53,55$ Moreover, differences in patients' weight, as expressed better in $\mathrm{BMI}$, are associated to different energy cost during walking ${ }^{69}$ and may indicate different levels of physical capacity ${ }^{70}$, which determine the 6MWD. For instance, obesity is related to physical inactivity and deconditioning ${ }^{71}$ which result in reduced cardiopulmonary capacity and increased metabolic load for a given amount of exercise with a negative effect on walking distance. ${ }^{55}$ Furthermore, increased prevalence of comorbid conditions and obesity-specific disability, which are frequently observed in obese populations, might interfere with walking. ${ }^{72}$ Also, a number of volunteers, often termed as "healthy", may not correspond to criteria for being completely healthy and this can further contribute to the discrepancies in the 6MWD reference values.

Chapter 3 also demonstrated that besides those clinical characteristics, technical or methodological characteristics including different operational procedures, 6MWT instructions and encouragement can influence walking performance. It is reported that there is less walking effort in long corridors because of the smaller number of turns compared to short corridors $^{73}$ while lack of standardization in given instructions ${ }^{74}$ or encouragement ${ }^{75}$ can also affect 6MWD. Indeed, the standardization of the 6MWT is important for data interpretation as several factors may influence the outcome. In case that these factors are standardized and well controlled, the 6MWT can provide concrete valuable information. Moreover, other factors such as the regional habitual walking $^{76}$, psychological status ${ }^{76}$, and altered walking pattern ${ }^{77}$ as well as balance impairment $^{78}$ can partially determine 6MWD, while the relative weight of these determinants can differ. ${ }^{76}$ Clinicians should consider all factors that potentially influence the 6MWT for the right interpretation of the 6MWD reference values.

Herein, it should be also noted that the choice of right predicted equations is not $6 \mathrm{MWT}$-specific. Indeed, several reference equations exist in other clinical tests (i.e. $\mathrm{CPET}$, muscle function test, lung function, etc.). The findings of chapter 3 regarding to factors used for the formulation and application of reference equations may find a common ground with studies that deal with reference equations in other clinical tests. For instance, ERS/ATS interpretative strategies for lung function tests ${ }^{79}$ recommended taking the characteristics of age, sex, height, health status, ethnicity, equipment and general population characteristics into account when developing and updating reference equations. ${ }^{79} \mathrm{BMI}$ was also reported by another study as a factor that could influence lung function. ${ }^{80}$

A practical recommendation for clinicians and health professionals is to choose a reference equation that was formulated under same operating procedures and derived from a representative large population with a comparable range of age. It is noteworthy 
that an erroneous selection of reference equation may result in wrong estimation of actual exercise capacity and thus potentially misleading data into clinical decisionmaking process. Moreover, an underestimation or overestimation of actual exercise capacity of patients may have a negative impact on prescribed exercise protocols (i.e. misestimated exercise workloads) resulting in less efficient exercise interventions in COPD.

\section{THE PROGNOSTIC VALUE OF VARIABLES DERIVED FROM SIX-MINUTE WALK TEST}

A significant relationship between 6MWD and mortality or hospitalization has been demonstrated in literature. ${ }^{81-83}$ Several thresholds for 6MWD have been proposed for the prediction of mortality ${ }^{4,81,84-94}$ with a pooled mean of 317 meters indicating an increased risk for mortality in COPD. The range of disparity in the 6MWD may be attributed to the differences in studied populations, sources of recruitment, and different methodological and statistical approaches as well as the heterogeneity of disease which all may influence 6MWD (Figure 1).

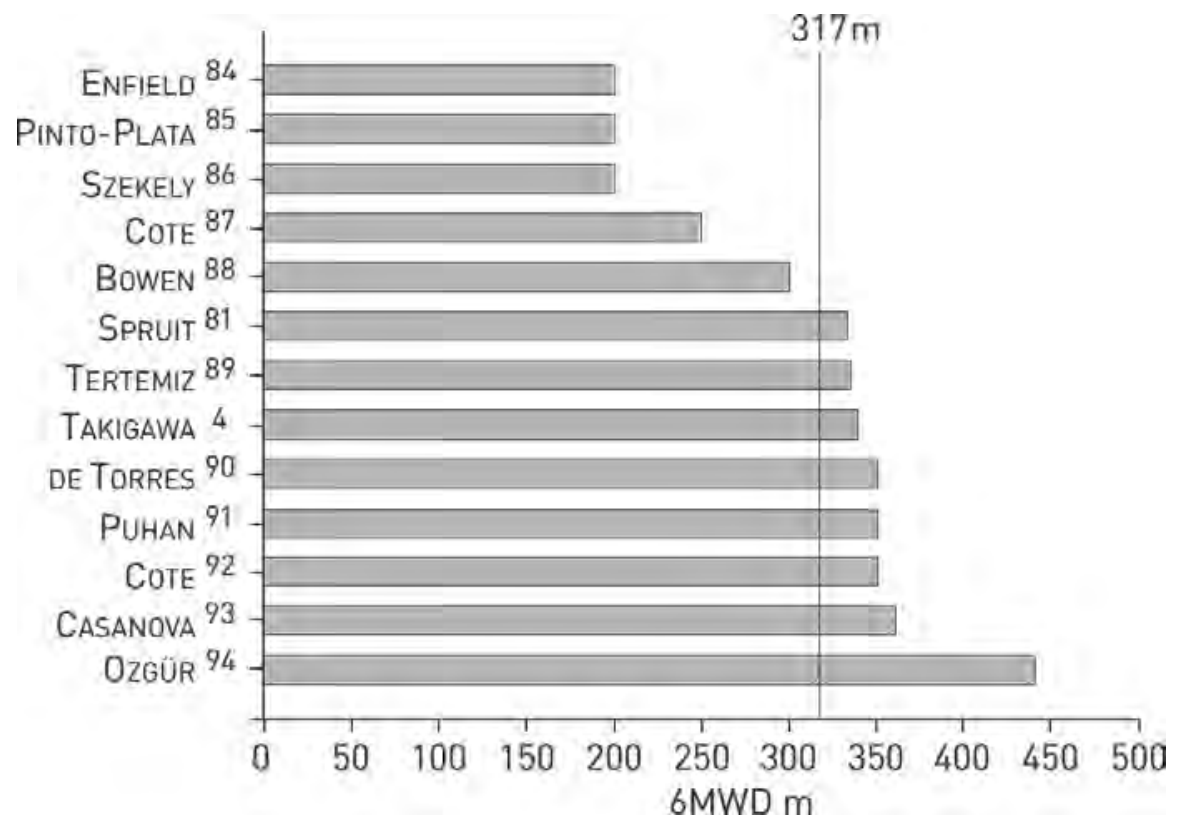

Figure 1. Thresholds of 6MWD for the prediction of mortality in COPD.

Vertical lines represent weighted mean thresholds for 6MWD across studies for predicting mortality. ${ }^{1}$ Reproduced with permission of the European Respiratory Society (C). European Respiratory Journal Dec 2014, 44 (6) 1447-1478; DOI: 10.1183/09031936.00150414. 
Chapter 4 expanded the prognostic value of 6MWT beyond the previous studies of the ECLIPSE cohort ${ }^{16,81,95}$ and establishes thresholds of 6MWT derived-variables for the prediction of mortality and hospitalization. In specific, 6MWT-derived variables including 6MWD, walking speed (6MWSpeed), six-minute walk-work (6MWW), distance saturation products (DSP), exercise-induced desaturation (EID), and unintended stop(s) demonstrated to be important predictors of mortality and hospitalization in patients with COPD. ${ }^{96}$ An adjusted logistical model indicated the variable of DSP as the strongest predictor of mortality and the BODE index as the most sensitive tool for assessing hospitalization in COPD. Moreover, unintended stop(s) during the 6MWT showed a significant prognostic value regarding the long-term prediction of mortality. ${ }^{96}$

Besides the 6MWD, the use and evaluation of several 6MWT-derived variables increases the total prognostic value of the 6MWT and may facilitate the clinical decision-making process. Specifically, several 6MWT-derived variables could be taken into account and assist clinicians in selecting patients who could most benefit from a clinical intervention and thus substantially increase life expectancy. Indeed, the appropriate selection of patients plays a key role in the success of a therapeutic intervention and the 6MWT-derived variables could be evaluated in this direction. It is known that patients with reduced exercise capacity who experience less ventilatory limitation to exercise and more reduced respiratory and peripheral muscle strength could be more likely to improve with exercise training. ${ }^{97}$ Subsequently, it may be reasonable that among patients with similarly low 6MWD, those with higher DSP, 6MWW and without unintended stop(s) are more likely to benefit from an exercise intervention and, therefore, may have better prognosis. Nevertheless, future research is needed to investigate the role of 6MWT-derived variables in the evaluation of the effect of a clinical intervention (such as a PR program, pharmacotherapy, chest surgery etc.) in terms of prognosis in COPD.

In accordance with previous studies ${ }^{81,98-100}$, the variables of 6MWD, 6MWSpeed, $6 \mathrm{MWW}, \mathrm{DSP}$, the BODE index ${ }^{101}$, and the presence of EID demonstrated an independent prognostic value on overall mortality and hospitalization. Next to the $6 \mathrm{MWD}$, the supplementary use of DSP and BODE index could be strongly advised to clinicians and health professionals as independent variables to estimate prognosis. Specifically, the 6MWW and DPS can be considered as multidimensional prognostic variables of the 6MWT combining walking distance with the effect of patients' body weight and oxygen desaturation during walking, respectively., ${ }^{49}$ Moreover, a statistical adjustment of those variables with a number of certain physiological and clinical characteristics including age, sex, BMI, FEV $1, F E V_{1} / F V C, S G R Q$, emphysema, and smoking, revealed also that DSP and BODE index remained significant and the strongest predictors of mortality and hospitalization, respectively.

Intriguingly, even though the BODE index was shown as the strongest predictor of hospitalization, it did not remain significant in the prediction of overall mortality after 
the aforementioned logistical adjustment. The crude association of the BODE index with mortality might likely fail to reflect a causal association due to confounding by the variable of SGRQ. ${ }^{102,103}$ This information is novel as previous studies did not included the variable of SGRQ in the adjustment of the BODE index.

Chapter 4 was also the first study demonstrating that the presence of unintended stop(s) during the 6MWT is an independent predictor of overall mortality and hospitalization. Previously, Wong and colleagues have reported that one in 5 individuals with chronic lung disease rest during the 6MWT. ${ }^{104}$ Several factors can intercept the constant walking of patients during the $6 \mathrm{MWT} .{ }^{105}$ Most common factor is the increased perception of dyspnea while a number of patients also report leg-fatigue as a limiting factor of walking capacity during the $6 \mathrm{MWT}{ }^{106}$ Interestingly, unintended stop(s), as an independent variable, remained significantly predictive of mortality into patients' groups assigned by impaired 6MWT-derived variables (6MWD $\leq 334 \mathrm{~m}$, 6MWSpeed $\leq 0.9 \mathrm{~m} / \mathrm{s}, 6 \mathrm{MWW} \leq 20000 \mathrm{~m} \mathrm{~kg}$, DSP $\leq 290 \mathrm{~m} \%$, EID) and by increased score of the BODE index ( $\geq 4$ points). Clinicians should be aware that unintended stop(s) during the 6MWT may reflect an overall impaired health status beyond the $\mathrm{FEV}_{1}$ or other respiratory indices and may be clinically relevant in medical decision making in regards to prognosis in COPD patients.

Herein, it is important to remark that findings of chapter 4 could have a clinical implication not only to patients with COPD but also to elderly with underlying comorbidities or to other clinical populations. Especially in the elderly characterized by an increased prevalence of comorbidities (most common cardiovascular, metabolic, rheumatologic or combination of thereof) significant differences can be found in functional exercise capacity according to health status ${ }^{107}$, that can be reflected in 6MWD, 6MWSpeed, 6MWW and unintended stop(s). Moreover, taking into consideration that both 6MWD and EID are valuable indicators of exercise capacity and mortality in various pulmonary and non-pulmonary diseases ${ }^{108-111}$, it is reasonable to hypothesise that 6MWT-derived variables as proposed in chapter 4, could be also evaluated in other clinical populations in which exercise capacity is associated with clinical manifestations. Indeed, the findings of chapter 4 could be used as a model to further explore the utility of 6MWT in patients or individuals that can be stratified based on physiological responses during walking.

\section{DETERMINANTS OF EXERCISE-INDUCED OXYGEN DESATURATION IN 6MWT AND THE ROLE OF PULMONARY EMPHYSEMA}

Exercise induced oxygen desaturation (EID) is frequently observed in patients with advanced COPD and is predictive of poorer clinical outcome. Several pathophysiological mechanisms and characteristics have been postulated to explain the occurrence of 
EID. ${ }^{112-117}$ Patients who exhibit EID usually present a variety of clinical findings including lower levels of $\mathrm{FEV}_{1}$ and DLCO ${ }^{114,115,117-120}$ with predominant emphysema phenotype ${ }^{121}$, worse blood gases $\left(\mathrm{PaO}_{2}\right)^{120,122,123}$ and higher dyspnea $(\mathrm{MRC})^{124}$ at rest compared to those without EID. A limited number of studies in COPD have shown a relationship between EID and pulmonary emphysema which is characterized by impairment or destruction of alveoli and lung tissue involved in gas exchange. ${ }^{125,126}$ Fewer alveoli, impaired lung tissue and damage to the pulmonary vasculature are associated with lower levels of DLCO and, therefore, less oxygen availability into the bloodstream which can result in ventilation/perfusion $\left(\mathrm{V}^{\prime} / \mathrm{Q}^{\prime}\right)$ mismatch. ${ }^{127-131}$ The inequality of $\mathrm{V}^{\prime} / \mathrm{Q}^{\prime}$ due to pulmonary emphysema and small airways disease, can be detected even in patients with mild COPD ${ }^{132}$, but appears to increase with the progress of disease severity. ${ }^{133}$

Chapter 5 showed that about $20 \%$ of subjects with clinically stable COPD, who were included in the ECLIPSE cohort, exhibited EID while occurrence of EID was associated with the degree of lung-density defined emphysema, severity of airflow limitation, baseline arterial oxygen saturation, and obesity. Almost half of the patients in GOLD stage IV and one out of 3 patients with severe to very severe pulmonary emphysema exhibited EID. Moreover, patients with EID in GOLD stage II had higher odds to have moderate or worse emphysema compared those with EID in GOLD stage III-IV. ${ }^{134}$

Patients with pulmonary emphysema usually present severe lung function impairment ${ }^{135,136}$ and particularly a decreased DLCO, which is attributed to the widespread loss of the pulmonary capillary bed. ${ }^{137,} 138$ Previously, a relationship between DLCO and the degree of emphysema on lung CT scans has been reported ${ }^{139,140}$ and a fair inverse correlation $(r=-0.63, p<0.001)$ between DLCO and the volume fraction of the lung with a density $<950$ Hounsfield units (i.e. percentage of lung affected by emphysema) has been demonstrated. ${ }^{141}$ The alveolar tissue destruction as well as $V^{\prime} / Q^{\prime}$ inequality can contribute to the occurrence of EID. ${ }^{133,142}$ In chapter 5, the percentages of EID were progressively increased across the degrees of emphysema. However, pulmonary emphysema as a part of pathophysiology in COPD is confounded by the airflow limitation which also contributes to EID. The degrees of emphysema along with the severity of airflow limitation as defined by FEV $_{1}$ or GOLD stages can provide clinicians and health professionals with information about the risks of EID in patients with COPD.

Interestingly, obesity was also identified as an additional determinant of EID in COPD. In order to appreciate the impact of obesity on lung function and pulse oxymetry in COPD, it is important to understand the impact of excessive fat mass on these outcomes per se. Isolated obesity is associated with reduced static lung volumes and normal to supraphysiological diffusion capacity. ${ }^{143,144}$ Reduced ventilatory capacity may result in alveolar ventilation/perfusion inequalities and decreased resting oxygen saturation in isolated obesity. ${ }^{143}$ In patients with co-occurring obesity and COPD, the degree of static hyperinflation is less pronounced than in normal weight COPD patients 
with comparable $\mathrm{FEV}_{1}$, age and same sex ${ }^{145}$, while there is no relevant impact of increasing BMI on resting oxygen saturation. ${ }^{146}$ Maatman and colleagues using several well-implemented exercise testing protocols in COPD, observed a significantly more pronounced EID during walking in obese female COPD patients, compared to non-obese female COPD patients. ${ }^{25}$ No difference was observed in male patients or in the same patients when a cycling test, as weight-supported exercise, was performed. The results of the present study partially confirm this finding, suggesting that obesity is a risk factor for EID during weight-bearing exercise in COPD. The observed difference according to sex of patients warrants further investigation, but might be sex-related differences in excessive fat mass distribution. ${ }^{143}$

Chapter 5, also investigated for first time the role of the ADO index ${ }^{91}$ in predicting EID in COPD. The ADO index is a multidimensional index including age, dyspnea rate by $\mathrm{MRC}$ and $\mathrm{FEV}_{1}(\%)$. According to the findings of Chapter 5, the ADO index may be recommended as an additional indicative index for the risk of EID during 6MWT. Especially, emphysematous patients with high ADO index should be monitored for oxygen desaturation. ${ }^{134}$ In addition; chapter 5 demonstrated that a combination of clinical characteristics could be used to prioritize the assessment for the detection of EID in those patients who are at high risk of EID prior to the participation on PR program. Indeed, a combination of certain lung function characteristics and resting oxygen saturation for the prediction of EID revealed that the highest proportion of patients (81\%) who exhibit EID are characterized by moderate to very severe pulmonary emphysema, obesity, impaired $\mathrm{FEV}_{1}$ and low resting $\mathrm{SpO}_{2}$ pre-walk values. The use of statistical models including several patient characteristics, as has been already suggested $^{123}$, can be a useful approach for obtaining fast information along the clinical decision-making process in patients with COPD.

\section{EXERCISE-INDUCED OXYGEN DESATURATION IN NON-HYPOXEMIC PATIENTS}

Patients without hypoxemia at rest (non-hypoxemic) are characterized by baseline oxygen saturation more than $88 \%$. Oxygen saturation can be measured by pulse oxymetry $\left(\mathrm{SpO}_{2}\right)$, which is an indirect, non-invasive method for assessing arterial oxygen tension $\left(\mathrm{PaO}_{2}\right)$ and can detect EID. ${ }^{147,148}$ EID means that some non-hypoxemic patients with COPD are exposed intermittently to hypoxemia $\left(\mathrm{SpO}_{2} \leq 88 \%\right)$ when performing exercise. Besides the increase of 6MWD, improvements in COPD can be manifested by attenuation of exercise-induced decline of $\mathrm{SpO}_{2}$ preventing thus the occurrence of EID. ${ }^{149}$

Chapter 6 revealed that baseline $\mathrm{SpO}_{2}$ cannot predict accurately the EID. A threshold of $95 \%$ at baseline $\mathrm{SpO}_{2}$ is not adequate to detect a potential EID during the 6MWT in 
patients with COPD, as was suggested by Knower and colleagues. ${ }^{150}$ However, the inclusion of additional patients' characteristics (such as sex, FEV 1, DLCO, and baseline $\mathrm{PaO}_{2}$ ) found to refine the prediction of EID. It was demonstrated that $39 \%$ of nonhypoxemic patients with COPD with GOLD stages II-IV, who were referred for Pulmonary Rehabilitation (PR), exhibited EID during the 6MWT. ${ }^{151}$

The detection of EID is useful for prognosis but can also provide clinicians information on the $\mathrm{SpO}_{2}$ trend during daily life of patients with COPD. Evidence has shown that patients who exhibit EID during the first minute of the 6MWT, the "early desaturators", have increased risk to desaturate during activities of daily living. In contrast, those who desaturate after the 3.5 minutes in the 6MWT, the "late desaturators", have lower risk to manifest desaturation in their daily life activities. ${ }^{152}$ Moreover, non-hypoxemic patients at rest but with substantial and more rapid EID during the 6MWT will require Long-Term Oxygen Therapy (LTOT) sooner than those with a more late-onset EID. Nowadays, a decline of $\mathrm{SpO}_{2} \leq 88 \%$ (often followed by a reduction of $\geq 4 \%$ from the baseline) during physical activity is considered as an indication for prescription and reimbursement of continuous oxygen use by Centres for Medicare \& Medicaid Services in patients with COPD who are non-hypoxemic at rest. Therefore, the $39 \%$ of patients in chapter 6 , who exhibited EID, may be suitable candidates for LTOT. ${ }^{153}$ This higher prevalence of EID compared to the ECLIPSE cohort (39\% vs 21\%) may be explained by the fact that those patients were referred for PR program, which indicates that they had more evident symptoms of COPD.

The correction of EID during exercise or physical activity by LTOT can result in alleviation of dyspnea, increase of physical condition and might contribute to more effective PR programs by allowing more intensive exercise training. ${ }^{154,155}$ However, the question of whether or not clinicians should administer oxygen to patients at home cannot be easily answered from a 6MWT solely. For instance, a patient with EID during the 6MWT may not reach desaturation at home in case of low intensity physical activity. Indeed, a number of patients may not present EID outside the hospital even though they were detected with EID during the 6MWT. ${ }^{156}$ In these cases, $\mathrm{SpO}_{2}$ measurement during laboratory or field exercise tests is not representative of the $\mathrm{SpO}_{2}$ in the home setting. However, only the possibility of functional and clinical improvements (such as exercise tolerance, quality of life, survival and hospitalization rate) can provide an important incentive for the use of LTOT in patients with COPD. ${ }^{157}$ Considering also that evidence of "harm" induced by relatively modest fraction of inspired oxygen $\left(\mathrm{FiO}_{2}\right)$ is absent, the LTOT remains recommended. ${ }^{158}$

The detection of EID in clinical settings remains important because it is associated with higher mortality rates ${ }^{4}$ 98, 99, 159 and impaired daily physical activity. ${ }^{160}$ The suggested methodological approach for obtaining rapid information about the occurrence of EID after considering an alarm $\mathrm{SpO}_{2}$ threshold of $95 \%$ in pulse oxymetry at baseline ${ }^{150}$ is not accurate according to the findings of chapter 6 . In fact, measuring 
oxygen saturation by pulse oxymetry is subject to important limitations. ${ }^{161}$ For instance, values of pulse oxymetry generally have a margin of error \pm 2 points of the actual $\mathrm{SpO}_{2}$ levels and very low blood pressure can affect the pulse oxymetry readings. ${ }^{162-164}$ Furthermore, oxygen saturation may be overestimated in heavy smokers because of high carboxyhaemoglobin levels. ${ }^{165}$ In addition, baseline levels of oxygen saturation seems to be independent of the degree of airways obstruction, as quantified by the $\mathrm{FEV}_{1}{ }^{166}$ On the other hand, $\mathrm{FEV}_{1}$ is associated with dynamic hyperinflation ${ }^{167}$ which, in turns, is related to EID. ${ }^{168}$ Low FEV $_{1}(<45 \%)$ seems also to be associated with the magnitude of $\mathrm{SpO}_{2}$ decline during exercise according to the findings of chapter 6 . Therefore, clinicians should not solely rely on baseline $\mathrm{SpO}_{2}$ values of pulse oxymeter but they should also consider other clinical characteristics, such as FEV 1 , DLCO, baseline $\mathrm{PaO}_{2}$ in respect to EID as suggested in chapter 6.

An additional question that arises is whether clinicians should use the lowest or the end value of $\mathrm{SpO}_{2}$ during the 6MWT for the detection of EID. It is demonstrated that the end value of $\mathrm{SpO}_{2}$ may lead to an underestimation of the number of patients with EID. Some patients, who have marginally reached $\mathrm{SpO}_{2} \leq 88 \%$ during $6 \mathrm{MWT}$, may perform unintended stop(s) during the 6MWT or potentially reduce the effort at the end of the exertion allowing a small increase of $\mathrm{SpO}_{2}$ levels above the threshold of $88 \%$ which defines the EID. Even though these cases are not prevalent, the lowest value of $\mathrm{SpO}_{2}$ should be ideally used for the detection of all patients with potential EID. Therefore, the continuous recording of $\mathrm{SpO}_{2}$ during the $6 \mathrm{MWT}$ should be recommended.

In chapter 6, a predictive logistical model of EID including variables of FEV 1 , DLCO, $\mathrm{PaO}_{2}$ and baseline $\mathrm{SpO}_{2}$ was used to detect high risk patients for EID. Interestingly, sex had also an influence on the occurrence of EID: female gender was associated with more prevalent EID during the 6MWT. According to physiological characteristics of women compared to men, women have smaller lungs, reduced lung diffusion (DLCO), and lower maximal oxygen consumption $\left(\mathrm{VO}_{2} \max \right)$ compared to age- and heightmatched men. ${ }^{169}$ Furthermore, a study determining reference values for arterial blood gases has demonstrated higher values of $\mathrm{PaO}_{2}$ in elderly men than in elderly women. ${ }^{170}$ This could only partly explain the influence of sex on EID. However, further studies are needed to reproduce and explain these sex-related differences in EID.

\section{CARBON-DIOXIDE PARTIAL PRESSURE IN 6MWT AND THE EXERCISE- INDUCED HYPERCAPNIA}

Transcutaneous carbon dioxide partial pressure $\left(\mathrm{T}_{\mathrm{C}} \mathrm{PCO}_{2}\right)$ can be reliably measured providing indirect information on alveolar ventilation performance. ${ }^{171-174}$ Patients with COPD may exhibit $\mathrm{CO}_{2}$ retention during exercise to hypercapnic $\mathrm{CO}_{2}$ levels $\left(\mathrm{PtcCO}_{2}\right.$ $>46 \mathrm{mmHg}$ ). Exercise-induced hypercapnia $(\mathrm{EIH})$ can reflect integrated alterations in 
minute ventilation (VE), physiologic dead space (VD/VT), and carbon dioxide output as determined by alveolar ventilation. ${ }^{122}$

Chapter 7 demonstrated a high heterogeneity of $\mathrm{T}_{C} \mathrm{PCO}_{2}$ levels in response to $6 \mathrm{MWT}$. Patients who exhibited a substantial $\mathrm{CO}_{2}$ retention $\left(\mathrm{T}_{\mathrm{C}} \mathrm{PCO} 2[\Delta]>4 \mathrm{mmHg}\right.$ from baseline) during exercise had higher pre-walk dyspnea and worse airflow limitation $\left(\mathrm{CO}_{2}\right.$ retainers). Patients who reached the hypercapnic $\mathrm{CO}_{2}$ levels during exercise $(\mathrm{EIH}$ : $\mathrm{T}_{\mathrm{C}} \mathrm{PCO} 2>46 \mathrm{mmHg}$ ) were characterized by higher pre-walk dyspnea and increased baseline levels of capillary carbon-dioxide pressure $\left(\mathrm{PCCO}_{2}\right)$. In addition, it seems that overweight-BMI has a protective effect against EIH in very severe COPD. ${ }^{175}$

In chapter 7 , a high variability in the development of $\mathrm{T}_{\mathrm{C}} \mathrm{PCO}_{2}$ during the transition from rest to exercise was detected in agreement with previous study. ${ }^{122}$ O 'Donnell and colleagues reported that that retention of $\mathrm{CO}_{2}$ often occurs during exercise in advanced COPD, even when is not present at rest, and it can be a sign of inadequate ventilation. ${ }^{122} \mathrm{CO}_{2}$ retention and/or EIH during the 6MWT could also imply a number of certain pathophysiological conditions such as dynamic hyperinflation, reduced gas exchange capabilities (ventilation/perfusion inequality), ventilatory abnormalities such as increased VD/VT, and more rarely the opening of right to left shunt. ${ }^{122,176}$ Regardless the factors that trigger $\mathrm{CO}_{2}$ retention, the increase of $\mathrm{PCO}_{2}$ during exercise amplifies dyspnea by increased activation of central chemoreceptors. The ability to deal with increasing $\mathrm{CO}_{2}$ during exercise is usually dependent on the degree of $\mathrm{FEV}_{1}$, ventilation rate and the inherent sensitivity to carbon dioxide. ${ }^{177,178}$

In chapter 7 , half of the patients (50\%) with very severe COPD demonstrated $\mathrm{CO}_{2}$ retention, whereas 31\% of total population exhibited EIH during the 6MWT. Exerciseinduced $\mathrm{CO}_{2}$ retention was found to be determined by very severe airflow limitation $\left(\mathrm{FEV}_{1} \leq 36 \%\right)$ and increased baseline dyspnea. Indeed, very impaired $\mathrm{FEV}_{1}$ can cause airtrapping generating dynamic hyperinflation which, in turns, is related with the occurrence of $\mathrm{CO}_{2}$ retention during exercise in COPD. ${ }^{179}$ At increased respiratory rates during exercise, an inversely proportional shortening in expiratory time may be occurred and lead to the development of dynamic hyperinflation in COPD. ${ }^{180}$ Dynamic hyperinflation can cause lung mechanic dysfunctions resulting in $\mathrm{CO}_{2}$ retention, while it has also been implicated as major determinant of dyspnea. ${ }^{181}$ Nevertheless, some patients with severely impaired $\mathrm{FEV}_{1}$ did not exhibit EIH. In chapter 7, the occurrence of $\mathrm{EIH}$ found to be much dependent on baseline $\mathrm{PCO}_{2}$ levels which are mainly related to impaired gas exchange and/or reduce drive to breathe at rest (low sensitivity to $\left.\mathrm{CO}_{2}\right) .{ }^{182}$, 183 Indeed, patients with increased baseline $\mathrm{PCO}_{2}$ levels ( $\geq 41 \mathrm{mmHg}$ ) had higher odds to exhibit EIH according to the findings of chapter 7.

Other factors such as inspiratory muscle weakness are also associated with $\mathrm{CO}_{2}$ retention which can lead to the occurrence of EIH in COPD. ${ }^{122,184}$ In chapter 7, patients with EIH presented lower peak inspiratory mouth-pressure ( $\mathrm{Pi}$ max) indicating greater inspiratory muscle weakness compared to non-EIH. Also, the evaluation of Pi max as 
determinant of $\mathrm{CO}_{2}$ retention and $\mathrm{EIH}$ showed a strong tendency towards statistical significance ( $p=0.051$ and $p=0.050$, respectively). It seems that $\mathrm{CO}_{2}$ retention due to muscle loading occurs at very low values of $\mathrm{Pi}$ max. Otherwise, inspiratory muscle weakness is associated with alveolar hypoventilation contributing to $\mathrm{CO}_{2}$ retention and EIH when the inspiratory loads are high due to static or dynamic hyperinflation. ${ }^{185}$ Particularly, the unbalanced inspiratory muscle loading-to-strength ratio at exertion likely due to impaired breathing pattern and/or the occurrence of dynamic hyperinflation can trigger the signal for the integrated response that leads to rapid and shallow breathing contributing to $\mathrm{EIH}$ in patients with COPD. ${ }^{185-187}$ Shallow breathing presumably represents a key protective mechanism to prevent respiratory muscle fatigue ("wise fighter" hypothesis), however at the price of an insufficient alveolar ventilation. $^{188,189}$ Mechanical factors may substantially constrain the ventilatory response to the metabolically generated $\mathrm{CO}_{2}$ in such a way that $\mathrm{PCO}_{2}$ increases (i.e., compromised respiratory compensation). ${ }^{190}$ Indeed, severe mechanical restriction secondary to dynamic hyperinflation and increased respiratory muscle work has been recognized as a contributory factor to EIH in COPD. ${ }^{122,142}$

In consistency with previous findings ${ }^{145,191-194}$, chapter 7 also demonstrated a protective role of overweight-BMI against the occurrence of EIH in patients with advanced COPD. Although the higher body surface area in overweight or obese patients can result in higher $\mathrm{CO}_{2}$ production as a result of large metabolically active muscle mass $^{195}$, it can also lead to lower static and dynamic hyperinflation and to lower mechanical loading during exercise. ${ }^{192,193}$ Moreover, the inspiratory capacity as related to total lung capacity (IC/TLC ratio) is higher in overweight or obese compared to patients with a normal range BMI. ${ }^{192,193}$ These mechanical effects in higher BMI reduce operating lung volumes at rest and throughout exercise ${ }^{145}$ and may have favorable influence on $\mathrm{PCO}_{2}$ levels, especially during effort. In addition, altered metabolic loading at exertion in patients with higher BMI can stimulate an increase in minute ventilation during exercise compared to those with normal-ranged BMI and thus may prevent the occurrence of ElH. ${ }^{196,197}$

Chapter 7 did not detect a relationship between $\mathrm{CO}_{2}$ retention/EIH and EID; however, it should be reported that the majority (79\%) of studied patients used oxygen during the 6MWT and this may have diminished the extent of $\mathrm{SpO}_{2}$ decline contributing to the lack of association between EIH and EID. Evidence has shown that changes in $\mathrm{T}_{\mathrm{C}} \mathrm{PCO}_{2}$ have a fair negative correlation with the magnitude of $\mathrm{SpO}_{2}$ decline. ${ }^{122}$ Changes in $\mathrm{T}_{\mathrm{C}} \mathrm{PCO}_{2}$ and $\mathrm{SpO}_{2}$ are dependent on global gas exchange, cardiac output, oxygen content and are also influenced by regional blood flow and oxygen content. ${ }^{198}$ Nevertheless, different shapes of the oxygen and carbon dioxide dissociation curves determine the peak and lowest values of $\mathrm{T}_{\mathrm{C}} \mathrm{PCO}_{2}$ and $\mathrm{SpO}_{2}{ }^{199}$ Also, the use of supplemental oxygen in the vast majority of studied patients may diminish the extent of $\mathrm{SpO}_{2}$ decline and thus the presence of EID and this may attributed for the lack of 
association between $\mathrm{CO}_{2}$ retention, $\mathrm{EIH}$ and the occurrence of EID. Chapter 7 also indicated that $\mathrm{CO}_{2}$ retention and $\mathrm{EIH}$ do not have significant impact on the 6MWD. This is in consistence with the observation of Montes de Oca and Celli ${ }^{178}$ who reported that exercise capacity is similarly reduced in eucapnic and hypercapnic patients with severe stable COPD, even though hypercapnic patients achieve lower ventilation during exercise. $^{178}$

Interpreting the findings of Chapter 7 from a clinical perspective, the occurrence of $\mathrm{CO}_{2}$ retention and $\mathrm{EIH}$ during the 6MWT may represent a marker of disease severity that is beyond the walking capacity. In other words, the case of a patient who did not improve significantly the 6MWD but prevented the high accumulation of $\mathrm{CO}_{2}$ and/or the occurrence of EIH in 6MWT should be considered as beneficial. Less increase in $\mathrm{PCO}_{2}$ levels during 6MWT may reflect improvement in respiratory capability at exertion, which, in turns, can be related to a better prognosis, irrespectively from the distance walked. Therefore, monitoring of $\mathrm{T}_{\mathrm{C}} \mathrm{PCO}_{2}$ during walking, especially in severe COPD could be recommended as it may provide clinicians with additional clinical information. Moreover, monitoring of $\mathrm{T}_{\mathrm{C}} \mathrm{PCO}_{2}$ during walking could also be useful to evaluate the impact of inspiratory muscle training (IMT) and/or the effect of non-invasive ventilation (NIV) to counteract respiratory muscle weakness and $\mathrm{EIH}$. Furthermore, a future perspective on exercise-induced $\mathrm{PCO}_{2}$ changes from baseline levels during the 6MWT or other exercise test in respect to mortality, hospitalization, and other deteriorations such as cognitive deficits during the progress of disease severity, can be suggested for COPD patients.

\section{METHODOLOGICAL CONSIDERATIONS}

The present thesis has some important limitations that should be considered in interpreting the results. As general drawback, this thesis includes some retrospective cohort studies (Chapters 2, 3, 4, 5) which were constructed from databases of healthcare records that have been collected in the past. There were a few consequences attributed to the retrospective collection of the data. Some of the records were incomplete without knowing the exact reason of lacking data, which may be either due to the failure of the patient to complete the initial PR assessment or due to the refusal to perform a particular test. Some were also repeat evaluations. Moreover, many different healthcare professionals would have been involved in data recording and therefore the measurement of risk factors and outcomes would probably be not as highly accurate as in a prospective research design. Furthermore, the retrospective analyses in this thesis are subject to unidentified confounders associated with particular tests and/or evaluations. Also, selection bias could occurred if the selected cohorts were not representative of all possible patients with COPD. ${ }^{200}$ 
However, the large size of the cohorts analysed retrospectively in this thesis reduces the confounding effects.

There are also certain limitations regarding to the specific aims of each chapter included in this thesis. In chapter 2, it should be noted that the inclusion of patients' population referred for PR program could result in a referral bias towards patients with a greater degree of limitation in clinical care. Nevertheless, the fixed proportion of WR peak in exercise assessment does not provide identical physiological stress among patients and this would be also similar to the patients with more severe COPD. In chapters 4 and 5 , a critical criticism could focus on the fact that patients performed only one 6MWT instead of two as recommended by ERS/ATS technical standards. ${ }^{1,2}$ This fact may have resulted in a slightly lower 6MWD, as usually the second 6MWT is longer in about $80 \%$ of patients with COPD $^{1}$, and consequently in lower 6MWT-derived outcomes (6MWW, DSP) and changes in $\mathrm{SpO}_{2}$ during walking. Furthermore, the EID was defined based on the $\mathrm{SpO}_{2}$ at the end of the 6MWT and not the $\mathrm{SpO}_{2}$ nadir for both chapters 4 and 5, which can lead to an underestimation of the number of patients who exhibit EID. However, this bias is believed that is relatively low as $\mathrm{SpO}_{2}$ at the end of the 6MWT is similar with the nadir values with differences ranging from $1 \%$ to $10 \%{ }^{201}$ Chapter 6 included patients consisted of a convenience sample, while in chapter 7 there was no possibility to acquire respiratory measurements such as ventilation, tidal flowvolume loops, end-tidal $\mathrm{PCO}_{2}$ and thus the detection of dynamic hyperinflation were not performed during walking but only assumed. Moreover, there was no detailed assessment of dynamic lung mechanics, which would have provided us with important information for patients' respiratory profile during the 6MWT.

Despite these methodological limitations, this thesis contributes with important clinical information about the 6MWT and the 6MWT-derived variables. Due to the large size populations, which are included in analyses, the findings of each chapter could lead to concrete conclusion that enhance the use of the 6MWT in daily clinical routine.

\section{CONCLUSIONS, FUTURE PERSPECTIVES AND RECOMMENDATIONS FOR RESEARCH}

In summary, this thesis has shown that 6MWT is a useful clinical tool providing important clinical information well beyond the measure of 6MWD. It is sufficient to provide concrete exercise outcomes based on multidimensional 6MWT-derived variables and to detect the occurrence of oxygen desaturation better than CET. Additionally, the 6MWT has a considerable prognostic value which is enhanced using not only the 6MWD but also other 6MWT-derived variables. Clinicians and health professionals can have a robust prediction in regards to mortality and hospitalization evaluating the DSP and BODE index, respectively. Moreover, the understanding of the 
determinant of EID during the 6MWT and the role of pulmonary emphysema in oxygen desaturation can increase the interpretation of the occurrence of EID during 6MWT. The pathophysiological mechanism of EID can be caused by low FEV 1 , DLCO, and baseline $\mathrm{PaO}_{2}$ when the metabolic demands are increasing during walking. On the other hand, EID in patients with mild COPD could imply the existence of pulmonary emphysema. Clinically significant information about the respiratory impairment can also derived from the monitoring of $\mathrm{PCO}_{2}$ during $6 \mathrm{MWT}$ and the detection of $\mathrm{PCO}_{2}$ trends as an additional marker of disease severity. Indeed, the utilization of all the clinical information that 6MWT is possible to provide is crucial for the clinical decision-making process in COPD.

Future research is necessary to be able to acquire all the possible clinical information from a $6 \mathrm{MWT}$. Indeed, this thesis increased the utility of the 6MWT by expanding the knowledge for several 6MWT-derived variables. Some of them were already considered in previous research studies but some also were newly analyzed and proposed by this thesis. A step forward has been done; however, more future research is needed especially regarding variable such as the levels of $\mathrm{PCO}_{2}$ during the 6MWT. The changes in $\mathrm{PCO}_{2}$ during the 6MWT constitute a variable that is usually neglected in clinical settings. The constant monitoring of $\mathrm{PCO}_{2}$ during the 6MWT is rarely performed even though it could provide clinicians with important information about the respiratory sufficiency and detect patients at high risk for $\mathrm{EIH}$. Moreover, $\mathrm{PCO}_{2}$ is the fundamental regulator of cerebral blood flow and, therefore, disturbance of its levels during the 6MWT could be also related to the increased rates of cognitive dysfunction in COPD. Indeed, $\mathrm{PCO}_{2}$ trends or changes in several multidimensional variables derived from a 6MWT may be able to determine not only the risks for mortality or hospitalization but also the risk for the development of certain comorbidities. 


\section{REFERENCES}

1. Singh SJ, Puhan MA, Andrianopoulos V, et al. An official systematic review of the European Respiratory Society/American Thoracic Society: measurement properties of field walking tests in chronic respiratory disease. Eur Respir J. 2014; 44: 1447-78.

2. Holland AE, Spruit MA, Troosters $T$, et al. An official European Respiratory Society/American Thoracic Society technical standard: field walking tests in chronic respiratory disease. Eur Respir J. 2014; 44: 142846.

3. Gloeckl R, Marinov B and Pitta F. Practical recommendations for exercise training in patients with COPD. Eur Respir J. 2013; 22: 178-86.

4. Takigawa N, Tada A, Soda R, et al. Distance and oxygen desaturation in 6-min walk test predict prognosis in COPD patients. Respir Med. 2007; 101: 561-7.

5. Pichurko BM. Exercising your patient: which test(s) and when? Respir Care. 2012; 57: 100-10

6. Pitta F, Troosters T, Spruit MA, Probst VS, Decramer M and Gosselink R. Characteristics of physical activities in daily life in chronic obstructive pulmonary disease. Am J Respir Crit Care Med. 2005; 171: 972-7.

7. Starobin D, Kramer MR, Yarmolovsky A, et al. Assessment of functional capacity in patients with chronic obstructive pulmonary disease: Correlation between cardiopulmonary exercise, 6 minute walk and 15 step exercise oximetry test. Isr Med Assoc J. 2006; 8: 460-3.

8. Borel B, Provencher S, Saey D and Maltais F. Responsiveness of various exercise-testing protocols to therapeutic interventions in COPD. Pulm Med. 2013; 2013: 410748.

9. Andrianopoulos V, Wagers SS, Groenen MT, et al. Characteristics and determinants of endurance cycle ergometry and six-minute walk distance in patients with COPD. BMC Pulm Med. 2014; 14: 97

10. Buford TW, MacNeil RG, Clough LG, et al. Active muscle regeneration following eccentric contractioninduced injury is similar between healthy young and older adults. J App/ Physiol (1985). 2014; 116: 148190.

11. Shephard RJ. Maximal oxygen intake and independence in old age. Br J Sports Med. 2009; 43: 342-6.

12. Marcell TJ. Sarcopenia: causes, consequences, and preventions. J Gerontol A Biol Sci Med Sci. 2003; 58: M911-6.

13. Murtagh EM, Murphy MH, Murphy NM, Woods C, Nevill AM and Lane A. Prevalence and correlates of physical inactivity in community-dwelling older adults in Ireland. PLoS One. 2015; 10: e0118293.

14. Kobayashi A, Yoneda T, Yoshikawa $M$, et al. The relation of fat-free mass to maximum exercise performance in patients with chronic obstructive pulmonary disease. Lung. 2000; 178: 119-27.

15. Ischaki E, Papatheodorou G, Gaki E, Papa I, Koulouris N and Loukides S. Body mass and fat-free mass indices in COPD: Relation with variables expressing disease severity. Chest. 2007; 132: 164-9.

16. Spruit MA, Watkins ML, Edwards LD, et al. Determinants of poor 6-min walking distance in patients with COPD: the ECLIPSE cohort. Respir Med. 2010; 104: 849-57.

17. Farkhooy A, Janson C, Arnardottir RH, Malinovschi A, Emtner M and Hedenstrom H. Impaired carbon monoxide diffusing capacity is the strongest predictor of exercise intolerance in COPD. COPD 2013; 10: 180-5.

18. Mohsenifar Z, Lee SM, Diaz P, et al. Single-breath diffusing capacity of the lung for carbon monoxide: a predictor of $\mathrm{PaO}_{2}$, maximum work rate, and walking distance in patients with emphysema. Chest. 2003; 123: $1394-400$

19. Mador MJ, Mogri M and Patel A. Contractile fatigue of the quadriceps muscle predicts improvement in exercise performance after pulmonary rehabilitation. J Cardiopulm Rehabil Prev. 2014; 34: 54-61.

20. Mahler DA, Faryniarz K, Tomlinson D, et al. Impact of dyspnea and physiologic function on general health status in patients with chronic obstructive pulmonary disease. Chest. 1992; 102: 395-401.

21. Bentsen SB, Henriksen AH, Wentzel-Larsen T, Hanestad BR and Wahl AK. What determines subjective health status in patients with chronic obstructive pulmonary disease: importance of symptoms in subjective health status of COPD patients. Health Qual Life Outcomes. 2008; 6: 115. 
22. Agrawal MB and Awad NT. Correlation between Six Minute Walk Test and Spirometry in Chronic Pulmonary Disease. J Clin Diagn Res. 2015; 9: OC01-4.

23. Sava F, Laviolette L, Bernard S, Breton MJ, Bourbeau J and Maltais F. The impact of obesity on walking and cycling performance and response to pulmonary rehabilitation in COPD. BMC Pulm Med. 2010; 10: 55.

24. Capodaglio P, De Souza SA, Parisio C, et al. Reference values for the 6-Min Walking Test in obese subjects. Disabil Rehabil. 2013; 35: 1199-203.

25. Maatman RC, Spruit MA, van Melick PP, et al. Effects of obesity on weight-bearing versus weightsupported exercise testing in patients with COPD. Respirology 2016; 21: 483-8.

26. Pepin V, Saey D, Whittom F, LeBlanc $P$ and Maltais F. Walking versus cycling: sensitivity to bronchodilation in chronic obstructive pulmonary disease. Am J Respir Crit Care Med. 2005; 172: 151722.

27. Morgan MD and Singh SJ. Assessing the exercise response to a bronchodilator in COPD: time to get off your bike? Thorax. 2007; 62: 281-3.

28. Laviolette L, O'Donnell DE, Webb KA, Hamilton AL, Kesten S and Maltais F. Performance during constant workrate cycling exercise in women with COPD and hyperinflation. COPD. 2009; 6: 340-51.

29. Jones PW, Quirk FH, Baveystock CM and Littlejohns P. A self-complete measure of health status for chronic airflow limitation. The St. George's Respiratory Questionnaire. Am Rev Respir Dis. 1992; 145: 1321-7.

30. De Torres JP, Casanova C, Hernandez C, Abreu J, Aguirre-Jaime A and Celli BR. Gender and COPD in patients attending a pulmonary clinic. Chest. 2005; 128: 2012-6.

31. Mainguy $\mathrm{V}$, Malenfant $\mathrm{S}$, Neyron AS, et al. Alternatives to the six-minute walk test in pulmonary arterial hypertension. PLoS One. 2014; 9: e103626.

32. Vivodtzev I, Gagnon P, Pepin V, et al. Physiological correlates of endurance time variability during constant-workrate cycling exercise in patients with COPD. PLoS One. 2011; 6: e17007.

33. Howald H, Pette D, Simoneau JA, Uber A, Hoppeler H and Cerretelli P. Effect of chronic hypoxia on muscle enzyme activities. Int J Sports Med. 1990; 11 Suppl 1: S10-4.

34. Corbucci GG, Menichetti A, Cogliati A and Ruvolo C. Metabolic aspects of cardiac and skeletal muscle tissues in the condition of hypoxia, ischaemia and reperfusion induced by extracorporeal circulation. Int J Tissue React. 1995; 17: 219-25.

35. Mador MJ, Wendel T and Kufel TJ. Effect of acute hypercapnia on diaphragmatic and limb muscle contractility. Am J Respir Crit Care Med. 1997; 155: 1590-5.

36. Vianna LG, Koulouris N, Lanigan C and Moxham J. Effect of acute hypercapnia on limb muscle contractility in humans. J Appl Physiol (1985). 1990; 69: 1486-93.

37. Mador MJ and Bozkanat E. Skeletal muscle dysfunction in chronic obstructive pulmonary disease. Respir Res. 2001; 2: 216-24.

38. Puente-Maestu L, Palange $P$, Casaburi R, et al. Use of exercise testing in the evaluation of interventional efficacy: an official ERS statement. Eur Respir J. 2016; 47: 429-60.

39. Spruit MA, Augustin IM, Vanfleteren LE, et al. Differential response to pulmonary rehabilitation in COPD: multidimensional profiling. Eur Respir J. 2015; 46: 1625-35.

40. Celli BR, MacNee W and Force AET. Standards for the diagnosis and treatment of patients with COPD: a summary of the ATS/ERS position paper. Eur Respir J. 2004; 23: 932-46.

41. Vestbo J, Hurd SS, Agusti AG, et al. Global Strategy for the Diagnosis, Management, and Prevention of Chronic Obstructive Pulmonary Disease: GOLD Executive Summary. Am J Respir Crit Care Med. 2013; 187: 347-65.

42. NICE. Chronic obstructive pulmonary disease. National clinical guideline on management of chronic obstructive pulmonary disease in adults in primary and secondary care. Thorax. 2004; 59 Suppl 1: 1-232.

43. Fotheringham I, Meakin G, Punekar YS, Riley JH, Cockle SM and Singh SJ. Comparison of laboratory- and field-based exercise tests for COPD: a systematic review. Int J Chron Obstruct Pulmon Dis. 2015; 10: 62543. 


\section{Chapter 8}

44. Troosters T, Gosselink R and Decramer M. Six-minute walking distance in healthy elderly subjects. Eur Respir J. 1999; 14: 270-4.

45. Lee WTN, Peacock AJ and Johnson MK. The role of per cent predicted 6-min walk distance in pulmonary arterial hypertension. Eur Respir J. 2010; 36: 1294-301.

46. Holland A, Spruit MA and Singh S. How to carry out a field walking test in chronic respiratory disease. Breathe. 2015; 11: 128-39.

47. Andrianopoulos V, Holland AE, Singh SJ, et al. Six-minute walk distance in patients with chronic obstructive pulmonary disease: Which reference equations should we use? Chron Respir Dis. 2015; 12: 111-9.

48. Camarri B, Eastwood PR, Cecins NM, Thompson PJ and Jenkins S. Six minute walk distance in healthy subjects aged 55-75 years. Respir Med. 2006; 100: 658-65.

49. Gibbons WJ, Fruchter N, Sloan S and Levy RD. Reference values for a multiple repetition 6-minute walk test in healthy adults older than 20 years. J Cardiopulm Rehabil. 2001; 21: 87-93.

50. Chetta A, Zanini A, Pisi G, et al. Reference values for the 6-min walk test in healthy subjects 20-50 years old. Respir Med. 2006; 100: 1573-8.

51. Poh H, Eastwood PR, Cecins NM, Ho KT and Jenkins SC. Six-minute walk distance in healthy Singaporean adults cannot be predicted using reference equations derived from Caucasian populations. Respirology. 2006; 11: 211-6.

52. Enright PL, McBurnie MA, Bittner $\mathrm{V}$, et al. The 6-min walk test: a quick measure of functional status in elderly adults. Chest. 2003; 123: 387-98.

53. Kim AL, Kwon JC, Park I, et al. Reference equations for the six-minute walk distance in healthy korean adults, aged 22-59 years. Tuberc Respir Dis. 2014; 76: 269-75.

54. Dourado VZ, Vidotto MC and Guerra RL. Reference equations for the performance of healthy adults on field walking tests. J Bras Pneumol. 2011; 37: 607-14.

55. Enright PL and Sherrill DL. Reference equations for the six-minute walk in healthy adults. Am J Respir Crit Care Med. 1998; 158: 1384-7.

56. Masmoudi K, Aouicha MS, Fki H, Dammak J and Zouari N. [The six minute walk test: which predictive values to apply for Tunisian subjects aged between 40 and 80 years?]. Tunis Med. 2008; 86: 20-6.

57. Jenkins S, Cecins N, Camarri B, Williams C, Thompson P and Eastwood P. Regression equations to predict 6-minute walk distance in middle-aged and elderly adults. Physiother Theory Pract. 2009; 25: 516-22.

58. Iwama AM, Andrade GN, Shima P, Tanni SE, Godoy I and Dourado VZ. The six-minute walk test and body weight-walk distance product in healthy Brazilian subjects. Braz J Med Biol Res. 2009; 42: 1080-5.

59. Ben Saad H, Prefaut C, Tabka Z, et al. 6-minute walk distance in healthy North Africans older than 40 years: influence of parity. Respir Med. 2009; 103: 74-84.

60. Alameri H, Al-Majed S and Al-Howaikan A. Six-min walk test in a healthy adult Arab population. Respir Med. 2009; 103: 1041-6.

61. Osses AR, Yanez VJ, Barria PP, et al. [Reference values for the 6-minutes walking test in healthy subjects 20-80 years old]. Rev Med Chil. 2010; 138: 1124-30.

62. Soares MR and Pereira CA. Six-minute walk test: reference values for healthy adults in Brazil. J Bras Pneumol. 2011; 37: 576-83.

63. Casanova C, Celli BR, Barria P, et al. The 6-min walk distance in healthy subjects: reference standards from seven countries. Eur Respir J. 2011; 37: 150-6.

64. Hill K, Wickerson LM, Woon LJ, et al. The 6-min walk test: responses in healthy Canadians aged 45 to 85 years. Appl Physiol Nutr Metab. 2011; 36: 643-9.

65. Britto RR, Probst VS, de Andrade AF, et al. Reference equations for the six-minute walk distance based on a Brazilian multicenter study. Braz J Phys Ther. 2013; 17: 556-63.

66. Palaniappan Ramanathan $\mathrm{R}$ and Chandrasekaran B. Reference equations for 6 -min walk test in healthy Indian subjects (25-80 years). Lung India. 2014; 31: 35-8.

67. Doherty TJ. Invited review: Aging and sarcopenia. J Appl Physiol (1985). 2003; 95: 1717-27.

68. Bohannon RW. Population representative gait speed and its determinants. J Geriatr Phys Ther. 2008; 31: 49-52. 
69. Browning RC and Kram R. Energetic cost and preferred speed of walking in obese vs. normal weight women. Obes Res. 2005; 13: 891-9.

70. Beavers KM, Beavers DP, Houston DK, et al. Associations between body composition and gait-speed decline: results from the Health, Aging, and Body Composition study. Am J Clin Nutr. 2013; 97: 552-60.

71. Monteiro F, Camillo CA, Vitorasso R, et al. Obesity and physical activity in the daily life of patients with COPD. Lung. 2012; 190: 403-10.

72. Donini LM, Poggiogalle E, Mosca V, Pinto A, Brunani A and Capodaglio P. Disability affects the 6-minute walking distance in obese subjects (BMI>40 kg/ $\left.\mathrm{m}^{2}\right)$. PLoS One. 2013; 8: e75491.

73. Sciurba F, Criner GJ, Lee SM, et al. Six-minute walk distance in chronic obstructive pulmonary disease: reproducibility and effect of walking course layout and length. Am J Resp Crit Care. 2003; 167: 1522-7.

74. Weir NA, Brown AW, Shlobin OA, et al. The influence of alternative instruction on 6-min walk test distance. Chest. 2013; 144: 1900-5.

75. Guyatt GH, Pugsley SO, Sullivan MJ, et al. Effect of encouragement on walking test performance. Thorax. 1984; 39: 818-22.

76. Lord SR and Menz HB. Physiologic, psychologic, and health predictors of 6-minute walk performance in older people. Arch Phys Med Rehabil. 2002; 83: 907-11.

77. Annegarn J, Spruit MA, Savelberg HH, et al. Differences in walking pattern during 6-min walk test between patients with COPD and healthy subjects. PLoS One. 2012; 7: e37329.

78. Smith MD, Chang AT, Seale HE, Walsh JR and Hodges PW. Balance is impaired in people with chronic obstructive pulmonary disease. Gait Posture. 2010; 31: 456-60.

79. Pellegrino R, Viegi G, Brusasco V, et al. Interpretative strategies for lung function tests. Eur Respir J. 2005; 26: 948-68.

80. Jones RL and Nzekwu MM. The effects of body mass index on lung volumes. Chest. 2006; 130: 827-33.

81. Spruit MA, Polkey MI, Celli B, et al. Predicting outcomes from 6-minute walk distance in chronic obstructive pulmonary disease. J Am Med Dir Assoc. 2012; 13: 291-7.

82. Emtner MI, Arnardottir HR, Hallin R, Lindberg E and Janson C. Walking distance is a predictor of exacerbations in patients with chronic obstructive pulmonary disease. Respir Med. 2007; 101: 1037-40.

83. Motegi $T$, Jones RC, Ishii $T$, et al. A comparison of three multidimensional indices of COPD severity as predictors of future exacerbations. Int J Chron Obstruct Pulmon Dis. 2013; 8: 259-71.

84. Enfield K, Gammon S, Floyd J, et al. Six-minute walk distance in patients with severe end-stage COPD: association with survival after inpatient pulmonary rehabilitation. J Cardiopulm Rehabil Prev. 2010; 30: 195-202.

85. Pinto-Plata VM, Cote C, Cabral H, Taylor J and Celli BR. The 6-min walk distance: change over time and value as a predictor of survival in severe COPD. Eur Respir J. 2004; 23: 28-33.

86. Szekely LA, Oelberg DA, Wright C, et al. Preoperative predictors of operative morbidity and mortality in COPD patients undergoing bilateral lung volume reduction surgery. Chest. 1997; 111: 550-8.

87. Cote CG, Pinto-Plata V, Kasprzyk K, Dordelly LJ and Celli BR. The 6-min walk distance, peak oxygen uptake, and mortality in COPD. Chest. 2007; 132: 1778-85.

88. Bowen JB, Votto JJ, Thrall RS, et al. Functional status and survival following pulmonary rehabilitation. Chest. 2000; 118: 697-703.

89. Tertemiz KC, Komus N, Ellidokuz H, Sevinc $\mathrm{C}$ and Cimrin AH. [Mortality and factors affecting mortality in chronic obstructive pulmonary disease]. Tuberk Toraks. 2012; 60: 114-22.

90. de Torres JP, Casanova C, Cote CG, et al. Six-minute walking distance in women with COPD. COPD. 2011; 8: $300-5$.

91. Puhan MA, Garcia-Aymerich J, Frey M, et al. Expansion of the prognostic assessment of patients with chronic obstructive pulmonary disease: the updated BODE index and the ADO index. Lancet. 2009; 374: 704-11.

92. Cote CG, Casanova C, Marin JM, et al. Validation and comparison of reference equations for the 6-min walk distance test. Eur Respir J. 2008; 31: 571-8.

93. Casanova C, Cote CG, Marin JM, et al. The 6-min walking distance: long-term follow up in patients with COPD. Eur Respir J. 2007; 29: 535-40. 


\section{Chapter 8}

94. Ozgür ES, Nayci SA, Ozge C and Tasdelen B. An integrated index combined by dynamic hyperinflation and exercise capacity in the prediction of morbidity and mortality in COPD. Respir Care. 2012; 57: 1452-9.

95. Polkey MI, Spruit MA, Edwards LD, et al. Six-minute-walk test in chronic obstructive pulmonary disease: minimal clinically important difference for death or hospitalization. Am J Respir Crit Care Med. 2013; 187: 382-6.

96. Andrianopoulos V, Wouters EF, Pinto-Plata VM, et al. Prognostic value of variables derived from the sixminute walk test in patients with COPD: Results from the ECLIPSE study. Respir Med. 2015; 109: 1138-46.

97. Troosters T, Gosselink R and Decramer M. Exercise training in COPD: how to distinguish responders from nonresponders. J Cardiopulm Rehabil. 2001; 21: 10-7.

98. Golpe R, Perez-de-Llano LA, Mendez-Marote L and Veres-Racamonde A. Prognostic value of walk distance, work, oxygen saturation, and dyspnea during 6-minute walk test in COPD patients. Respir Care. 2013; 58: 1329-34.

99. Casanova C, Cote C, Marin JM, et al. Distance and oxygen desaturation during the 6-min walk test as predictors of long-term mortality in patients with COPD. Chest. 2008; 134: 746-52.

100. Celli BR, Cote CG, Marin JM, et al. The body-mass index, airflow obstruction, dyspnea, and exercise capacity index in chronic obstructive pulmonary disease. N Engl J Med. 2004; 350: 1005-12.

101. Celli BR, Cote CG, Lareau SC and Meek PM. Predictors of Survival in COPD: more than just the FEV1. Respir Med. 2008; 102 Suppl 1: S27-35.

102. Marin JM, Cote CG, Diaz O, et al. Prognostic assessment in COPD: Health related quality of life and the BODE index. Respir Med. 2011; 105: 916-21.

103. Cote $C$ and Celli B. Effect of exacerbations of COPD (AE) on the multidimensional body mass index (B), airflow obstruction (O), dyspnea (D) and exercise capacity (E), BODE index. Chest. 2004: 840S.

104. Wong R, Sibley KM, Hudani M, et al. Characteristics of people with chronic lung disease who rest during the six-minute walk test. Arch Phys Med Rehabil. 2010; 91: 1765-9.

105. Vogiatzis I and Zakynthinos S. Factors Limiting Exercise Tolerance in Chronic Lung Diseases. Compr Physiol. 2012; 2: 1779-817.

106. Calverley P. Exercise and dyspnoea in COPD. Eur Respir J. 2006; 15: 72-9.

107. Bautmans I, Lambert M and Mets T. The six-minute walk test in community dwelling elderly: Influence of health status. BMC Geriatrics. 2004; 4: 1-9.

108. Flaherty KR, Andrei AC, Murray S, et al. Idiopathic pulmonary fibrosis: prognostic value of changes in physiology and six-minute-walk test. Am J Respir Crit Care Med. 2006; 174: 803-9.

109. Miyamoto S, Nagaya N, Satoh T, et al. Clinical correlates and prognostic significance of six-minute walk test in patients with primary pulmonary hypertension. Comparison with cardiopulmonary exercise testing. Am J Respir Crit Care Med. 2000; 161: 487-92.

110. Roul G, Germain P and Bareiss P. Does the 6-minute walk test predict the prognosis in patients with NYHA class II or III chronic heart failure? Am Heart J. 1998; 136: 449-57.

111. Olsson LG, Swedberg K, Clark AL, Witte KK and Cleland JG. Six minute corridor walk test as an outcome measure for the assessment of treatment in randomized, blinded intervention trials of chronic heart failure: a systematic review. Eur Heart J. 2005; 26: 778-93.

112. Kent BD, Mitchell PD and McNicholas WT. Hypoxemia in patients with COPD: cause, effects, and disease progression. Int J Chron Obstruct Pulmon Dis. 2011; 6: 199-208.

113. Kelley MA, Panettieri RA, Jr. and Krupinski AV. Resting single-breath diffusing capacity as a screening test for exercise-induced hypoxemia. Am J Med. 1986; 80: 807-12.

114. Owens GR, Rogers RM, Pennock BE and Levin D. The diffusing capacity as a predictor of arterial oxygen desaturation during exercise in patients with chronic obstructive pulmonary disease. N Eng/ J Med. 1984; 310: 1218-21.

115. Hadeli KO, Siegel EM, Sherrill DL, Beck KC and Enright PL. Predictors of oxygen desaturation during submaximal exercise in 8,000 patients. Chest. 2001; 120: 88-92.

116. Mahler DA, Gifford AH, Waterman LA, Ward J, Machala S and Baird JC. Mechanism of greater oxygen desaturation during walking compared with cycling in patients with COPD. Chest. 2011; 140: 351-8. 
117. Hussein K, Farouk Alkarn A, Hamdi S, El-Sokkary R and Shams H. Factors predicting exercise-induced oxygen desaturation in stable COPD. Eur Respir J. 2012; 40.

118. Shim SW, Jun YJ, Yong SK, et al. Factors related to exertional oxygen desaturation in patients with COPD. Tuberc Respir Dis. 2011; 70: 498-503.

119. van Gestel AJ, Clarenbach CF, Stowhas AC, et al. Prevalence and prediction of exercise-induced oxygen desaturation in patients with chronic obstructive pulmonary disease. Respiration. 2012; 84: 353-9.

120. Turcotte R, Kiteala L, Marcotte JE and Perrault H. Exercise-induced oxyhemoglobin desaturation and pulmonary diffusing capacity during high-intensity exercise. Eur J Appl Physiol Occup Physiol. 1997; 75: 425-30.

121. Garcia-Talavera I, Jimenez Gonzalez P and Dorta Sanchez R. Exercise-induced oxygen desaturation in chronic obstructive pulmonary disease patients. Arch Bronconeumol. 2015; 51: 481-2.

122. O'Donnell DE, D'Arsigny C, Fitzpatrick M and Webb KA. Exercise hypercapnia in advanced chronic obstructive pulmonary disease: the role of lung hyperinflation. Am J Respir Crit Care Med. 2002; 166: 663-8.

123. Crisafulli E, lattoni A, Venturelli $E$, et al. Predicting walking-induced oxygen desaturations in COPD patients: A statistical model. Respir Care. 2013; 58: 1495-503.

124. de Torres JP, Casanova C, Montejo de Garcini A, Aguirre-Jaime A and Celli BR. Gender and respiratory factors associated with dyspnea in chronic obstructive pulmonary disease. Respir Res. 2007; 8: 18.

125. Taguchi O, Gabazza EC, Yoshida M, et al. CT scores of emphysema and oxygen desaturation during lowgrade exercise in patients with emphysema. Acta Radiol. 2000; 41: 196-7.

126. Dawkins PA, Dowson LJ, Guest PJ and Stockley RA. Predictors of mortality in alpha1-antitrypsin deficiency. Thorax. 2003; 58: 1020-6.

127. Park SS, Janis M, Shim CS and Williams MH, Jr. Relationship of bronchitis and emphysema to altered pulmonary function. Am Rev Respir Dis. 1970; 102: 927-36.

128. Morrison NJ, Abboud RT, Ramadan F, et al. Comparison of single breath carbon monoxide diffusing capacity and pressure-volume curves in detecting emphysema. Am Rev Respir Dis. 1989; 139: 1179-87.

129. Turato G, Zuin R, Miniati M, et al. Airway inflammation in severe chronic obstructive pulmonary disease: relationship with lung function and radiologic emphysema. Am J Respir Crit Care Med. 2002; 166: 105-10.

130. Baldi S, Miniati M, Calogero RB, et al. Relationship between Extent of Pulmonary Emphysema by Highresolution Computed Tomography and Lung Elastic Recoil in Patients with Chronic Obstructive Pulmonary Disease. Am J Respir Crit Care Med. 2001; 164: 585-9.

131. Waatevik SE, Bakke P, Eagan T, et al. Diffusion capacity and CT measures of emphysema and airway wall thickness - Relation to arterial oxygen tension in COPD patients. Eur Respir J. 2015; 46.

132. Barbera JA, Ramirez J, Roca J, Wagner PD, Sanchez-Lloret J and Rodriguez-Roisin R. Lung structure and gas exchange in mild chronic obstructive pulmonary disease. Am Rev Respir Dis. 1990; 141: 895-901.

133. Sandek K, Bratel T, Hellstrom G and Lagerstrand L. Ventilation-perfusion inequality and carbon dioxide sensitivity in hypoxaemic chronic obstructive pulmonary disease (COPD) and effects of 6 months of longterm oxygen treatment (LTOT). Clin Physiol. 2001; 21: 584-93.

134. Andrianopoulos V, Celli B, Franssen FM, et al. Determinants of exercise-induced oxygen desaturation including pulmonary emphysema in COPD: Results from the ECLIPSE study. Respir Med. 2016; 119:87-95.

135. Vonk-Noordegraaf A. The shrinking heart in chronic obstructive pulmonary disease. N Eng/ J Med. 2010; 362: 267-8.

136. Barr RG, Bluemke DA, Ahmed FS, et al. Percent emphysema, airflow obstruction, and impaired left ventricular filling. N Engl J Med. 2010; 362: 217-27.

137. Chou KC, Chang SC, Chang HI and Shiao GM. Body position, membrane diffusing capacity and pulmonary capillary blood volume in chronic bronchitis and pulmonary emphysema. Zhonghua Yi Xue Za Zhi. 1999; 62: 209-16.

138. McLean A, Warren PM, Gillooly M, MacNee W and Lamb D. Microscopic and macroscopic measurements of emphysema: relation to carbon monoxide gas transfer. Thorax. 1992; 47: 144-9.

139. Tylen U, Boijsen M, Ekberg-Jansson A, Bake B and Lofdahl CG. Emphysematous lesions and lung function in healthy smokers 60 years of age. Respir Med. 2000; 94: 38-43. 


\section{Chapter 8}

140. Kinsella M, Muller NL, Abboud RT, Morrison NJ and DyBuncio A. Quantitation of emphysema by computed tomography using a "density mask" program and correlation with pulmonary function tests. Chest. 1990; 97: 315-21.

141. Lee JS, Ra SW, Chae EJ, et al. Validation of the lower limit of normal diffusing capacity for detecting emphysema. Respiration. 2011; 81: 287-93.

142. Vogiatzis I, Zakynthinos $G$ and Andrianopoulos V. Mechanisms of physical activity limitation in chronic lung diseases. Pulm Med. 2012; 2012: 634761.

143. Salome CM, King GG and Berend N. Physiology of obesity and effects on lung function. J App/ Physiol (1985). 2010; 108: 206-11.

144. Ray CS, Sue DY, Bray G, Hansen JE and Wasserman K. Effects of obesity on respiratory function. Am Rev Respir Dis. 1983; 128: 501-6.

145. Ora J, Laveneziana P, Ofir D, Deesomchok A, Webb KA and O'Donnell DE. Combined effects of obesity and chronic obstructive pulmonary disease on dyspnea and exercise tolerance. Am J Respir Crit Care Med. 2009; 180: 964-71.

146. Gupta SS, Gothi D, Narula G and Sircar J. Correlation of BMI and oxygen saturation in stable COPD in Northern India. Lung India. 2014; 31: 29-34.

147. Jubran A. Pulse oximetry. Crit Care. 2015; 19: 272.

148. Collins JA, Rudenski A, Gibson J, Howard L and O'Driscoll R. Relating oxygen partial pressure, saturation and content: the haemoglobin-oxygen dissociation curve. Breathe. 2015; 11: 194-201.

149. Niederman MS, Clemente PH, Fein AM, et al. Benefits of a multidisciplinary pulmonary rehabilitation program. Improvements are independent of lung function. Chest. 1991; 99: 798-804.

150. Knower MT, Dunagan DP, Adair NE and Chin R, Jr. Baseline oxygen saturation predicts exercise desaturation below prescription threshold in patients with chronic obstructive pulmonary disease. Arch Intern Med. 2001; 161: 732-6.

151. Andrianopoulos V, Franssen FM, Peeters JP, et al. Exercise-induced oxygen desaturation in COPD patients without resting hypoxemia. Respir Physiol Neurobiol. 2014; 190: 40-6.

152. Garcia-Talavera I, Tauroni A, Trujillo JL, et al. Time to desaturation less than one minute predicts the need for long-term home oxygen therapy. Respir Care. 2011; 56: 1812-7.

153. Stoller JK, Panos RJ, Krachman S, Doherty DE and Make B. Oxygen therapy for patients with COPD: current evidence and the long-term oxygen treatment trial. Chest. 2010; 138: 179-87.

154. Calverley PM. Supplementary oxygen therapy in COPD: is it really useful? Thorax. 2000; 55: 537-8.

155. O'Donnell DE, D'Arsigny $C$ and Webb KA. Effects of hyperoxia on ventilatory limitation during exercise in advanced chronic obstructive pulmonary disease. Am J Respir Crit Care Med. 2001; 163: 892-8.

156. Garcia-Talavera I and Figueira-Goncalves JM. Ambulatory oxygen therapy in COPD patients with oxygen desaturation during exercise. COPD. 2016; 1: 1-3.

157. Croxton TL and Bailey WC. Long-term oxygen treatment in chronic obstructive pulmonary disease: recommendations for future research: an NHLBI workshop report. Am J Respir Crit Care Med. 2006; 174: 373-8.

158. Troosters T. Oxygen: the good, the bad, and the necessary. Thorax. 2004; 59: 1005-6.

159. Kawakami Y, Terai T, Yamamoto $\mathrm{H}$ and Murao M. Exercise and oxygen inhalation in relation to prognosis of chronic obstructive pulmonary disease. Chest. 1982; 81: 182-8.

160. Belfer $\mathrm{MH}$ and Reardon JZ. Improving exercise tolerance and quality of life in patients with chronic obstructive pulmonary disease. J Am Osteopath Assoc. 2009; 109: 268-78

161. Jensen LA, Onyskiw JE and Prasad NG. Meta-analysis of arterial oxygen saturation monitoring by pulse oximetry in adults. Heart Lung. 1998; 27: 387-408.

162. Wouters PF, Gehring H, Meyfroidt G, et al. Accuracy of pulse oximeters: the European multi-center trial. Anesth Analg. 2002; 94: S13-6.

163. Webb RK, Ralston AC and Runciman WB. Potential errors in pulse oximetry. II. Effects of changes in saturation and signal quality. Anaesthesia. 1991; 46: 207-12.

164. Van de Louw A, Cracco C, Cerf C, et al. Accuracy of pulse oximetry in the intensive care unit. Intensive Care Med. 2001; 27: 1606-13. 
165. Vold ML, Aasebo U, Wilsgaard T and Melbye H. Low oxygen saturation and mortality in an adult cohort: the Tromso study. BMC Pulm Med. 2015; 15: 9.

166. Ardestani ME and Abbaszadeh M. The association between forced expiratory volume in one second $\left(\mathrm{FEV}_{1}\right)$ and pulse oximetric measurements of arterial oxygen saturation $\left(\mathrm{SpO}_{2}\right)$ in the patients with COPD: A preliminary study. J Res Med Sci. 2014; 19: 257-61.

167. Deesomchok A, Webb KA, Forkert L, et al. Lung hyperinflation and its reversibility in patients with airway obstruction of varying severity. COPD. 2010; 7: 428-37.

168. Zafar MA, Tsuang W, Lach L, Eschenbacher W and Panos RJ. Dynamic hyperinflation correlates with exertional oxygen desaturation in patients with Chronic Obstructive Pulmonary Disease. Lung. 2013.

169. Harms CA, McClaran SR, Nickele GA, Pegelow DF, Nelson WB and Dempsey JA. Exercise-induced arterial hypoxaemia in healthy young women. J Physiol. 1998; 507: 619-28.

170. Hardie JA, Vollmer WM, Buist AS, Ellingsen I and Morkve O. Reference values for arterial blood gases in the elderly. Chest. 2004; 125: 2053-60.

171. Domingo C, Canturri E, Lujan M, Moreno A, Espuelas H and Marin A. [Transcutaneous measurement of partial pressure of carbon dioxide and oxygen saturation: validation of the SenTec monitor]. Arch Bronconeumol. 2006; 42: 246-51.

172. Stege G, van den Elshout FJ, Heijdra YF, van de Ven MJ, Dekhuijzen PN and Vos PJ. Accuracy of transcutaneous carbon dioxide tension measurements during cardiopulmonary exercise testing. Respiration. 2009; 78: 147-53.

173. Nicolini A and Ferrari MB. Evaluation of a transcutaneous carbon dioxide monitor in patients with acute respiratory failure. Ann Thorac Med. 2011; 6: 217-20.

174. Chhajed PN, Miedinger D, Baty F, et al. Comparison of combined oximetry and cutaneous capnography using a digital sensor with arterial blood gas analysis. Scand J Clin Lab Invest. 2010; 70: 60-4.

175. Andrianopoulos V, Vanfleteren LE, Jarosch I, et al. Transcutaneous carbon-dioxide partial pressure trends during six-minute walk test in patients with very severe COPD. Respir Physiol Neurobiol. 2016; 233: 52-9.

176. Whitesell R, Asiddao C, Gollman D and Jablonski J. Relationship between arterial and peak expired carbon dioxide pressure during anesthesia and factors influencing the difference. Anesth Analg. 1981; 60: 508-12.

177. Javadpour SM, Selvadurai H, Wilkes DL, Schneiderman-Walker J and Coates AL. Does carbon dioxide retention during exercise predict a more rapid decline in $\mathrm{FEV}_{1}$ in cystic fibrosis? Arch Dis Child. 2005; 90 : 792-5.

178. Montes de Oca M and Celli BR. Respiratory muscle recruitment and exercise performance in eucapnic and hypercapnic severe chronic obstructive pulmonary disease. Am J Respir Crit Care Med. 2000; 161: 880-5.

179. Pellegrino R and Brusasco V. Lung hyperinflation and flow limitation in chronic airway obstruction. Eur Respir J. 1997; 10: 543-9.

180. Puente-Maestu L and Stringer WW. Hyperinflation and its management in COPD. Int J Chron Obstruct Pulmon Dis. 2006; 1: 381-400.

181. O'Donnell DE, Revill S and Webb K. Dynamic hyperinflation and exercise intolerance in COPD. Am J Respir Crit Care Med. 2001; 164: 770-7.

182. O'Donnell DE and Parker CM. COPD exacerbations. 3: Pathophysiology. Thorax. 2006; 61: 354-61.

183. Bloch-Salisbury E, Shea SA, Brown R, Evans K and Banzett RB. Air hunger induced by acute increase in $\mathrm{PCO}_{2}$ adapts to chronic elevation of $\mathrm{PCO}_{2}$ in ventilated humans. J App/ Physiol (1985). 1996; 81: 949-56.

184. Marini JJ and Roussos C. Round table conference on ventilatory failure, Brussels, Belgium, March 16-18, 1991. Intensive Care Med. 1992; 18: 190-2.

185. Begin P and Grassino A. Inspiratory muscle dysfunction and chronic hypercapnia in chronic obstructive pulmonary disease. Am Rev Respir Dis. 1991; 143: 905-12.

186. Sorli J, Grassino A, Lorange $G$ and Milic-Emili J. Control of breathing in patients with chronic obstructive lung disease. Clin Sci Mol Med. 1978; 54: 295-304.

187. Gorini M, Misuri G, Corrado A, et al. Breathing pattern and carbon dioxide retention in severe chronic obstructive pulmonary disease. Thorax. 1996; 51: 677-83. 


\section{Chapter 8}

188. Roussos C and Koutsoukou A. Respiratory failure. Eur Respir J Suppl. 2003; 47: 3s-14s.

189. Younes M. Determinants of thoracic excursion during exercise. New York: Marcel Dekker, 1991.

190. Light R, Mahutte $\mathrm{C}$ and Brown S. Etiology of carbon dioxide retention at rest and during exercise in chronic airflow obstruction. Chest. 1988; 94: 61-7.

191. Laviolette L, Sava F, O'Donnell DE, et al. Effect of obesity on constant workrate exercise in hyperinflated men with COPD. BMC Pulm Med. 2010; 10: 33.

192. Franssen FM, O'Donnell DE, Goossens GH, Blaak EE and Schols AM. Obesity and the lung: 5. Obesity and COPD. Thorax. 2008; 63: 1110-7.

193. O'Donnell DE, Deesomchok A, Lam YM, et al. Effects of BMI on static lung volumes in patients with airway obstruction. Chest. 2011; 140: 461-8.

194. Vishnu SM, Anupama N, Harsha DS, et al. Low body mass index and low serum albumin level as risk factors for carbon dioxide retention during acute exacerbation of chronic obstructive pulmonary disease. Int. j. innov. res. sci. eng. technol 2013; 2: 3508.

195. Aitken ML, Franklin JL, Pierson DJ and Schoene RB. Influence of body size and gender on control of ventilation. J Appl Physiol (1985). 1986; 60: 1894-9.

196. Narkiewicz K, Kato M, Pesek CA and Somers VK. Human obesity is characterized by a selective potentiation of central chemoreflex sensitivity. Hypertension. 1999; 33: 1153-8.

197. Ora J, Laveneziana P, Ofir D, Deesomchok A, Webb KA and O'Donnell DE. Combined effects of obesity and COPD on dyspnea and exercise tolerance. Am J Respir Crit Care Med. 2009; 15: 180: 964-71

198. Wimberley PD, Pedersen KG, Thode J, Fogh-Andersen N, Sorensen AM and Siggaard-Andersen O. Transcutaneous and capillary $\mathrm{pCO}_{2}$ and $\mathrm{pO}_{2}$ measurements in healthy adults. Clin Chem. 1983; 29: 1471-3.

199. West JB. Causes of and compensations for hypoxemia and hypercapnia. Compr Physiol. 2011; 1: 1541-53.

200. Sedgwick P. Retrospective cohort studies: advantages and disadvantages. BMJ. 2014; 348: g1072.

201. Fiore C, Lee A, McDonald C, Hill C and Holland A. Should oxyhaemoglobin saturation be monitored continuously during the 6-minute walk test? Chron Respir Dis. 2011; 8: 181-4. 
Summary 

Chronic obstructive pulmonary disease (COPD) is a progressive lung disease characterized by persistent airflow limitation and impaired exercise capacity. Chronic bronchitis and pulmonary emphysema are the two main conditions included in COPD. Patients with COPD demonstrate a generally impaired health status and have increase rates of morbidity and mortality. Dyspnea is the cardinal symptom in COPD, which results in impaired exercise capacity and has a negative impact on daily life activities and quality of life. The perception of dyspnea is exaggerated in those patients during physical activity or exercise due to reduced breathing efficiency that results from the deteriorating ventilatory mechanics and the increased ventilatory requirement. Moreover, pathophysiological effects of cardiovascular and peripheral muscle abnormalities can further explain and intensify dyspnea as well as increased muscle fatigue in COPD. As a consequence, exercise capacity in those patients is symptomlimited and determined by a complex interaction of several pathophysiological factors in COPD. Therefore, exercise capacity can reflect the impact of disease severity on patients with COPD and can provide clinicians and health professionals with important clinical information.

Clinical exercise testing has become an important clinical tool to assess and evaluate exercise capacity and predict outcomes in patients with COPD. Therefore, this thesis focused on field exercise testing and specifically on the six-minute walk test (6MWT) aiming at extending the clinical information derived from the 6MWT and at increasing test utilization in clinical practice. After describing the overall burden of COPD disease and its negative impact on exercise capacity in patients, Chapter 1 introduced the field tests and demonstrated the clinical and prognostic value of the 6MWT as reliable clinical instrument according to the recent evidences in literature. Indeed, 6MWT has been shown to provide useful information for the impact of disease severity in patients' functional status, the effectiveness of therapeutic interventions and can predict mortality and hospitalization. The main outcome of the 6MWT is the six-minute walk distance (6MWD) which represents exercise capacity and can change after therapeutic intervention or across the progress of disease severity influencing the clinical decisionmaking process.

Several determinants of exercise performance can affect 6MWD. Chapter 2 aimed at investigating the characteristics of functional exercise performance and determinants of the cycle endurance test (CET) and 6MWT in a large clinical cohort of COPD patients. A retrospective analysis in a large dataset of 2050 patients demonstrated different determinants of exercise performance between CET and 6MWT. The main findings were that the overall perception of dyspnea and sex-related differences determine the CET- $T_{\text {end }}$ but BMI, FEV 1 and FRC influence the 6MWD. Chapter 2 suggested that CET and $6 \mathrm{MWT}$ are not interchangeable exercise tests; gender difference should temper the interpretation of CET and that even though the fixed proportion of workload capacity does not provide the same physiological stress among patients, improvement in CET- 
$T_{\text {end }}$ after a therapeutic intervention such as a pulmonary rehabilitation (PR) program are responsive of improvements in clinical status of patients with COPD.

Several reference equations for the 6MWT have been formulated to facilitate the interpretation of the walked 6MWD and help to estimate the degree of exercise tolerance in patients. However, applying different reference equations could result in different 6MWD values of predicted leading to misinterpretation of the levels of exercise capacity or improvements of physical performance after therapeutic interventions. Therefore, Chapter 3 investigated the impact of several 6MWD reference equations and factors accountable for different 6MWD \%predicted values in a large dataset of 2757 patients with COPD. Chapter 3 reported that existing 6MWD reference equations will give varying results and highlighted the importance of the selection of an appropriate reference equation. It suggested that the choice of 6MWD reference equation should consider the consistency 6MWT operating procedures and at least be specific for the country/region of origin.

Besides the interpretation of 6MWD, the estimation of prognosis according to walking performance is clinically important. In addition to the 6MWD, other 6MWT derived variables, such as mean walk-speed (6MWSpeed), 6-min walk-work (6MWW), distance-saturation product (DSP), exercise-induced oxygen desaturation (EID), and unintended stops seems to be useful for the prediction of mortality and hospitalization in COPD. Chapter 4, explored the association between 6MWT-derived variables and mortality as well as hospitalization and compared it with the BODE index using the ECLIPSE cohorts in a three-year follow-up. Cox's proportional-hazard regressions were performed to estimate 3-year mortality and hospitalization and demonstrated that the $6 \mathrm{MWT}$-derived variables have an additional predictive value of mortality in patients with COPD. Chapter 4, indicated the DSP as the strongest predictor of mortality and the BODE index as the most sensitive tool for assessing the risk of hospitalization while suggested that unintended stop(s) during the 6MWT can refine the prognosis of mortality in COPD.

Impaired gas exchange can produce varying degrees of $V^{\prime} / Q^{\prime}$ inequalities, diffusion impairment, and hypoxemia during exercise. Chapter 5, aimed at investigating the prevalence of exercise-induced desaturation (EID), the relative-weight of several physiological determinants of EID including pulmonary emphysema, and the relationship of EID with certain patients' clinical characteristics using a dataset of 2050 COPD patients from the ECLIPSE cohort. Chapter 5 demonstrated that $21 \%$ of patients exhibited EID in the ECLIPSE cohort and showed that advanced emphysema, obesity, severe airflow limitation, and low resting oxygen saturation are associated with EID. Chapter 5 also suggested that emphysematous patients with high ADO-score should be monitored for EID.

In clinical practice, several screening tests have been proposed to predict EID, including $\mathrm{FEV}_{1}$, DLCO and baseline-SpO . Chapter 6 aimed to validate a proposed cut-off 
of baseline- $\mathrm{SpO}_{2} \leq 95 \%$ as simple screening procedure to predict EID during 6MWT and investigated the prevalence and characteristics of patients exhibited EID to $\mathrm{SpO}_{2}$ nadir $\leq 88 \%$ using 402 non-hypoxemic COPD patients referred for pulmonary rehabilitation (PR). Chapter 6 reported that 39\% of those patients, who were referred for PR, exhibited EID and that those patients may be suitable candidates for ambulatory oxygen therapy. Chapter 6 did not corroborate baseline $\mathrm{SpO}_{2} \leq 88 \%$ as highly accurate in predicting EID, and thus concluded that baseline $\mathrm{SpO}_{2}$ solely is not adequate to predict EID. In contrast, a combination of several clinical characteristics including DLCO, FEV $\mathrm{PaO}_{2}$, baseline- $\mathrm{SpO}_{2}$, and sex, increases substantially the odds for EID and can facilitate the prediction of EID in COPD.

Besides the EID, the variable of carbon-dioxide partial pressure $\left(\mathrm{PCO}_{2}\right)$ has been also reported as prognostic factor in COPD. Chapter 7, aimed to define patterns of transcutaneous carbon-dioxide partial-pressure $\left(\mathrm{T}_{\mathrm{C}} \mathrm{PCO}_{2}\right)$ trends during 6MWT and to study determinants of $\mathrm{CO}_{2}$-retention and exercise-induced hypercapnia (EIH) using a group of 62 patients with very severe COPD. Chapter 7 demonstrated that $50 \%$ of the patients with COPD exhibited $\mathrm{CO}_{2}$-retention, $26 \%$ preserved and $24 \%$ reduced $\mathrm{T}_{\mathrm{C}} \mathrm{PCO}_{2}$ levels while in all group 31\% patients presented ElH during the 6MWT. Chapter 7 also showed that even though $\mathrm{PCO}_{2}$ response to 6MWT is highly heterogeneous, a very low $\mathrm{FEV}_{1}$ and increased baseline- $\mathrm{P}_{\mathrm{C}} \mathrm{CO}_{2}$ together with pre-walk dyspnea increase the risk for $\mathrm{CO}_{2}$-retention and $\mathrm{EIH}$. Moreover, chapter 7 revealed that an overweight BMI seems to carry a protective effect against EIH in very severe COPD. 

Valorization 

Valorization is the process by which scientific knowledge can shape and influence the world and have practical application. Knowledge valorization specifically refers to "getting the maximum value and usefulness out of education programs and managed projects, by generalizing what has been learnt from the specialist experiences to other, related fields".

Based on this definition, this chapter will discuss the current findings of this thesis in the light of the social and economic impact as well as innovative concepts for future health care.

\section{RELEVANCE AND INNOVATION}

COPD is highly prevalent disease and a leading cause of morbidity and mortality worldwide. Besides the impairment of pulmonary function, several other factors influence the impact and the evolution of COPD. These factors include the occurrence of hypoxemia or hypercapnia at exertion, limiting functional dyspnea and diminished exercise capacity that are associated with higher risk of mortality and hospitalization. Indeed, the degree of exercise tolerance and pathophysiological response to exercise facilitate the prognosis and can constitute an essential dimension in the evaluation of COPD patients. Clear exercise outcomes and their appropriate interpretation can play a superior role in COPD management and treatment in clinical practice.

This thesis provided new evidence in this field of expertise:

In chapter 2, a comparison between two popular tests of exercise assessment (CET and the 6MWT) in clinical practice revealed different determinants of exercise capacity between CET and the 6MWT. According to this, CET and 6MWT are not interchangeable. Patients' clinical characteristics should be considered in the interpretation of exercise outcomes derived from CET or 6MWT. Indeed, the new knowledge reported in this thesis facilitates the choice of exercise test and the interpretation of the exercise outcomes which have become an integral part of the evaluation and response to treatment in COPD.

Chapter 3 addresses an important concern about the choice of the appropriate $6 \mathrm{MWT}$ reference equation. The right interpretation of walking performance according to the estimation and inference for normative values is great of importance for both health professionals and patients. For health professionals, the estimation of walking capacity using reference values can represent the severity of patients' exercise intolerance and thus influence on the clinical decision. In addition, reference values are critical to tailor appropriately timed exercise interventions indicating the high importance of choosing the right reference equation. For the patients, walking capacity 
expressed as percentage of predicted, can be used to increase the awareness about the impact of disease severity on their exercise capacity.

Chapter 4 investigated the prognostic value of existing and new variables derived from the six-minute walk test in patients with COPD. This knowledge can increase the utilization of the 6MWT in praxis and provide health professionals with insight for the prognostic value of each 6MWT-derived variable. Moreover, the demonstrated prognostic value of the 6MWT-derived variable can also be considered in the clinical decision-making process.

In chapter 5, the relationship of EID with certain patients' clinical characteristics including pulmonary emphysema and the relative-weight of these determinants on the occurrence of EID was demonstrated. This knowledge provides health professionals with a better understanding about the pathophysiological interaction of several determinants of EID and the risk factors for the occurrence of EID in COPD patients with different degrees of pulmonary emphysema. Practically, health professionals could partly estimate the risks for the occurrence of EID during the 6MWT based on patients' two basic characteristics which are the degree of pulmonary emphysema and lung obstruction severity according to the data presented in this chapter.

Chapter 6 reported that baseline oxygen saturation levels solely are inaccurate to predict EID, while it demonstrated a combination of clinical characteristics that increases the odds for EID in COPD. Indeed, this is practical knowledge for health professionals who could expect based on certain patients' characteristics the occurrence of EID prior to exercise testing. Also, it can provide health professionals with rapid clinical information about the risk of EID in cases that the application of a 6MWT is not instantly possible.

Chapter 7 demonstrated the high heterogeneity of $\mathrm{PCO}_{2}$ responses during the $6 \mathrm{MWT}$ and the determinants of $\mathrm{CO}_{2}$ retention and $\mathrm{EIH}$ in COPD patients. Indeed, $\mathrm{PCO}_{2}$ response during the 6MWT may represent a marker of disease severity that is beyond the walking capacity. Practically, health professionals could assess $\mathrm{PCO}_{2}$ trends during the 6MWT and specifically the degree of $\mathrm{CO}_{2}$ retention during walking for the evaluation of therapeutic interventions such as inspiratory muscle training (MIT) or consider the efficiency of non-invasive ventilation in COPD. Moreover, the findings of this chapter could suggest the transcutaneous monitoring of $\mathrm{CO}_{2}$ during $6 \mathrm{MWT}$ for the identification of patients who need special attention regarding to $\mathrm{EIH}$. 


\section{TARGET GROUPS}

\section{Health care professionals}

The main target group of this thesis is health-care professionals including mostly physicians, exercise physiologists, physiotherapists, and nurses. The findings of this thesis provided further insight of the widely-used field test of the 6MWT and the COPD pathophysiological responses to exercise. These findings emphasize the importance of exercise assessment in clinical practice and help to increase the utilization of the 6MWT and the interpretation as well as the prognostic value of the 6MWT-derived variables. Health care professionals are encouraged to assess exercise capacity of the patients and consider exercise outcomes in the clinical decision-making process. Exercise outcomes increase the clinical information of patients' health status, support timely treatment and facilitate the prognosis.

\section{Patients with COPD}

It is a consensus that patient is the most important member of the interdisciplinary health care team. Patients can benefit of the output of this thesis increasing their awareness of the disease, understanding the significance and the role of field exercise testing in clinical practice, and realizing the clinical importance of assessing exercise capacity. Indeed, patients who are aware about their disease and their clinical condition are more likely to have better outcomes. In contrast, patients with low levels of awareness are likely to have poor motivation and being not adequately cooperative in therapeutic plans and intervention. The consequences can include low therapeutic efficacy and added medical expenses. Therefore, the knowledge including in this thesis can be used by COPD patients for increasing their awareness about the disease and utilizing actions plans more effectively, which can result in obtaining the most benefits from the therapeutic procedure.

\section{ACTIVITIES AND PRODUCTS}

The findings of the studies in this thesis have resulted in numerous scientific abstracts on international congresses all over the world. All the studies have been published in recognized scientific journals in the respiratory field and they have received numerous citations. Data from chapter 2 have been published in the official European Respiratory Society (ERS) statement of the use of exercise testing in the evaluation of interventional efficacy (Puente-Maestu L. et al. Eur Respir J 2016). Moreover, some abstracts of the studies included in this thesis were awarded with travel grants in order to be presented in international congresses in Europe and USA and be the topic of discussions amongst 
health-professionals. Considering this publicity, the findings of this study have been widely distributed and may constitute an inspiration for future research questions. Indeed, relative future research is essential as exercise testing can be the cornerstone of chronic patients' clinical evaluation. 
Acknowledgements 

The ancient Greek philosopher Aristotle had told "All human actions have one or more of these seven causes: chance, nature, compulsion, habit, reason, passion and desire". Writing this note of thanks as the finishing touch of my thesis, I wish to thanks all the people who were behind on my personal causes that making me successfully complete this PhD thesis.

First of all, I would like to express my sincere gratitude to my supervisors Professor Wouters and Professor Spruit (or Martijn, as he always liked to be addressed by his first name). Professor Wouters thank you for being an inspiration for me and for giving me motivation throughout the intense period of my PhD. Your immense knowledge and your guidance were more than valuable. Honestly, I have great respect for you and I really appreciate your support and kindness. Dear Martijn, I want to thank you for all the opportunities I was given to conduct my research during the $\mathrm{PhD}$ and for your important advices and instruction for writing good scientific articles and completing my thesis. I consider myself lucky that I learned how to write scientific articles from one of the best on it. Also, I appreciate the fact that you helped me to increase my scientific network introducing me to the best scientists on the respiratory field. Dank u wel!

Dear Professor Vogiatzis, the words are really not enough to express my gratitude to you. My whole research career started from you who gave me the opportunity and trust me to be a member of your research team in Athens. I learned how to do research from you who are one of the most capable and recognizable professors on conducting clinical experiments with patients. Undoubtedly, you are my greatest mentor in research. Your positive influence and your support as co-supervisor provided me with the attributes to complete my thesis. Euxapıбtú!

Dear Professor Klaus Kenn, thank you for the cooperation regarding one of the studies included in my thesis and your kindness. I am also deeply thankful for the post-PhD chance you gave me agreeing to apply together for the Marie Sklodowska-Curie fellowship and your warm welcome at the Schön klinik in Berchtesgaden! I want also to thank my German colleagues Rainer, Inga, and Tessa for their assistance (especially in the German language!) and teamwork. Danke Schön!

I want also to acknowledge my degree- and assessment- committee members: Prof. G.J. Wesseling, Prof. H.P. Brunner-La Rocca, Prof. Y.F. Heijdra, Prof. J.P. Kooman, Prof. E. Derom, Prof. K. Kenn and Prof. N.G. Koulouris who graciously agreed to serve as members on my PhD committee. I appreciate it! Thank you very much!

I need also to express my sincere appreciation to Ingrid Augustin for her valuable assistance during my PhD period. I am also very thankful to Gerrie Janssen for her help and guidance in organizational issues related to my PhD. Many thanks also go to the great physicians of $\mathrm{ClRO}+$, Dr. Frits Franssen, Dr. Lowie Vanfleteren and Dr. Janssen Daisy for their valuable medical opinion and advices regarding findings of this thesis. 
I also appreciate the help and advices of Groenen Miriam in statistics and I also want to say a thank you to Dr. Maurice Sillen for his kindness.

I would also like to thank my $\mathrm{CIRO}+$ colleagues with whom I shared the same room during my PhD for their cooperation and friendliness. Dear Rafael, Sarah, Dionne B., Dionne S., Jeannet, Fionna, Carmen, Nienke, Wai-Yan, Anouk, Esther, Yvonne, Cindy and Coby thank you very much for your support and the nice and friendly atmosphere!

I owe my thanks to all of my co-authors in the studies of this thesis, who helped me to improve the manuscripts with their useful and constructive comments and suggestions. In regards to the ECLIPSE studies included in thesis, special thanks go to Professors Alvar Agusti, Jørgen Vestbo, Bartolome Celli, Peter Calverley, Victor Pinto-Plata, Per Bakke, Stephen Rennard, William MacNee, and to Julie Yates and Ruth Tal-Singer for the critical comments and suggestions.

I would like also to acknowledge the European Respiratory Society (ERS) for the tremendous positive impact on my research career. In 2012, I received an ERS LongTerm Research Fellowship (LTRF 63-2012) that offered me the chance to move from THORAX research center in Athens to CIRO in the Netherlands. This LTRF created the great opportunity to continue at CIRO+ for a PhD. Recently (2016) I was the recipient of an ERS-EU RESPIRE2 Marie Sklodowska-Curie Postdoctoral Research Fellowship Number MCF (8465)-2015 that allowed me to move from CIRO+ in the Netherlands to the Schön klinik Berchtesgadener Land in Germany for a post-PhD fellowship.

I want to thank Professor Robert Bals for all the given support in the beginning of my ERS LTRF when I moved at CIRO+ center where, afterwards, I continued with the PhD course. Also, I truly appreciate his involvement in Marie Sklodowska-Curie Fellowship as my external mentor. Dear Robert, thank you very much!

I am also deeply thankful to the Director of the THORAX Research Foundation of Intensive and emergency Medicine Professor Charalambos Roussos and to Captain Vassilis Konstantakopoulos (Captain Vassilis \& Carmen Foundation) for their valuable support to the first steps of my career in research. Although they were not directly involved in my PhD, they laid the foundation stone for starting and developing my career in research. I really thank you!

A special word of thank I would like to address to Kerstin Fritz for her invaluable support to my life abroad throughout my PhD period. Kerstin, I thank you very much for your motivation, your help and all the precious moments. You are really behind of many personal accomplishments and achievements I did these years. Herzlichen Dank!

Many thanks also go to my friend and colleague Zafeiris and Erica, my Paranymphs in PhD defense. Zafeiris, we started doing our first steps in research having a very good

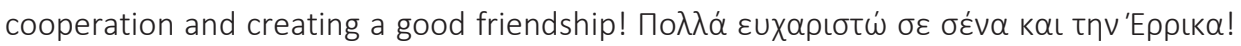


Also, I need to thank my close Greek friends Alexandros and Spiros as well as my Brazilian friend Rodolfo for their support and friendliness during my PhD period.

Last but not least, my family deserves special thanks for supporting me over the years and for encouraging me to pursue an advance degree. Without my parents Eleni, Dimitris and my sister Marianna, I may never have gotten to where I am today.

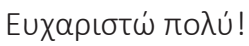




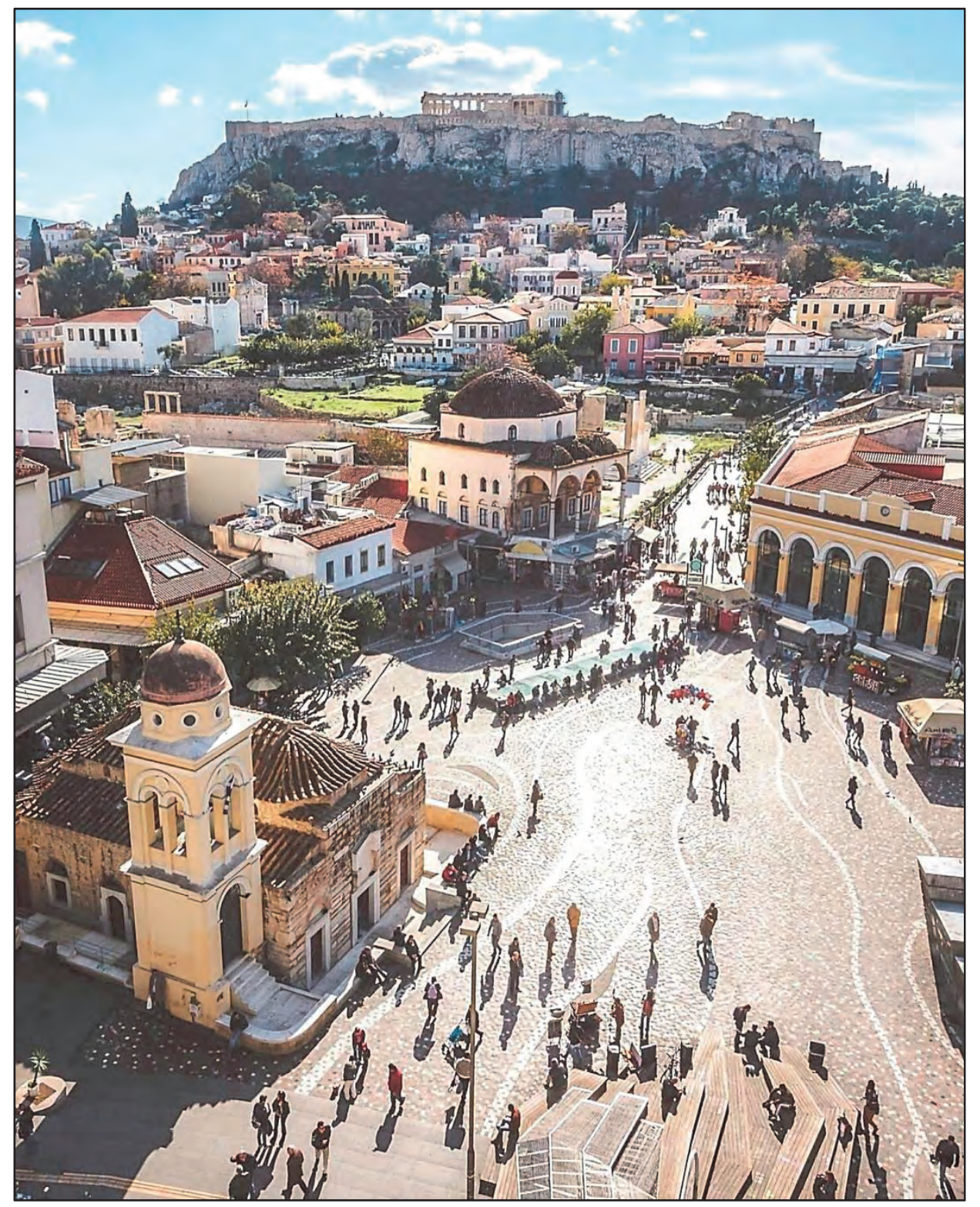

I would also like to acknowledge my home city Athens. Short trips to Athens during the intensive period of PhD course always helped me to rest and relax so that I could feel energetic again 
Curriculum vitae 

Vasileios Andrianopoulos (VA) was born on $28^{\text {th }}$ January 1980 in Athens (Greece). In 2003, VA obtained his Bachelor Degree in "Physical Education and Sports Science" from the University of Thessaly, Greece. During his bachelor study, VA was selected by Professor loannis Koutentakis to join the University's research team; thereby he had the opportunity to write a bachelor thesis entitled "Smoking and risk factors for coronary disease in a Greek population over 50 years old". In 2010, VA completed a master degree with excellent mark in "Exercise and Health" at the University of Thessaly. His thesis was entitled "The effect of expiratory flow limitation on oxygen kinetic of peripheral muscles" and the research measurements were performed at Thorax Foundation in Athens (Greece) under the supervision of Professor loannis Vogiatzis.

VA worked for almost 5 years as young researcher at Thorax foundation in Athens, a recognized research center of Intensive and emergency Medicine directed by Professor Dr. Charalambos Roussos. Within the frames of his work at Thorax foundation, he also worked for the large European Research project "PROACTIVE"-items of PROactive tools for measuring physical activity in COPD. Additionally, VA offered his services in the Pulmonary Rehabilitation of the $1^{\text {st }}$ University clinic at "Sotiria" hospital in Athens and he implemented numerous ergospirometric measurements for COPD and CHF patients referred to Pulmonary Rehabilitation. In 2012, VA obtained a European Respiratory Society (ERS) Long Term Research Fellowship (LTRF 63-2012) and moved from the Thorax foundation in Athens (Greece) to CIRO+ Center of expertise for chronic organ failure in Horn (the Netherlands). VA completed his LTRF performing research analyses on large databases of patients with COPD and afterwards continued at CIRO+ center for a PhD course. In 2016, VA was the recipient of an ERS-EU RESPIRE2 Marie Sklodowska-Curie Post-doctoral Research Fellowship and moved from $\mathrm{CIRO}+$ to Schön Klinik in Berchtesgadener Land (Germany).

VA is an active member of the "ERS College of Experts" and has participated in the ATS/ERS Task Force for the development of Health Technology Assessment for patients' field testing. He has expertise in the operation of several clinical devices (NIRO [Hamamatsu], PORTALITE [Artinis], Physioflow [Manatec], SenTec [Sentec AG], OptoElectronic Plethysmography - O.E.P. [BTS]) and at designing and interpreting experiments with patients. VA has participated in many international congresses all over the world as presenter and/or chairman in congresses' sessions as well as he has written many articles in scientific journals and books. 

List of publications 



\section{ORIGINAL RESEARCH ARTICLES AND REVIEWS}

Athanasopoulos D., Louvaris Z., Cherouveim E., Andrianopoulos V., Roussos Ch, Zakynthinos S., Vogiatzis I. Expiratory muscle loading increases intercostal muscle blood flow during leg exercise in healthy humans. Journal of Applied Physiology, 2010; 109:388-395.

Vogiatzis I., Andrianopoulos V., Louvaris Z., Cherouveim E., Spetsioti S., Vasilopoulou M., Athanasopoulos D. Quadriceps muscle blood flow and oxygen availability during repetitive bouts of isometric exercise in simulated sailing. Journal of Sports Science 2011; 29:1041-9.

Vogiatzis I., Louvaris Z., Habazettl H., Athanasopoulos D., Andrianopoulos V., Cherouveim E., Wagner H., Roussos C., Wagner PD, Zakynthinos S. Frontal cerebral cortex blood flow, oxygen delivery and oxygenation during normoxic and hypoxic exercise in athletes. Journal of Physiology 2011; 589:4027-39.

Louvaris Z., Zakynthinos SG, Aliverti A., Habazettl H., Vasilopoulou M., Andrianopoulos V., Wagner H., Wagner P.D., Vogiatzis I. Heliox increases quadriceps muscle oxygen delivery during exercise in COPD patients with and without dynamic hyperinflation. Journal of Applied Physiology, 2012; 113:1012-1023.

Vogiatzis I., Zakynthinos G., Andrianopoulos V. Mechanisms of physical activity limitation in Chronic Lung Diseases. Journal of Pulmonary Medicine 2012; 2012:634761.

Vogiatzis I., Louvaris Z., Habazettl H., Andrianopoulos V., Wagner H., Roussos Ch., Wagner P.D., Zakynthinos S. Cerebral cortex oxygen delivery and exercise limitation in patients with COPD. European Respiratory Journal 2013; 41:295-301.

Andrianopoulos V., Franssen F.M., Peeters J., Ubachs T., Bukari H., Groenen M., Burtin C., Vogiatzis I., Wouters EF., Spruit M.A. Exercise-induced oxygen desaturation in COPD patients without resting hypoxemia. Respiratory Physiology \& Neurobiology, 2013; 190C:40-46.

Andrianopoulos V., Louvaris Z., Orfanos I., Kouliaridou E., Atzemian Ch., Vogiatzis I. Quadriceps muscle oxygen availability between highly-ranked and club sailors during successive simulated hiking bouts. Journal of Athletic Enhancement, 2014; 3:2.

Andrianopoulos V., Klijn P., Franssen F.M., Spruit M.A. Exercise training in Pulmonary Rehabilitation. Clinics in Chest Medicine, 2014; 35:313-22. 
Andrianopoulos V., Wagers SS., Groenen M., Vanfleteren L., Franssen F.M., Smeenk W.J.M. F., Vogiatzis I., Wouters EF., Spruit M.A. Characteristics and determinants of endurance cycle ergometry and six-minute walk distance in patients with COPD. BMC: BioMed Central Pulmonary Medicine, 2014; 31:14:97.

Holland A.E., Spruit M.A., Troosters T., Puhan M.A., Pepin V., Saey D., McCormack MC., Carlin BW., Sciurba FC., Pitta F., Wanger J., Mclntyre N., Kaminsky D.A., Culver B.H., Revill S.M., Hernandes N.A., Andrianopoulos V., Camillo C.A., Mitchell K.E., Lee A.L., Hill C.J., Singh S.J. An official European Respiratory Society/American Thoracic Society technical standard: field walking tests in chronic respiratory disease. European Respiratory Journal, 2014; 44:1428-46.

Singh S.J., Puhan M.A., Andrianopoulos V., Hernandes N.A., Mitchell K.E., Hill C.J., Lee A.L., Camillo C.A., Troosters T., Spruit M.A., Carlin B.W., Wanger J., Pepin V., Saey D., Pitta F., Kaminsky D.A., McCormack M.C., McIntyre N., Culver B.H., Scuirba F.C., Revill S.M., Delafosse V., Holland A.E. An official systematic review of the European Respiratory Society/American Thoracic Society: measurement properties of field walking tests in chronic respiratory disease. European Respiratory Journal, 2014; 44:1447-78.

Vogiatzis I., Habazettl H., Louvaris Z., Andrianopoulos V., Wagner H., Zakynthinos S., Wagner P.D. A method for assessing heterogeneity of blood flow and metabolism in exercising normal human muscle by near-infrared spectroscopy. Journal of Applied Physiology (1985), 2015; 118:783-93.

Andrianopoulos V., Holland A., Singh S., Franssen F.M., Pennings H., Michels A., Vogiatzis I., Wouters E.F., Spruit M.A. Six-minute walk distance in patients with COPD: which reference equation should we use? Chronic Respiratory Disease Journal, 2015; 12:111-9.

Andrianopoulos V., Wouters E.F., Pinto-Plata V.M., Vanfleteren L.E., Bakke P.S., Franssen F.M., Agusti A., MacNee W., Rennard S.I., Tal-Singer R., Vogiatzis I., Vestbo J., Celli B.R., Spruit M.A. Prognostic value of variables derived from the six-minute walk test in patients with COPD: Results from the ECLIPSE study. Respiratory Medicine 2015; 109:1138-46.

Andrianopoulos V., Celli B.R., Franssen F.M., Pinto-Plata V.M., Calverley P.M., Vanfleteren L.E., Vogiatzis I., Vestbo J., Agusti A., Bakke P.S., Rennard S.I., MacNee W., Tal-Singer R., Yates J.C., Wouters E.F., Spruit M.A. Determinants of exercise-induced oxygen desaturation including pulmonary emphysema in COPD: Results from the ECLIPSE study. Respiratory Medicine 2016; 119:87-95. 
Andrianopoulos V., Vanfleteren L.E., Jarosch I., Gloeckl R., Schneeberger T. Wouters E.F., Spruit M.A., Kenn K. Transcutaneous carbon-dioxide partial pressure trends during sixminute walk test in patients with very severe COPD. Respiratory Physiology \& Neurobiology 2016; 233:52-59.

Gloeckl R., Jarosch I., Bengsch U., Claus M., Schneeberger T., Andrianopoulos V., Christle W.J., Hitzl W., Kenn K. What's the secret behind the benefits of whole-body vibration training in COPD? A randomized, controlled trial. Respiratory Medicine 2017; 126:17-24.

Andrianopoulos V., Gloeckl R., Vogiatzis I., Kenn K. Cognitive impairment in COPD: Should cognitive evaluation be part of respiratory assessment in patients with COPD? ERS Breathe 2017; 13:1-8.

\section{BOOK CHAPTERS}

Burtin Ch., Andrianopoulos V., Spruit M.A. Physical Activity and Physical Fitness in Health and Disease. In: Cardiorespiratory Physiotherapy: Adults and Paediatrics, 5th Edition, 2016. Elsevier publications.

Andrianopoulos V., Vogiatzis I. Windsurfing: The Physiology of Athletic Performance and Training. In: Extreme Sports Medicine, 2016. $1^{\text {st }}$ Ed. Springer publications. 
